\title{
Limit laws for the diameter of a set of random points from a distribution supported by a smoothly bounded set
}

\author{
Zur Erlangung des akademischen Grades eines \\ DOKTORS DER NATURWISSENSCHAFTEN \\ von der Fakultät für Mathematik des \\ Karlsruher Instituts für Technologie (KIT) \\ genehmigte \\ DISSERTATION
}

von

Dipl.-Math. oec. Michael Schrempp

aus Gengenbach

Tag der mündlichen Prüfung: 24. Mai 2017

Referent:

Prof. Dr. Norbert Henze

Korreferent:

Prof. Dr. Daniel Hug 



\section{ACKNOWLEDGMENTS}

First and foremost, I wholeheartedly thank my PhD advisor Prof. Dr. Norbert Henze for providing me with the opportunity to carry out my PhD studies over the past three years and his guidance during this time. Without the many discussions we had, the helpful remarks, reliance, encouragement and patience provided by him, completing this work would not have been possible. I also thank him for the chance of working as a scientific employee, enabling me to learn a lot by and about teaching mathematics to students. I also thank Prof. Dr. Daniel Hug for agreeing to act as referee on this thesis, for carefully reading the manuscript and for many helpful comments and suggestions.

Thanks are also due to all my current and former colleagues at the Institute of Stochastics at the Faculty of Mathematics at KIT. I always felt that the working atmosphere at "our" institute was truly pleasant.

Special thanks go out to the Talanx Foundation for generously granting me a scholarship for two semesters during my student days.

Finally, I thank my family, especially my parents Veronika and Albert Schrempp, for the loving and unconditional support during all the years. 

1 Introduction

2 Fundamentals 13

2.1 Notation . . . . . . . . . . . . . . . . . . . . . . . . . . . . . . 13

2.2 Point processes . . . . . . . . . . . . . . . . . . 14

3 Conditions, main results, and comments 17

3.1 Conditions . . . . . . . . . . . . . . . . . . . . . . . . . 17

3.2 Main results . . . . . . . . . . . . . . . . . . . . 26

3.3 Comments . . . . . . . . . . . . . . . . . . . . . . 30

4 Proof of Theorem $3.5 \quad 39$

4.1 Geometric considerations . . . . . . . . . . . . . . . . . . . . . 39

4.2 Convergence of Poisson random measures . . . . . . . . . . . . . . . . 45

4.3 Main part of the proof of Theorem 3.5 . . . . . . . . . . . . . . . . . 49

5 Generalizations 1 - Sets with unique diameter 55

5.1 More general densities supported by ellipsoids . . . . . . . . . . . 56

5.1 .1 General setting . . . . . . . . . . . . . . . 56 56

5.1 .2 Application to Pearson Type II distributions . . . . . . . . . . 70

5.1 .3 Technical details for Subsection 5.1.1 . . . . . . . . . . . . . 76

5.2 More general densities supported by general sets . . . . . . . . . . . . 82

5.2 .1 General considerations . . . . . . . . . . . . . . . 82

$5.2 .2 \quad$ A special class of densities on general sets . . . . . . . . . . . 84

5.2 .3 Technical details for Subsection 5.2.2 $\ldots \ldots \ldots$. . . . . . . . . 90

5.3 Joint convergence of the $k$ largest distances . . . . . . . . . . . . . . . 92

$5.4 \quad$ A different shape of $E$ close to the poles $\ldots \ldots$. . . . . . . . . . . 97 


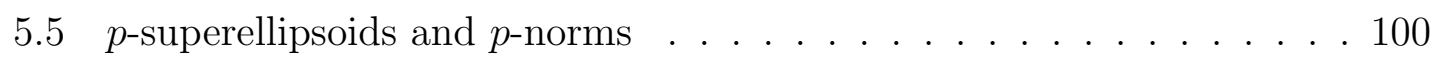

5.5 .1 Conditions and main results . . . . . . . . . . . . . . . 100

5.5.2 The behavior of $|x-y|_{p}$ close to the poles . . . . . . . . . 104

5.5.3 The shape of $E^{p}$ close to the poles . . . . . . . . . . . . . 109

5.5 .4 Main part of the proof of Theorem 5.15 . . . . . . . . . . . 110

5.6 No smoothness at the poles . . . . . . . . . . . . . . . 111

6 Generalizations 2 - Sets with no unique diameter 117

6.1 Several major axes . . . . . . . . . . . . . . . 117

6.1 .1 General setting . . . . . . . . . . . . . . . 117

$6.1 .2 \quad$ Application to $p$-balls for $p>2$. . . . . . . . . . . . . 122

6.2 Ellipsoids with no unique major half-axis . . . . . . . . . . . . . . . . 128

6.2 .1 Main results . . . . . . . . . . . . . . . . . . . 128

6.2 .2 Proofs of Lemma 6.8 and Lemma 6.9 . . . . . . . . . . . 136

\begin{tabular}{ll}
\hline Appendices & 141
\end{tabular}

\begin{tabular}{ll}
\hline A Principal curvatures and directions & 143
\end{tabular}

A.1 General theory . . . . . . . . . . . . . . . . . . . . . 143

A.1.1 The curvature of planar curves . . . . . . . . . . . . . . 143

A.1.2 The curvature of hypersurfaces . . . . . . . . . . . . . . . . 144

A.2 Calculation of principal curvatures . . . . . . . . . . . . . . . 147

A.2.1 Calculation for general hypersurfaces . . . . . . . . . . . . . 147

A.2.2 Calculation in our setting . . . . . . . . . . . . . . . 148

B Weak convergence of point processes 151

B.1 Weak convergence on metric spaces . . . . . . . . . . . . . . . . 151

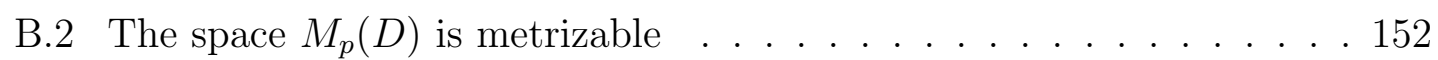

B.3 Results on weak convergence of point processes . . . . . . . . . 153

\begin{tabular}{ll}
\hline Bibliography & 157
\end{tabular} 


\section{CHAPTER 1}

\section{INTRODUCTION}

For some fixed integer $d \geq 2$, let $Z, Z_{1}, Z_{2}, \ldots$ be a sequence of independent and identically distributed (i.i.d.) $d$-dimensional random vectors, defined on a common probability space $(\Omega, \mathcal{A}, \mathbb{P})$. Throughout this thesis, we assume that the distribution $\mathbb{P}_{Z}$ of $Z$ is absolutely continuous with respect to Lebesgue measure. Writing $|\cdot|$ for the Euclidean norm on $\mathbb{R}^{d}$, the asymptotical behavior of the so-called maximum interpoint distance

$$
M_{n}:=\max _{1 \leq i, j \leq n}\left|Z_{i}-Z_{j}\right|
$$

as $n$ tends to infinity has been a topic of interest for more than 20 years. This behavior is closely related to the support $S \subset \mathbb{R}^{d}$ of $\mathbb{P}_{Z}$, which is the smallest closed set $C$ satisfying $\mathbb{P}_{Z}(C)=1$. Writing

$$
\operatorname{diam}(K):=\sup _{x, y \in K}|x-y|
$$

for the diameter of a set $K \subset \mathbb{R}^{d}$, we obviously have

$$
M_{n} \stackrel{\text { a.s. }}{\longrightarrow} \operatorname{diam}(S) \quad(\leq \infty)
$$

as $n \rightarrow \infty$. However, this result alone does not provide deep insight into the asymptotical behavior of $M_{n}$. For example, it is natural to ask for the speed of this convergence, depending on the distribution $\mathbb{P}_{Z}$. Being more precise, we are interested in finding two real-valued sequences $\left(a_{n}\right)_{n \in \mathbb{N}},\left(b_{n}\right)_{n \in \mathbb{N}}$ and a random variable $L$ with a non-degenerate 
distribution $\mathbb{P}_{L}$ over $\mathbb{R}$, so that $a_{n}\left(b_{n}-M_{n}\right)$ converges weakly to $L$ as $n \rightarrow \infty$. This means $\mathbb{P}\left(a_{n}\left(b_{n}-M_{n}\right) \leq t\right) \rightarrow \mathbb{P}(L \leq t)$ for each point of continuity $t$ of the distribution function of $L$ as $n \rightarrow \infty$, and we will briefly write $a_{n}\left(b_{n}-M_{n}\right) \stackrel{\mathcal{D}}{\longrightarrow} L$. Instead of investigating $a_{n}\left(b_{n}-M_{n}\right)$, some authors derived limiting results for $a_{n}\left(M_{n}-b_{n}\right)$. But, since we have $a_{n}\left(b_{n}-M_{n}\right)=-\left(a_{n}\left(M_{n}-b_{n}\right)\right)$, the continuous mapping theorem shows that $a_{n}\left(b_{n}-M_{n}\right) \stackrel{\mathcal{D}}{\longrightarrow} L$ implies $a_{n}\left(M_{n}-b_{n}\right) \stackrel{\mathcal{D}}{\longrightarrow}-L$, and vice versa. Hence, both approaches are equivalent in this setting.

If $S$ is bounded, i.e. if we have $\operatorname{diam}(S)<\infty$, we can choose $b_{n}=\operatorname{diam}(S)$ for each $n \in \mathbb{N}$. Then, the faster $M_{n}$ converges to its upper bound $\operatorname{diam}(S)$, the faster the sequence $\left(a_{n}\right)_{n \in \mathbb{N}}$ has to tend to infinity to obtain a non-degenerate limit distribution. If $S$ is unbounded, the sequence $\left(b_{n}\right)_{n \in \mathbb{N}}$ has to tend to infinity at 'the correct speed' and offers insight into the speed of the convergence of $M_{n}$ to infinity as $n \rightarrow \infty$.

For $d=1$ the asymptotical behavior of $M_{n}$ can be solved by using classical extreme value theory. In this case, $M_{n}$ is nothing but the sample range, i.e. we have

$$
M_{n}=\max _{1 \leq i \leq n} Z_{i}-\min _{1 \leq i \leq n} Z_{i}
$$

Since the random points $Z_{i}$ are independent, the largest and the smallest value of $Z_{1}, \ldots, Z_{n}$ are asymptotically independent. Hence, we can investigate the asymptotical behavior of $\max _{1 \leq i \leq n} Z_{i}$ and $\min _{1 \leq i \leq n} Z_{i}$ separately, and by convoluting the corresponding limit distributions we get the limit distribution of $M_{n}$. If $\mathbb{P}_{Z}$ is the uniform distribution on $[0,1]$, it can be shown that $n\left(1-M_{n}\right)$ converges weakly to the convolution of two independent exponential distributions with parameter 1, i.e. to a gamma distribution with shape parameter 2 and scale parameter 1 , see Lao [16, p. 2]. If $\mathbb{P}_{Z}$ is a symmetric distribution with a density function $f$ so that

$$
\frac{f(z)}{c|z|^{\alpha} e^{-\beta|z|^{\gamma}}} \rightarrow 1
$$

as $|z| \rightarrow \infty$ for some $c, \beta, \gamma>0$ and $\alpha \in \mathbb{R}$, Jammalamadaka and Janson [13] stated that

$$
\left(\beta^{\frac{1}{\gamma}} \gamma \log ^{1-\frac{1}{\gamma}} n\right) \cdot M_{n}-\left(2 \gamma \log n+\left(2 \frac{\alpha+1}{\gamma}-2\right) \log \log n+\log \left(\beta^{-\frac{2(\alpha+1)}{\gamma}} \gamma^{-2} c^{2}\right)\right)
$$

converges weakly to the convolution of two independent standard Gumbel-distributions, each of which has the distribution function $F(t)=\exp \left(-e^{-t}\right), t \in \mathbb{R}$. For example, this result covers the case that $\mathbb{P}_{Z}$ is the standard normal distribution. 
In the case $d \geq 2$ it is much more complicated to investigate the asymptotical behavior of $M_{n}$ since the representation (1.1) requires univariate observations. Results of classical extreme value theory can not be applied either, since, for instance, $\left|Z_{1}-Z_{2}\right|$ and $\left|Z_{1}-Z_{3}\right|$ are not independent. Results obtained so far mostly cover the case that the distribution $\mathbb{P}_{Z}$ is spherically symmetric, and they may roughly be classified according to whether $\mathbb{P}_{Z}$ has an unbounded or a bounded support. If $Z$ has a spherically symmetric normal distribution, Matthews and Rukhin [19] obtained a Gumbel limit distribution for $M_{n}$. Henze and Klein [10] generalized this result to the case that $Z$ has a spherically symmetric Kotz type distribution, i.e. a distribution with density

$$
f(z)=\frac{\kappa^{\frac{d}{2}+b-1} \Gamma\left(\frac{d}{2}\right)}{\pi^{\frac{d}{2}} \Gamma\left(\frac{d}{2}+b-1\right)}|z|^{2(b-1)} e^{-\kappa|z|^{2}}, \quad z \in \mathbb{R}^{d},
$$

where $2 b+d>2, \kappa>0$, and $\Gamma$ denotes the Gamma function. In this setting they stated the limit law

$$
\lim _{n \rightarrow \infty} \mathbb{P}\left(\sqrt{\frac{1}{\kappa} \log n}\left(M_{n}-2 \sqrt{\frac{1}{\kappa} \log n}-\frac{\frac{d+4 b-7}{2} \log _{2} n+\log _{3} n+a}{\sqrt{4 \kappa \log n}}\right) \leq \frac{t}{2 \kappa}\right)=e^{-e^{-t}},
$$

$t \in \mathbb{R}$, where

$$
a=\log \frac{(d-1) 2^{\frac{d-7}{2}} \Gamma\left(\frac{d}{2}\right)}{\sqrt{\pi} \Gamma^{2}\left(\frac{d}{2}+b-1\right)}
$$

and $\log _{2} n=\log \log n, \log _{3} n=\log \log _{2} n$. This result covers the case of a $d$ dimensional normal distribution for $b=1$ and $\kappa=1 / 2$. An even more general spherically symmetric setting has been studied by Jammalamadaka and Janson [13]. They considered spherically symmetric distributions $\mathbb{P}_{Z}$ with the property

$$
\mathbb{P}\left(|Z|>c_{n}+t d_{n}\right)=\frac{1+o(1)}{n} e^{-t}
$$

as $n \rightarrow \infty$, uniformly for all $t \in \mathbb{R}$ with $|t| \leq \frac{d-1}{2} \log \left(c_{n} / d_{n}\right)$, where $\left(c_{n}\right)_{n \in \mathbb{N}}$ and $\left(d_{n}\right)_{n \in \mathbb{N}}$ are sequences of positive numbers with $d_{n}=o\left(c_{n}\right)$. In this case,

$$
\lim _{n \rightarrow \infty} \mathbb{P}\left(\frac{M_{n}-2 c_{n}}{d_{n}}+\frac{d-1}{2} \log \frac{c_{n}}{d_{n}}-\log \log \frac{c_{n}}{d_{n}}-\log \frac{(d-1) 2^{d-4} \Gamma\left(\frac{d}{2}\right)}{\sqrt{\pi}} \leq t\right)=e^{-e^{-t}}
$$

holds true for each $t \in \mathbb{R}$. A sufficient condition for $(\overline{1.2})$ is that $\mathbb{P}_{Z}$ is spherically symmetric with a density $f$, where

$$
\frac{f(z)}{c|z|^{\alpha} e^{-\beta|z|^{\gamma}}} \rightarrow 1
$$


as $|z| \rightarrow \infty$ for some $c, \beta, \gamma>0$ and $\alpha \in \mathbb{R}$. Hence, [13] indeed covers the spherically symmetric Kotz type distributions, investigated by Henze and Klein [10. Henze and Lao [11] studied unbounded distributions $\mathbb{P}_{Z}$, for which the norm $|Z|$ and the directional part $Z /|Z|$ of $Z$ are independent and the right tail of the distribution of $|Z|$ decays like a power law. In this case, they showed a (non-Gumbel) limit distribution of $M_{n}$ that can be described in terms of a suitably defined Poisson point process. Finally, Demichel et al. [7] considered unbounded elliptical distributions of the form

$$
Z=T A W
$$

where $T$ is a positive and unbounded random variable, $A$ is an invertible $(d \times$ d)-dimensional matrix, and $W$ is uniformly distributed on the sphere $\mathcal{S}^{d-1}=$ $\left\{z \in \mathbb{R}^{d}:|z|=1\right\}$. In this case, the asymptotical behavior of $M_{n}$ depends on the right tail of the distribution function of $T$ and the multiplicity $k \in\{1, \ldots, d\}$ of the largest eigenvalue of $A$. In that work, it was assumed that $T$ lies in the max-domain of attraction of the Gumbel law. If the matrix $A$ has a single largest eigenvalue, [7] derives a limit law for $M_{n}$ that can be represented in terms of two independent Poisson point processes on $\mathbb{R}^{d}$. On the other hand, if $A$ has a multiple largest eigenvalue, and $T$ satisfies an additional technical assumption, they proved a Gumbel limit law for $M_{n}$. If $k=d$, the random vector $Z$ has a spherically symmetric distribution, and their result is the same as that stated by Jammalamadaka and Janson [13].

If $\mathbb{P}_{Z}$ has a bounded support, Appel et al. 3] obtained a convolution of two independent Weibull distributions as limit law of $M_{n}$ if $Z$ has a uniform distribution in a planar set with unique major axis and 'sub- $\sqrt{x}$ decay' of its boundary at the endpoints. Observe, that the latter property is not fulfilled if $\mathbb{P}_{Z}$ is supported by a proper ellipse $E$. In that case, Appel et al. [3] were only able to derive bounds for the limit law of $M_{n}$ if $Z$ has a uniform distribution. Being more precise, their result says

$$
\begin{aligned}
\mathbb{P}\left(W_{1}+W_{2} \leq \frac{t}{1+\gamma}\right) & \leq \liminf _{n \rightarrow \infty} \mathbb{P}\left(n^{\frac{2}{3}}\left(\operatorname{diam}(E)-M_{n}\right) \leq t\right) \\
& \leq \limsup _{n \rightarrow \infty} \mathbb{P}\left(n^{\frac{2}{3}}\left(\operatorname{diam}(E)-M_{n}\right) \leq t\right) \\
& \leq \mathbb{P}\left(W_{1}+W_{2} \leq t\right),
\end{aligned}
$$

where $t \geq 0$, and $\gamma>0$ is a constant, that depends solely on the two half-axes of $E$, and $W_{1}, W_{2}$ are two suitable i.i.d. Weibull random variables. Lao [16] and Mayer and Molchanov [20] deduced a Weibull limit distribution for $M_{n}$ in a very general setting if the distribution of $Z$ is supported by the $d$-dimensional unit ball $\mathbb{B}^{d}$ for 
$d \geq 2$. For instance, if $\mathbb{P}_{Z}$ is the uniform distribution in $\mathbb{B}^{d}$, they demonstrated that

$$
\lim _{n \rightarrow \infty} \mathbb{P}\left(\sigma^{\frac{2}{d+3}} \cdot n^{\frac{4}{d+3}} \cdot\left(2-M_{n}\right) \leq t\right)=1-\exp \left(-t^{\frac{d+3}{2}}\right), \quad t \geq 0,
$$

where

$$
\sigma=\frac{2^{d} d}{(d+1)(d+3) B\left(\frac{d}{2}+\frac{1}{2}, \frac{1}{2}\right)},
$$

and $B$ denotes the Beta function. Furthermore, Lao [16] obtained limit laws for $M_{n}$ if $\mathbb{P}_{Z}$ is uniform or non-uniform in the unit square, uniform in regular polygons, or uniform in the $d$-dimensional unit cube, $d \geq 2$. Moreover, if $\mathbb{P}_{Z}$ is uniform in a proper ellipse, Lao [16] improved the lower bound on the limit distribution of $M_{n}$ stated by Appel et al. [3], as given in (1.3). The exact limit behavior of $M_{n}$ if $\mathbb{P}_{Z}$ is uniform in an ellipse has been an open problem for many years. Without giving a proof, Jammalamadaka and Janson [13] stated that $n^{2 / 3}\left(2-M_{n}\right)$ has a limit distribution (involving two independent Poisson processes) if $Z$ has a uniform distribution in a proper ellipse with major axis of length 2. Schrempp [23] described this limit distribution in terms of two independent sequences of random variables, and in Schrempp [24] the result of Jammalamadaka and Janson [13] was generalized to the case that $\mathbb{P}_{Z}$ is uniform or non-uniform over a $d$-dimensional ellipsoid. Being more precise, the underlying set $E$ in [24] is

$$
E=\left\{z \in \mathbb{R}^{d}:\left(\frac{z_{1}}{a_{1}}\right)^{2}+\left(\frac{z_{2}}{a_{2}}\right)^{2}+\ldots+\left(\frac{z_{d}}{a_{d}}\right)^{2} \leq 1\right\}
$$

where $d \geq 2$ and $a_{1}>a_{2} \geq a_{3} \geq \ldots \geq a_{d}>0$. Since $a_{1}>a_{2}$, the ellipsoid $E$ has a unique major axis of length $2 a_{1}$ with 'poles' $\left(a_{1}, 0, \ldots, 0\right)$ and $\left(-a_{1}, 0, \ldots, 0\right)$. If the distribution $\mathbb{P}_{Z}$ is supported by such a set $E$ and $\mathbb{P}_{Z}(E \cap O)>0$ for each neighborhood $O$ of each of the two poles, the unique major axis makes sure that the asymptotical behavior of $M_{n}$ is determined solely by the shape of $\mathbb{P}_{Z}$ close to these poles. Schrempp [24] investigated distributions $\mathbb{P}_{Z}$ with a Lebesgue density $f$ on $E$, so that $f$ is continuous and bounded away from 0 near the poles. Hence, the uniform distribution on $E$ was a special case of that work. The assumptions stated in Schrempp [24] yield $M_{n} \rightarrow 2 a_{1}$ almost surely as $n$ tends to infinity. Furthermore, it turned out that $2 a_{1}-M_{n}$ has to be scaled by the factor $n^{2 /(d+1)}$ to obtain a non-degenerate limit distribution. In order to show this weak convergence, a related setting had been considered, in which the random points are the support of a specific series of Poisson point processes $\mathbf{Z}_{n}$ in $E$. Writing $\operatorname{diam}\left(\mathbf{Z}_{n}\right)$ for the diameter of the support of $\mathbf{Z}_{n}$, it turned out that $n^{2 /(d+1)}\left(2 a_{1}-\operatorname{diam}\left(\mathbf{Z}_{n}\right)\right)$ has a limiting distribution 
involving two independent Poisson processes that live on a subset $P$ of $\mathbb{R}^{d}$, the shape of which is determined by $a_{1}, \ldots, a_{d}$. By use of the so-called de-Poissonization technique it had been concluded that $n^{2 /(d+1)}\left(2 a_{1}-M_{n}\right)$ has the same limit distribution as $n$ tends to infinity.

Looking at the proofs given in Schrempp [24], it is quite obvious that only the values of the density at the poles and the curvature of the boundary $\partial E$ of $E$ at the poles determine the limiting distribution of $n^{2 /(d+1)}\left(2 a_{1}-M_{n}\right)$, but not the fact that $E$ is an ellipsoid. The latter observation was the starting point for this work: The main result of this work is a generalization of the result stated in Schrempp [24] to distributions that are supported by a $d$-dimensional set $E, d \geq 2$, with 'unique diameter' of length $2 a>0$ between the poles $(-a, 0, \ldots, 0)$ and $(a, 0, \ldots, 0)$ and a smooth boundary at the poles. The formal assumptions on the set $E$ are stated in Section 3.1. If the density $f$ of $Z$ on such a set $E$ is continuous and bounded away from 0 close to the poles, we can show that $n^{2 /(d+1)}\left(2 a-\operatorname{diam}\left(\mathbf{Z}_{n}\right)\right)$ has a non-degenerate limiting distribution also in this setting. This limit law again involves two independent Poisson processes that live on potentially different subsets $P_{\ell}$ and $P_{r}$ of $\mathbb{R}^{d}$. The shape of $P_{\ell}$ is only determined by the principal curvatures and the corresponding principal curvature directions of $\partial E$ at the left pole $(-a, 0, \ldots, 0)$. The same holds true for $P_{r}$ and the right pole $(a, 0, \ldots, 0)$.

In Chapter 2 we will fix our general notation and present a short introduction to point processes.

Chapter 3 contains our main result. After stating the assumptions on the underlying set $E$ and the distribution $\mathbb{P}_{Z}$ in Section 3.1, we formulate the main result, which is Theorem 3.5, in Section 3.2. This chapter closes in Section 3.3 with some comments on the intrinsic properties of sets that are covered by the assumptions stated in Section 3.1. The proof of Theorem 3.5 will be given in Chapter 4 .

Chapter 5 contains several generalizations of the main result. A common feature of these generalizations is that the underlying set $E$ has a 'unique diameter', attained by two points, the poles of $E$. In Section 5.1 and Section 5.2 we will consider more general distributions $\mathbb{P}_{Z}$, that include the so-called Pearson Type II distributions on $d$-dimensional ellipsoids. Section 5.3 establishes a limit theorem for the joint convergence of the $k$ largest distances among the random points $Z_{1}, \ldots, Z_{n}$, and in Section 5.4 we will discuss the case that the set $E$ has a slightly different shape close the poles. Moreover, Section 5.5 deals with $p$-norms and so-called ' $p$-superellipsoids', where $1 \leq p<\infty$, and Section 5.6 illustrates that the smoothness of the boundary of $E$ at the poles, as demanded in Section 3.1, is by no means necessary to prove 
results similar to that of Theorem 3.5.

Chapter 6 deals with generalizations of our main result to settings where $E$ does not have a 'unique diameter'. Sets with several but finitely many pairs of poles will be considered in Section 6.1, and Section 6.2 studies Pearson Type II distributions that are supported by an ellipsoid with at least two but less than $d$ major half-axes. In this setting, we can only show bounds for the limiting distribution, if such a limit law exists. To establish the asymptotical behavior in this case remains an open problem.

Finally, Appendix A presents some basics about the curvature of hypersurfaces, and in Appendix B we review several standard facts on the weak convergence of point processes. 



\section{CHAPTER 2}

\section{Fundamentals}

After fixing our general notation in Section 2.1, we present a short introduction to point processes in Section 2.2.

\subsection{Notation}

Vectors are understood as column vectors, but if there is no danger of misunderstanding, we write them - depending on the context - either as row or as column vectors.

Throughout this work, we use the abbreviation $\widetilde{z}:=\left(z_{2}, \ldots, z_{d}\right)$ for a point $z=\left(z_{1}, \ldots, z_{d}\right) \in \mathbb{R}^{d}$ to shorten the notation significantly. Given a function $s$ : $\mathbb{R}^{d-1} \rightarrow \mathbb{R}, \widetilde{z} \mapsto s(\widetilde{z})$, let $s_{j}(\widetilde{z})$ denote the partial derivative of $s$ with respect to the component $z_{j}$ for $j \in\{2, \ldots, d\}$. Notice that, for instance, $s_{2}$ stands for the partial derivative of $s$ with respect to $z_{2}$, not with respect to the second component of $\widetilde{z}$. The gradient $\left(s_{2}(\widetilde{z}), \ldots, s_{d}(\widetilde{z})\right)$ of $s$ at the point $\widetilde{z}$ will be denoted by $\nabla s(\widetilde{z})$. Likewise, we denote the second-order partial derivatives with respect to $z_{i}$ and $z_{j}$ by $s_{i j}(\widetilde{z})$, where $i, j \in\{2, \ldots, d\}$, and if the function $\mathbf{s}$ maps from $\mathbb{R}^{d-1}$ into $\mathbb{R}^{d}$, we write $\mathbf{s}_{i}$ and $\mathbf{s}_{i j}$ for the $d$-dimensional vectors of all first- and second-order partial derivatives with respect to $z_{i}$ and $z_{j}$.

Without stressing the dependence on the dimension, we write $\mathbf{0}$ for the origin in $\mathbb{R}^{i}$ and $\mathbf{e}_{j}$ for the $j$-th unit vector in $\mathbb{R}^{i}$ for $i, j \in \mathbb{N}:=\{1,2, \ldots\}$ with $j \leq i$. Whenever we do not emphasize the underlying dimension $i$, we mean $i=d$. The scalar product of two vectors $x, y \in \mathbb{R}^{i}$ will be denoted by $\langle x, y\rangle, i \in \mathbb{N}$. Let $B_{\varepsilon}(z)$ stand for 
the closed $i$-dimensional ball with center $z \in \mathbb{R}^{i}$ and radius $\varepsilon>0$ and $\mathbb{B}^{i}$ for the $i$-dimensional unit ball, $i \in \mathbb{N}$. Its volume will be denoted by $\omega_{i}$. For a subset $A \subset \mathbb{R}^{d}$ and $c>0$ we write $c \cdot A:=\{c \cdot z: z \in A\}$. In the sequel, $\mathcal{B}^{d}$ stands for the $\sigma$-field of Borel sets in $\mathbb{R}^{d}, \partial A$ for the boundary of a set $A \in \mathcal{B}^{d}$, $\operatorname{int}(A)$ for its interior, and we put $\mathbb{R}_{+}:=[0, \infty)$. Given a sequence $\left(A_{n}\right)_{n \geq 0}$ of Borel sets in $\mathbb{R}^{d}$, we write $A_{n} \uparrow A_{0}$ if $A_{i} \subset A_{i+1}$ for each $i \in \mathbb{N}$ and $\cup_{n \geq 1} A_{n}=A_{0}$. Without stressing the dependence on the dimension, $|\cdot|$ denotes the Euclidean norm on $\mathbb{R}^{i}$, and, whenever the dimension $i$ is clear, we write $\left\{\right.$ 'condition on $\left.z^{\prime}\right\}$ instead of $\left\{z \in \mathbb{R}^{i}\right.$ : 'condition on $z$ ' $\}, i \in \mathbb{N}$.

By $\Delta F$ we mean the Jacobian of a function $F: \mathbb{R}^{i} \rightarrow \mathbb{R}^{j}, i, j \in \mathbb{N}$, and the $i$-dimensional identity matrix will be denoted by $\mathrm{I}_{i}, i \in \mathbb{N}$. Given $a_{1}, \ldots, a_{i} \in \mathbb{R}$, we write $\operatorname{diag}\left(a_{1}, \ldots, a_{i}\right)$ for the corresponding diagonal matrix. In a similar way, if $A \in \mathbb{R}^{i \times i}$ and $B \in \mathbb{R}^{j \times j}$ are matrices, we write $\operatorname{diag}(A, B)$ for the corresponding $(i+j) \times(i+j)$-dimensional diagonal block matrix.

Each unspecified limit refers to $n \rightarrow \infty$, and for two real-valued sequences $\left(a_{n}\right)_{n \in \mathbb{N}}$ and $\left(b_{n}\right)_{n \in \mathbb{N}}$, where $b_{n} \neq 0$ for each $n \in \mathbb{N}$, we write $a_{n} \sim b_{n}$ if $a_{n} / b_{n} \rightarrow 1$.

In the sequel, $m_{d}$ stands for $d$-dimensional Lebesgue measure and $\mathcal{H}^{d}$ for $d$ dimensional Hausdorff measure. For a density $g$, a measure $\mu$ on $\mathbb{R}^{d}$ and a Borel set $A \in \mathcal{B}^{d}$ we put $\left.g\right|_{A}(z):=g(z)$ if $z \in A$ and 0 otherwise, and write $\left.\mu\right|_{A}(B):=\mu(A \cap B)$ if $B \in \mathcal{B}^{d}$.

Given a probability space $(\Omega, \mathcal{A}, \mathbb{P})$ and $A, B \in \mathcal{A}$, we use the notation $\mathbb{P}(A, B)$ for $\mathbb{P}(A \cap B)$. Convergence in distribution and equality in distribution will be denoted by $\stackrel{\mathcal{D}}{\longrightarrow}$ and $\stackrel{\mathcal{D}}{=}$, respectively. The components of a random vector $Z_{i}$ are given by $Z_{i}=\left(Z_{i, 1}, \ldots, Z_{i, d}\right)$ for $i \geq 1$. Finally, we write $N \stackrel{\mathcal{D}}{=} \operatorname{Po}(\lambda)$ if the random variable $N$ has a Poisson distribution with parameter $\lambda>0$.

\subsection{Point PRocesses}

With a few exceptions, we adopt the notation of Resnick [21], Chapter 3, for point processes. A point process on some space $D$, equipped with a $\sigma$-field $\mathcal{D}$, is a random distribution of points in $D$. A good way to formalize this description is to define point processes on $D$ as random measures $\chi$ on $D$ with $\chi(A) \in\{0,1, \ldots, \infty\}$ for each $A \in \mathcal{D}$. To this end, let $D$ be a subset of a compactified Euclidean space of finite dimension and $\mathcal{D}$ the Borel $\sigma$-field of subsets of $D$, i.e., the $\sigma$-field generated by the open sets. We write $\varepsilon_{z}$ for the Dirac measure centered at $z \in D$, and $M_{p}(D)$ denotes the set of all point measures $\chi$ of the form $\chi=\sum_{i=1}^{\infty} \varepsilon_{z_{i}}$, where $\left\{z_{i}, i \geq 1\right\}$ is a countable collection of not necessarily distinct points of $D$, that satisfies $\chi(K)<\infty$ for every compact set $K \in \mathcal{D}$. The latter property means that point measures are 
Radon measures. A point measure $\chi$ is called simple if $\chi(\{z\}) \in\{0,1\}$ for all $z \in D$. The set $M_{p}(D)$ of all point measures on $D$ is equipped with the smallest $\sigma$-field $\mathcal{M}_{p}(D)$ rendering the evaluation maps $\chi \mapsto \chi(A)$ from $M_{p}(D) \rightarrow[0, \infty]$ measurable for all $A \in \mathcal{D}$. Now we can define point processes as random elements of $M_{p}(D)$. Being more precise, a point process $\xi$ is a measurable map from some probability space $(\Omega, \mathcal{A}, \mathbb{P})$ into $\left(M_{p}(D), \mathcal{M}_{p}(D)\right)$. We call the point process $\xi$ simple if its distribution is concentrated on the simple point measures on $D$, i.e. if

$$
\mathbb{P}(\xi(\{z\}) \in\{0,1\} \text { for all } z \in D)=1 \text {. }
$$

Appendix B presents some basic facts about the weak convergence of point processes. In the following, we will mainly work with a very special class of point processes, the so-called Poisson processes. A Poisson process or Poisson random measure (PRM) with (Radon) intensity measure $\mu$ is a point process $\xi$ satisfying

$$
\mathbb{P}(\xi(A)=k)= \begin{cases}e^{-\mu(A) \frac{\mu(A)^{k}}{k !},} & \text { if } \mu(A)<\infty \\ 0, & \text { if } \mu(A)=\infty\end{cases}
$$

for $A \in \mathcal{D}$ and $k \in \mathbb{N} \cup\{0\}$. This property ensures $\xi(A)=\infty$ almost surely if $\mu(A)=\infty$ holds true. Moreover, $\xi\left(A_{1}\right), \ldots, \xi\left(A_{i}\right)$ are independent for any choice of $i \geq 2$ and mutually disjoint sets $A_{1}, \ldots, A_{i} \in \mathcal{D}$. We briefly write $\xi \stackrel{\mathcal{D}}{=} \operatorname{PRM}(\mu)$. If $\xi$ is a Poisson process with intensity measure $\mu,(2.1)$ means $\xi(A) \stackrel{\mathcal{D}}{=} \operatorname{Po}(\mu(A))$ and hence $\mathbb{E}[\xi(A)]=\mu(A)$ for $A \in \mathcal{D}$. According to Corollary 6.5 in Last and Penrose [17], there is for each Poisson process $\xi$ on $D$ a sequence $\mathcal{X}_{1}, \mathcal{X}_{2}, \ldots$ of random points in $D$ and a $\{0,1, \ldots, \infty\}$-valued random variable $N$ so that

$$
\xi=\sum_{i=1}^{N} \varepsilon_{\mathcal{X}_{i}}, \quad \text { almost surely. }
$$

Because of this property we use the notation $\xi=\left\{\mathcal{X}_{i}, i \geq 1\right\}$, whenever $\xi$ is a simple Poisson process and $\xi(D)=\infty$ almost surely. This terminology is motivated by the notion of a point process as a random set of points.

We will mainly use the bold letters $\mathbf{X}, \mathbf{Y}$ and $\mathbf{Z}$ to denote point processes, and the convention will be as follows: Point processes that are supported by the whole underlying set $E$ will get a name involving the letter $\mathbf{Z}$. In contrast, the letter $\mathbf{X}$ always stands for processes that live only on the left half $E \cap\left\{z_{1} \leq 0\right\}$ of $E$ and $\mathbf{Y}$ for those that are supported by the right half $E \cap\left\{z_{1} \geq 0\right\}$ of $E$. This distinction will be very 
useful to shorten the notation throughout this thesis. If, for instance, $\mathbf{X}=\left\{\mathcal{X}_{i}, i \geq 1\right\}$ is a point process on $\mathbb{R}^{d}$, we write $\mathcal{X}_{i}=\left(\mathcal{X}_{i, 1}, \ldots, \mathcal{X}_{i, d}\right)$ to denote the coordinates of $\mathcal{X}_{i}$.

Finally, we introduce a very special sequence of Poisson processes: In the following, the random variables $Z_{1}, Z_{2}, \ldots$ are i.i.d. with common distribution $\mathbb{P}_{Z}$, and let $N_{n}$ be independent of this sequence and have a Poisson distribution with parameter $n \in \mathbb{N}$. Defining the point processes

$$
\mathbf{Z}_{n}:=\sum_{i=1}^{N_{n}} \varepsilon_{Z_{i}}, \quad n \in \mathbb{N}
$$

we get

$$
\operatorname{diam}\left(\mathbf{Z}_{n}\right)=M_{N_{n}}=\max _{1 \leq i, j \leq N_{n}}\left|Z_{i}-Z_{j}\right|,
$$

and $\mathbf{Z}_{n}$ is a Poisson process in $\mathbb{R}^{d}$ with intensity measure $n \mathbb{P}_{Z}$. Observe that the expected number of points of $\mathbf{Z}_{n}$ is exactly $n$ for each $n \in \mathbb{N}$. In order to prove limiting results for $M_{n}$, it will be very useful to consider these processes. 


\section{CHAPTER 3}

\section{Conditions, MAIn RESUlts, AND COMMENTS}

This chapter, which contains our main results, is divided into three sections. Besides our assumptions on the underlying set $E$, Section 3.1 contains some important implications of those conditions and definitions that are necessary for stating our main results, which are given in Section 3.2. Section 3.3 takes a closer look at some significant properties of sets that are covered by the assumptions given in Section 3.1.

\subsection{Conditions}

Our basic assumption on the shape and the orientation of the underlying set $E$ is that its finite diameter is attained by exactly one pair of points, both of which lie on the $z_{1}$-axis. Being more precise, we assume the following:

Condition 1. Let $E \subset \mathbb{R}^{d}$ be a closed subset with $0<2 a=\operatorname{diam}(E)<\infty$ and $(-a, \mathbf{0}),(a, \mathbf{0}) \in E$. Furthermore, we assume

$$
|x-y|<2 a \quad \text { for each } \quad(x, y) \in(E \backslash\{(-a, 0),(a, 0)\}) \times E .
$$

Speaking of a 'unique diameter between the points $(-a, \mathbf{0})$ and $(a, \mathbf{0})$ ' or simply of a 'unique diameter', we will always mean that the underlying set satisfies Condition 1. The two points $(-a, \mathbf{0}),(a, \mathbf{0}) \in E$ are henceforth called the 'poles' of $E$. There is no loss of generality in assuming that the poles of $E$ are given by $(-a, \mathbf{0})$ and $(a, \mathbf{0})$. For every set having a diameter of length $2 a>0$ we can find a suitable coordinate system so that this assumption is satisfied. Since we will consider distributions 
with $m_{d}$-densities supported by $E$, it will be no loss of generality either that we assume $E$ to be closed. Notice, that this assumption in Condition 1 is very important. Otherwise, condition (3.1) would not be sufficient for our purposes, as the following example illustrates: Let $d=2$ and $E^{\prime}$ be the convex hull of $\{(-1,0),(1,0),(0, \sqrt{3})\}$. Notice that $\partial E^{\prime}$ is an equilateral triangle, and put

$$
E^{\prime \prime}:=\left(E^{\prime} \cap\left\{z_{2} \leq 1\right\}\right) \cup \operatorname{int}\left(E^{\prime} \cap\left\{z_{2}>1\right\}\right)
$$

See Figure 3.1 for an illustration of this set. This non-closed set fulfills both

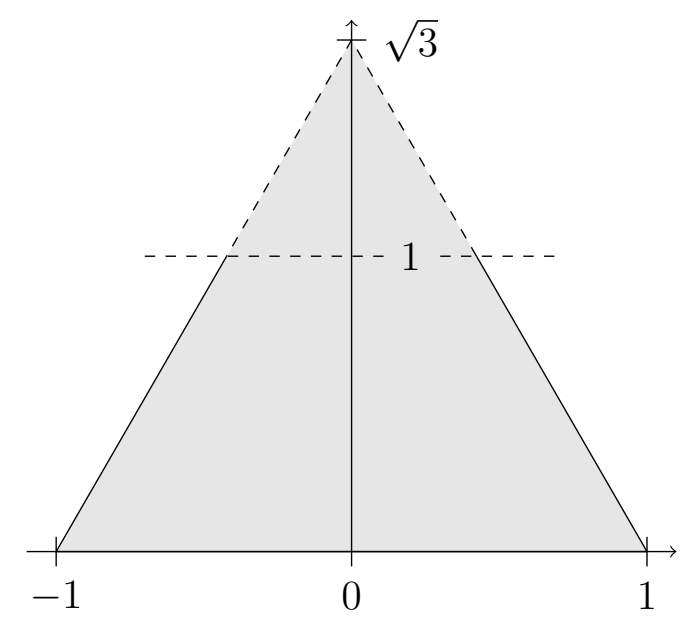

Figure 3.1: The set $E^{\prime \prime}$

$\operatorname{diam}\left(E^{\prime \prime}\right)=2 a$ and condition $(\sqrt[3.1]{)}$ with $a=1$. But if we consider - for example the uniform distribution in $E^{\prime \prime}$, we would get very complicated dependencies between large distances: In this setting, a random point lying close to the vertex $(-1,0)$ can determine the maximum interpoint distance either with a point lying close to the vertex $(1,0)$ or with a point lying close to the vertex $(0, \sqrt{3})$. By assuming $E$ to be closed, condition (3.1) guarantees that $M_{n}$ will be determined by two points lying close to $(-a, \mathbf{0})$ and $(a, \mathbf{0})$, respectively, at least for large $n$ and a suitable distribution $\mathbb{P}_{Z}$.

Our assumption on the shape of $E$ close to both poles is as follows: 
Condition 2. There are constants $\delta_{\ell}, \delta_{r} \in(0, a]$, open neighborhoods $O_{\ell}, O_{r} \subset \mathbb{R}^{d-1}$ of $\mathbf{0} \in \mathbb{R}^{d-1}$ and twice continuously differentiable functions $s^{\ell}: O_{\ell} \rightarrow \mathbb{R}_{+}, s^{r}: O_{r} \rightarrow$ $\mathbb{R}_{+}$, so that

$$
\begin{aligned}
E_{\ell} & :=E \cap\left\{z_{1}<-a+\delta_{\ell}\right\} \\
& =\left\{\left(z_{1}, \widetilde{z}\right) \in \mathbb{R}^{d}:-a+s^{\ell}(\widetilde{z}) \leq z_{1}<-a+\delta_{\ell}, \widetilde{z} \in O_{\ell}\right\}
\end{aligned}
$$

and

$$
\begin{aligned}
E_{r} & :=E \cap\left\{a-\delta_{r}<z_{1}\right\} \\
& =\left\{\left(z_{1}, \widetilde{z}\right) \in \mathbb{R}^{d}: a-\delta_{r}<z_{1} \leq a-s^{r}(\widetilde{z}), \widetilde{z} \in O_{r}\right\} .
\end{aligned}
$$

The notation of partial derivatives by subscripts throughout this work requires the usage of superscripts to distinguish the functions $s^{\ell}$ and $s^{r}$. Since $(-a, \mathbf{0}),(a, \mathbf{0}) \in E$, we have $s^{\ell}(\mathbf{0})=s^{r}(\mathbf{0})=0$. The 'outer boundaries' of $E_{\ell}$ and $E_{r}$ will be denoted by

$$
M_{\ell}:=\left\{\left(z_{1}, \widetilde{z}\right) \in \mathbb{R}^{d}: z_{1}=-a+s^{\ell}(\widetilde{z}), \widetilde{z} \in O_{\ell}\right\}
$$

and

$$
M_{r}:=\left\{\left(z_{1}, \widetilde{z}\right) \in \mathbb{R}^{d}: z_{1}=a-s^{r}(\widetilde{z}), \widetilde{z} \in O_{r}\right\}
$$

respectively. Figure 3.2 displays the initial situation given by Condition 1 and Condition 2. Notice that $E$ can be defined in any way on the set

$$
\left\{z \in \mathbb{R}^{d}:-a+\delta_{\ell} \leq z_{1} \leq a-\delta_{r}\right\}
$$

as long as Condition 1 is satisfied.

It will be very convenient to consider the boundaries $M_{\ell}$ and $M_{r}$ as images of two appropriately defined hypersurfaces. For this purpose, we put

$$
\mathbf{s}^{\ell}: O_{\ell} \rightarrow \mathbb{R}^{d}, \mathbf{s}^{\ell}(\widetilde{z}):=\left(-a+s^{\ell}(\widetilde{z}), \widetilde{z}\right)
$$

and

$$
\mathbf{s}^{r}: O_{r} \rightarrow \mathbb{R}^{d}, \mathbf{s}^{r}(\widetilde{z}):=\left(a-s^{r}(\widetilde{z}), \widetilde{z}\right) .
$$




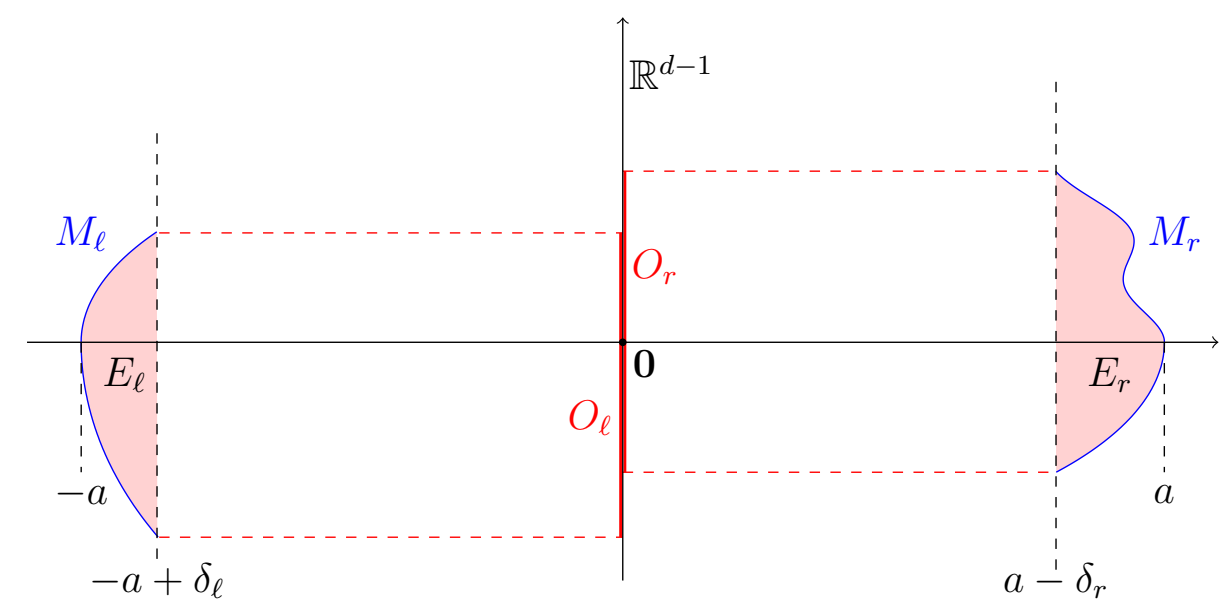

Figure 3.2: The initial situation given by Condition 1 and Condition 2.

Formally, the inverse image of a hypersurface in $\mathbb{R}^{d}$ has to be an open subset of $\mathbb{R}^{d-1}$. Due to this convention, we have demanded $O_{\ell}$ and $O_{r}$ to be open. This requirement corresponds to the intersection of $E$ with $\left\{z_{1}<-a+\delta_{\ell}\right\}$ instead of $\left\{z_{1} \leq-a+\delta_{\ell}\right\}$ in (3.2), and with $\left\{z_{1}>a-\delta_{r}\right\}$ instead of $\left\{z_{1} \geq a-\delta_{r}\right\}$ in (3.3). Since we will have to investigate $E$ close to the poles, this convention will be no problem for our purposes: For instance, the set $E \cap\left\{z_{1}=-a+\delta_{\ell}\right\}$ will be completely irrelevant for the limiting behavior of $M_{n}$.

Remark 3.1. A common way to define a hypersurface as the graph of a function $s: O \rightarrow \mathbb{R}$ with $O \subset \mathbb{R}^{d-1}$ is to describe the last component $z_{d}$ via $z_{1}, \ldots, z_{d-1}$, i.e. $\mathbf{s}\left(z_{1}, \ldots, z_{d-1}\right):=\left(z_{1}, \ldots, z_{d-1}, s\left(z_{1}, \ldots, z_{d-1}\right)\right)$. We deliberately deviate from this convention for two reasons: The orientation of $E$ given by Condition 1 and Condition 2 is the same as in Appel et al. [3] and Schrempp [24], and it conveniently emphasizes the very special role of the first component in our main theorem.

Since $s^{\ell}$ and $s^{r}$ are twice continuously differentiable, the second-order Taylor series expansions of these functions are defined. Writing $H_{i}$ for the Hessian of $s^{i}$ at the point $\mathbf{0}$, we get

$$
s^{i}(\widetilde{z})=0+\nabla s^{i}(\mathbf{0})^{\top} \widetilde{z}+\frac{1}{2} \widetilde{z}^{\top} H_{i} \widetilde{z}+R_{i}(\widetilde{z}),
$$

where $R_{i}(\widetilde{z})=o\left(|\widetilde{z}|^{2}\right)$ and $i \in\{\ell, r\}$. In view of the unique diameter of $E$ between $(-a, \mathbf{0})$ and $(a, \mathbf{0})$, we know the following facts about $\nabla s^{i}(\mathbf{0})$ and $H_{i}, i \in\{\ell, r\}$ :

Lemma 3.2. For $i \in\{\ell, r\}$ we have $\nabla s^{i}(\mathbf{0})=\mathbf{0}$. Furthermore, the matrix $H_{i}$ is symmetric and positive definite, and all $d-1$ eigenvalues of $H_{i}$ are larger than $1 / 2 a$.

Proof. We only consider $i=\ell$. It is clear that $H_{\ell}$ is symmetric, since $s^{\ell}$ is a twice 
continuously differentiable function. From Condition 1] we know that

$$
E \subset B_{2 a}((a, \mathbf{0})) \quad \text { and } \quad E \cap \partial B_{2 a}((a, \mathbf{0}))=\{(-a, \mathbf{0})\}
$$

Writing $O_{t}:=\left\{\widetilde{z} \in \mathbb{R}^{d-1}:|\widetilde{z}|<2 a\right\}$ and defining the mapping $t: O_{t} \rightarrow \mathbb{R}, \widetilde{z} \mapsto a-$ $\sqrt{4 a^{2}-z_{2}^{2}-\ldots-z_{d}^{2}}$, the boundary of $B_{2 a}((a, \mathbf{0}))$ in $\left\{z_{1}<a\right\}$ can be parameterized as a hypersurface via

$$
\mathbf{t}:\left\{\begin{array}{l}
O_{t} \rightarrow \mathbb{R}^{d}, \\
\widetilde{z} \mapsto(t(\widetilde{z}), \widetilde{z}) .
\end{array}\right.
$$

For $j, k \in\{2, \ldots, d\}$, we obtain

$$
\begin{aligned}
t_{j}(\widetilde{z}) & =\left(4 a^{2}-z_{2}^{2}-\ldots-z_{d}^{2}\right)^{-\frac{1}{2}} \cdot z_{j} \\
t_{j k}(\widetilde{z}) & =\left(4 a^{2}-z_{2}^{2}-\ldots-z_{d}^{2}\right)^{-\frac{3}{2}} \cdot z_{j} z_{k}+\left(4 a^{2}-z_{2}^{2}-\ldots-z_{d}^{2}\right)^{-\frac{1}{2}} \cdot \delta_{j k}
\end{aligned}
$$

Hence, $\nabla t(\mathbf{0})=\mathbf{0}$, and the Hessian of $t$ at $\mathbf{0}$ is given by $H_{t}:=\frac{1}{2 a} \mathrm{I}_{d-1}$. So, the second-order Taylor series expansion of $t$ at this point has the form

$$
t(\widetilde{z})=-a+\mathbf{0}^{\top} \widetilde{z}+\frac{1}{2} \widetilde{z}^{\top} H_{t} \widetilde{z}+R_{t}(\widetilde{z})
$$

where $R_{t}(\widetilde{z})=o\left(|\widetilde{z}|^{2}\right)$. In view of $\left(\overline{3.5)}\right.$ and Condition 2, we have $t(\widetilde{z})<-a+s^{\ell}(\widetilde{z})$ for each $\widetilde{z} \in O_{\ell} \backslash\{\mathbf{0}\}$ (observe that $(3.5)$ ensures $O_{\ell} \subset O_{t}$ ). Using (3.4) and (3.6), this inequality can be rewritten as

$$
-a+\mathbf{0}^{\top} \widetilde{z}+\frac{1}{2} \widetilde{z}^{\top} H_{t} \widetilde{z}+R_{t}(\widetilde{z})<-a+\nabla s^{\ell}(\mathbf{0})^{\top} \widetilde{z}+\frac{1}{2} \widetilde{z}^{\top} H_{\ell} \widetilde{z}+R_{\ell}(\widetilde{z}),
$$

and hence

$$
0<\nabla s^{\ell}(\mathbf{0})^{\top} \widetilde{z}+\frac{1}{2} \widetilde{z}^{\top}\left(H_{\ell}-H_{t}\right) \widetilde{z}+\left(R_{\ell}(\widetilde{z})-R_{t}(\widetilde{z})\right)
$$

for each $\widetilde{z} \in O_{\ell} \backslash\{\mathbf{0}\}$. Since $R_{\ell}(\widetilde{z})-R_{t}(\widetilde{z})=o\left(|\widetilde{z}|^{2}\right)$, this inequality shows $\nabla s^{\ell}(\mathbf{0})=\mathbf{0}$ and that the matrix $H_{\ell}-H_{t}$ is positive definite. Remembering $H_{t}=\frac{1}{2 a} \mathrm{I}_{d-1}, H_{\ell}$ has to be positive definite, too, and all eigenvalues of $H_{\ell}$ have to be larger than $1 / 2 a$.

Remark 3.3. For $i \in\{\ell, r\}$ the first partial derivatives of $\mathbf{s}^{i}$ are given by

$$
\mathbf{s}_{2}^{i}(\widetilde{z})=\left(s_{2}^{i}(\widetilde{z}), 1,0, \ldots, 0\right), \ldots, \mathbf{s}_{d}^{i}(\widetilde{z})=\left(s_{d}^{i}(\widetilde{z}), 0, \ldots, 0,1\right) .
$$

These $d-1$ vectors are linearly independent for each $\widetilde{z} \in O_{i}$, which means that the hypersurfaces $\mathbf{s}^{\ell}$ and $\mathbf{s}^{r}$ are regular, see Definition 3.1.2 in Csikós [5]. From 
Lemma 3.2 we further know $\mathbf{s}_{j}^{i}(\mathbf{0})=\mathbf{e}_{j}$ for $i \in\{\ell, r\}$ and each $j \in\{2, \ldots, d\}$. Hence, the two unit normal vectors of the hypersurface $\mathbf{s}^{i}$ at the pole $\mathbf{s}^{i}(\mathbf{0})$ are given by $\pm \mathbf{e}_{1}$.

Putting (3.4) and Lemma 3.2 together, it is clear that the second-order Taylor series expansions of $s^{i}$ at the point $\mathbf{0}$ is

$$
s^{i}(\widetilde{z})=\frac{1}{2} \widetilde{z}^{\top} H_{i} \widetilde{z}+R_{i}(\widetilde{z})
$$

where $R_{i}(\widetilde{z})=o\left(|\widetilde{z}|^{2}\right)$ and $i \in\{\ell, r\}$. From $(3.2)$ and $(3.3)$ we obtain the representations

$$
E_{\ell}=\left\{\left(z_{1}, \widetilde{z}\right) \in \mathbb{R}^{d}:-a+\frac{1}{2} \widetilde{z}^{\top} H_{\ell} \widetilde{z}+R_{\ell}(\widetilde{z}) \leq z_{1}<-a+\delta_{\ell}, \widetilde{z} \in O_{\ell}\right\}
$$

and

$$
E_{r}=\left\{\left(z_{1}, \widetilde{z}\right) \in \mathbb{R}^{d}: a-\delta_{r}<z_{1} \leq a-\frac{1}{2} \widetilde{z}^{\top} H_{r} \widetilde{z}-R_{r}(\widetilde{z}), \widetilde{z} \in O_{r}\right\},
$$

which will be widely used throughout this work.

According to Lemma 3.2, the matrices $H_{\ell}$ and $H_{r}$ are orthogonally diagonalizable and all eigenvalues, denoted by $\frac{1}{2 a}<\kappa_{2}^{i} \leq \ldots \leq \kappa_{d}^{i}, i \in\{\ell, r\}$, in ascending order, are real-valued and positive. The subscripts $2, \ldots, d$ instead of $1, \ldots, d-1$ are chosen deliberately. Because of the very close connection between these eigenvalues and the components $z_{2}, \ldots, z_{d}$ in our main theorem, this notation is much more intuitive for our purposes. Observe especially $(3.15)$ on page 30 for the aforementioned connection. For $i \in\{\ell, r\}$ we choose an orthonormal basis $\left\{\mathbf{u}_{2}^{i}, \ldots, \mathbf{u}_{d}^{i}\right\}$ of $\mathbb{R}^{d-1}$, consisting of corresponding eigenvectors; namely $H_{i} \mathbf{u}_{j}^{i}=\kappa_{j}^{i} \mathbf{u}_{j}^{i}$ for $j \in\{2, \ldots, d\}$. Putting $U_{i}:=\left(\mathbf{u}_{2}^{i}|\ldots| \mathbf{u}_{d}^{i}\right)$, we have $U_{i} U_{i}^{\top}=\mathrm{I}_{d-1}$ and $U_{i}^{\top} H_{i} U_{i}=\operatorname{diag}\left(\kappa_{2}^{i}, \ldots, \kappa_{d}^{i}\right)=: D_{i}$.

Looking at Subsection A.2.2 - especially its ending - we know (because of $\nabla s^{i}(\mathbf{0})=$ 0) that the eigenvalues $\kappa_{j}^{i}$ of the Hessian $H_{i}$ are exactly the principal curvatures of the hypersurface $\mathbf{s}^{i}$ at the pole $\mathbf{s}^{i}(\mathbf{0})$ with respect to the unit normal vector

$$
\mathbf{N}^{i}(\mathbf{0}):=\left\{\begin{aligned}
\mathbf{e}_{1}, & i=\ell \\
-\mathbf{e}_{1}, & i=r
\end{aligned}\right.
$$


We can further conclude that

$$
\mathbf{v}_{j}^{i}:=\left(\begin{array}{c}
0 \\
\mathbf{u}_{j}^{i}
\end{array}\right) \in \mathbb{R}^{d}
$$

are the corresponding principal curvature directions.

It is quite obvious that Condition 1 restricts the possible principal curvatures and the corresponding principal curvature directions of $\mathbf{s}^{\ell}$ and $\mathbf{s}^{r}$ at the poles. It would be desirable to find a one-to-one relation between the unique diameter of $E$ assumed in Condition 1 on the one hand and all possible principal curvatures and directions of the hypersurfaces $\mathbf{s}^{\ell}$ and $\mathbf{s}^{r}$ at the poles on the other hand. But describing this relation in its whole generality would be technically very involved. Fortunately, we can state a simple but still very general condition on the principal curvatures and directions of $\partial E$ at the poles to guarantee that $E \cap\left\{\left|z_{1}\right|>a-\delta\right\}$ has a unique diameter between $(-a, \mathbf{0})$ and $(a, \mathbf{0})$ for $\delta>0$ sufficiently small. Unless otherwise stated we will always study sets fulfilling the following condition:

Condition 3. For some constant $\eta \in(0,1)$, the $2(d-1) \times 2(d-1)$-dimensional matrix

$$
A(\eta):=\left(\begin{array}{cc}
2 a \eta D_{\ell}-\mathrm{I}_{d-1} & U_{\ell}^{\top} U_{r} \\
U_{r}^{\top} U_{\ell} & 2 a \eta D_{r}-\mathrm{I}_{d-1}
\end{array}\right)
$$

is positive semi-definite.

We will briefly write $A(\eta) \geq 0$ to denote this property. Notice that $A\left(\eta_{1}\right) \geq 0$ implies $A\left(\eta_{2}\right) \geq 0$ for each $\eta_{2}>\eta_{1}$ since $D_{\ell}$ and $D_{r}$ are diagonal matrices with positive entries on their main diagonals. Due to the fact that $D_{\ell}, D_{r}, U_{\ell}$ and $U_{r}$ depend only on the curvature of $\partial E$ at the poles, Condition 3 is obviously not sufficient to ensure (3.1) (figuring in Condition 1) for the whole set $E$. But Lemma 3.9 will show that Condition 3 guarantees that (3.1) holds true for $E$ replaced with $E \cap\left\{\left|z_{1}\right|>a-\delta\right\}$ and $\delta>0$ sufficiently small. This assertion can be interpreted as Condition 3 ensures the unique diameter of $E$ close to the poles'. Focussing on sets satisfying Condition 3 will be no strong limitation in the following sense: If $A(1)$ is not positive semi-definite, then $E$ cannot have a unique diameter between the poles, see Lemma 3.11. Hence, the only relevant case not covered by Condition 3 is given by

$$
A(1) \geq 0, \quad \text { but } \quad A(\eta) \nsupseteq 0 \quad \text { for each } \quad \eta \in(0,1) .
$$


In this case one would have to check the relation between the two error functions $R_{\ell}$ and $R_{r}$ for all possible combinations of two directions in $\mathbb{R}^{d-1}$, see the following very simple example for an illustration:

Example 3.4. For $d=2$, a set not covered by Condition 3 is

$$
E_{1}:=B_{a}(\mathbf{0})
$$

for some $a>0$. It is a well-known fact that a circle with radius $a>0$ has constant curvature $1 / a$, i.e. $\kappa_{2}^{\ell}=\kappa_{2}^{r}=1 / a$. A proof of this result will be given indirectly by the much more general calculations in the proof of Lemma 6.4, see especially Remark 6.5. So, we have $H_{\ell}=H_{r}=D_{\ell}=D_{r}=\left(\frac{1}{a}\right)$ and

$$
A(\eta)=\left(\begin{array}{cc}
2 \eta-1 & 1 \\
1 & 2 \eta-1
\end{array}\right)
$$

The smallest $\eta>0$ with $A(\eta) \geq 0$ is given by $\eta=1$. For $a=1$, putting $h\left(z_{2}\right):=$ $\sqrt{1-z_{2}^{2}}$, we have

$$
E_{1}=\left\{z \in \mathbb{R}^{2}:-h\left(z_{2}\right) \leq z_{1} \leq h\left(z_{2}\right),\left|z_{2}\right| \leq 1\right\}
$$

Now we manipulate this unit-ball via

$$
E_{2}:=\left\{z \in \mathbb{R}^{2}:-h\left(z_{2}\right)+z_{2}^{4} \leq z_{1} \leq h\left(z_{2}\right)-z_{2}^{4},\left|z_{2}\right| \leq \frac{3}{4}\right\}
$$

and

$$
E_{3}:=\left\{z \in \mathbb{R}^{2}:-h\left(z_{2}\right)-\frac{3}{10} z_{2}^{4} \leq z_{1} \leq h\left(z_{2}\right)+\frac{3}{10} z_{2}^{4},\left|z_{2}\right| \leq 1\right\}
$$

Figure 3.3 displays the boundaries of the sets $E_{1}, E_{2}$ and $E_{3}$. Although all three sets have the same principal curvature 1 at the points $(-1,0)$ and $(1,0)$, we observe three completely different situations as to the uniqueness of the diameter between these points. While $E_{2}$ has a unique diameter between the points $(-1,0)$ and $(1,0)$, the diameter of the ball $E_{1}$ is not unique, and for $E_{3}$ we even have $|(-1,0)-(1,0)|<$ $\operatorname{diam}\left(E_{3}\right)$. So, Condition 1 is only fulfilled for the set $E_{2}$. This example illustrates on the one hand that Condition 3 is only sufficient for showing the unique diameter of $E \cap\left\{\left|z_{1}\right|>a-\delta\right\}$ for small $\delta>0$, but not necessary. On the other hand, we can 


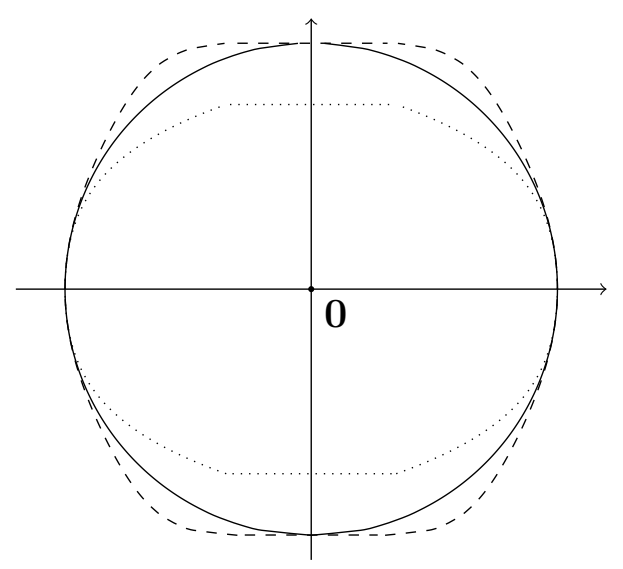

Figure 3.3: The boundaries of the sets $E_{1}$ (solid), $E_{2}$ (dotted) and $E_{3}$ (dashed) in Example 3.4.

see that the situation

$$
A(1) \geq 0, \quad \text { but } \quad A(\eta) \nsupseteq 0 \quad \text { for each } \quad \eta \in(0,1),
$$

not covered by Condition 3, can be very intricate to handle. For checking Condition 1 (close to the poles) in higher dimensions, one would have to examine the relation between the two error functions $R_{\ell}$ and $R_{r}$ figuring in (3.7) and (3.8) with respect to all possible combinations of two directions in $\mathbb{R}^{d-1}$.

At first sight, Condition 3 looks quite technical. A much more intuitive and sufficient, but not necessary condition for Condition 3 to hold is

$$
\frac{1}{\kappa_{2}^{\ell}}+\frac{1}{\kappa_{2}^{r}}<2 a
$$

see Lemma 3.12. We may thus check Condition 1 (at least close to the poles) for many sets by merely looking at the smallest principal curvatures at the poles.

Now that we have stated our conditions on the underlying set $E$, we can focus on distributions supported by $E$. In this section we consider distributions $\mathbb{P}_{Z}$ with a Lebesgue density $f$ on $E$ satisfying the following property of continuity at the poles:

Condition 4. Let $f: E \rightarrow \mathbb{R}_{+}$with $\int_{E} f(z) \mathrm{d} z=1$. We further assume that $f$ is continuous at the poles $(-a, \mathbf{0}),(a, \mathbf{0})$ with

$$
p_{\ell}:=f(-a, \mathbf{0})>0 \quad \text { and } \quad p_{r}:=f(a, \mathbf{0})>0 .
$$


Defining the 'pole-caps of length $\delta$ ' via

$$
E_{\ell, \delta}:=E_{\ell} \cap\left\{-a \leq z_{1} \leq-a+\delta\right\} \quad \text { and } \quad E_{r, \delta}:=E_{r} \cap\left\{a-\delta \leq z_{1} \leq a\right\}
$$

for $0<\delta<\min \left\{\delta_{\ell}, \delta_{r}\right\}$, the property of continuity assumed in Condition 4 can be rewritten as $f(z)=p_{i}(1+o(1))$, where $o(1)$ is uniformly on $E_{i, \delta}$ as $\delta \rightarrow 0, i \in\{\ell, r\}$. In the proofs to follow, we only use this characterization. Now, we only need one more definition before we can formulate our main result. Putting

$$
P(H):=\left\{\left(z_{1}, \widetilde{z}\right) \in \mathbb{R}^{d}: \frac{1}{2} \widetilde{z}^{\top} H \widetilde{z} \leq z_{1}\right\}
$$

for some $(d-1) \times(d-1)$-dimensional matrix $H$, the set $P\left(H_{\ell}\right)$ (resp. $\left.P\left(H_{r}\right)\right)$ describes the shape of $E$ near the left (resp. right) pole if we 'look through a suitably distorted magnifying glass', see Lemma 4.6 for details. The boundaries of $P\left(H_{\ell}\right)$ and $P\left(H_{r}\right)$ are elliptical paraboloids. Now we are prepared to state our main result.

\subsection{MAIN RESUltS}

Recall that $Z, Z_{1}, Z_{2}, \ldots$ are i.i.d. with a common distribution $\mathbb{P}_{Z}$ that satisfies Condition 4. Although we are interested in the asymptotical behavior of the random variables

$$
M_{n}=\max _{1 \leq i, j \leq n}\left|Z_{i}-Z_{j}\right|
$$

$n \geq 2$, it will be convenient to consider a very specific series of Poisson processes instead of directly investigating the random variables $M_{n}$. Being more precise, in Section 2.2 we have defined the Poisson processes $\mathbf{Z}_{n}$ in $\mathbb{R}^{d}$ with intensity measure $n \mathbb{P}_{Z}, n \in \mathbb{N}$. Now we can state our main result:

Theorem 3.5. If Conditions 1 to 4 hold, then

$$
n^{\frac{2}{d+1}}\left(2 a-\operatorname{diam}\left(\mathbf{Z}_{n}\right)\right) \stackrel{\mathcal{D}}{\longrightarrow} \min _{i, j \geq 1}\left\{\mathcal{X}_{i, 1}+\mathcal{Y}_{j, 1}-\frac{1}{4 a}\left|\tilde{\mathcal{X}}_{i}-\widetilde{\mathcal{Y}}_{j}\right|^{2}\right\}
$$

where $\left\{\mathcal{X}_{i}, i \geq 1\right\} \stackrel{\mathcal{D}}{=} \operatorname{PRM}\left(\left.p_{\ell} \cdot m_{d}\right|_{P\left(H_{\ell}\right)}\right)$ and $\left\{\mathcal{Y}_{j}, j \geq 1\right\} \stackrel{\mathcal{D}}{=} \operatorname{PRM}\left(\left.p_{r} \cdot m_{d}\right|_{P\left(H_{r}\right)}\right)$ are independent Poisson processes. The same holds true if we replace $\operatorname{diam}\left(\mathbf{Z}_{n}\right)$ with $M_{n}$. 
A special case of this result is given if we assume that $E$ is a proper ellipsoid. The following corollary illustrates that Theorem 3.5 is a generalization of the main result in Schrempp [24]:

Remark 3.6. Let $a_{1}>a_{2} \geq a_{3} \geq \ldots \geq a_{d}>0$, and put

$$
E:=\left\{z \in \mathbb{R}^{d}: \sum_{j=1}^{d}\left(\frac{z_{j}}{a_{j}}\right)^{2} \leq 1\right\}
$$

The values $a_{1}, \ldots, a_{d}$ are called the 'half-axes' of the ellipsoid $E$. Obviously, this set has a unique diameter of length $2 a_{1}$ between the points $\left(-a_{1}, \mathbf{0}\right)$ and $\left(a_{1}, \mathbf{0}\right)$; i.e. Condition 1 holds true with $a=a_{1}$. Putting $\delta_{\ell}:=\delta_{r}:=a_{1}$,

$$
O_{\ell}:=O_{r}:=\left\{\widetilde{z} \in \mathbb{R}^{d-1}: \sum_{j=2}^{d}\left(\frac{z_{j}}{a_{j}}\right)^{2}<1\right\}
$$

and

$$
s^{\ell}(\widetilde{z}):=s^{r}(\widetilde{z}):=a_{1}-a_{1} \sqrt{1-\sum_{j=2}^{d}\left(\frac{z_{j}}{a_{j}}\right)^{2}},
$$

Condition 2 is fulfilled, too. For $k, \ell \in\{2, \ldots, d\}$ we obtain

$$
\begin{aligned}
& s_{k}^{r}(\widetilde{z})=a_{1}\left(1-\sum_{j=2}^{d}\left(\frac{z_{j}}{a_{j}}\right)^{2}\right)^{-\frac{1}{2}} \cdot \frac{z_{k}}{a_{k}^{2}} \\
& s_{k \ell}^{r}(\widetilde{z})=a_{1}\left(1-\sum_{j=2}^{d}\left(\frac{z_{j}}{a_{j}}\right)^{2}\right)^{-\frac{3}{2}} \cdot \frac{z_{k}}{a_{k}^{2}} \cdot \frac{z_{\ell}}{a_{\ell}^{2}}+\delta_{k \ell} \cdot a_{1}\left(1-\sum_{j=2}^{d}\left(\frac{z_{j}}{a_{j}}\right)^{2}\right)^{-\frac{1}{2}} \cdot \frac{1}{a_{k}^{2}},
\end{aligned}
$$

and hence the Hessians $H_{\ell}$ and $H_{r}$ of $s^{\ell}$ and $s^{r}$ at the point $\mathbf{0}$ are given by

$$
H_{\ell}=H_{r}=\operatorname{diag}\left(\frac{a_{1}}{a_{2}^{2}}, \ldots, \frac{a_{1}}{a_{d}^{2}}\right) .
$$

This means that the principal curvatures of $\partial E$ at the poles are $\kappa_{j}^{i}=a_{1} / a_{j}^{2}$, and that the corresponding principal directions are $\mathbf{v}_{j}^{i}=\mathbf{e}_{j} \in \mathbb{R}^{d}, i \in\{\ell, r\}, j \in\{2, \ldots, d\}$. Recall definition (3.9) and that the eigenvectors of $H_{\ell}$ and $H_{r}$ are $\mathbf{u}_{j}^{i}=\mathbf{e}_{j-1} \in \mathbb{R}^{d-1}$ for $i \in\{\ell, r\}$ and $j \in\{2, \ldots, d\}$. Since $a_{2}<a_{1}$, we have

$$
\frac{1}{\kappa_{2}^{\ell}}+\frac{1}{\kappa_{2}^{r}}=\frac{a_{2}^{2}}{a_{1}}+\frac{a_{2}^{2}}{a_{1}}=2 \frac{a_{2}^{2}}{a_{1}}=2 a_{1}\left(\frac{a_{2}}{a_{1}}\right)^{2}<2 a_{1}=2 a .
$$


Hence, inequality $(3.10)$ holds true and thus Condition 3 is fulfilled. With

$$
\begin{aligned}
P\left(H_{\ell}\right)=P\left(H_{r}\right) & =\left\{z \in \mathbb{R}^{d}: \frac{1}{2} \widetilde{z}^{\top} H_{\ell} \widetilde{z} \leq z_{1}\right\} \\
& =\left\{z \in \mathbb{R}^{d}: \frac{1}{2} \sum_{j=2}^{d} \frac{a_{1}}{a_{j}^{2}} \cdot z_{j}^{2} \leq z_{1}\right\} \\
& =\left\{z \in \mathbb{R}^{d}: \sum_{j=2}^{d}\left(\frac{z_{j}}{a_{j}}\right)^{2} \leq \frac{2 z_{1}}{a_{1}}\right\},
\end{aligned}
$$

we can apply Theorem 3.5 for distributions in $E$ satisfying Condition 4, in accordance with Theorem 2.1 in Schrempp [24].

Corollary 3.7. If $Z$ has a uniform distribution in the ellipsoid E given in Remark 3.6, Condition 4 holds true with

$$
p_{\ell}:=p_{r}:=\frac{1}{m_{d}(E)}=\left(\frac{\pi^{\frac{d}{2}}}{\Gamma\left(\frac{d}{2}+1\right)} \prod_{i=1}^{d} a_{i}\right)^{-1}>0 .
$$

Hence, Theorem 3.5 is applicable. In the special case $d=2$ and $a_{1}=1$ we have $a_{2}<1, p_{\ell}=p_{r}=1 /\left(\pi a_{2}\right)$,

$$
P:=P\left(H_{\ell}\right)=P\left(H_{r}\right)=\left\{z \in \mathbb{R}^{2}:\left(\frac{z_{2}}{a_{2}}\right)^{2} \leq 2 z_{1}\right\},
$$

and it follows that

$$
n^{2 / 3}\left(2-M_{n}\right) \stackrel{\mathcal{D}}{\longrightarrow} \min _{i, j \geq 1}\left\{\mathcal{X}_{i, 1}+\mathcal{Y}_{j, 1}-\frac{1}{4}\left(\mathcal{X}_{i, 2}-\mathcal{Y}_{j, 2}\right)^{2}\right\},
$$

with two independent Poisson processes $\mathbf{X}=\left\{\mathcal{X}_{i}, i \geq 1\right\} \stackrel{\mathcal{D}}{=} \operatorname{PRM}\left(\left.p_{\ell} \cdot m_{2}\right|_{P}\right)$ and $\mathbf{Y}=\left\{\mathcal{Y}_{j}, j \geq 1\right\} \stackrel{\mathcal{D}}{=} \operatorname{PRM}\left(\left.p_{r} \cdot m_{2}\right|_{P}\right)$.

To illustrate the speed of convergence in Corollary 3.7, we present the result of a simulation study. To this end, define $G(x, y):=x_{1}+y_{1}-\left(x_{2}-y_{2}\right)^{2} / 4$. In the proof of Lemma 4.10 one can see that $G(x, y) \geq c\left(x_{1}+y_{1}\right),(x, y) \in P\left(H_{\ell}\right) \times P\left(H_{r}\right)$, for some fixed $c \in(0,1)$. Therefore, the probability that a point $\mathcal{X}_{i}$ with a 'large' first component $\mathcal{X}_{i, 1}$ determines the minimum above is 'small' (we omit details). The same holds for $\mathcal{Y}_{j}$. We can thus approximate the limiting distribution above by taking independent Poisson processes with intensity measures $\left.p_{\ell} \cdot m_{2}\right|_{\widetilde{P}}$ and $\left.p_{r} \cdot m_{2}\right|_{\widetilde{P}}$ where $\widetilde{P}:=P\left(H_{\ell}\right) \cap\left\{z \in \mathbb{R}^{2}: z_{1} \leq b\right\}$ for some fixed $b>0$. The larger the minor half-axis $a_{2}$ is (i.e. the more $E$ becomes 'circlelike'), the larger $b$ has to be chosen in order 
to have a good approximation of the distributional limit in (3.14) (we omit details). See Figure 3.4 for an illustration of the sets $E$ (left) and $P$ (right) and Figure 3.5 for the result of a simulation. Notice the different scalings between the left- and the right-hand image in Figure 3.4.
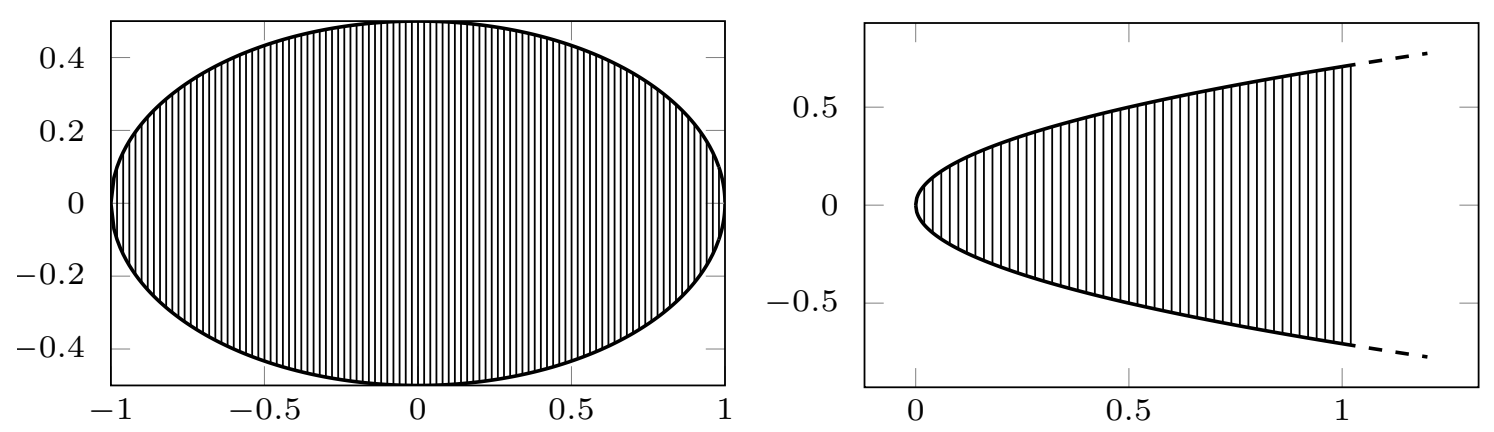

Figure 3.4: The sets $E$ (left) and $P$ (right) in the setting of Corollary 3.7 for $d=2$ with $a_{1}=1, a_{2}=1 / 2$.

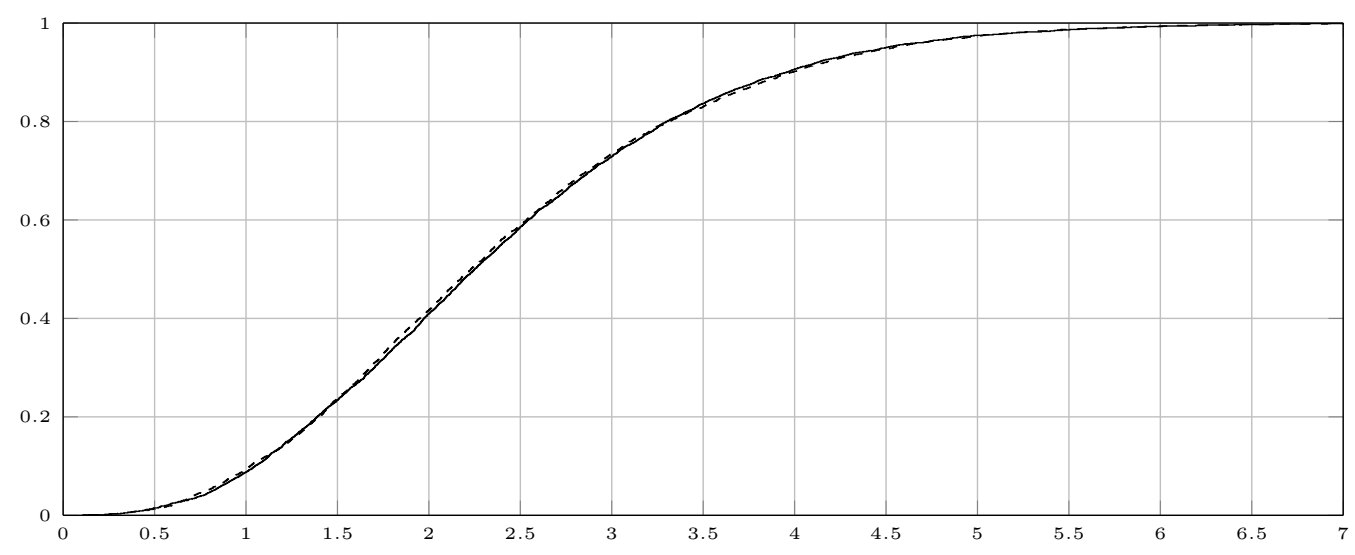

Figure 3.5: Empirical distribution function of $n^{2 / 3}\left(2-M_{n}\right)$ in the setting of Corollary 3.7 for $d=2$ with $a_{1}=1, a_{2}=1 / 2, n=1000$ (solid, 5000 replications). The limit distribution is approximated as described after Corollary 3.7 with $b=10$ (dashed, 5000 replications). 
For $i \in\{\ell, r\}$ it is possible to describe the limiting set $P\left(H_{i}\right)$ in terms of the principal curvatures and directions. Using the notation of page 22 , we have

$$
\begin{aligned}
P\left(H_{i}\right) & =\left\{\left(z_{1}, \widetilde{z}\right) \in \mathbb{R}^{d}: \frac{1}{2} \widetilde{z}^{\top} H_{i} \widetilde{z} \leq z_{1}\right\} \\
& =\left\{\left(z_{1}, \widetilde{z}\right) \in \mathbb{R}^{d}: \frac{1}{2} \widetilde{z}^{\top} U_{i} U_{i}^{\top} H_{i} U_{i} U_{i}^{\top} \widetilde{z} \leq z_{1}\right\} \\
& =\left\{\left(z_{1}, \widetilde{z}\right) \in \mathbb{R}^{d}: \frac{1}{2}\left(U_{i}^{\top} \widetilde{z}\right)^{\top} D_{i}\left(U_{i}^{\top} \widetilde{z}\right) \leq z_{1}\right\} \\
& =\left\{\left(z_{1}, U_{i} \widetilde{z}\right) \in \mathbb{R}^{d}: \frac{1}{2} \widetilde{z}^{\top} D_{i} \widetilde{z} \leq z_{1}\right\} \\
& =\left\{z_{1} \mathbf{e}_{1}+\sum_{j=2}^{d} z_{j}\left(\begin{array}{c}
0 \\
\mathbf{u}_{j}^{i}
\end{array}\right) \in \mathbb{R}^{d}: \frac{1}{2} \sum_{j=2}^{d} \kappa_{j}^{i} z_{j}^{2} \leq z_{1}\right\}
\end{aligned}
$$

and thus

$$
P\left(H_{i}\right)=\left\{z_{1} \mathbf{e}_{1}+\sum_{j=2}^{d} z_{j} \mathbf{v}_{j}^{i} \in \mathbb{R}^{d}: \frac{1}{2} \sum_{j=2}^{d} \kappa_{j}^{i} z_{j}^{2} \leq z_{1}\right\}
$$

This representation is sometimes called the "normal representation of the osculating paraboloid $P\left(H_{i}\right)^{\prime}$, and it justifies the notation of the principal curvatures $\kappa_{j}^{i}$ with indices $2, \ldots, d$ instead of $1, \ldots, d-1$. If we have $\mathbf{v}_{j}^{i}=\mathbf{e}_{j}$ for each $j \in\{2, \ldots, d\}$, we especially get

$$
P\left(H_{i}\right)=\left\{z \in \mathbb{R}^{d}: \frac{1}{2} \sum_{j=2}^{d} \kappa_{j}^{i} z_{j}^{2} \leq z_{1}\right\} .
$$

Remark 3.6 is a special case of this situation.

\subsection{Comments}

In a first step we state Condition 3 in a more convenient form:

Lemma 3.8. Condition 3 is fulfilled if, and only if, for all $\alpha, \beta \in \mathbb{R}^{d-1}$ the inequality

$$
0 \leq 2 a \eta\left(\alpha^{\top} D_{\ell} \alpha+\beta^{\top} D_{r} \beta\right)+2 \alpha^{\top} U_{\ell}^{\top} U_{r} \beta-|\alpha|^{2}-|\beta|^{2}
$$

holds true. 
Proof. Writing an arbitrary vector in $\mathbb{R}^{2(d-1)}$ as $\left(\alpha^{\top}, \beta^{\top}\right)$ with $\alpha, \beta \in \mathbb{R}^{d-1}$ leads to

$$
\begin{aligned}
& \quad 0 \leq\left(\alpha^{\top}, \beta^{\top}\right)\left(\begin{array}{cc}
2 a \eta D_{\ell}-\mathrm{I}_{d-1} & U_{\ell}^{\top} U_{r} \\
U_{r}^{\top} U_{\ell} & 2 a \eta D_{r}-\mathrm{I}_{d-1}
\end{array}\right)\left(\begin{array}{c}
\alpha \\
\beta
\end{array}\right) \\
& \Longleftrightarrow \quad 0 \leq\left(2 a \eta \alpha^{\top} D_{\ell}-\alpha^{\top}+\beta^{\top} U_{r}^{\top} U_{\ell}\right. \\
& \left.\alpha^{\top} U_{\ell}^{\top} U_{r}+2 a \eta \beta^{\top} D_{r}-\beta^{\top}\right)\left(\begin{array}{c}
\alpha \\
\beta
\end{array}\right) \\
& \Longleftrightarrow \quad 0 \leq 2 a \eta \alpha^{\top} D_{\ell} \alpha-\alpha^{\top} \alpha+\beta^{\top} U_{r}^{\top} U_{\ell} \alpha+\alpha^{\top} U_{\ell}^{\top} U_{r} \beta+2 a \eta \beta^{\top} D_{r} \beta-\beta^{\top} \beta \\
& \quad 0 \quad 0 \leq 2 a \eta\left(\alpha^{\top} D_{\ell} \alpha+\beta^{\top} D_{r} \beta\right)+2 \alpha^{\top} U_{\ell}^{\top} U_{r} \beta-|\alpha|^{2}-|\beta|^{2} .
\end{aligned}
$$

In the proofs to follow, we will need bounds for $\widetilde{z}^{\top} H_{i} \widetilde{z}$, depending on $|\widetilde{z}|, i \in\{\ell, r\}$. To this end, let $A \in \mathbb{R}^{(d-1) \times(d-1)}$ be a general symmetric matrix with (real-valued) eigenvalues $\lambda_{2} \leq \ldots \leq \lambda_{d}$ and $\left\{v_{2}, \ldots, v_{d}\right\}$ be an orthonormal basis of corresponding eigenvectors. Given any $\widetilde{z} \in \mathbb{R}^{d-1}$, we have $\widetilde{z}=\sum_{k=2}^{d}\left\langle\widetilde{z}, v_{k}\right\rangle v_{k}$ and hence

$$
\widetilde{z}^{\top} A \widetilde{z}=\left\langle\widetilde{z}, \sum_{k=2}^{d}\left\langle\widetilde{z}, v_{k}\right\rangle A v_{k}\right\rangle=\sum_{k=2}^{d} \lambda_{k}\left\langle\widetilde{z}, v_{k}\right\rangle^{2}
$$

Together with $|\widetilde{z}|^{2}=\sum_{k=2}^{d}\left\langle\widetilde{z}, v_{k}\right\rangle^{2}$ and $\lambda_{2} \leq \ldots \leq \lambda_{d}$ we obtain

$$
\lambda_{2}|\widetilde{z}|^{2} \leq \widetilde{z}^{\top} A \widetilde{z} \leq \lambda_{d}|\widetilde{z}|^{2}
$$

for each $\widetilde{z} \in \mathbb{R}^{d-1}$. So, we especially get

$$
\kappa_{2}^{i}|\widetilde{z}|^{2} \leq \widetilde{z}^{\top} H_{i} \widetilde{z} \leq \kappa_{d}^{i}|\widetilde{z}|^{2}
$$

for each $\widetilde{z} \in \mathbb{R}^{d-1}$ and $i \in\{\ell, r\}$.

Now we will show that Condition 3 really ensures the unique diameter of $E$ 'close to the poles':

Lemma 3.9. Under Conditions 2 and 3, (3.1) holds true for $E$ replaced with $E \cap$ $\left\{\left|z_{1}\right|>a-\delta\right\}$ and $\delta>0$ sufficiently small.

Proof. Since the diameter of $E$ cannot be determined by interior points, it suffices to investigate points on the boundaries $M_{\ell}$ and $M_{r}$ of the pole-caps of $E$. To this end, let $(\widetilde{x}, \widetilde{y}) \in O_{\ell} \times O_{r} \backslash\{\mathbf{0}\}$. Invoking $(3.7)$ and $(3.8)$ and putting

$$
\Xi:=\frac{1}{2}\left(\widetilde{x}^{\top} H_{\ell} \widetilde{x}+\widetilde{y}^{\top} H_{r} \widetilde{y}\right)+R_{\ell}(\widetilde{x})+R_{r}(\widetilde{y}),
$$


we get

$$
\begin{aligned}
& \left|\left(-a+s^{\ell}(\widetilde{x}), \widetilde{x}\right)-\left(a-s^{r}(\widetilde{y}), \widetilde{y}\right)\right|^{2} \\
= & \left|\left(-a+\frac{1}{2} \widetilde{x}^{\top} H_{\ell} \widetilde{x}+R_{\ell}(\widetilde{x}), \widetilde{x}\right)-\left(a-\frac{1}{2} \widetilde{y}^{\top} H_{r} \widetilde{y}-R_{r}(\widetilde{y}), \widetilde{y}\right)\right|^{2} \\
= & (-2 a+\Xi)^{2}+|\widetilde{x}-\widetilde{y}|^{2} \\
= & 4 a^{2}-4 a \Xi+\Xi^{2}+|\widetilde{x}-\widetilde{y}|^{2} \\
= & 4 a^{2}-\left(4 a \Xi-\Xi^{2}\right)+|\widetilde{x}|^{2}+|\widetilde{y}|^{2}-2 \widetilde{x}^{\top} \widetilde{y} .
\end{aligned}
$$

Lemma 3.10 will show that

$$
4 a \Xi-\Xi^{2}>2 a \eta\left(\widetilde{x}^{\top} H_{\ell} \widetilde{x}+\widetilde{y}^{\top} H_{r} \widetilde{y}\right)
$$

for every $(\widetilde{x}, \widetilde{y}) \neq \mathbf{0}$ sufficiently close to $\mathbf{0}$. Representing the points $\widetilde{x}$ and $\widetilde{y}$ in terms of the bases $\left\{\mathbf{u}_{2}^{\ell}, \ldots, \mathbf{u}_{d}^{\ell}\right\}$ and $\left\{\mathbf{u}_{2}^{r}, \ldots, \mathbf{u}_{d}^{r}\right\}$, namely $\widetilde{x}=U_{\ell} \alpha$ and $\widetilde{y}=U_{r} \beta$, (3.17) gives

$$
\begin{aligned}
& \left|\left(-a+s^{\ell}(\widetilde{x}), \widetilde{x}\right)-\left(a-s^{r}(\widetilde{y}), \widetilde{y}\right)\right|^{2} \\
< & 4 a^{2}-2 a \eta\left(\widetilde{x}^{\top} H_{\ell} \widetilde{x}+\widetilde{y}^{\top} H_{r} \widetilde{y}\right)+|\widetilde{x}|^{2}+|\widetilde{y}|^{2}-2 \widetilde{x}^{\top} \widetilde{y} \\
= & 4 a^{2}-2 a \eta\left(\alpha^{\top} U_{\ell}^{\top} H_{\ell} U_{\ell} \alpha+\beta^{\top} U_{r}^{\top} H_{r} U_{r} \beta\right)+\left|U_{\ell} \alpha\right|^{2}+\left|U_{r} \beta\right|^{2}-2 \alpha^{\top} U_{\ell}^{\top} U_{r} \beta \\
= & 4 a^{2}-2 a \eta\left(\alpha^{\top} D_{\ell} \alpha+\beta^{\top} D_{r} \beta\right)-2 \alpha^{\top} U_{\ell}^{\top} U_{r} \beta+|\alpha|^{2}+|\beta|^{2} \\
\leq & 4 a^{2} .
\end{aligned}
$$

Thus, for $\delta>0$ sufficiently small, the only pair of points in $E \cap\left\{\left|z_{1}\right|>a-\delta\right\}$ with distance $2 a$ is given by $(-a, \mathbf{0})$ and $(a, \mathbf{0})$, and the proof is finished.

It remains to prove the validity of $(3.20)$.

Lemma 3.10. For $\eta \in(0,1)$ and $(\widetilde{x}, \widetilde{y}) \neq \mathbf{0}$ sufficiently close to $\mathbf{0}$ we have

$$
4 a \Xi-\Xi^{2}>2 a \eta\left(\widetilde{x}^{\top} H_{\ell} \widetilde{x}+\widetilde{y}^{\top} H_{r} \widetilde{y}\right)
$$

Proof. Let $\varepsilon:=\frac{1-\eta}{2}>0$. Without loss of generality we assume $\widetilde{x} \neq \mathbf{0}$. For $\widetilde{x}$ sufficiently close to $\mathbf{0},(3.19)$ and $R_{\ell}(\widetilde{x})=o\left(|\widetilde{x}|^{2}\right)$ lead to

$$
\left|R_{\ell}(\widetilde{x})\right|<\frac{\varepsilon}{2} \kappa_{2}^{\ell}|\widetilde{x}|^{2} \leq \frac{\varepsilon}{2} \widetilde{x}^{\top} H_{\ell} \widetilde{x}
$$

whence

$$
\frac{1}{2} \widetilde{x}^{\top} H_{\ell} \widetilde{x}+R_{\ell}(\widetilde{x})>\frac{1}{2} \widetilde{x}^{\top} H_{\ell} \widetilde{x}-\frac{\varepsilon}{2} \widetilde{x}^{\top} H_{\ell} \widetilde{x}=\frac{1-\varepsilon}{2} \widetilde{x}^{\top} H_{\ell} \widetilde{x}
$$


By the same reasoning for $\widetilde{y}$ we get

$$
\frac{1}{2} \widetilde{y}^{\top} H_{r} \widetilde{y}+R_{r}(\widetilde{y}) \geq \frac{1-\varepsilon}{2} \widetilde{y}^{\top} H_{r} \widetilde{y}
$$

Observe that, in the line above, equality holds if $\widetilde{y}=\mathbf{0}$. Putting both inequalities together yields

$$
\Xi>\frac{1-\varepsilon}{2}\left(\widetilde{x}^{\top} H_{\ell} \widetilde{x}+\widetilde{y}^{\top} H_{r} \widetilde{y}\right)
$$

and thus

$$
4 a \Xi>2 a(1-\varepsilon)\left(\widetilde{x}^{\top} H_{\ell} \widetilde{x}+\widetilde{y}^{\top} H_{r} \widetilde{y}\right) .
$$

Since close to $\mathbf{0}$ both $\left|R_{\ell}(\widetilde{x})\right| \leq \frac{\kappa_{d}^{\ell}}{2}|\widetilde{x}|^{2}$ and $\left|R_{r}(\widetilde{y})\right| \leq \frac{\kappa_{d}^{r}}{2}|\widetilde{y}|^{2}$ hold true, $(3.19)$ gives

$$
\begin{aligned}
\Xi^{2} & =\left(\frac{1}{2}\left(\widetilde{x}^{\top} H_{\ell} \widetilde{x}+\widetilde{y}^{\top} H_{r} \widetilde{y}\right)+R_{\ell}(\widetilde{x})+R_{r}(\widetilde{y})\right)^{2} \\
& \leq\left(\frac{1}{2}\left(\kappa_{d}^{\ell}|\widetilde{x}|^{2}+\kappa_{d}^{r}|\widetilde{y}|^{2}\right)+\frac{\kappa_{d}^{\ell}}{2}|\widetilde{x}|^{2}+\frac{\kappa_{d}^{r}}{2}|\widetilde{y}|^{2}\right)^{2} \\
& =\left(\kappa_{d}^{\ell}|\widetilde{x}|^{2}+\kappa_{d}^{r}|\widetilde{y}|^{2}\right)^{2} \\
& \leq \max \left\{\kappa_{d}^{\ell}, \kappa_{d}^{r}\right\}^{2}\left(|\widetilde{x}|^{2}+|\widetilde{y}|^{2}\right)^{2} .
\end{aligned}
$$

Using $(\overline{3.19})$ again yields

$$
0 \leq \frac{\left(|\widetilde{x}|^{2}+|\widetilde{y}|^{2}\right)^{2}}{\widetilde{x}^{\top} H_{\ell} \widetilde{x}+\widetilde{y}^{\top} H_{r} \widetilde{y}} \leq \frac{\left(|\widetilde{x}|^{2}+|\widetilde{y}|^{2}\right)^{2}}{\kappa_{2}^{\ell}|\widetilde{x}|^{2}+\kappa_{2}^{r}|\widetilde{y}|^{2}}
$$

Since the fraction on the right-hand side tends to 0 as $(\widetilde{x}, \widetilde{y}) \rightarrow \mathbf{0}$ we infer

$$
\Xi^{2} \leq 2 a \varepsilon\left(\widetilde{x}^{\top} H_{\ell} \widetilde{x}+\widetilde{y}^{\top} H_{r} \widetilde{y}\right)
$$

for all $(\widetilde{x}, \widetilde{y})$ sufficiently close to $\mathbf{0}$. From $(3.21)$ and $(3.22)$ we deduce that

$$
\begin{aligned}
4 a \Xi-\Xi^{2} & >2 a(1-\varepsilon)\left(\widetilde{x}^{\top} H_{\ell} \widetilde{x}+\widetilde{y}^{\top} H_{r} \widetilde{y}\right)-2 a \varepsilon\left(\widetilde{x}^{\top} H_{\ell} \widetilde{x}+\widetilde{y}^{\top} H_{r} \widetilde{y}\right) \\
& =2 a(1-2 \varepsilon)\left(\widetilde{x}^{\top} H_{\ell} \widetilde{x}+\widetilde{y}^{\top} H_{r} \widetilde{y}\right)
\end{aligned}
$$

and since $1-2 \varepsilon=1-2 \frac{1-\eta}{2}=\eta$, the proof is finished.

Now we want to show that the matrix $A(1)$ is necessarily positive semi-definite. Otherwise, we would obtain a contradiction to Condition 1.

Lemma 3.11. Under Conditions 1 and 2 we have $A(1) \geq 0$.

Proof. Assuming $A(1) \nsupseteq 0$, there exists $z \in \mathbb{R}^{2(d-1)}$ with $z^{\top} A(1) z<0$. Then, we can 
also find an $\eta^{*}>1$ with

$$
\begin{aligned}
z^{\top} A\left(\eta^{*}\right) z & =z^{\top}\left(A(1)+2 a\left(\eta^{*}-1\right) \operatorname{diag}\left(D_{\ell}, D_{r}\right)\right) z \\
& =z^{\top} A(1) z+\left(\eta^{*}-1\right) 2 a z^{\top} \operatorname{diag}\left(D_{\ell}, D_{r}\right) z \\
& <0
\end{aligned}
$$

which entails $A\left(\eta^{*}\right) \nsupseteq 0$. Notice that $\left(\eta^{*}-1\right) 2 a z^{\top} \operatorname{diag}\left(D_{\ell}, D_{r}\right) z>0$ can be made arbitrarily small by choosing $\eta^{*}$ sufficiently close to 1 . In a similar way as in the proof of Lemma 3.10, one can show

$$
4 a \Xi-\Xi^{2}<2 a \eta^{*}\left(\widetilde{x}^{\top} H_{\ell} \widetilde{x}+\widetilde{y}^{\top} H_{r} \widetilde{y}\right)
$$

for all $(\widetilde{x}, \widetilde{y}) \neq \mathbf{0}$ sufficiently close to $\mathbf{0}$. As in the proof of Lemma 3.9 we obtain

$$
\begin{aligned}
& \left|\left(-a+s^{\ell}(\widetilde{x}), \widetilde{x}\right)-\left(a-s^{r}(\widetilde{y}), \widetilde{y}\right)\right|^{2} \\
> & 4 a^{2}-2 a \eta^{*}\left(\widetilde{x}^{\top} H_{\ell} \widetilde{x}+\widetilde{y}^{\top} H_{r} \widetilde{y}\right)+|\widetilde{x}|^{2}+|\widetilde{y}|^{2}-2 \widetilde{x}^{\top} \widetilde{y} .
\end{aligned}
$$

Because of $A\left(\eta^{*}\right) \nsupseteq 0$ we can find $\alpha, \beta \in \mathbb{R}^{d-1}$ arbitrarily close to $\mathbf{0}$ with

$$
\left(\alpha^{\top}, \beta^{\top}\right) A\left(\eta^{*}\right)\left(\begin{array}{l}
\alpha \\
\beta
\end{array}\right)<0 .
$$

Using the same transformations as in the proof of Lemma 3.8, this inequality can be rewritten to

$$
-2 a \eta^{*}\left(\alpha^{\top} D_{\ell} \alpha+\beta^{\top} D_{r} \beta\right)-2 \alpha^{\top} U_{\ell}^{\top} U_{r} \beta+|\alpha|^{2}+|\beta|^{2}>0
$$

If we choose $|\alpha|$ and $|\beta|$ small enough, we have $\widetilde{x}:=U_{\ell} \alpha \in O_{\ell}$ and $\widetilde{y}:=U_{r} \beta \in O_{r}$. Putting (3.23) and (3.24) together yields

$$
\begin{aligned}
& \left|\left(-a+s^{\ell}(\widetilde{x}), \widetilde{x}\right)-\left(a-s^{r}(\widetilde{y}), \widetilde{y}\right)\right|^{2} \\
> & 4 a^{2}-2 a \eta^{*}\left(\widetilde{x}^{\top} H_{\ell} \widetilde{x}+\widetilde{y}^{\top} H_{r} \widetilde{y}\right)+|\widetilde{x}|^{2}+|\widetilde{y}|^{2}-2 \widetilde{x}^{\top} \widetilde{y} \\
= & 4 a^{2}-2 a \eta^{*}\left(\alpha^{\top} U_{\ell}^{\top} H_{\ell} U_{\ell} \alpha+\beta^{\top} U_{r}^{\top} H_{r} U_{r} \beta\right)-2 \alpha^{\top} U_{\ell}^{\top} U_{r} \beta+\left|U_{\ell} \alpha\right|^{2}+\left|U_{r} \beta\right|^{2} \\
= & 4 a^{2}-2 a \eta^{*}\left(\alpha^{\top} D_{\ell} \alpha+\beta^{\top} D_{r} \beta\right)-2 \alpha^{\top} U_{\ell}^{\top} U_{r} \beta+|\alpha|^{2}+|\beta|^{2} \\
> & 4 a^{2} .
\end{aligned}
$$

This inequality contradicts Condition 1, and the proof is finished.

The following lemma shows that inequality (3.10) is sufficient for Condition 3: 
Lemma 3.12. If (3.10) holds true, then Condition 3 is fulfilled.

Proof. Inequality $(3.10)$ ensures the existence of an $\eta^{*} \in(0,1)$ with

$$
\frac{1}{\kappa_{2}^{\ell}}+\frac{1}{\kappa_{2}^{r}}=2 a \eta^{*}
$$

Applying (3.18) to the matrices $D_{\ell}$ and $D_{r}$ yields

$$
\begin{aligned}
& 2 a \eta^{*}\left(\alpha^{\top} D_{\ell} \alpha+\beta^{\top} D_{r} \beta\right)+2 \alpha^{\top} U_{\ell}^{\top} U_{r} \beta-|\alpha|^{2}-|\beta|^{2} \\
\geq & 2 a \eta^{*}\left(\kappa_{2}^{\ell} \alpha^{\top} \alpha+\kappa_{2}^{r} \beta^{\top} \beta\right)+2 \alpha^{\top} U_{\ell}^{\top} U_{r} \beta-|\alpha|^{2}-|\beta|^{2} \\
= & \left(\frac{1}{\kappa_{2}^{\ell}}+\frac{1}{\kappa_{2}^{r}}\right)\left(\kappa_{2}^{\ell} \alpha^{\top} \alpha+\kappa_{2}^{r} \beta^{\top} \beta\right)+2 \alpha^{\top} U_{\ell}^{\top} U_{r} \beta-|\alpha|^{2}-|\beta|^{2} \\
= & |\alpha|^{2}+|\beta|^{2}+\frac{\kappa_{2}^{\ell}}{\kappa_{2}^{r}} \alpha^{\top} \alpha+\frac{\kappa_{2}^{r}}{\kappa_{2}^{\ell}} \beta^{\top} \beta+2 \alpha^{\top} U_{\ell}^{\top} U_{r} \beta-|\alpha|^{2}-|\beta|^{2} \\
= & \frac{\kappa_{2}^{\ell}}{\kappa_{2}^{r}} \alpha^{\top} \alpha+\frac{\kappa_{2}^{r}}{\kappa_{2}^{\ell}} \beta^{\top} \beta+2 \alpha^{\top} U_{\ell}^{\top} U_{r} \beta \\
= & \frac{\kappa_{2}^{\ell}}{\kappa_{2}^{r}} \alpha^{\top} U_{\ell}^{\top} U_{\ell} \alpha+\frac{\kappa_{2}^{r}}{\kappa_{2}^{\ell}} \beta^{\top} U_{r}^{\top} U_{r} \beta+2 \alpha^{\top} U_{\ell}^{\top} U_{r} \beta \\
= & \left(\sqrt{\frac{\kappa_{2}^{\ell}}{\kappa_{2}^{r}}} U_{\ell} \alpha+\sqrt{\frac{\kappa_{2}^{r}}{\kappa_{2}^{\ell}}} U_{r} \beta\right)^{\top}\left(\sqrt{\frac{\kappa_{2}^{\ell}}{\kappa_{2}^{r}}} U_{\ell} \alpha+\sqrt{\frac{\kappa_{2}^{r}}{\kappa_{2}^{\ell}}} U_{r} \beta\right) \\
= & \left|\sqrt{\frac{\kappa_{2}^{\ell}}{\kappa_{2}^{r}}} U_{\ell} \alpha+\sqrt{\frac{\kappa_{2}^{r}}{\kappa_{2}^{\ell}}} U_{r} \beta\right|^{2} \\
\geq & 0 .
\end{aligned}
$$

Consequently, Condition 3 holds with $\eta=\eta^{*}$, see Lemma 3.8.

As mentioned before, $(3.10)$ is only sufficient for the unique diameter close to the poles, not necessary. In the following example we present a set with unique diameter between $(-a, \mathbf{0})$ and $(a, \mathbf{0})$ for which inequality $(3.10)$ is not fulfilled.

Example 3.13. For $d=3$ we put $a:=1, a_{2}^{\ell}:=\frac{1}{2}, a_{3}^{\ell}:=\frac{5}{4}, a_{2}^{r}:=\frac{5}{4}, a_{3}^{r}:=\frac{1}{2}$ and

$$
\begin{aligned}
E_{1}:= & \left\{x \in \mathbb{R}^{3}: x_{1} \leq 0,\left(\frac{x_{1}}{a}\right)^{2}+\left(\frac{x_{2}}{a_{2}^{\ell}}\right)^{2}+\left(\frac{x_{3}}{a_{3}^{\ell}}\right)^{2} \leq 1\right\} \\
& \cup\left\{y \in \mathbb{R}^{3}: y_{1}>0,\left(\frac{y_{1}}{a}\right)^{2}+\left(\frac{y_{2}}{a_{2}^{r}}\right)^{2}+\left(\frac{y_{3}}{a_{3}^{r}}\right)^{2} \leq 1\right\} .
\end{aligned}
$$

This set is an ellipsoid in $\mathbb{R}^{3}$ with half-axes $1, \frac{1}{2}$ and $\frac{5}{4}$, the right half of which has been rotated by 90 degrees around the $z_{1}$-axis. Figure 3.6 illustrates the boundary of this 
set. For $\delta>0$ we denote by $E_{2}^{\delta}$ the set $E_{1} \cap\left\{\left|z_{1}\right|>1-\delta\right\}$. So, for $0<\delta<1$, the set

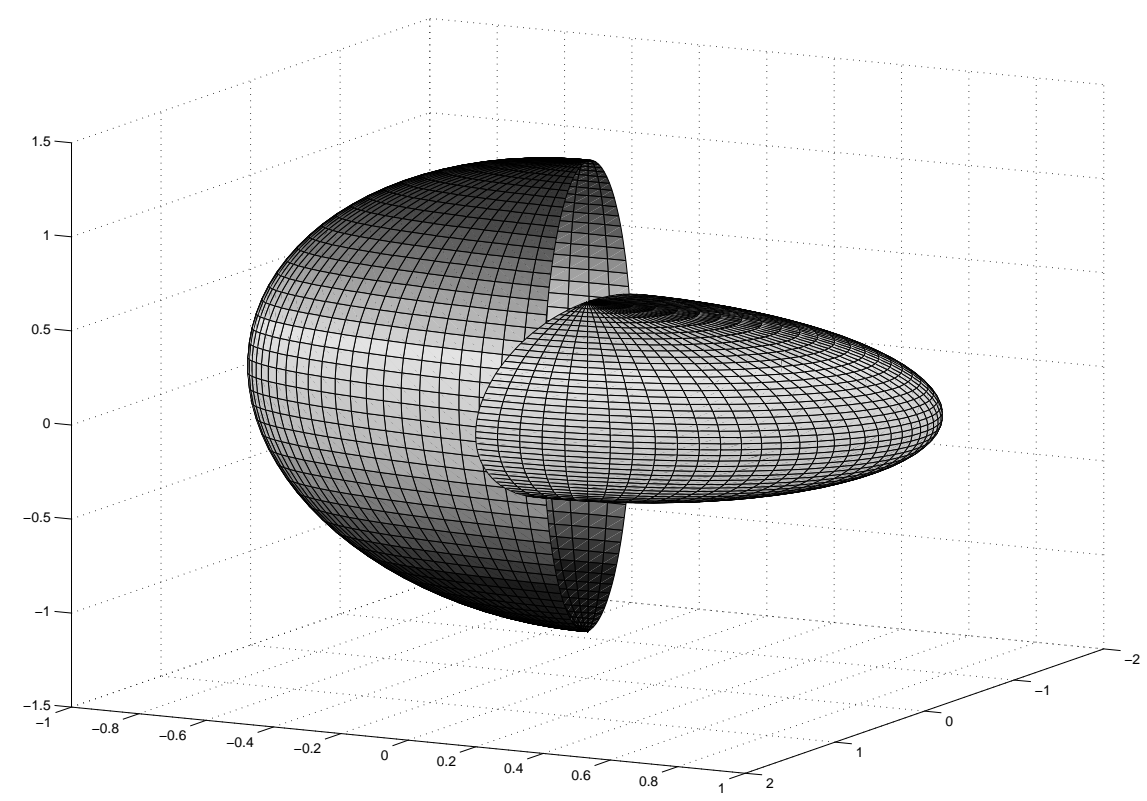

Figure 3.6: The boundary of the set $E_{1}$ given in Example 3.13.

$E_{2}^{\delta}$ consists of the pole-caps of $E_{1}$ of length $\delta$ in $z_{1}$-direction. Now we want to check whether the set $E_{2}^{\delta}$ has the necessary unique diameter between the points $(-1,0)$ and $(1, \mathbf{0})$ if we choose $\delta$ sufficiently small. The calculations seen in Remark 3.6 give

$$
\kappa_{2}^{\ell}:=\frac{a}{\left(a_{3}^{\ell}\right)^{2}}=\frac{16}{25}, \quad \kappa_{3}^{\ell}:=\frac{a}{\left(a_{2}^{\ell}\right)^{2}}=4, \quad \kappa_{2}^{r}=\frac{a}{\left(a_{2}^{r}\right)^{2}}=\frac{16}{25} \quad \text { and } \quad \kappa_{3}^{r}:=\frac{a}{\left(a_{3}^{r}\right)^{2}}=4
$$

and hence

$$
\frac{1}{\kappa_{2}^{\ell}}+\frac{1}{\kappa_{2}^{r}}=2 \cdot \frac{25}{16}=\frac{50}{16}=3.125>2=2 a .
$$

Thus, Lemma 3.12 is not applicable to the set $E_{2}^{\delta}$. But from

$$
D_{\ell}=D_{r}=\left(\begin{array}{cc}
\frac{16}{25} & 0 \\
0 & 4
\end{array}\right), \quad U_{\ell}=\left(\begin{array}{cc}
0 & 1 \\
1 & 0
\end{array}\right) \quad \text { and } \quad U_{r}=\left(\begin{array}{cc}
1 & 0 \\
0 & 1
\end{array}\right)
$$

we conclude that

$$
A(\eta)=\left(\begin{array}{cc}
2 a \eta D_{\ell}-\mathrm{I}_{d-1} & U_{\ell}^{\top} U_{r} \\
U_{r}^{\top} U_{\ell} & 2 a \eta D_{r}-\mathrm{I}_{d-1}
\end{array}\right)=\left(\begin{array}{cccc}
\frac{32}{25} \eta-1 & 0 & 0 & 1 \\
0 & 8 \eta-1 & 1 & 0 \\
0 & 1 & \frac{32}{25} \eta-1 & 0 \\
1 & 0 & 0 & 8 \eta-1
\end{array}\right) .
$$


Choosing $\eta^{*}=\frac{15}{16}$ yields $A\left(\eta^{*}\right) \geq 0$, so that Condition 3 holds true. From Lemma 3.9 we can infer that $E_{2}^{\delta}$ has a unique diameter between the points $(-1, \mathbf{0})$ and $(1, \mathbf{0})$ if $\delta$ is chosen small enough. Notice that $\operatorname{diam}\left(E_{1}\right)=\frac{5}{2}>2=|(-1, \mathbf{0})-(1, \mathbf{0})|$. Hence, the argument given above makes no sense for the set $E_{1}$ itself.

The reason why the set $E_{2}^{\delta}$ in Example 3.13 has a unique diameter between the poles without fulfilling inequality (3.10) lies in the fact that the principal directions corresponding to the principal curvatures $\kappa_{2}^{\ell}$ and $\kappa_{2}^{r}\left(\mathbf{e}_{3}\right.$ on the left pole, $\mathbf{e}_{2}$ on the right pole) are orthogonal to each other. The crucial point in this context is not the orthogonality itself but only the fact that the eigenspaces of $H_{\ell}$ to $\kappa_{2}^{\ell}$ and of $H_{r}$ to $\kappa_{2}^{r}$ are disjoint. An easy non-trivial example without this relation is given if we put $d:=3, a_{1}:=1, a_{2}:=1$ and $a_{3}:=\frac{1}{2}$ in Remark 3.6. This choice yields $\frac{1}{\kappa_{2}^{\ell}}+\frac{1}{\kappa_{2}^{r}}=2 a$, and since $E \cap\left\{z_{3}=0\right\}$ is the two-dimensional unit ball, $E$ has no unique diameter. Hence, the inequality

$$
\frac{1}{\kappa_{2}^{\ell}}+\frac{1}{\kappa_{2}^{r}}<2 a
$$

is the best possible condition to ensure the unique diameter of the underlying set close to the poles without any knowledge of the principal curvature directions. 



\section{CHAPTER 4}

\section{Proof of Theorem 3.5}

The proof of Theorem 3.5 is divided into three sections. The first one is devoted to the study of some geometric properties of the set $E$ close to the poles. In Section 4.2 we will deal with the convergence of Poisson random measures, which will be crucial for the main part of the proof of Theorem 3.5, given in Section 4.3.

\subsection{Geometric Considerations}

First of all we need some additional definitions. We shift the set $E_{\ell}$ to the right by $a \cdot \mathbf{e}_{1}$ along the $z_{1}$-axis and call this set $P_{1}\left(H_{\ell}\right)$. The set $E_{r}$ will be translated by $-a \cdot \mathbf{e}_{1}$ along the $z_{1}$-axis to the left, and it will then be reflected at the plane $\left\{z_{1}=0\right\}$. We call the resulting set $P_{1}\left(H_{r}\right)$. Looking at $(3.7)$ and $(3.8)$, we have

$$
P_{1}\left(H_{i}\right)=\left\{\left(z_{1}, \widetilde{z}\right) \in \mathbb{R}^{d}: \frac{1}{2} \widetilde{z}^{\top} H_{i} \widetilde{z}+R_{i}(\widetilde{z}) \leq z_{1}<\delta_{i}, \widetilde{z} \in O_{i}\right\}
$$

for $i \in\{\ell, r\}$. The reason underlying this construction will be seen later in (4.14). In addition to $P_{1}\left(H_{i}\right)$, we introduce the constant

$$
\widehat{\eta}:=\frac{1+\eta^{-1}}{2}
$$

based on the constant $\eta \in(0,1)$ from Condition 3. The subsequent corollary will point out two very important properties of $\widehat{\eta}$, that will be essential for the proofs to follow: 
Remark 4.1. Since $\eta \in(0,1)$, we have $\widehat{\eta}>1$, and it follows that $P\left(H_{i}\right) \varsubsetneqq \widehat{\eta} \cdot P\left(H_{i}\right)$ for $i \in\{\ell, r\}$. Without this technical expansion of the limiting sets $P\left(H_{i}\right)$, several proofs would become much more complicated. The second important property is that $\widehat{\eta}$ is not 'too large' in the sense that

$$
1-\eta \widehat{\eta}=1-\eta \frac{1+\eta^{-1}}{2}=1-\frac{\eta+1}{2}=\frac{1-\eta}{2}>0 .
$$

This inequality will be crucial for the proofs of Lemma 4.5 and Lemma 4.10.

As stated in Remark 4.1, we will need the set $\widehat{\eta} \cdot P\left(H_{i}\right)$ for $i \in\{\ell, r\}$. For later use, we give a more convenient representation of these sets:

Remark 4.2. For $i \in\{\ell, r\}$ we obtain from $(3.12)$

$$
\begin{aligned}
\widehat{\eta} \cdot P\left(H_{i}\right) & =\left\{\widehat{\eta} \cdot z \in \mathbb{R}^{d}: z \in P\left(H_{i}\right)\right\} \\
& =\left\{z \in \mathbb{R}^{d}: \widehat{\eta}^{-1} z \in P\left(H_{i}\right)\right\} \\
& =\left\{z \in \mathbb{R}^{d}: \frac{1}{2}\left(\widehat{\eta}^{-1} \widetilde{z}\right)^{\top} H_{i}\left(\widehat{\eta}^{-1} \widetilde{z}\right) \leq \widehat{\eta}^{-1} z_{1}\right\} \\
& =\left\{z \in \mathbb{R}^{d}: \frac{1}{2} \widetilde{z}^{\top} H_{i} \widetilde{z} \leq \widehat{\eta} z_{1}\right\} .
\end{aligned}
$$

In the following, we have to consider simultaneously points $x$, that are lying close to the left pole, and points $y$, lying close to the right one. For this purpose, we use the definitions of the pole-caps $E_{\ell, \delta}$ and $E_{r, \delta}$ given in (3.11) and put $E_{\delta}:=E_{\ell, \delta} \times E_{r, \delta}$ to yield

$$
E_{\delta}=\left\{(x, y) \in E_{\ell} \times E_{r}:-a \leq x_{1} \leq-a+\delta, a-\delta \leq y_{1} \leq a\right\}
$$

The next lemma shows the reason for introducing the sets $\widehat{\eta} \cdot P\left(H_{i}\right), i \in\{\ell, r\}$. The inclusion stated there will be crucial for the proof of the subsequent Lemma 4.5 and for the main part of the proof of Theorem 3.5 itself.

Lemma 4.3. There is some constant $\delta^{*} \in\left(0, \min \left\{\delta_{\ell}, \delta_{r}\right\}\right]$, so that the inclusion

$$
\left(P_{1}\left(H_{\ell}\right) \cap\left\{z_{1} \leq \delta\right\}\right) \times\left(P_{1}\left(H_{r}\right) \cap\left\{z_{1} \leq \delta\right\}\right) \subset \widehat{\eta} \cdot P\left(H_{\ell}\right) \times \widehat{\eta} \cdot P\left(H_{r}\right)
$$

holds true for each $\delta \in\left(0, \delta^{*}\right]$. In other words, we have

$$
\frac{1}{2} \widetilde{x}^{\top} H_{\ell} \widetilde{x} \leq \widehat{\eta}\left(a+x_{1}\right) \quad \text { and } \quad \frac{1}{2} \widetilde{y}^{\top} H_{r} \widetilde{y} \leq \widehat{\eta}\left(a-y_{1}\right)
$$

for all $(x, y) \in E_{\delta^{*}}$. 
Proof. Observe Remark 4.2 and the construction of $P_{1}\left(H_{\ell}\right)$ and $P_{1}\left(H_{r}\right)$ at the beginning of this section for checking the equivalence between (4.4) and (4.5). Without loss of generality we only show the first inequality of $(4.5)$ for $(x, y) \in E_{\delta}$ and $\delta>0$ sufficiently small. If $\delta<\delta_{\ell}$, it follows from (3.7) and the definition of $E_{\delta}$ that

$$
x \in\left\{\left(z_{1}, \widetilde{z}\right) \in \mathbb{R}^{d}:-a+\frac{1}{2} \widetilde{z}^{\top} H_{\ell} \widetilde{z}+R_{\ell}(\widetilde{z}) \leq z_{1} \leq-a+\delta, \widetilde{z} \in O_{l}\right\},
$$

whence

$$
\frac{1}{2} \widetilde{x}^{\top} H_{\ell} \widetilde{x}+R_{\ell}(\widetilde{x}) \leq a+x_{1} \leq \delta .
$$

As $\delta \rightarrow 0$ we get $|\widetilde{x}| \rightarrow 0$ on $E_{\delta}$, and because of $R_{\ell}(\widetilde{x})=o\left(|\widetilde{x}|^{2}\right)$ the relation $R_{\ell}(\widetilde{x})=o\left(\widetilde{x}^{\top} H_{\ell} \widetilde{x}\right)$ holds true, too. Putting $\varepsilon:=\frac{\eta^{-1}-1}{\eta^{-1}+1}>0$, we obtain for sufficiently small $\delta>0$

$$
\left|R_{\ell}(\widetilde{x})\right| \leq \frac{\varepsilon}{2} \widetilde{x}^{\top} H_{\ell} \widetilde{x}
$$

for every $(x, y) \in E_{\delta}$. Combining this inequality with $(4.6)$ shows that

$$
\frac{1-\varepsilon}{2} \widetilde{x}^{\top} H_{\ell} \widetilde{x} \leq a+x_{1}
$$

and hence, by the definition of $\widehat{\eta}$ given in $(4.2)$,

$$
\begin{aligned}
\frac{1}{2} \widetilde{x}^{\top} H_{\ell} \widetilde{x} & \leq \frac{1}{1-\varepsilon}\left(a+x_{1}\right) \\
& =\frac{1}{1-\frac{\eta^{-1}-1}{\eta^{-1}+1}}\left(a+x_{1}\right) \\
& =\frac{1}{\frac{\eta^{-1}+1-\eta^{-1}+1}{\eta^{-1}+1}}\left(a+x_{1}\right) \\
& =\frac{1+\eta^{-1}}{2}\left(a+x_{1}\right) \\
& =\widehat{\eta}\left(a+x_{1}\right) .
\end{aligned}
$$

Choosing $\delta^{*}$ in such a way that both inequalities figuring in $(4.5)$ hold true for each $(x, y) \in E_{\delta^{*}}$ finishes the proof.

In the following, we will, without loss of generality, only investigate $E_{\delta}$ for $\delta \in\left(0, \delta^{*}\right]$ to ensure the validity of 4.5$)$.

In the next step we examine the behavior of $|x-y|$ for $x$ close to the left pole of $E$ and $y$ close to the right one. For this purpose, we consider $\mathbb{R}^{2 d}$ to describe the simultaneous convergence of $x$ to the left pole of $E$ and $y$ to the right pole. 
Lemma 4.4. The second-order Taylor polynomial of $h: \mathbb{R}^{2 d} \rightarrow \mathbb{R},(x, y) \mapsto|x-y|$ at the point $\mathbf{a}:=(-a, \mathbf{0}, a, \mathbf{0}) \in \mathbb{R}^{2 d}$ is given by

$$
-x_{1}+y_{1}+\frac{1}{4 a} \sum_{k=2}^{d}\left(x_{k}-y_{k}\right)^{2}=-x_{1}+y_{1}+\frac{1}{4 a}|\widetilde{x}-\widetilde{y}|^{2} .
$$

Proof. Writing $h(x, y)=\langle x-y, x-y\rangle^{\frac{1}{2}}$, we obtain for $i \in\{1, \ldots, d\}$

$$
\begin{aligned}
& \frac{\partial h}{\partial x_{i}}(x, y)=\langle x-y, x-y\rangle^{-\frac{1}{2}}\left(x_{i}-y_{i}\right), \\
& \frac{\partial h}{\partial y_{i}}(x, y)=-\langle x-y, x-y\rangle^{-\frac{1}{2}}\left(x_{i}-y_{i}\right),
\end{aligned}
$$

and hence

$$
\nabla h(x, y)=\frac{1}{|x-y|}\left(\begin{array}{r}
x-y \\
-x+y
\end{array}\right) .
$$

For $i, j \in\{1, \ldots, d\}$, it follows that

$$
\begin{aligned}
& \frac{\partial^{2} h}{\partial x_{i} \partial x_{j}}(x, y)=-\langle x-y, x-y\rangle^{-\frac{3}{2}}\left(x_{i}-y_{i}\right)\left(x_{j}-y_{j}\right)+\langle x-y, x-y\rangle^{-\frac{1}{2}} \delta_{i j}, \\
& \frac{\partial^{2} h}{\partial x_{i} \partial y_{j}}(x, y)=\langle x-y, x-y\rangle^{-\frac{3}{2}}\left(x_{i}-y_{i}\right)\left(x_{j}-y_{j}\right)-\langle x-y, x-y\rangle^{-\frac{1}{2}} \delta_{i j} \\
& \frac{\partial^{2} h}{\partial y_{i} \partial y_{j}}(x, y)=-\langle x-y, x-y\rangle^{-\frac{3}{2}}\left(x_{i}-y_{i}\right)\left(x_{j}-y_{j}\right)+\langle x-y, x-y\rangle^{-\frac{1}{2}} \delta_{i j}
\end{aligned}
$$

Using the abbreviation $c:=\langle x-y, x-y\rangle^{-\frac{1}{2}}$, the Hessian $H(x, y)$ of $h(x, y)$ at the point $(x, y)$ is

$$
H(x, y):=c \cdot\left(\begin{array}{rr}
-c^{2} M+\mathrm{I}_{d} & c^{2} M-\mathrm{I}_{d} \\
c^{2} M-\mathrm{I}_{d} & -c^{2} M+\mathrm{I}_{d}
\end{array}\right),
$$

where $M:=(x-y)(x-y)^{\top} \in \mathbb{R}^{d \times d}$. At the point $\mathbf{a}=\left(-a \cdot \mathbf{e}_{1}, a \cdot \mathbf{e}_{1}\right)$, we get

$$
\nabla h(\mathbf{a})=\frac{1}{2 a}\left(\begin{array}{r}
-2 a \cdot \mathbf{e}_{1} \\
2 a \cdot \mathbf{e}_{1}
\end{array}\right)=\left(\begin{array}{r}
-\mathbf{e}_{1} \\
\mathbf{e}_{1}
\end{array}\right)
$$

$c=1 / 2 a, M=\operatorname{diag}\left(4 a^{2}, 0, \ldots, 0\right)$ and hence $-c^{2} M+\mathrm{I}_{d}=\operatorname{diag}\left(0, \mathrm{I}_{d-1}\right)$. Together with (4.7) we have

$$
H(\mathbf{a})=\frac{1}{2 a} \cdot\left(\begin{array}{rr}
\operatorname{diag}\left(0, \mathrm{I}_{d-1}\right) & -\operatorname{diag}\left(0, \mathrm{I}_{d-1}\right) \\
-\operatorname{diag}\left(0, \mathrm{I}_{d-1}\right) & \operatorname{diag}\left(0, \mathrm{I}_{d-1}\right)
\end{array}\right),
$$


and using the representation $\mathbf{a}=\left(-a \cdot \mathbf{e}_{1}, a \cdot \mathbf{e}_{1}\right)$ again, the second-order Taylor polynomial of $h$ at the point $\mathbf{a}$ is

$$
\begin{aligned}
& h(\mathbf{a})+\nabla h(\mathbf{a})^{\top}\left(\begin{array}{c}
x+a \cdot \mathbf{e}_{1} \\
y-a \cdot \mathbf{e}_{1}
\end{array}\right)+\frac{1}{2}\left(\begin{array}{c}
x+a \cdot \mathbf{e}_{1} \\
y-a \cdot \mathbf{e}_{1}
\end{array}\right)^{\top} H(\mathbf{a})\left(\begin{array}{c}
x+a \cdot \mathbf{e}_{1} \\
y-a \cdot \mathbf{e}_{1}
\end{array}\right) \\
= & 2 a-\left(x_{1}+a\right)+\left(y_{1}-a\right)+\frac{1}{4 a}\left(\widetilde{x}^{\top} \mathrm{I}_{d-1} \widetilde{x}-\widetilde{x}^{\top} \mathrm{I}_{d-1} \widetilde{y}-\widetilde{y}^{\top} \mathrm{I}_{d-1} \widetilde{x}+\widetilde{y}^{\top} \mathrm{I}_{d-1} \widetilde{y}\right) \\
= & -x_{1}+y_{1}+\frac{1}{4 a}|\widetilde{x}-\widetilde{y}|^{2} .
\end{aligned}
$$

As $(x, y) \rightarrow \mathbf{a}=(-a, \mathbf{0}, a, \mathbf{0})$, Lemma 4.4 implies

$$
|x-y|=-x_{1}+y_{1}+\frac{1}{4 a}|\widetilde{x}-\widetilde{y}|^{2}+R(x, y),
$$

where $R(x, y)=o\left(|(x, y)-\mathbf{a}|^{2}\right)$, uniformly on the ball of radius $r$ and center $\mathbf{a}$ as $r \rightarrow 0$. This uniform convergence holds especially on $E_{\delta}$ (given in $(4.3)$ ) as $\delta \rightarrow 0$. Putting

$$
\widetilde{G}(x, y):=\left(a+x_{1}\right)+\left(a-y_{1}\right)-\frac{1}{4 a}|\widetilde{x}-\widetilde{y}|^{2},
$$

we infer

$$
2 a-|x-y|=\widetilde{G}(x, y)-R(x, y) .
$$

Lemma 4.5. We have $R(x, y)=o(\widetilde{G}(x, y))$, uniformly on $E_{\delta}$ as $\delta \rightarrow 0$.

Proof. Notice that

$$
\frac{R(x, y)}{\widetilde{G}(x, y)}=\frac{R(x, y)}{|(x, y)-\mathbf{a}|^{2}} \cdot \frac{|(x, y)-\mathbf{a}|^{2}}{\widetilde{G}(x, y)}=o(1) \frac{|(x, y)-\mathbf{a}|^{2}}{\widetilde{G}(x, y)}
$$

as $\delta \rightarrow 0$, where $o(1)$ is uniformly on $E_{\delta}$. It remains to show that $|(x, y)-\mathbf{a}|^{2} / \widetilde{G}(x, y)$ is bounded on $E_{\delta}$ for small $\delta>0$. Assume without loss of generality that $\left|x_{1}\right| \leq\left|y_{1}\right|<a$. In view of $x \in E_{\ell}$ and $y \in E_{r}$, we get $0<a-y_{1} \leq a+x_{1}$. Consider in a first step the numerator of the right-most fraction figuring in $(4.10)$. With $(3.19)$ and Lemma 4.3 
we obtain for $(x, y) \in E_{\delta}$ and sufficiently small $\delta>0$

$$
\begin{aligned}
|(x, y)-\mathbf{a}|^{2} & =\left(a+x_{1}\right)^{2}+\left(a-y_{1}\right)^{2}+|\widetilde{x}|^{2}+|\widetilde{y}|^{2} \\
& \leq\left(a+x_{1}\right)^{2}+\left(a-y_{1}\right)^{2}+\frac{1}{\kappa_{2}^{\ell}} \widetilde{x}^{\top} H_{\ell} \widetilde{x}+\frac{1}{\kappa_{2}^{r}} \widetilde{y}^{\top} H_{r} \widetilde{y} \\
& \leq\left(a+x_{1}\right)^{2}+\left(a-y_{1}\right)^{2}+\frac{2 \widehat{\eta}}{\kappa_{2}^{\ell}}\left(a+x_{1}\right)+\frac{2 \widehat{\eta}}{\kappa_{2}^{r}}\left(a-y_{1}\right) \\
& \leq\left(a+x_{1}\right)^{2}+\left(a+x_{1}\right)^{2}+\frac{2 \widehat{\eta}}{\kappa_{2}^{\ell}}\left(a+x_{1}\right)+\frac{2 \widehat{\eta}}{\kappa_{2}^{r}}\left(a+x_{1}\right) \\
& =\left(a+x_{1}\right)\left(2\left(a+x_{1}\right)+\frac{2 \widehat{\eta}}{\kappa_{2}^{\ell}}+\frac{2 \widehat{\eta}}{\kappa_{2}^{r}}\right) .
\end{aligned}
$$

As a consequence of $(x, y) \in E_{\delta}$ and $\delta \rightarrow 0$ we get $x_{1} \rightarrow-a$, and thus the term inside the big brackets converges to $\frac{2 \widehat{\eta}}{\kappa_{2}^{\ell}}+\frac{2 \widehat{\eta}}{\kappa_{2}^{r}}$. We can conclude that there is a constant $c>0$ so that $|(x, y)-\mathbf{a}|^{2}<\left(a+x_{1}\right) \cdot c$ for every $(x, y) \in E_{\delta}$ and sufficiently small $\delta>0$.

In a second step we look at the denominator

$$
\widetilde{G}(x, y)=\left(a+x_{1}\right)+\left(a-y_{1}\right)-\frac{1}{4 a}|\widetilde{x}-\widetilde{y}|^{2}
$$

figuring in (4.10). Writing $\widetilde{x}=U_{\ell} \alpha$ and $\widetilde{y}=U_{r} \beta$, we deduce that

$$
\begin{aligned}
\widetilde{G}(x, y) & =\left(a+x_{1}\right)+\left(a-y_{1}\right)-\frac{1}{4 a}\left(|\widetilde{x}|^{2}+|\widetilde{y}|^{2}-2 \widetilde{x}^{\top} \widetilde{y}\right) \\
& =\left(a+x_{1}\right)+\left(a-y_{1}\right)-\frac{1}{4 a}\left(|\alpha|^{2}+|\beta|^{2}-2 \alpha^{\top} U_{\ell}^{\top} U_{r} \beta\right) .
\end{aligned}
$$

Inequality (3.17) now shows that

$$
\begin{aligned}
\widetilde{G}(x, y) & \geq\left(a+x_{1}\right)+\left(a-y_{1}\right)-\frac{1}{4 a} 2 a \eta\left(\alpha^{\top} D_{\ell} \alpha+\beta^{\top} D_{r} \beta\right) \\
& =\left(a+x_{1}\right)+\left(a-y_{1}\right)-\frac{1}{2} \eta\left(\widetilde{x}^{\top} U_{\ell} D_{\ell} U_{\ell}^{\top} \widetilde{x}+\widetilde{y}^{\top} U_{r} D_{r} U_{r}^{\top} \widetilde{y}\right) \\
& =\left(a+x_{1}\right)+\left(a-y_{1}\right)-\frac{1}{2} \eta\left(\widetilde{x}^{\top} H_{\ell} \widetilde{x}+\widetilde{y}^{\top} H_{r} \widetilde{y}\right),
\end{aligned}
$$


and by Lemma 4.3 we get for sufficiently small $\delta>0$

$$
\begin{aligned}
\widetilde{G}(x, y) & \geq\left(a+x_{1}\right)+\left(a-y_{1}\right)-\frac{1}{2} \eta\left(2 \widehat{\eta}\left(a+x_{1}\right)+2 \widehat{\eta}\left(a-y_{1}\right)\right) \\
& =\left(a+x_{1}\right)\left(1+\frac{a-y_{1}}{a+x_{1}}-\eta \widehat{\eta}\left(1+\frac{a-y_{1}}{a+x_{1}}\right)\right) \\
& =\left(a+x_{1}\right)(1-\eta \widehat{\eta})\left(1+\frac{a-y_{1}}{a+x_{1}}\right) .
\end{aligned}
$$

Remark 4.1 and $\frac{a-y_{1}}{a+x_{1}} \geq 0$ now yield

$$
\begin{aligned}
\widetilde{G}(x, y) & \geq\left(a+x_{1}\right) \frac{1-\eta}{2}\left(1+\frac{a-y_{1}}{a+x_{1}}\right) \\
& \geq\left(a+x_{1}\right) \frac{1-\eta}{2},
\end{aligned}
$$

where $\frac{1-\eta}{2}>0$. Putting both parts together, we have

$$
\frac{|(x, y)-\mathbf{a}|^{2}}{\widetilde{G}(x, y)} \leq \frac{\left(a+x_{1}\right) \cdot c}{\left(a+x_{1}\right) \cdot \frac{1-\eta}{2}}=\frac{2 c}{1-\eta}
$$

for every $(x, y) \in E_{\delta}$ and $\delta>0$ small enough, and the proof is finished.

\subsection{Convergence of Poisson Random measures}

In this section we will focus on the convergence of Poisson processes inside the sets $P_{1}\left(H_{i}\right)$ for $i \in\{\ell, r\}$. Lemma 4.8 will be the key to describe the asymptotical behavior of those points of $\mathbf{Z}_{n}$ lying close to one of the poles if we 'look through a suitably distorted magnifying glass' and let $n$ tend to infinity. In what follows, put

$$
\nu:=\frac{1}{d+1}
$$

and

$$
T_{n}(z):=\left(n^{2 \nu} z_{1}, n^{\nu} \widetilde{z}\right)
$$

for $n \in \mathbb{N}$ and $z=\left(z_{1}, \widetilde{z}\right) \in \mathbb{R}^{d}$.

Lemma 4.6. Suppose that, for $i \in\{\ell, r\}$, the random vector $V=\left(V_{1}, \ldots, V_{d}\right)$ has a density $g$ on $P_{1}\left(H_{i}\right) \cap\left\{z_{1} \leq \delta^{*}\right\}$ with $g(z)=p(1+o(1))$ uniformly on $P_{1}\left(H_{i}\right) \cap\left\{z_{1} \leq \delta\right\}$ as $\delta \rightarrow 0$ for some $p>0$. Then, for every bounded Borel set $B \subset \mathbb{R}^{d}$, we have $\mathbb{P}\left(T_{n}(V) \in B\right)=\kappa_{n}(B) / n$ with $\left.\kappa_{n}(B) \rightarrow p \cdot m_{d}\right|_{P\left(H_{i}\right)}(B)$ as $n \rightarrow \infty$. 
Proof. To emphasize the support of $g$, we write $g(z) \mathbb{1}\left\{z \in P_{1}\left(H_{i}\right) \cap\left\{z_{1} \leq \delta^{*}\right\}\right\}$ instead of $g(z)$. We have

$$
\Delta T_{n}(x)=\operatorname{det}\left(\operatorname{diag}\left(n^{2 \nu}, n^{\nu}, \ldots, n^{\nu}\right)\right)=n^{(d+1) \nu}=n,
$$

and therefore the random vector $T_{n}(V)$ has the density

$$
g_{n}(z)=\frac{g\left(T_{n}^{-1}(z)\right)}{n}=\frac{1}{n} g\left(\frac{z_{1}}{n^{2 \nu}}, \frac{1}{n^{\nu}} \widetilde{z}\right) \mathbb{1}\left\{z \in P_{n}\left(H_{i}\right)\right\}
$$

where $P_{n}\left(H_{i}\right):=T_{n}\left(P_{1}\left(H_{i}\right) \cap\left\{z_{1} \leq \delta^{*}\right\}\right)$. In view of (4.1) we get

$$
\begin{aligned}
P_{n}\left(H_{i}\right) & =\left\{z \in \mathbb{R}^{d}: T_{n}^{-1}(z) \in P_{1}\left(H_{i}\right) \cap\left\{z_{1} \leq \delta^{*}\right\}\right\} \\
& =\left\{z \in \mathbb{R}^{d}: \frac{1}{2}\left(\frac{1}{n^{\nu}} \widetilde{z}\right)^{\top} H_{i}\left(\frac{1}{n^{\nu}} \widetilde{z}\right)+R_{i}\left(\frac{1}{n^{\nu}} \widetilde{z}\right) \leq \frac{z_{1}}{n^{2 \nu}} \leq \delta^{*}, \frac{1}{n^{\nu}} \widetilde{z} \in O_{i}\right\} \\
& =\left\{z \in \mathbb{R}^{d}: \frac{1}{2} \widetilde{z}^{\top} H_{i} \widetilde{z}+n^{2 \nu} R_{i}\left(\frac{1}{n^{\nu}} \widetilde{z}\right) \leq z_{1} \leq n^{2 \nu} \delta^{*}, \widetilde{z} \in n^{\nu} O_{i}\right\} .
\end{aligned}
$$

Since $O_{i}$ is an open neighborhood of $\mathbf{0} \in \mathbb{R}^{d-1}$ and

$$
n^{2 \nu} R_{i}\left(\frac{1}{n^{\nu}} \widetilde{z}\right)=|\widetilde{z}|^{2} \cdot \frac{R_{i}\left(\frac{1}{n^{\nu}} \widetilde{z}\right)}{\left|\frac{1}{n^{\nu}} \widetilde{z}\right|^{2}} \rightarrow 0
$$

as $n \rightarrow \infty$ for each fixed $\widetilde{z} \in \mathbb{R}^{d-1}$, we see that $\mathbb{1}\left\{z \in P_{n}\left(H_{i}\right)\right\} \rightarrow \mathbb{1}\left\{z \in P\left(H_{i}\right)\right\}$ for almost all $z \in \mathbb{R}^{d}$. Observe that this convergence does not hold true for $z=$ $\left(z_{1}, \widetilde{z}\right) \in \mathbb{R}^{d}$ with $\frac{1}{2} \widetilde{z}^{\top} H_{i} \widetilde{z}=z_{1}$ and $R_{i}\left(\frac{1}{n^{\nu}} \widetilde{z}\right)>0$ for infinitely many $n \in \mathbb{N}$. But, since $\left\{z \in \mathbb{R}^{d}: \frac{1}{2} \widetilde{z}^{\top} H_{i} \widetilde{z}=z_{1}\right\}$ has Lebesgue measure 0 , these points will have no influence on the integrals to follow. For each Borel set $B \subset \mathbb{R}^{d}$, we have

$$
\mathbb{P}\left(T_{n}(V) \in B\right)=\int_{B} g_{n}(z) \mathrm{d} z=\frac{1}{n} \int_{B} g\left(\frac{z_{1}}{n^{2 \nu}}, \frac{1}{n^{\nu}} \widetilde{z}\right) \mathbb{1}\left\{z \in P_{n}\left(H_{i}\right)\right\} \mathrm{d} z
$$

If $B$ is bounded, $\sup \left\{z_{1}:\left(z_{1}, \widetilde{z}\right) \in B\right\} \leq i_{1}$ for some $i_{1} \in[0, \infty)$. Consequently, $\left(\frac{z_{1}}{n^{2 \nu}}, \frac{1}{n^{\nu}} \widetilde{z}\right) \in\left\{t \in \mathbb{R}^{d}: t_{1} \leq \frac{i_{1}}{n^{2 \nu}}\right\}$ for every $z \in B$. Since $g(z)=p(1+o(1))$, uniformly on $P_{1}\left(H_{i}\right) \cap\left\{z_{1} \leq \delta\right\}$ as $\delta \rightarrow 0$, we obtain $g\left(\frac{z_{1}}{n^{2 \nu}}, \frac{1}{n^{\nu}} \widetilde{z}\right)=p(1+o(1))$ uniformly on $B$ as $n \rightarrow \infty$, whence

$$
\mathbb{P}\left(T_{n}(V) \in B\right)=\frac{1}{n} \cdot p \int_{B}(1+o(1)) \mathbb{1}\left\{z \in P_{n}\left(H_{i}\right)\right\} \mathrm{d} z=: \frac{1}{n} \cdot \kappa_{n}(B) .
$$

Since $B$ is bounded and $(1+o(1)) \mathbb{1}\left\{z \in P_{n}\left(H_{i}\right)\right\} \rightarrow \mathbb{1}\left\{z \in P\left(H_{i}\right)\right\}$ for almost all 
$z \in \mathbb{R}^{d}$, the dominated convergence theorem gives

$$
\begin{aligned}
\lim _{n \rightarrow \infty} \kappa_{n}(B) & =p \int_{B} \lim _{n \rightarrow \infty}(1+o(1)) \mathbb{1}\left\{z \in P_{n}\left(H_{i}\right)\right\} \mathrm{d} z \\
& =p \int_{B} \mathbb{1}\left\{z \in P\left(H_{i}\right)\right\} \mathrm{d} z \\
& =\left.p \cdot m_{d}\right|_{P\left(H_{i}\right)}(B) .
\end{aligned}
$$

Remark 4.7. In the main part of the proof of Theorem 3.5 in Section 4.3, we will have to investigate point processes living inside the sets $P_{1}\left(H_{i}\right)$. But, contrary to the setting in Schrempp [24], the inclusion $P_{1}\left(H_{i}\right) \subset P\left(H_{i}\right)$ does not hold in general, and hence especially not $P_{n}\left(H_{i}\right) \subset P\left(H_{i}\right)$ for every $n \geq 1$. Therefore, the set $P\left(H_{i}\right)$ is in general not suitable as state space for our point processes. Letting $\mathbb{R}^{d}$ be the state space would rectify this problem, but then the proof of Lemma 4.10 would fail. So, this is the point where it becomes crucial to slightly enlarge the sets $P\left(H_{i}\right)$ via $\widehat{\eta} \cdot P\left(H_{i}\right)$. According to (4.4) and the choice of $\delta^{*}$ we have

$$
P_{1}\left(H_{i}\right) \cap\left\{z_{1} \leq \delta^{*}\right\} \subset \widehat{\eta} \cdot P\left(H_{i}\right)
$$

for $i \in\{\ell, r\}$. If $z \in \widehat{\eta} \cdot P\left(H_{i}\right)$, then $T_{n}(z)=\left(n^{2 \nu} z_{1}, n^{\nu} \widetilde{z}\right)$ and Remark 4.2 yield

$$
\frac{1}{2}\left(n^{\nu} \widetilde{z}\right)^{\top} H_{i}\left(n^{\nu} \widetilde{z}\right)=n^{2 \nu} \frac{1}{2} \widetilde{z}^{\top} H_{i} \widetilde{z} \leq \widehat{\eta} n^{2 \nu} z_{1},
$$

i.e, we have $T_{n}(z) \in \widehat{\eta} \cdot P\left(H_{i}\right)$ for every $n \geq 1$. We thus get the inclusion

$$
T_{n}\left(\widehat{\eta} \cdot P\left(H_{i}\right)\right) \subset \widehat{\eta} \cdot P\left(H_{i}\right)
$$

for each $n \geq 1$, and $(4.12)$ implies

$$
T_{n}\left(P_{1}\left(H_{i}\right) \cap\left\{z_{1} \leq \delta^{*}\right\}\right) \subset \widehat{\eta} \cdot P\left(H_{i}\right)
$$

Thus, we can use the state space $\widehat{\eta} \cdot P\left(H_{\ell}\right)$ for the point processes representing the random points near the left pole and $\widehat{\eta} \cdot P\left(H_{r}\right)$ for the corresponding processes near the right pole. In the proofs to follow, it will be very important to consider only the sets $E_{\delta}$ (given in $(4.3)$ ) with $\delta \in\left(0, \delta^{*}\right]$. Without this restriction, the point processes could 'leave' their state space, and the proof of Lemma 4.10 would fail. Since the asymptotical behavior of the maximum distance will be determined close to the poles, this restriction does not mean any loss of generality. Without Condition 3 it 
could be very complicated to find state spaces that are large enough to include the processes (close to the poles) but are also small enough to allow an adapted version of Lemma 4.10. These state spaces would have to be defined depending on (the signs of) the error functions $R_{i}$ in every direction of $\mathbb{R}^{d-1}$, we omit details.

As before, let $V=\left(V_{1}, \ldots, V_{d}\right)$ have a density $g$ on $P_{1}\left(H_{i}\right) \cap\left\{z_{1} \leq \delta^{*}\right\}$ with $g(z)=p(1+o(1))$ uniformly on $P_{1}\left(H_{i}\right) \cap\left\{z_{1} \leq \delta\right\}$ as $\delta \rightarrow 0$ for some $p>0$. For $n \in \mathbb{N}$ and some fixed $c>0$ let $\widetilde{\mathbf{V}}_{n}$ be a Poisson process with intensity measure $n c \cdot \mathbb{P}_{V}$. With independently chosen $N_{n} \stackrel{\mathcal{D}}{=} \operatorname{Po}(n c)$ and i.i.d. $\widetilde{Z}_{1}, \widetilde{Z}_{2}, \ldots$ with distribution $\mathbb{P}_{V}$, we have

$$
\widetilde{\mathbf{V}}_{n} \stackrel{\mathcal{D}}{=} \sum_{j=1}^{N_{n}} \varepsilon_{\widetilde{Z}_{j}}
$$

According to the Mapping Theorem for Poisson processes, see Last and Penrose [17, p. 38], $\mathbf{V}_{n}:=\widetilde{\mathbf{V}}_{n} \circ T_{n}^{-1}$ is a Poisson process with intensity measure $\mu_{n}:=n c \cdot \mathbb{P}_{V} \circ T_{n}^{-1}$, and the representation above yields

$$
\mathbf{V}_{n} \stackrel{\mathcal{D}}{=} \sum_{j=1}^{N_{n}} \varepsilon_{T_{n}\left(\widetilde{Z}_{j}\right)}
$$

We have $\mathbf{V}_{n} \in M_{p}\left(T_{n}\left(P_{1}\left(H_{i}\right) \cap\left\{z_{1} \leq \delta^{*}\right\}\right)\right), n \in \mathbb{N}$, and because of Remark 4.7 it follows that $\mathbf{V}_{n} \in M_{p}\left(\widehat{\eta} \cdot P\left(H_{i}\right)\right)$.

Lemma 4.8. Let $\mathbf{V}_{n}$ be defined as above. Then $\mathbf{V}_{n} \stackrel{\mathcal{D}}{\longrightarrow} \mathbf{V}$ with $\mathbf{V} \stackrel{\mathcal{D}}{=} P R M(\mu)$ and $\mu:=\left.p c \cdot m_{d}\right|_{P\left(H_{i}\right)}$.

Proof. We use Proposition 3.22 in Resnick [21, recapitulated as Theorem B.2 in Appendix B. Writing $\mathcal{I}$ for the set of finite unions of bounded open rectangles, we have to show that the conditions $\mathbb{P}(\mathbf{V}(\partial I)=0)=1$, (B.2) and (B.3) hold for every $I \in \mathcal{I}$. Because of $\mu(\partial I)=0$, the first requirement obviously holds, and an application of Lemma 4.6 gives

$$
\mu_{n}(I)=n c \cdot\left(\mathbb{P}_{V} \circ T_{n}^{-1}\right)(I)=n c \cdot \mathbb{P}\left(T_{n}(V) \in I\right)=c \kappa_{n}(I) \rightarrow \mu(I) .
$$

Since $\mathbf{V}_{n}$ and $\mathbf{V}$ are Poisson processes, we get

$$
\mathbb{P}\left(\mathbf{V}_{n}(I)=0\right)=e^{-\mu_{n}(I)} \frac{\mu_{n}(I)^{0}}{0 !}=e^{-\mu_{n}(I)} \rightarrow e^{-\mu(I)}=e^{-\mu(I)} \frac{\mu(I)^{0}}{0 !}=\mathbb{P}(\mathbf{V}(I)=0)
$$

and

$$
\mathbb{E}\left[\mathbf{V}_{n}(I)\right]=\mu_{n}(I) \rightarrow \mu(I)=\mathbb{E}[\mathbf{V}(I)]<\infty
$$




\subsection{Main part of the proof of Theorem 3.5}

Proof. As stated before, we only consider $\delta \in\left(0, \delta^{*}\right]$. Recall

$$
E_{\delta}=\left\{(x, y) \in E_{\ell} \times E_{r}:-a \leq x_{1} \leq-a+\delta, a-\delta \leq y_{1} \leq a\right\}
$$

$\delta>0$, and put

$$
I_{n}^{\delta}:=\left\{(i, j): 1 \leq i, j \leq N_{n},\left(Z_{i}, Z_{j}\right) \in E_{\delta}\right\}
$$

$n \in \mathbb{N}$. Letting

$$
M_{n}^{\delta}:=\max _{(i, j) \in I_{n}^{\delta}}\left|Z_{i}-Z_{j}\right|
$$

we obtain $\mathbb{P}\left(M_{n}^{\delta} \neq \operatorname{diam}\left(\mathbf{Z}_{n}\right)\right) \rightarrow 0$ for each $\delta>0$, since both

$$
\mathbb{P}\left(Z \in E \cap\left\{-a \leq z_{1} \leq-a+\delta\right\}\right)>0
$$

and

$$
\mathbb{P}\left(Z \in E \cap\left\{a-\delta \leq z_{1} \leq a\right\}\right)>0
$$

hold true for each $\delta>0$. Hence, it suffices to investigate $M_{n}^{\delta}$ for some fixed $\delta>0$ instead of $\operatorname{diam}\left(\mathbf{Z}_{n}\right)$.

According to $(4.9)$ and Lemma 4.5, for each $\varepsilon>0$ there is some $\delta>0$ so that

$$
\widetilde{G}(x, y)(1-\varepsilon) \leq 2 a-|x-y| \leq \widetilde{G}(x, y)(1+\varepsilon)
$$

for each $(x, y) \in E_{\delta}$. These inequalities imply

$$
n^{2 \nu}\left(2 a-M_{n}^{\delta}\right)=\min _{(i, j) \in I_{n}^{\delta}}\left\{n^{2 \nu}\left(2 a-\left|Z_{i}-Z_{j}\right|\right)\right\} \leq(1+\varepsilon) \min _{(i, j) \in I_{n}^{\delta}}\left\{n^{2 \nu} \widetilde{G}\left(Z_{i}, Z_{j}\right)\right\}
$$

and

$$
n^{2 \nu}\left(2 a-M_{n}^{\delta}\right) \geq(1-\varepsilon) \min _{(i, j) \in I_{n}^{\delta}}\left\{n^{2 \nu} \widetilde{G}\left(Z_{i}, Z_{j}\right)\right\}
$$

Putting $c_{\ell, \delta}:=\int_{E_{\ell, \delta}} f(z) \mathrm{d} z$ and $c_{r, \delta}:=\int_{E_{r, \delta}} f(z) \mathrm{d} z$, we define the independent random vectors $X, Y$ with densities $\left.c_{\ell, \delta}^{-1} f\right|_{E_{\ell, \delta}}$ and $\left.c_{r, \delta}^{-1} f\right|_{E_{r, \delta}}$, respectively. Furthermore, 
for $n \in \mathbb{N}$, we introduce the independent Poisson processes $\widehat{\mathbf{X}}_{n}$ and $\widehat{\mathbf{Y}}_{n}$ with intensity measures $n c_{\ell, \delta} \cdot \mathbb{P}_{X}$ and $n c_{r, \delta} \cdot \mathbb{P}_{Y}$, respectively. With independent random elements $N_{\ell, n}, N_{r, n}, X_{1}, X_{2}, \ldots, Y_{1}, Y_{2}, \ldots$, where $N_{\ell, n} \stackrel{\mathcal{D}}{=} \operatorname{Po}\left(n c_{\ell, \delta}\right), N_{r, n} \stackrel{\mathcal{D}}{=} \operatorname{Po}\left(n c_{r, \delta}\right), X_{1}, X_{2}, \ldots$ are i.i.d. with distribution $\mathbb{P}_{X}$ and $Y_{1}, Y_{2}, \ldots$ are i.i.d. with distribution $\mathbb{P}_{Y}$, we get

$$
\widehat{\mathbf{X}}_{n} \stackrel{\mathcal{D}}{=} \sum_{i=1}^{N_{\ell, n}} \varepsilon_{X_{i}} \quad \text { and } \quad \widehat{\mathbf{Y}}_{n} \stackrel{\mathcal{D}}{=} \sum_{j=1}^{N_{r, n}} \varepsilon_{Y_{j}} .
$$

Letting $I_{n}:=\left\{(i, j): 1 \leq i \leq N_{\ell, n}, 1 \leq j \leq N_{r, n}\right\}$, we obtain

$$
M_{n}^{\delta} \stackrel{\mathcal{D}}{=} \max _{(i, j) \in I_{n}}\left|X_{i}-Y_{j}\right|
$$

As above, the inequalities

$$
\begin{aligned}
(1-\varepsilon) \min _{(i, j) \in I_{n}}\left\{n^{2 \nu} \widetilde{G}\left(X_{i}, Y_{j}\right)\right\} & \leq n^{2 \nu}\left(2 a-\max _{(i, j) \in I_{n}}\left|X_{i}-Y_{j}\right|\right) \\
& \leq(1+\varepsilon) \min _{(i, j) \in I_{n}}\left\{n^{2 \nu} \widetilde{G}\left(X_{i}, Y_{j}\right)\right\}
\end{aligned}
$$

hold, and since $\varepsilon>0$ can be chosen arbitrarily small, it suffices to examine

$$
\min _{(i, j) \in I_{n}}\left\{n^{2 \nu} \widetilde{G}\left(X_{i}, Y_{j}\right)\right\}
$$

We get

$$
\begin{aligned}
n^{2 \nu} \widetilde{G}\left(X_{i}, Y_{j}\right) & =n^{2 \nu}\left(\left(a+X_{i, 1}\right)+\left(a-Y_{j, 1}\right)-\frac{1}{4 a}\left|\widetilde{X}_{i}-\widetilde{Y}_{j}\right|^{2}\right) \\
& =G\left(n^{2 \nu}\left(a+X_{i, 1}\right), n^{\nu} \widetilde{X}_{i}, n^{2 \nu}\left(a-Y_{j, 1}\right), n^{\nu} \widetilde{Y}_{j}\right),
\end{aligned}
$$

where

$$
G:\left\{\begin{array}{l}
\widehat{\eta} \cdot P\left(H_{\ell}\right) \times \widehat{\eta} \cdot P\left(H_{r}\right) \rightarrow \mathbb{R}_{+}, \\
(x, y) \mapsto x_{1}+y_{1}-\frac{1}{4 a}|\widetilde{x}-\widetilde{y}|^{2} .
\end{array}\right.
$$

The proof of Lemma 4.10 will show that $G(x, y) \geq 0$ for every $(x, y) \in \widehat{\eta} \cdot P\left(H_{\ell}\right) \times$ $\widehat{\eta} \cdot P\left(H_{r}\right)$. It will be important that $G$ is only defined on $\widehat{\eta} \cdot P\left(H_{\ell}\right) \times \widehat{\eta} \cdot P\left(H_{r}\right)$, not on $\mathbb{R}^{2 d}$ (see the proof of Lemma 4.10). This will be no restriction: Because of Remark 4.7 it suffices to use instead of $\mathbb{R}^{d}$ the state spaces $\widehat{\eta} \cdot P\left(H_{\ell}\right)$ and $\widehat{\eta} \cdot P\left(H_{r}\right)$ for the point processes $\mathbf{X}_{n}$ and $\mathbf{Y}_{n}$, respectively, where $\mathbf{X}_{n}$ and $\mathbf{Y}_{n}$ will be defined 
later. To this end, we introduce the Poisson processes

$$
\widetilde{\mathbf{X}}_{n}:=\sum_{i=1}^{N_{\ell, n}} \varepsilon_{\left(a+X_{i, 1}, \tilde{X}_{i}\right)} \quad \text { and } \quad \widetilde{\mathbf{Y}}_{n}:=\sum_{j=1}^{N_{r, n}} \varepsilon_{\left(a-Y_{j, 1}, \widetilde{Y}_{j}\right)}
$$

on $\left(P_{1}\left(H_{\ell}\right) \cap\left\{z_{1} \leq \delta^{*}\right\}\right) \subset \widehat{\eta} \cdot P\left(H_{\ell}\right)$ and $\left(P_{1}\left(H_{r}\right) \cap\left\{z_{1} \leq \delta^{*}\right\}\right) \subset \widehat{\eta} \cdot P\left(H_{r}\right)$, respectively. In view of Condition 4 , we can apply Lemma 4.8, and since $\widetilde{\mathbf{X}}_{n}$ and $\widetilde{\mathbf{Y}}_{n}$ are independent, we conclude that

$$
\mathbf{X}_{n}:=\widetilde{\mathbf{X}}_{n} \circ T_{n}^{-1} \stackrel{\mathcal{D}}{\longrightarrow} \mathbf{X} \quad \text { and } \quad \mathbf{Y}_{n}:=\widetilde{\mathbf{Y}}_{n} \circ T_{n}^{-1} \stackrel{\mathcal{D}}{\longrightarrow} \mathbf{Y}
$$

on $M_{p}\left(\widehat{\eta} \cdot P\left(H_{\ell}\right)\right)$ and $M_{p}\left(\widehat{\eta} \cdot P\left(H_{r}\right)\right)$, respectively, with independent point processes $\mathbf{X}:=\left\{\mathcal{X}_{i}, i \geq 1\right\} \stackrel{\mathcal{D}}{=} \operatorname{PRM}\left(\left.p_{\ell} \cdot m_{d}\right|_{P\left(H_{\ell}\right)}\right)$ and $\mathbf{Y}:=\left\{\mathcal{Y}_{j}, j \geq 1\right\} \stackrel{\mathcal{D}}{=} \operatorname{PRM}\left(\left.p_{r} \cdot m_{d}\right|_{P\left(H_{r}\right)}\right)$. Observe that an application of Lemma 4.8 to $\mathbf{X}_{n}$ yields $p=p_{\ell} / c_{\ell, \delta}, c=c_{\ell, \delta}$ and finally $\mu=\left.p c \cdot m_{d}\right|_{P\left(H_{\ell}\right)}=\left.p_{\ell} \cdot m_{d}\right|_{P\left(H_{\ell}\right)}$. By construction, we have the representations

$$
\mathbf{X}_{n}=\sum_{i=1}^{N_{\ell, n}} \varepsilon_{T_{n}\left(a+X_{i, 1}, \widetilde{X}_{i}\right)}=\sum_{i=1}^{N_{\ell, n}} \varepsilon_{\left(n^{2 \nu}\left(a+X_{i, 1}\right), n^{\nu} \widetilde{X}_{i}\right)}
$$

and

$$
\mathbf{Y}_{n}=\sum_{j=1}^{N_{r, n}} \varepsilon_{T_{n}\left(a-Y_{j, 1}, \widetilde{Y}_{j}\right)}=\sum_{j=1}^{N_{r, n}} \varepsilon_{\left(n^{2 \nu}\left(a-Y_{j, 1}\right), n^{\nu} \widetilde{Y}_{j}\right)} .
$$

According to Proposition 3.17 in Resnick [21], $M_{p}\left(\widehat{\eta} \cdot P\left(H_{\ell}\right)\right)$ and $M_{p}\left(\widehat{\eta} \cdot P\left(H_{r}\right)\right)$ are separable. By Appendix M10 in Billingsley [4] we know that $M_{p}\left(\widehat{\eta} \cdot P\left(H_{\ell}\right)\right) \times M_{p}(\widehat{\eta}$. $\left.P\left(H_{r}\right)\right)$ is separable, too, and invoking Theorem 2.8 of Billingsley [4] 4.15) implies $\mathbf{X}_{n} \times \mathbf{Y}_{n} \stackrel{\mathcal{D}}{\longrightarrow} \mathbf{X} \times \mathbf{Y}$. Define now

$$
\widehat{G}:\left\{\begin{array}{l}
M_{p}\left(\widehat{\eta} \cdot P\left(H_{\ell}\right)\right) \times M_{p}\left(\widehat{\eta} \cdot P\left(H_{r}\right)\right) \rightarrow M_{p}\left(\mathbb{R}_{+}\right), \\
\mu \mapsto \mu \circ G^{-1} .
\end{array}\right.
$$

By construction, we have the representations

$$
\begin{aligned}
\widehat{G}\left(\mathbf{X}_{n} \times \mathbf{Y}_{n}\right) & =\sum_{i=1}^{N_{\ell, n}} \sum_{j=1}^{N_{r, n}} \varepsilon_{G\left(n^{2 \nu}\left(a+X_{i, 1}\right), n^{\nu} \widetilde{X}_{i}, n^{2 \nu}\left(a-Y_{j, 1}\right), n^{\nu} \widetilde{Y}_{j}\right)}, \\
\widehat{G}(\mathbf{X} \times \mathbf{Y}) & =\sum_{i, j \geq 1} \varepsilon_{G\left(\mathcal{X}_{i}, \mathcal{Y}_{j}\right)} .
\end{aligned}
$$


Since the mapping $\widehat{G}$ is continuous (see Lemma 4.10), the continuous mapping theorem gives

$$
\widehat{G}\left(\mathbf{X}_{n} \times \mathbf{Y}_{n}\right) \stackrel{\mathcal{D}}{\longrightarrow} \widehat{G}(\mathbf{X} \times \mathbf{Y}) .
$$

For a point process $\xi$ on $\mathbb{R}_{+}$we define $t_{1}(\xi):=\min \{t \geq 0: \xi([0, t]) \geq 1\}$. The reason for introducing $t_{1}$ is the very useful relation

$$
\min _{(i, j) \in I_{n}}\left\{n^{2 \nu} \widetilde{G}\left(X_{i}, Y_{j}\right)\right\}=t_{1}\left(\widehat{G}\left(\mathbf{X}_{n} \times \mathbf{Y}_{n}\right)\right)
$$

Lemma 4.11 says that $t_{1}\left(\widehat{G}\left(\mathbf{X}_{n} \times \mathbf{Y}_{n}\right)\right) \stackrel{\mathcal{D}}{\longrightarrow} t_{1}(\widehat{G}(\mathbf{X} \times \mathbf{Y}))$ and, because of

$$
t_{1}(\widehat{G}(\mathbf{X} \times \mathbf{Y}))=\min _{i, j \geq 1}\left\{G\left(\mathcal{X}_{i}, \mathcal{Y}_{j}\right)\right\}=\min _{i, j \geq 1}\left\{\mathcal{X}_{i, 1}+\mathcal{Y}_{j, 1}-\frac{1}{4 a}\left|\widetilde{\mathcal{X}}_{i}-\widetilde{\mathcal{Y}}_{j}\right|^{2}\right\}
$$

the convergence stated in $(3.13)$ follows from $(\overline{4.13})$ as $\varepsilon \rightarrow 0$. Applying Theorem 3.2 in Mayer and Molchanov [20], recapitulated as Theorem B.3 in Appendix B.3, to the functional $\Psi\left(\mathbf{Z}_{n}\right)=2-\operatorname{diam}\left(\mathbf{Z}_{n}\right)$ shows that the same result holds true if we replace $\operatorname{diam}\left(\mathbf{Z}_{n}\right)$ with $M_{n}$.

Remark 4.9. An explanation for the definition of the rescaling function $T_{n}(z)=$ $\left(n^{2 \nu} z_{1}, n^{\nu} \widetilde{z}\right)$ with $\nu=1 /(d+1)$ can be found in the proof of Lemma 4.8: The $d$ powers of $n$ have to be chosen in such a way that their sum is 1 . This requirement implies $\Delta T_{n}(z)=n$ in the proof of Lemma 4.6, whence $\mathbb{P}\left(T_{n}(V) \in B\right)=\kappa_{n}(B) / n$. As seen in the proof of Lemma 4.8 , the factors $1 / n$ and $n$ cancel out, and only $c \kappa_{n}(B)$ remains. The reason why the first power is twice the other $d-1$ identical powers is due to the Taylor series expansion of $|x-y|$ in $(4.8)$. This fact fits exactly to the shape of $E$ near the poles, so that $P_{n}\left(H_{i}\right)=T_{n}\left(P_{1}\left(H_{i}\right) \cap\left\{z_{1} \leq \delta^{*}\right\}\right)$ can converge to the set $P\left(H_{i}\right), i \in\{\ell, r\}$ (see the proof of Lemma 4.6). Finally, from (4.14) it is clear that $n^{2 \nu}$ is the correct scaling factor.

We still have to verify the continuity of the function $\widehat{G}$ :

Lemma 4.10. The function $\widehat{G}$ is continuous.

Proof. This assertion may be proved in the same way as Proposition 3.18 in Resnick [21]. We thus only have to demonstrate that $G^{-1}(K) \subset \widehat{\eta} \cdot P\left(H_{\ell}\right) \times \widehat{\eta} \cdot P\left(H_{r}\right)$ is compact if $K \subset \mathbb{R}$ is compact. For this purpose, let $K \subset \mathbb{R}$ be compact. Since $G$ is continuous, $G^{-1}(K)$ is closed, and it remains to show that $G^{-1}(K)$ is bounded. From the specific form of $\widehat{\eta} \cdot P\left(H_{\ell}\right) \times \widehat{\eta} \cdot P\left(H_{r}\right), G^{-1}(K)$ can only be unbounded if it is unbounded in $x_{1^{-}}$or $y_{1}$-direction (at this point it is important that our state spaces for the point processes are not $\mathbb{R}^{d}$, but only the subsets $\widehat{\eta} \cdot P\left(H_{\ell}\right)$ and $\left.\widehat{\eta} \cdot P\left(H_{r}\right)\right)$. For 
fixed $(x, y) \in \widehat{\eta} \cdot P\left(H_{\ell}\right) \times \widehat{\eta} \cdot P\left(H_{r}\right)$, let $\alpha, \beta \in \mathbb{R}^{d-1}$, so that $\widetilde{x}=U_{\ell} \alpha$ and $\widetilde{y}=U_{r} \beta$. Applying the same transformations as seen for $\widetilde{G}(x, y)$ in the proof of Lemma 4.5 to $G(x, y)$ yields

$$
G(x, y) \geq x_{1}+y_{1}-\eta\left(\frac{1}{2} \widetilde{x}^{\top} H_{\ell} \widetilde{x}+\frac{1}{2} \widetilde{y}^{\top} H_{r} \widetilde{y}\right)
$$

and using the representation of $\widehat{\eta} \cdot P\left(H_{i}\right)$ given in Remark 4.2 shows that

$$
\begin{aligned}
G(x, y) & \geq x_{1}+y_{1}-\eta\left(\widehat{\eta} x_{1}+\widehat{\eta} y_{1}\right) \\
& =(1-\eta \widehat{\eta})\left(x_{1}+y_{1}\right) \\
& =\frac{1-\eta}{2}\left(x_{1}+y_{1}\right) .
\end{aligned}
$$

Since $\eta \in(0,1)$, we have $\frac{1-\eta}{2}>0$ and the assumption $(x, y) \in \widehat{\eta} \cdot P\left(H_{\ell}\right) \times \widehat{\eta} \cdot P\left(H_{r}\right)$ implies $\left(x_{1}, y_{1}\right) \in \mathbb{R}_{+}^{2}$, so that $G(x, y) \geq 0$ for each $(x, y) \in \widehat{\eta} \cdot P\left(H_{\ell}\right) \times \widehat{\eta} \cdot P\left(H_{r}\right)$. If $x_{1} \rightarrow \infty$ and/or $y_{1} \rightarrow \infty$, the lower bound $\frac{1-\eta}{2}\left(x_{1}+y_{1}\right)$ for $G(x, y)$ also tends to infinity. From the boundedness of $K$ it follows that $G^{-1}(K)$ has to be bounded in $x_{1^{-}}$and $y_{1}$-direction, too. This argument finishes the proof.

Finally, we have to prove the last lemma, used in the proof of Theorem 3.5:

Lemma 4.11. We have $t_{1}\left(\widehat{G}\left(\mathbf{X}_{n} \times \mathbf{Y}_{n}\right)\right) \stackrel{\mathcal{D}}{\longrightarrow} t_{1}(\widehat{G}(\mathbf{X} \times \mathbf{Y}))$.

Proof. In a first step we will show that $\widehat{G}(\mathbf{X} \times \mathbf{Y})(\{t\})=0$ almost surely for each $t \geq 0$. For this purpose, we consider the set

$$
G^{-1}(\{t\})=\left\{(x, y) \in \widehat{\eta} \cdot P\left(H_{\ell}\right) \times \widehat{\eta} \cdot P\left(H_{r}\right): x_{1}+y_{1}-\frac{1}{4 a}|\widetilde{x}-\widetilde{y}|^{2}=t\right\} .
$$

For some fixed $y^{*} \in \widehat{\eta} \cdot P\left(H_{r}\right)$ we define

$$
A\left(y^{*}\right):=\left\{x \in \widehat{\eta} \cdot P\left(H_{\ell}\right):\left(x, y^{*}\right) \in G^{-1}(\{t\})\right\}
$$

and obtain

$$
\begin{aligned}
A\left(y^{*}\right) & =\left\{x \in \widehat{\eta} \cdot P\left(H_{\ell}\right): x_{1}+y_{1}^{*}-\frac{1}{4 a}\left|\widetilde{x}-\widetilde{y}^{*}\right|^{2}=t\right\} \\
& =\left\{x \in \widehat{\eta} \cdot P\left(H_{\ell}\right): \sqrt{4 a\left(x_{1}-\left(t-y_{1}^{*}\right)\right)}=\left|\widetilde{x}-\widetilde{y}^{*}\right|\right\} .
\end{aligned}
$$

See Figure 4.1 for an illustration of this set. Since the set $A\left(y^{*}\right)$ has Lebesgue-measure 


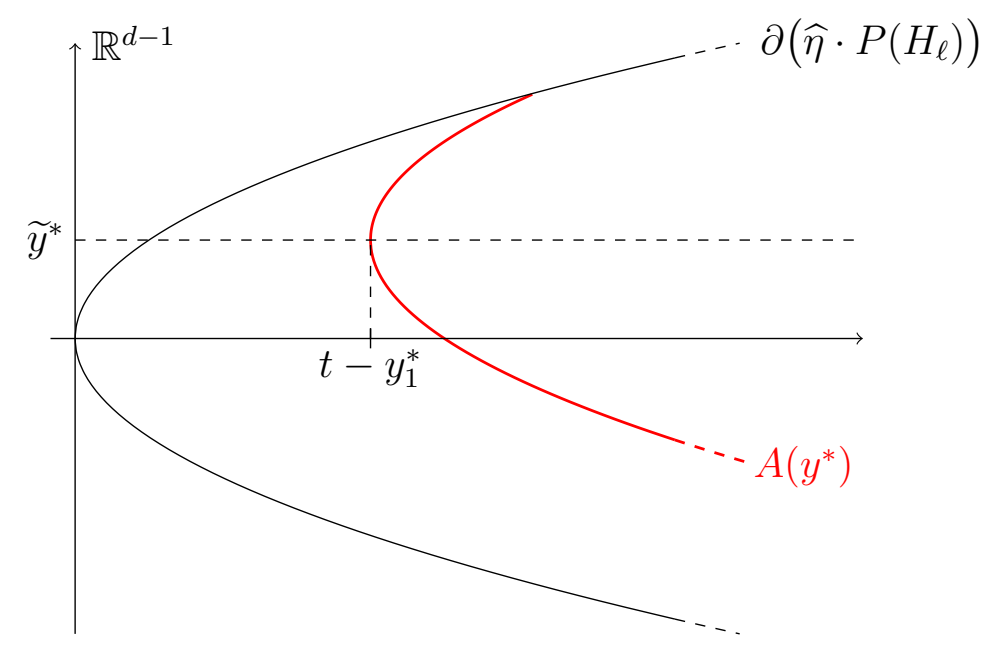

Figure 4.1: Illustration of the set $A\left(y^{*}\right)$ in the special case $y_{1}^{*}<t$ and $\widetilde{y}^{*} \neq \mathbf{0}$.

0 , we can conclude that $\mathbf{X}\left(A\left(y^{*}\right)\right)=0$ almost surely for each $y^{*} \in \widehat{\eta} \cdot P\left(H_{r}\right)$. This result implies $\widehat{G}(\mathbf{X} \times \mathbf{Y})(\{t\})=0$ almost surely for each $t \geq 0$.

In the following, we will write $\xi:=\widehat{G}(\mathbf{X} \times \mathbf{Y})$ and $\xi_{n}:=\widehat{G}\left(\mathbf{X}_{n} \times \mathbf{Y}_{n}\right)$ for $n \in \mathbb{N}$. In view of (4.17), the first part of this proof and Theorem 16.16 in Kallenberg [15], recapitulated as Theorem B.1 in Section B.3, the convergence $\xi_{n}([0, t]) \stackrel{\mathcal{D}}{\longrightarrow} \xi([0, t])$ holds true for each $t>0$. Since $\xi_{n}$ and $\xi$ are point processes, $1 / 2$ is a point of continuity of the distribution functions of both $\xi_{n}([0, t])$ and $\xi([0, t])$, and we obtain

$$
\mathbb{P}\left(\xi_{n}([0, t])=0\right)=\mathbb{P}\left(\xi_{n}([0, t]) \leq \frac{1}{2}\right) \rightarrow \mathbb{P}\left(\xi([0, t]) \leq \frac{1}{2}\right)=\mathbb{P}(\xi([0, t])=0)
$$

for each $t>0$. Thus, we have

$$
\begin{aligned}
\mathbb{P}\left(t_{1}\left(\xi_{n}\right) \leq t\right) & =1-\mathbb{P}\left(t_{1}\left(\xi_{n}\right)>t\right) \\
& =1-\mathbb{P}\left(\xi_{n}([0, t])=0\right) \\
& \rightarrow 1-\mathbb{P}(\xi([0, t])=0) \\
& =1-\mathbb{P}\left(t_{1}(\xi)>t\right) \\
& =\mathbb{P}\left(t_{1}(\xi) \leq t\right) .
\end{aligned}
$$




\section{CHAPTER 5}

\section{Generalizations 1 - Sets with unique}

DIAMETER

This chapter deals with some obvious generalizations of Theorem 3.5. Section 5.1 is devoted to more general densities than those covered by Condition 4 in Chapter 3. Being more precise, we will investigate densities supported by ellipsoids that are allowed to tend to 0 or $\infty$ close to the poles. It will turn out that the so-called Pearson Type II distributions are special distributions covered by this setting. In Section 5.2 we will take a look at more general densities supported by any set (not only ellipsoids), fulfilling the Conditions 1 to 3 . Section 5.3 establishes a limit theorem for the joint convergence of the $k$ largest distances among the random points in the settings of both Chapter 3 and Section 5.1. In Section 5.4 we adapt our results to sets that have a slightly different shape close to the poles, compared to the setting given by Condition 2. Moreover, Section 5.5 deals with $p$-superellipsoids and $p$-norms, where $1 \leq p<\infty$. If the underlying $p$-superellipsoid has a unique diameter with respect to the $p$-norm and we use this norm to define the largest distance among the random points, we obtain very similar results as seen in Chapter 3. Finally, Section 5.6 illustrates that the smoothness of the boundary of $E$ at the poles, as demanded in Section 3.1, is by no means necessary to prove results similar to that of Theorem 3.5. 


\subsection{More General DENSITIES SUPPORTED BY ELLIPSOIDS}

\subsubsection{General Setting}

In this section we consider closed ellipsoids

$$
E:=\left\{z \in \mathbb{R}^{d}: \sum_{k=1}^{d}\left(\frac{z_{k}}{a_{k}}\right)^{2} \leq 1\right\}
$$

with half axes $a_{1}>a_{2} \geq \ldots \geq a_{d}>0$, seen before in Remark 3.6. Inside of these ellipsoids we will consider distributions that are much more general than those considered in Chapter 3. For this purpose, we have to generalize Condition 4 on page 25 in a suitable way. A very wide class of elliptically symmetric distributions supported by $E$ is given by the set of all $m_{d}$-densities $f$ which can be written as

$$
f(z)=f_{1}\left(z^{\top} \Sigma^{-1} z\right) \cdot \mathbb{1}\{z \in \operatorname{int}(E)\}
$$

where $f_{1}:[0,1) \rightarrow \mathbb{R}_{+}$, and $\Sigma:=\operatorname{diag}\left(a_{1}^{2}, \ldots, a_{d}^{2}\right) \in \mathbb{R}^{d \times d}$. Notice that the definition of $f$ on $\partial E$ is completely irrelevant for our purposes. We thus assume without loss of generality $f(z)=0$ for each $z \in \partial E$ throughout this section. The asymptotic behavior of the maximum distance will depend only on the shape of $f_{1}$ close to the upper bound 1 , as long as $f_{1}(t)>0$ for each $t$ sufficiently close to 1 . We assume that $f_{1}$ behaves like a power function close to 1 with a power larger than -1 , i.e., we assume

$$
f_{1}(t) \sim \alpha(1-t)^{\beta}
$$

as $t \uparrow 1$, for some $\alpha>0$ and $\beta>-1$. Notice that the function $f$ would not be integrable - and hence be no density - if $\beta \leq-1$. More generally, we will allow this power-like behavior to be asymmetric with respect to the two pole-caps $E_{\ell, \delta}=E_{\ell} \cap\left\{z_{1} \leq-a+\delta\right\}$ and $E_{r, \delta}=E_{r} \cap\left\{a-\delta \leq z_{1}\right\}$. The generalized version of Condition 4 reads as follows:

Condition 5. We assume $f: E \rightarrow \mathbb{R}_{+}, \int_{E} f(z) \mathrm{d} z=1$ and that there are constants $\alpha_{\ell}, \alpha_{r}>0$ and $\beta_{\ell}, \beta_{r}>-1$ so that for $i \in\{\ell, r\}$, the function

$$
z \mapsto \frac{f(z)}{\alpha_{i}\left(1-z^{\top} \Sigma^{-1} z\right)^{\beta_{i}}},
$$

that maps from int $(E)$ into $\mathbb{R}_{+}$, can be extended continuously at the poles $(-a, \mathbf{0})$ and $(a, \mathbf{0})$ with value 1 . Thereby, $\alpha_{\ell}, \beta_{\ell}$ correspond to the left pole $(-a, \mathbf{0})$ and $\alpha_{r}, \beta_{r}$ to the right pole $(-a, \mathbf{0})$, respectively. 
Notice that Condition 4 was a special case of this condition, namely for $\beta_{i}=0$ and with $\alpha_{i}=p_{i}, i \in\{\ell, r\}$ (observe that we can use $E$ instead of $\operatorname{int}(E)$ in this case). To obtain a feeling for the general shape of such densities, we refer to the Figures 5.4, 5.6, 5.8 and 5.10 on the pages 72 to 74 . The left-hand image in each figure illustrates the density of a so-called Pearson Type II distribution for different values of $\beta$. These densities are a (symmetric) special case of Condition 5. Using the pole-caps $E_{\ell, \delta}=E_{\ell} \cap\left\{z_{1} \leq-a+\delta\right\}$ and $E_{r, \delta}=E_{r} \cap\left\{a-\delta \leq z_{1}\right\}$, the property of continuity assumed in Condition 5 can be rewritten as

$$
f(z)=(1+o(1)) \cdot \alpha_{i}\left(1-z^{\top} \Sigma^{-1} z\right)^{\beta_{i}}
$$

where $o(1)$ is uniformly on $\operatorname{int}\left(E_{i, \delta}\right)$ as $\delta \rightarrow 0, i \in\{\ell, r\}$. The crucial difference to the setting of Theorem 3.5 occurs in Lemma 4.6. Before we state the main result of this section, which is Theorem 5.3, we will focus on this essential difference. To this end, we need several additional (and partly very technical) definitions. As already seen in Remark 3.6, we have

$$
H_{\ell}=H_{r}=\operatorname{diag}\left(\frac{a_{1}}{a_{2}^{2}}, \ldots, \frac{a_{1}}{a_{d}^{2}}\right),
$$

and because of this symmetry, we briefly write $H:=H_{\ell}=H_{r}$. In this section, we cannot work with the representation of the set $P_{1}(H)$ given in (4.1), since we need the precise form of the error function $R_{i}$, figuring in the aforementioned equation. Remember now the construction of $P_{1}(H)$ given at the beginning of Section 4.1. In this section, we use the same construction for $\operatorname{int}(E)$ instead of $E$ to avoid divisions by 0 for $\beta<0$, and we conclude that

$$
\begin{aligned}
P_{1}(H) & =\left\{z \in \mathbb{R}^{d}:\left(\frac{z_{1}-a_{1}}{a_{1}}\right)^{2}+\sum_{k=2}^{d}\left(\frac{z_{k}}{a_{k}}\right)^{2}<1, z_{1}<a_{1}\right\} \\
& =\left\{z \in \mathbb{R}^{d}: \sum_{k=2}^{d}\left(\frac{z_{k}}{a_{k}}\right)^{2}<\frac{2 z_{1}}{a_{1}}-\left(\frac{z_{1}}{a_{1}}\right)^{2}, z_{1}<a_{1}\right\} .
\end{aligned}
$$

To show an adjusted version of Lemma 4.6, we have, in generalization of (4.11), to define the constant

$$
\nu:=\frac{1}{d+1+2 \beta},
$$


$\beta>-1$, and the rescaling function

$$
T_{n}(z):=\left(n^{2 \nu} z_{1}, n^{\nu} \widetilde{z}\right)
$$

for $n \in \mathbb{N}$ and $z=\left(z_{1}, \widetilde{z}\right) \in \mathbb{R}^{d}$. Similar to the proof of Lemma 4.6 ( $\delta^{*}$ is unnecessary in this setting), we define

$$
\begin{aligned}
P_{n}(H) & :=T_{n}\left(P_{1}(H)\right) \\
& =\left\{z \in \mathbb{R}^{d}: T_{n}^{-1}(z) \in P_{1}(H)\right\} \\
& =\left\{z \in \mathbb{R}^{d}: \sum_{k=2}^{d}\left(\frac{z_{k}}{n^{\nu} a_{k}}\right)^{2}<\frac{2 z_{1}}{n^{2 \nu} a_{1}}-\left(\frac{z_{1}}{n^{2 \nu} a_{1}}\right)^{2}, \frac{z_{1}}{n^{2 \nu}}<a_{1}\right\} \\
& =\left\{z \in \mathbb{R}^{d}: \sum_{k=2}^{d}\left(\frac{z_{k}}{a_{k}}\right)^{2}<\frac{2 z_{1}}{a_{1}}-\left(\frac{z_{1}}{n^{\nu} a_{1}}\right)^{2}, z_{1}<n^{2 \nu} a_{1}\right\} .
\end{aligned}
$$

Putting

$$
P(H):=\left\{z \in \mathbb{R}^{d}: \sum_{k=2}^{d}\left(\frac{z_{k}}{a_{k}}\right)^{2}<\frac{2 z_{1}}{a_{1}}\right\}
$$

we have $P_{n}(H) \uparrow P(H)$. For $\beta>-1$ we define the limiting density

$$
\lambda_{\beta}(z):=\left(\frac{2 z_{1}}{a_{1}}-\sum_{k=2}^{d}\left(\frac{z_{k}}{a_{k}}\right)^{2}\right)^{\beta} \mathbb{1}\{z \in P(H)\}
$$

on the limiting set $P(H)$. Moreover, for $n \in \mathbb{N}$ and $i \in\{0,1\}$ we put

$$
\lambda_{\beta, n}^{i}(z):=\left(\frac{2 z_{1}}{a_{1}}-i \cdot\left(\frac{z_{1}}{n^{\nu} a_{1}}\right)^{2}-\sum_{k=2}^{d}\left(\frac{z_{k}}{a_{k}}\right)^{2}\right)^{\beta} \mathbb{1}\left\{z \in P_{n}(H)\right\}
$$

Based on these densities, we define for $B \in \mathcal{B}^{d}$

$$
\begin{aligned}
\Lambda_{\beta}(B) & :=\int_{B} \lambda_{\beta}(z) \mathrm{d} z, \\
\Lambda_{\beta, n}^{i}(B) & :=\int_{B} \lambda_{\beta, n}^{i}(z) \mathrm{d} z .
\end{aligned}
$$

We want to give a short explanation for these very technical but necessary definitions: Under Condition 5 (and with the correctly adjusted rate of rescaling, given by the new definition of $\nu$ ), it will turn out that $\alpha_{\ell} \cdot \Lambda_{\beta_{\ell}}$ is the intensity measure of the limiting 
Poisson process $\mathbf{X}$, seen before in the proof of Theorem 3.5. The same holds true for $\alpha_{r} \cdot \Lambda_{\beta_{r}}$ and the Poisson process $\mathbf{Y}$. The measure $\Lambda_{\beta, n}^{1}$ will occur very naturally in the proof of the following Lemma 5.1. Observe that $\lambda_{\beta, n}^{0}(z)=\lambda_{\beta}(z) \mathbb{1}\left\{z \in P_{n}(H)\right\}$ for each $n \in \mathbb{N}$. This means that $\Lambda_{\beta, n}^{0}$ is the restriction of the limiting measure $\Lambda_{\beta}$ to the subset $P_{n}(H)$ of $P(H)$ for $n \in \mathbb{N}$. This restriction of the measure $\Lambda_{\beta}$ will be very important for the proof of Lemma 5.1. Looking at the definitions of $\lambda_{\beta, n}^{1}$ and $\lambda_{\beta}$, it is obvious that $\lambda_{\beta, n}^{1}(z) \rightarrow \lambda_{\beta}(z)$ for almost all $z \in \mathbb{R}^{d}$. In the proof of Lemma 5.1, we will (basically) have to show that for every bounded Borel set $B \subset \mathbb{R}^{d}$ the equality

$$
\lim _{n \rightarrow \infty} \int_{B} \lambda_{\beta, n}^{1}(z) \mathrm{d} z=\int_{B} \lim _{n \rightarrow \infty} \lambda_{\beta, n}^{1}(z) \mathrm{d} z
$$

holds true and hence

$$
\lim _{n \rightarrow \infty} \Lambda_{\beta, n}^{1}(B)=\lim _{n \rightarrow \infty} \int_{B} \lambda_{\beta, n}^{1}(z) \mathrm{d} z=\int_{B} \lim _{n \rightarrow \infty} \lambda_{\beta, n}^{1}(z) \mathrm{d} z=\int_{B} \lambda_{\beta}(z) \mathrm{d} z=\Lambda_{\beta}(B) .
$$

Proving (5.7) will be very technical, since we can (in general) neither apply the dominated convergence theorem, nor the monotone convergence theorem. We want to illustrate this assertion: The 'difficult case' is given by $\beta<0$ and

$$
m_{d}\left(B \cap\left(P(H) \backslash P_{n}(H)\right)\right)>0
$$

for each $n \in \mathbb{N}$. Figure 5.1 illustrates such a set $B$ in the case $d=2$. For $n \in \mathbb{N}$

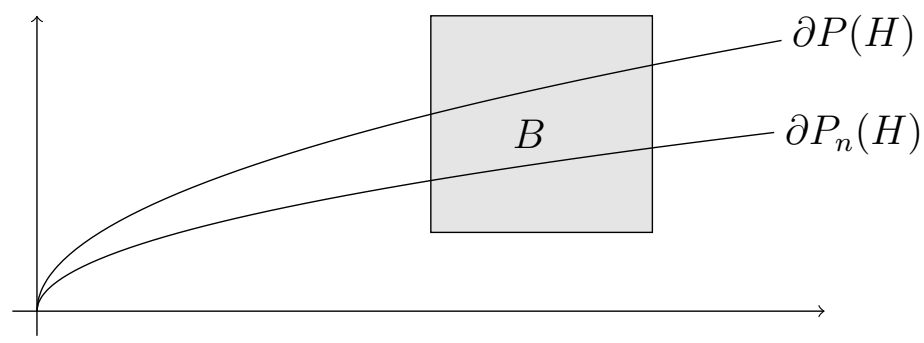

Figure 5.1: Illustration of a set $B$ satisfying $(5.8)$ in the case $d=2$

we define the set $B_{n}:=B \cap\left(P(H) \backslash P_{n}(H)\right)$, see Figure 5.2 for an illustration of this set. For each $n \in \mathbb{N}$ and $\beta<0$, we have $\lambda_{\beta, n}^{1}\left(z_{k}\right) \rightarrow \infty$ as $k \rightarrow \infty$, if $\left(z_{k}\right)_{k \geq 1}$ is a sequence in $P_{n}(H)$ with $z_{k} \rightarrow z_{0} \in \partial P_{n}(H)$ as $k \rightarrow \infty$. Such a sequence has been illustrated in Figure 5.2, too. Observing $P_{n}(H) \uparrow P(H)$ makes clear that for some fixed $n_{0} \in \mathbb{N}$ the only upper bound for $\lambda_{\beta, n_{0}}^{1}, \lambda_{\beta, n_{0}+1}^{1}, \ldots$ on $B_{n_{0}}$ is given by $\infty$. Since this assertion and $m_{d}\left(B_{n_{0}}\right)>0$ hold true for every $n_{0} \in \mathbb{N}$, it is impossible to apply the dominated convergence theorem to show (5.7). If $\beta<0$ and $z \in P(H)$, the 


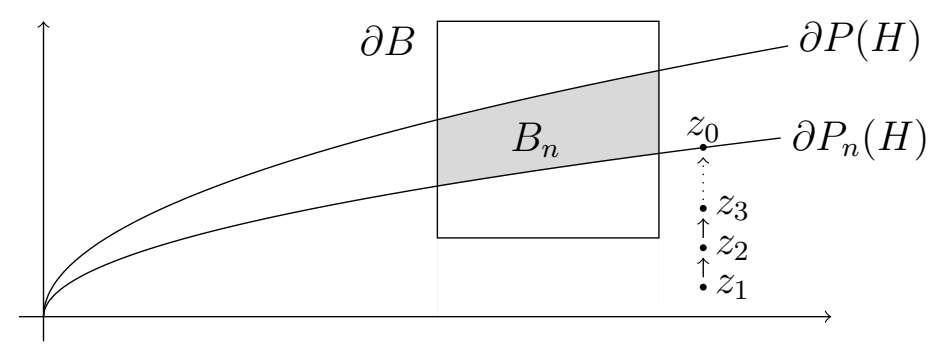

Figure 5.2: Illustration of the set $B_{n}$ and an exemplary sequence $\left(z_{k}\right)_{k \geq 1}$ in the case $d=2$

sequence $\left(\lambda_{\beta, n}^{1}\left(z_{1}\right)\right)_{n \geq 1}$ is not monotonically increasing (we will see this in the proof of Lemma 5.1). We thus cannot apply the monotone convergence theorem either to verify (5.7). The key to success will be an application of Scheffé's Lemma in the proof of the following lemma.

After all these considerations, we are prepared to state and prove an adapted version of Lemma 4.6.

Lemma 5.1. Suppose the random vector $V=\left(V_{1}, \ldots, V_{d}\right)$ has a density $g$ on $P_{1}(H)$ satisfying

$$
g(z)=\widehat{g}(z) \cdot \alpha\left(1-\left(\frac{z_{1}-a_{1}}{a_{1}}\right)^{2}-\sum_{k=2}^{d}\left(\frac{z_{k}}{a_{k}}\right)^{2}\right)^{\beta}
$$

with $\widehat{g}(z)=1+o(1)$ uniformly on $P_{1}(H) \cap\left\{z_{1} \leq \delta\right\}$ as $\delta \rightarrow 0$, for some $\alpha>0$ and $\beta>-1$. Then, for every bounded Borel set $B \subset \mathbb{R}^{d}$, we have

$$
\mathbb{P}\left(T_{n}(V) \in B\right)=\frac{\alpha}{n} \cdot \kappa_{n}(B)
$$

with $\kappa_{n}(B) \rightarrow \Lambda_{\beta}(B)$.

Proof. For clarity's sake, some technical details of this proof have been postponed to Subsection 5.1.3. To emphasize the support of $g$, we write $g(z) \mathbb{1}\left\{z \in P_{1}(H)\right\}$ instead of $g(z)$. We have

$$
\Delta T_{n}(x)=\operatorname{det}\left(\operatorname{diag}\left(n^{2 \nu}, n^{\nu}, \ldots, n^{\nu}\right)\right)=n^{(d+1) \nu}
$$

and therefore the random vector $T_{n}(V)$ has the density

$$
g_{n}(z)=\frac{g\left(T_{n}^{-1}(z)\right)}{n^{(d+1) \nu}}=\frac{1}{n^{(d+1) \nu}} g\left(\frac{z_{1}}{n^{2 \nu}}, \frac{1}{n^{\nu}} \widetilde{z}\right) \mathbb{1}\left\{z \in P_{n}(H)\right\},
$$


with $P_{n}(H)=T_{n}\left(P_{1}(H)\right)$ given in (5.3). As in the proof of Lemma 4.6, we get $\mathbb{1}\left\{z \in P_{n}(H)\right\} \rightarrow \mathbb{1}\{z \in P(H)\}$ for almost all $z \in \mathbb{R}^{d}$, and for each Borel set $B \subset \mathbb{R}^{d}$, we have

$$
\mathbb{P}\left(T_{n}(V) \in B\right)=\int_{B} g_{n}(z) \mathrm{d} z=\frac{1}{n^{(d+1) \nu}} \int_{B} g\left(\frac{z_{1}}{n^{2 \nu}}, \frac{1}{n^{\nu}} \widetilde{z}\right) \mathbb{1}\left\{z \in P_{n}(H)\right\} \mathrm{d} z .
$$

In view of $(5.9)$, we obtain

$$
\begin{aligned}
& \mathbb{P}\left(T_{n}(V) \in B\right) \\
= & \frac{1}{n^{(d+1) \nu}} \int_{B} \widehat{g}\left(\frac{z_{1}}{n^{2 \nu}}, \frac{1}{n^{\nu}} \widetilde{z}\right) \alpha\left(1-\left(\frac{\frac{z_{1}}{n^{2 \nu}}-a_{1}}{a_{1}}\right)^{2}-\sum_{k=2}^{d}\left(\frac{\frac{z_{k}}{n^{\nu}}}{a_{k}}\right)^{2}\right)^{\beta} \mathbb{1}\left\{z \in P_{n}(H)\right\} \mathrm{d} z \\
= & \frac{\alpha}{n^{(d+1+2 \beta) \nu}} \cdot \int_{B} \widehat{g}\left(\frac{z_{1}}{n^{2 \nu}}, \frac{1}{n^{\nu}} \widetilde{z}\right) \cdot \lambda_{\beta, n}^{1}(z) \mathrm{d} z .
\end{aligned}
$$

Here, the last equality follows from the definition of $\lambda_{\beta, n}^{1}$ given in $(5.5)$ as well as

$$
\begin{aligned}
& \left(1-\left(\frac{\frac{z_{1}}{n^{2 \nu}}-a_{1}}{a_{1}}\right)^{2}-\sum_{k=2}^{d}\left(\frac{\frac{z_{k}}{n^{\nu}}}{a_{k}}\right)^{2}\right)^{\beta} \\
= & \left(1-\left(\frac{z_{1}}{n^{2 \nu} a_{1}}\right)^{2}+\frac{2 z_{1}}{n^{2 \nu} a_{1}}-1-\sum_{k=2}^{d}\left(\frac{z_{k}}{n^{\nu} a_{k}}\right)^{2}\right)^{\beta} \\
= & \frac{1}{n^{2 \nu \beta}} \cdot\left(\frac{2 z_{1}}{a_{1}}-\left(\frac{z_{1}}{n^{\nu} a_{1}}\right)^{2}-\sum_{k=2}^{d}\left(\frac{z_{k}}{a_{k}}\right)^{2}\right)^{\beta}
\end{aligned}
$$

and the definition of $\nu$, which yields $n^{(d+1+2 \beta) \nu}=n$. Defining

$$
\kappa_{n}(B):=\int_{B} \widehat{g}\left(\frac{z_{1}}{n^{2 \nu}}, \frac{1}{n^{\nu}} \widetilde{z}\right) \cdot \lambda_{\beta, n}^{1}(z) \mathrm{d} z,
$$

we have to show $\kappa_{n}(B) \rightarrow \Lambda_{\beta}(B)$. Since $B$ is bounded, we especially have $\sup \left\{z_{1}\right.$ : $z \in B\}<\infty$ and hence, for each $\varepsilon>0$ we can find some $n_{0} \in \mathbb{N}$ with

$$
1-\varepsilon \leq \widehat{g}\left(\frac{z_{1}}{n^{2 \nu}}, \frac{1}{n^{\nu}} \widetilde{z}\right) \leq 1+\varepsilon
$$

for each $z \in B$ and $n \geq n_{0}$ (remember that $\widehat{g}(z)=1+o(1)$ uniformly on $P_{1}(H) \cap$ $\left\{z_{1} \leq \delta\right\}$ as $\left.\delta \rightarrow 0\right)$. Using again $\sup \left\{z_{1}: z \in B\right\}<\infty$, we can find some $t>0$ with 
$B \subset I:=\left\{z_{1} \leq t\right\}$, and from Lemma 5.6 we can conclude that

$$
\int_{B} \lambda_{\beta, n}^{1}(z) \mathrm{d} z=\Lambda_{\beta, n}^{1}(B) \leq \Lambda_{\beta, n}^{1}(I)<\infty
$$

for sufficiently large $n$. Putting both parts together we obtain

$$
(1-\varepsilon) \cdot \Lambda_{\beta, n}^{1}(B) \leq \kappa_{n}(B) \leq(1+\varepsilon) \cdot \Lambda_{\beta, n}^{1}(B)
$$

for sufficiently large $n$. Since $\varepsilon>0$ can be chosen arbitrarily small, we can focus on $\Lambda_{\beta, n}^{1}(B)$ in the following. Using Lemma 5.6 again, we see that $\int_{B} \lambda_{\beta, n}^{1}(z) \mathrm{d} z<\infty$ for sufficiently large $n, \int_{B} \lambda_{\beta}(z) \mathrm{d} z<0$ and, as mentioned before, we have $\lambda_{\beta, n}^{1}(z) \rightarrow \lambda_{\beta}(z)$ for almost all $z \in \mathbb{R}^{d}$. If we can additionally prove

$$
\int_{B}\left|\lambda_{\beta, n}^{1}(z)-\lambda_{\beta}(z)\right| \mathrm{d} z \rightarrow 0
$$

we can apply Scheffé's Lemma (in its version for positive, integrable functions, not for probability densities, see Williams [27, p. 55]) to show

$$
\lim _{n \rightarrow \infty} \Lambda_{\beta, n}^{1}(B)=\lim _{n \rightarrow \infty} \int_{B} \lambda_{\beta, n}^{1}(z) \mathrm{d} z=\int_{B} \lim _{n \rightarrow \infty} \lambda_{\beta, n}^{1}(z) \mathrm{d} z=\int_{B} \lambda_{\beta}(z) \mathrm{d} z=\Lambda_{\beta}(B) .
$$

In order to prove $(5.12)$, we again use the set $I=\left\{z_{1} \leq t\right\} \supset B$ and obtain

$$
\int_{B}\left|\lambda_{\beta, n}^{1}(z)-\lambda_{\beta}(z)\right| \mathrm{d} z \leq \int_{I}\left|\lambda_{\beta, n}^{1}(z)-\lambda_{\beta}(z)\right| \mathrm{d} z .
$$

For $n \in \mathbb{N}$ we introduce the sets

$$
\begin{aligned}
I_{1, n} & :=I \cap P_{n}(H), \\
I_{2, n} & :=I \cap\left(P(H) \backslash P_{n}(H)\right), \\
I_{3} & :=I \backslash P(H),
\end{aligned}
$$

see Figure 5.3 for an illustration of these sets in the case $d=2$. Since the inequality

$$
\frac{2 z_{1}}{a_{1}}-\left(\frac{z_{1}}{n^{\nu} a_{1}}\right)^{2}-\sum_{k=2}^{d}\left(\frac{z_{k}}{a_{k}}\right)^{2} \leq \frac{2 z_{1}}{a_{1}}-\sum_{k=2}^{d}\left(\frac{z_{k}}{a_{k}}\right)^{2}
$$




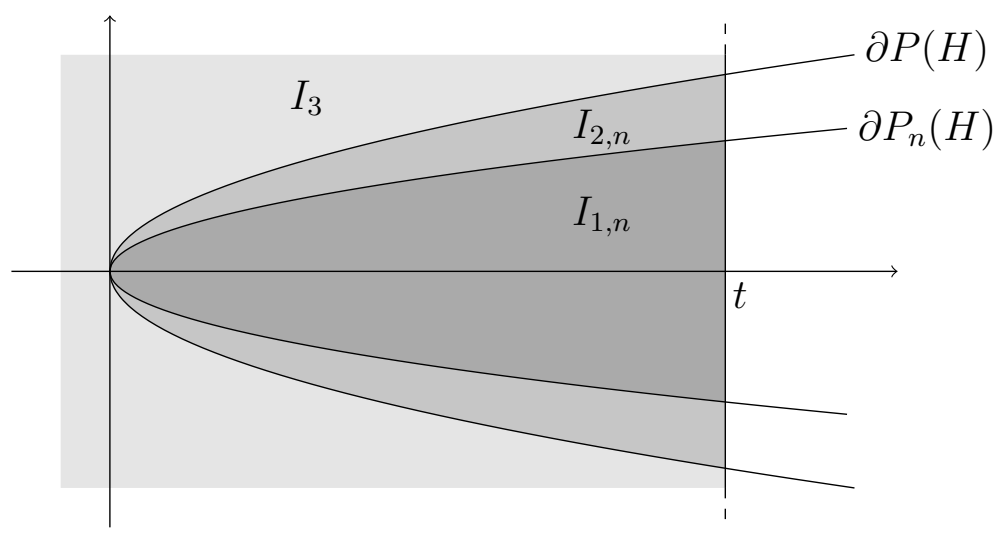

Figure 5.3: Illustration of the sets $I_{1, n}, I_{2, n}$ and $I_{3}$ in the case $d=2$

holds for each $z \in P_{n}(H)$, the definitions given in (5.4) and (5.5) yield

$$
\begin{cases}\lambda_{\beta, n}^{1}(z) \leq \lambda_{\beta}(z), & \text { if } \beta \geq 0 \\ \lambda_{\beta, n}^{1}(z) \geq \lambda_{\beta}(z), & \text { if } \beta<0\end{cases}
$$

for each $z \in P_{n}(H)$. Hence, for each $n \in \mathbb{N}$ we get

$$
\begin{cases}0<\lambda_{\beta, n}^{1}(z) \leq \lambda_{\beta}(z), & \text { if } z \in I_{1, n} \text { and } \beta \geq 0 \\ \lambda_{\beta, n}^{1}(z) \geq \lambda_{\beta}(z)>0, & \text { if } z \in I_{1, n} \text { and } \beta<0 \\ \lambda_{\beta, n}^{1}(z)=0,0<\lambda_{\beta}(z), & \text { if } z \in I_{2, n}, \\ \lambda_{\beta, n}^{1}(z)=\lambda_{\beta}(z)=0, & \text { if } z \in I_{3} .\end{cases}
$$

This consideration and (5.5) allow us to compute the integral figuring on the righthand side of (5.13) via

$$
\begin{aligned}
& \int_{I}\left|\lambda_{\beta, n}^{1}(z)-\lambda_{\beta}(z)\right| \mathrm{d} z \\
= & \int_{I_{1, n}}\left|\lambda_{\beta, n}^{1}(z)-\lambda_{\beta}(z)\right| \mathrm{d} z+\int_{I_{2, n}}\left|\lambda_{\beta, n}^{1}(z)-\lambda_{\beta}(z)\right| \mathrm{d} z+\int_{I_{3}}\left|\lambda_{\beta, n}^{1}(z)-\lambda_{\beta}(z)\right| \mathrm{d} z \\
= & (-1)^{\mathbb{1}\{\beta \geq 0\}} \int_{I_{1, n}}\left(\lambda_{\beta, n}^{1}(z)-\lambda_{\beta}(z)\right) \mathrm{d} z+\int_{I_{2, n}} \lambda_{\beta}(z) \mathrm{d} z \\
= & (-1)^{\mathbb{1}\{\beta \geq 0\}} \int_{I}\left(\lambda_{\beta, n}^{1}(z)-\lambda_{\beta}(z)\right) \mathbb{1}\left\{z \in P_{n}(H)\right\} \mathrm{d} z+\int_{I_{2, n}} \lambda_{\beta}(z) \mathrm{d} z \\
= & (-1)^{\mathbb{1}\{\beta \geq 0\}} \int_{I}\left(\lambda_{\beta, n}^{1}(z)-\lambda_{\beta, n}^{0}(z)\right) \mathrm{d} z+\int_{I_{2, n}} \lambda_{\beta}(z) \mathrm{d} z .
\end{aligned}
$$


In view of the first assertion stated in Lemma 5.6, we can write

$$
\begin{aligned}
& \int_{I}\left|\lambda_{\beta, n}^{1}(z)-\lambda_{\beta}(z)\right| \mathrm{d} z \\
= & (-1)^{\mathbb{1}\{\beta \geq 0\}}\left(\int_{I} \lambda_{\beta, n}^{1}(z) \mathrm{d} z-\int_{I} \lambda_{\beta, n}^{0}(z) \mathrm{d} z\right)+\int_{I_{2, n}} \lambda_{\beta}(z) \mathrm{d} z . \\
= & (-1)^{\mathbb{1}\{\beta \geq 0\}}\left(\Lambda_{\beta, n}^{1}(I)-\Lambda_{\beta, n}^{0}(I)\right)+\int_{I} \lambda_{\beta}(z) \cdot \mathbb{1}\left\{z \in I_{2, n}\right\} \mathrm{d} z .
\end{aligned}
$$

By Lemma 5.6 we both have $\Lambda_{\beta, n}^{1}(I)-\Lambda_{\beta, n}^{0}(I) \rightarrow 0$ and $\Lambda_{\beta}(I)<\infty$. Since $m_{d}\left(I_{2, n}\right) \rightarrow$ 0 , the dominated convergence theorem shows that the integral figuring in (5.14) tends to 0 , too. Remembering (5.13), we have proven (5.12), and Scheffé's Lemma yields

$$
\lim _{n \rightarrow \infty} \Lambda_{\beta, n}^{1}(B)=\Lambda_{\beta}(B)
$$

Choosing $\varepsilon>0$ arbitrarily small, $\kappa_{n}(B) \rightarrow \Lambda_{\beta}(B)$ now follows from (5.11).

Remark 5.2. Notice that the redefinition of $\nu=1 /(d+1+2 \beta)$ induces the factor $1 / n$ in $(5.10)$. In the proof of a correspondingly adapted version of Lemma 4.8, this factor $1 / n$ and the factor $n$ cancel out again, as necessary for the convergence of the point processes of points lying close to the poles. See Remark 4.9 for some more details.

Since the connection between Lemma 5.1 - especially that of condition $(5.9)$ - and the setting given by Condition 5 is not completely obvious, we want to give some explanation: We write $\left.f\right|_{\ell}:=f \cdot \mathbb{1}\left\{z_{1}<0\right\}$. Remember that $P_{1}(H)$ results from the translation of the left half $\operatorname{int}(E) \cap\left\{z_{1}<0\right\}$ of $\operatorname{int}(E)$ to the right by $a_{1} \cdot \mathbf{e}_{1}$ along the $z_{1}$-axis (see the explanations preceeding (4.1)). This transformation is given by $T^{\ell}(z):=\left(z_{1}+a_{1}, \widetilde{z}\right)$. In doing so, the density $\left.z \mapsto f\right|_{\ell}(z)$ is transformed into $\left.z \mapsto f\right|_{\ell}\left(z_{1}-a_{1}, \widetilde{z}\right)$, and because of Condition 5 (see especially (5.2)) we get

$$
\left.f\right|_{\ell}\left(z_{1}-a_{1}, \widetilde{z}\right)=(1+o(1)) \cdot \alpha_{\ell}\left(1-\left(\frac{z_{1}-a_{1}}{a_{1}}\right)^{2}-\sum_{k=2}^{d}\left(\frac{z_{k}}{a_{k}}\right)^{2}\right)^{\beta_{\ell}}
$$

as $\delta \rightarrow 0$, with $o(1)$ uniformly on $P_{1}(H) \cap \mathbb{1}\left\{z_{1}<\delta\right\}$. We thus indeed apply Lemma 5.1. In the same way we write $\left.f\right|_{r}:=f \cdot \mathbb{1}\left\{z_{1}>0\right\}$. Here, the set $P_{1}(H)$ results from the translation of the right half $\operatorname{int}(E) \cap\left\{z_{1}>0\right\}$ of $\operatorname{int}(E)$ to the left by $-a_{1} \cdot \mathbf{e}_{1}$ along the $z_{1}$-axis and an additional reflection at the plane $\left\{z_{1}=0\right\}$ (see again the explanations preceeding (4.1)). This transformation is given by $T^{r}(z):=\left(-\left(z_{1}-a_{1}\right), \widetilde{z}\right)=\left(-z_{1}+a_{1}, \widetilde{z}\right)$. Applying $T^{r}$, the density $\left.z \mapsto f\right|_{r}(z)$ is 
transformed into $\left.z \mapsto f\right|_{r}\left(a_{1}-z_{1}, \widetilde{z}\right)$, and because of Condition 5 we obtain

$$
\left.f\right|_{r}\left(a_{1}-z_{1}, \widetilde{z}\right)=(1+o(1)) \cdot \alpha_{r}\left(1-\left(\frac{a_{1}-z_{1}}{a_{1}}\right)^{2}-\sum_{k=2}^{d}\left(\frac{z_{k}}{a_{k}}\right)^{2}\right)^{\beta_{r}}
$$

as $\delta \rightarrow 0$, with $o(1)$ uniformly on $P_{1}(H) \cap \mathbb{1}\left\{z_{1}<\delta\right\}$. Since $\left(a_{1}-z_{1}\right)^{2}=\left(z_{1}-a_{1}\right)^{2}$, we can apply Lemma 5.1 also in this case.

Now we can state the asymptotical behavior of $\operatorname{diam}\left(\mathbf{Z}_{n}\right)$ under Condition 5. Remember that $\Lambda_{\beta}$ had been defined in $(5.6)$ as the measure on $\mathbb{R}^{d}$ with Lebesgue density

$$
\lambda_{\beta}(z)=\left(\frac{2 z_{1}}{a_{1}}-\sum_{k=2}^{d}\left(\frac{z_{k}}{a_{k}}\right)^{2}\right)^{\beta} \mathbb{1}\{z \in P(H)\} .
$$

Theorem 5.3. Let the density $f$ be supported by the ellipsoid $E$ with half-axes $a_{1}>a_{2} \geq \ldots \geq a_{d}>0$ and satisfy Condition 5 with $\beta_{\ell}=\beta_{r}=: \beta$. We then have

$$
n^{\frac{2}{d+1+2 \beta}}\left(2 a_{1}-\operatorname{diam}\left(\mathbf{Z}_{n}\right)\right) \stackrel{\mathcal{D}}{\longrightarrow} \min _{i, j \geq 1}\left\{\mathcal{X}_{i, 1}+\mathcal{Y}_{j, 1}-\frac{1}{4 a_{1}}\left|\tilde{\mathcal{X}}_{i}-\widetilde{\mathcal{Y}}_{j}\right|^{2}\right\},
$$

where $\left\{\mathcal{X}_{i}, i \geq 1\right\} \stackrel{\mathcal{D}}{=} \operatorname{PRM}\left(\alpha_{\ell} \cdot \Lambda_{\beta}\right)$ and $\left\{\mathcal{Y}_{j}, j \geq 1\right\} \stackrel{\mathcal{D}}{=} \operatorname{PRM}\left(\alpha_{r} \cdot \Lambda_{\beta}\right)$ are independent Poisson processes. If Condition 5 and - without loss of generality - the inequality $\beta_{\ell}>\beta_{r}$ hold true, we obtain

$$
n^{\frac{2}{d+1+2 \beta_{\ell}}}\left(2 a_{1}-\operatorname{diam}\left(\mathbf{Z}_{n}\right)\right) \stackrel{\mathcal{D}}{\longrightarrow} \min _{i \geq 1}\left\{\mathcal{X}_{i, 1}-\frac{1}{4 a_{1}}\left|\widetilde{\mathcal{X}}_{i}\right|^{2}\right\}
$$

with $\left\{\mathcal{X}_{i}, i \geq 1\right\} \stackrel{\mathcal{D}}{=} \operatorname{PRM}\left(\alpha_{\ell} \cdot \Lambda_{\beta_{\ell}}\right)$. The same results hold true if we replace $\operatorname{diam}\left(\mathbf{Z}_{n}\right)$ with $M_{n}$.

Proof. Under Condition 5 we have $f(z)>0$ for each $z$ arbitrarily close to one of the poles. In the case $\beta_{\ell}=\beta_{r}$, this inequality allows us to copy the proof of Theorem 3.5 almost completely. The only difference is that we have to apply Lemma 5.1 instead of Lemma 4.6 to show an adapted version of Lemma 4.8. In the case $\beta_{\ell}>\beta_{r}$ we will observe a higher magnitude of points lying close to the right pole than to the left. This higher magnitude has far-reaching implications for the proof to follow. First of all, we define

$$
\nu_{\ell}:=\frac{1}{d+1+2 \beta_{\ell}}
$$




$$
T_{n}^{\ell}(z):=\left(n^{2 \nu_{\ell}} z_{1}, n^{\nu_{\ell}} \widetilde{z}\right)
$$

and $P_{n}^{\ell}(H):=T_{n}^{\ell}\left(P_{1}(H)\right)$. The beginning of the main part of the proof of Theorem 3.5 in Section 4.3 can be copied in this case, too. We will only point out the differences. Let $N_{r, n}, X_{1}, X_{2}, \ldots$ and $Y_{1}, Y_{2}, \ldots$ be defined as in the proof of Theorem 3.5 and write $V^{r}:=\left(a-Y_{1,1}, \widetilde{Y}_{1}\right)$. Then,

$$
\widetilde{\mathbf{Y}}_{n}:=\sum_{j=1}^{N_{r, n}} \varepsilon_{\left(a-Y_{j, 1}, \widetilde{Y}_{j}\right)}
$$

is a Poisson process with intensity measure $n c_{r, \delta} \cdot \mathbb{P}_{V^{r}}$, and $\mathbf{Y}_{n}^{\ell}:=\widetilde{\mathbf{Y}}_{n} \circ\left(T_{n}^{\ell}\right)^{-1}$ - taking the part of $\mathbf{Y}_{n}$ in the proof of Theorem 3.5 - is a Poisson process with intensity measure $\widehat{\mu}_{n}:=n c_{r, \delta} \cdot \mathbb{P}_{V^{r}} \circ\left(T_{n}^{\ell}\right)^{-1}$. The density $f$ fulfills Condition 5 at the right pole with power $\beta_{r}$, but the shifted process $\widetilde{\mathbf{Y}}_{n}$ is scaled via $T_{n}^{\ell}$, which depends on $\beta_{\ell}$, not on $\beta_{r}$. Broadly speaking, this 'wrong' (too slow) scaling has the effect, that $\mathbf{Y}_{n}^{\ell}$ will generate more and more points arbitrarily close to $\mathbf{0}$, and it will hence not converge in distribution toward a limiting Poisson process. We need to specify this behavior in the following: Since the density $f$ fulfills Condition 5 at the right pole with respect to $\alpha_{r}$ and $\beta_{r}$, the random vector $V^{r}$ fulfills condition (5.9) of Lemma 5.1, with $\alpha$ replaced with $\alpha_{r} / c_{r, \delta}$ and $\beta$ replaced with $\beta_{r}$ (remember the construction of $Y_{1}$ in the proof of Theorem 3.5). Then, in the proof of Lemma 5.1, we have

$$
\begin{gathered}
\mathbb{P}\left(T_{n}^{\ell}\left(V^{r}\right) \in B\right)=\frac{1}{n^{(d+1) \nu_{\ell}}} \int_{B} \widehat{g}\left(\frac{z_{1}}{n^{2 \nu_{\ell}}}, \frac{1}{n^{\nu_{\ell}}} \widetilde{z}\right)\left(1-\left(\frac{\frac{z_{1}}{n^{2 \nu_{\ell}}}-a_{1}}{a_{1}}\right)^{2}-\sum_{k=2}^{d}\left(\frac{\frac{z_{k}}{n^{\nu_{\ell}}}}{a_{k}}\right)^{2}\right)^{\beta_{r}} \\
\cdot \frac{\alpha_{r}}{c_{r, \delta}} \cdot \mathbb{1}\left\{z \in P_{n}^{\ell}(H)\right\} \mathrm{d} z .
\end{gathered}
$$

Using the abbreviation $J_{n}^{\ell}(z):=\widehat{g}\left(\frac{z_{1}}{n^{2 \nu_{\ell}}}, \frac{1}{n^{\nu} \ell} \widetilde{z}\right) \cdot \mathbb{1}\left\{z \in P_{n}^{\ell}(H)\right\}$ yields

$$
\begin{aligned}
& \mathbb{P}\left(T_{n}^{\ell}\left(V^{r}\right) \in B\right) \\
= & \frac{\alpha_{r}}{c_{r, \delta}} \cdot \frac{1}{n^{(d+1) \nu_{\ell}}} \cdot \int_{B}\left(1-\left(\frac{z_{1}}{n^{2 \nu_{\ell}} a_{1}}\right)^{2}+\frac{2 z_{1}}{n^{2 \nu_{\ell}} a_{1}}-1-\sum_{k=2}^{d}\left(\frac{z_{k}}{n^{\nu \ell} a_{k}}\right)^{2}\right)^{\beta_{r}} J_{n}^{\ell}(z) \mathrm{d} z \\
= & \frac{\alpha_{r}}{c_{r, \delta}} \cdot \frac{1}{n^{(d+1) \nu_{\ell}}} \cdot \frac{1}{n^{2 \beta_{r} \nu_{\ell}}} \int_{B}\left(\frac{2 z_{1}}{a_{1}}-\left(\frac{z_{1}}{n^{\nu_{\ell}} a_{1}}\right)^{2}-\sum_{k=2}^{d}\left(\frac{z_{k}}{a_{k}}\right)^{2}\right)^{\beta_{r}} J_{n}^{\ell}(z) \mathrm{d} z \\
= & \frac{\alpha_{r}}{c_{r, \delta}} \cdot \frac{1}{n^{\left(d+1+2 \beta_{\ell}\right) \nu_{\ell}}} \cdot \frac{1}{n^{-2 \nu_{\ell}\left(\beta_{\ell}-\beta_{r}\right)}} \cdot \int_{B} \widehat{g}\left(\frac{z_{1}}{n^{2 \nu_{\ell}}}, \frac{1}{n^{\nu_{\ell}}} \widetilde{z}\right) \cdot \lambda_{\beta_{r}, n}^{1}(z) \mathrm{d} z,
\end{aligned}
$$

if we use the definition of $\lambda_{\beta, n}^{1}$ given in 5.5 with respect to $\nu_{\ell}$ instead of $\nu$. Observe 
that this modification corresponds with the definition of $P_{n}^{\ell}(H)$, figuring in $J_{n}^{\ell}(z)$. So, the only dependence of $\lambda_{\beta_{r}, n}^{1}$ on $\beta_{r}$ is given by the power $\beta_{r}$, the support $P_{n}^{\ell}(H)$ of this density does only depend on $\beta_{\ell}$, not on $\beta_{r}$. Writing

$$
\widehat{\kappa}_{n}(B):=\int_{B} \widehat{g}\left(\frac{z_{1}}{n^{2 \nu_{\ell}}}, \frac{1}{n^{\nu_{\ell}}} \widetilde{z}\right) \cdot \lambda_{\beta_{r}, n}^{1}(z) \mathrm{d} z
$$

for $B \in \mathcal{B}^{d}$ and observing $\left(d+1+2 \beta_{\ell}\right) \nu_{\ell}=1$, we obtain

$$
\mathbb{P}\left(T_{n}^{\ell}\left(V^{r}\right) \in B\right)=\frac{\alpha_{r}}{c_{r, \delta}} \cdot \frac{1}{n} \cdot \widehat{\kappa}_{n}(B) \cdot n^{2 \nu_{\ell}\left(\beta_{\ell}-\beta_{r}\right)}
$$

In the same way as seen in the proof of Lemma 5.1, we get $\widehat{\kappa}_{n}(B) \rightarrow \Lambda_{\beta_{r}}(B)$. For $I:=\left\{z_{1} \leq \varepsilon\right\}$ with $\varepsilon>0$, we especially infer

$$
\begin{aligned}
\widehat{\mu}_{n}(I) & =n c_{r, \delta} \cdot\left(\mathbb{P}_{V^{r}} \circ\left(T_{n}^{\ell}\right)^{-1}\right)(I) \\
& =n c_{r, \delta} \cdot \mathbb{P}\left(T_{n}^{\ell}\left(V^{r}\right) \in I\right) \\
& =\alpha_{r} \cdot \widehat{\kappa}_{n}(I) \cdot n^{2 \nu_{\ell}\left(\beta_{\ell}-\beta_{r}\right)},
\end{aligned}
$$

and since $\beta_{\ell}>\beta_{r}$ and $\widehat{\kappa}_{n}(I) \rightarrow \Lambda_{\beta_{r}}(I)>0$, we see $\widehat{\mu}_{n}(I) \rightarrow \infty$, for each $\varepsilon>0$. Since $\widehat{\mu}_{n}$ is the intensity measure of the Poisson process $\mathbf{Y}_{n}^{\ell}$ and the support of $\Lambda_{\beta_{r}}$ is $P(H) \subset\left\{z_{1} \geq 0\right\}$, we can conclude that this process generates more and more points arbitrarily close to $\mathbf{0}$, formally

$$
\mathbf{Y}_{n}^{\ell}\left(P(H) \cap\left\{z_{1} \leq \varepsilon\right\}\right) \rightarrow \infty
$$

almost surely for each $\varepsilon>0$. As stated before, this process cannot converge in distribution. Observe that the limiting behavior of $\mathbf{X}_{n}^{\ell}:=\widetilde{\mathbf{X}}_{n} \circ\left(T_{n}^{\ell}\right)^{-1}$ - taking the part of $\mathbf{X}_{n}$ in the proof of Theorem 3.5 - does not change compared to the case $\beta_{\ell}=\beta_{r}$ : Since the density $f$ fulfills Condition 5 at the left pole with respect to $\alpha_{\ell}$ and $\beta_{\ell}$ and since the scaling function $T_{n}^{\ell}$ is defined in terms of $\beta_{\ell}$, we still have

$$
\mathbf{X}_{n}^{\ell}=\widetilde{\mathbf{X}}_{n} \circ\left(T_{n}^{\ell}\right)^{-1} \stackrel{\mathcal{D}}{\longrightarrow} \mathbf{X} \stackrel{\mathcal{D}}{=} \operatorname{PRM}\left(\alpha_{\ell} \cdot \Lambda_{\beta_{\ell}}\right)
$$

Using Lemma 5.6, given in Subsection 5.1.3, this weak convergence especially yields

$$
\mathbb{P}\left(\mathbf{X}_{n}^{\ell}\left(\left\{z_{1} \leq t\right\}\right)=0\right) \rightarrow \exp \left(-\alpha_{\ell} \cdot c_{\beta_{\ell}} \cdot t^{\frac{2 \beta_{\ell}+d+1}{2}}\right)
$$

where $t>0$. Remembering $\beta_{\ell}>-1$ shows $2 \beta_{\ell}+d+1>0$ and that the probability of observing at least one point of $\mathbf{X}_{n}^{\ell}$ left of $\left\{z_{1}=t\right\}$ is getting arbitrarily close to 1 
(at least for sufficiently large $n$ ) if we choose $t$ large enough.

Looking at the proof of Theorem 3.5, we now have to investigate the asymptotical behavior of $t_{1}\left(\widehat{G}\left(\mathbf{X}_{n}^{\ell} \times \mathbf{Y}_{n}^{\ell}\right)\right)$. Since $\mathbf{Y}_{n}^{\ell}$ does no longer converge in distribution, we cannot apply the continuous mapping theorem as seen in the proof of Theorem 3.5 in this setting.

In the proof of Lemma 4.10 we have seen that

$$
\frac{1-\eta}{2}\left(x_{1}+y_{1}\right) \leq G(x, y) \leq x_{1}+y_{1}
$$

for all $x, y \in \widehat{\eta} \cdot P(H)$ and $0<\frac{1-\eta}{2}<\frac{1}{2}$ (remember $H=H_{\ell}=H_{r}$ in this section). These inequalities can be interpreted as follows: If $x_{1}$ and/or $y_{1}$ is 'large', $G(x, y)$ has to be 'large', too, and if both $x_{1}$ and $y_{1}$ are 'small', then $G(x, y)$ has to be 'small', too. Broadly speaking, we can say that

$$
t_{1}\left(\widehat{G}\left(\mathbf{X}_{n}^{\ell} \times \mathbf{Y}_{n}^{\ell}\right)\right)=\min _{(i, j) \in I_{n}}\left\{G\left(n^{2 \nu_{\ell}}\left(a+X_{i, 1}\right), n^{\nu_{\ell}} \widetilde{X}_{i}, n^{2 \nu_{\ell}}\left(a-Y_{j, 1}\right), n^{\nu_{\ell}} \widetilde{Y}_{j}\right)\right\}
$$

will be determined by two points $\left(n^{2 \nu_{\ell}}\left(a+X_{i, 1}\right), n^{\nu_{\ell}} \widetilde{X}_{i}\right)$ and $\left(n^{2 \nu_{\ell}}\left(a-Y_{j, 1}\right), n^{\nu_{\ell}} \widetilde{Y}_{j}\right)$ with 'small' $z_{1}$-components. For being more precise, we define for $t, \varepsilon \geq 0$ the set

$$
A_{t, \varepsilon}:=\left(\widehat{\eta} \cdot P(H) \cap\left\{z_{1} \leq t\right\}\right) \times\left(\widehat{\eta} \cdot P(H) \cap\left\{z_{1} \leq \varepsilon\right\}\right)
$$

From (5.17) and the different asymptotical behavior of $\mathbf{X}_{n}^{\ell}$ and $\mathbf{Y}_{n}^{\ell}$ described above, we know that for each $\delta>0$ there is some $K>0$ so that

$$
\mathbb{P}\left(t_{1}\left(\widehat{G}\left(\left.\left(\mathbf{X}_{n}^{\ell} \times \mathbf{Y}_{n}^{\ell}\right)\right|_{A_{K, \varepsilon}}\right)\right)=t_{1}\left(\widehat{G}\left(\mathbf{X}_{n}^{\ell} \times \mathbf{Y}_{n}^{\ell}\right)\right)\right) \geq 1-\delta
$$

for any $\varepsilon>0$ and each sufficiently large $n$. Observe that the event figuring in (5.18) is nothing but the event that

$$
\min _{(i, j) \in I_{n}}\left\{G\left(n^{2 \nu_{\ell}}\left(a+X_{i, 1}\right), n^{\nu_{\ell}} \widetilde{X}_{i}, n^{2 \nu_{\ell}}\left(a-Y_{j, 1}\right), n^{\nu_{\ell}} \widetilde{Y}_{j}\right)\right\}
$$

is attained by a point $\left(n^{2 \nu_{\ell}}\left(a+X_{i, 1}\right), n^{\nu_{\ell}} \widetilde{X}_{i}, n^{2 \nu_{\ell}}\left(a-Y_{j, 1}\right), n^{\nu_{\ell}} \widetilde{Y}_{j}\right) \in A_{K, \varepsilon}$, i.e. by a point $X_{i}$ with $n^{2 \nu_{\ell}}\left(a+X_{i, 1}\right) \leq K$ and a point $Y_{j}$ with $n^{2 \nu_{\ell}}\left(a-Y_{j, 1}\right) \leq \varepsilon$. Define now

$$
G^{*}(x):=G(x, \mathbf{0})=x_{1}-\frac{1}{4 a_{1}}|\widetilde{x}|^{2} .
$$

The basic idea in the following is to choose $\varepsilon$ - depending on $K$, and hence depending 
on $\delta$ - small enough, to obtain

$$
G^{*}(x)-\delta \leq G(x, y) \leq G^{*}(x)+\delta
$$

for each $(x, y) \in A_{K, \varepsilon}$. Observe that we use the same $\delta$ as in (5.18). Later, we will consider $\delta \rightarrow 0$. Then, the probability figuring in (5.18) will tend to 1 and the difference between $G^{*}(x)$ and $G(x, y)$ on the set $A_{K, \varepsilon}$ will become negligible simultaneously. To this end, observe that

$$
\begin{aligned}
G(x, y) & =x_{1}+y_{1}-\frac{1}{4 a_{1}}|\widetilde{x}-\widetilde{y}|^{2} \\
& =x_{1}+y_{1}-\frac{1}{4 a_{1}}|\widetilde{x}|^{2}+\frac{1}{2 a_{1}} \widetilde{x}^{\top} \widetilde{y}-\frac{1}{4 a_{1}}|\widetilde{y}|^{2} \\
& =G^{*}(x)+R^{*}(x, y)
\end{aligned}
$$

with

$$
R^{*}(x, y):=y_{1}+\frac{1}{2 a_{1}} \widetilde{x}^{\top} \widetilde{y}-\frac{1}{4 a_{1}}|\widetilde{y}|^{2}
$$

We now want to find bounds for $R^{*}(x, y)$, that depend solely on $x_{1}$ and $y_{1}$. Since $\left|\widetilde{x}^{\top} \widetilde{y}\right| \leq|\widetilde{x}| \cdot|\widetilde{y}|$, we can focus on finding an upper bound for $|\widetilde{z}|$ on $\widehat{\eta} \cdot P(H)$. In view of Remark 3.6 and the proof of Lemma 3.12, we can choose $\eta$ in such a way that

$$
\frac{1}{\kappa_{2}^{\ell}}+\frac{1}{\kappa_{2}^{r}}=2 a_{1} \eta \quad \Longleftrightarrow \quad 2 \frac{a_{2}^{2}}{a_{1}}=2 a_{1} \eta \quad \Longleftrightarrow \quad \eta=\left(\frac{a_{2}}{a_{1}}\right)^{2}
$$

and hence

$$
\widehat{\eta}=\frac{1+\eta^{-1}}{2}=\frac{1+\left(\frac{a_{1}}{a_{2}}\right)^{2}}{2}=\frac{a_{1}^{2}+a_{2}^{2}}{2 a_{2}^{2}} .
$$

For each $z \in \widehat{\eta} \cdot P(H)$, Remark 4.2, Remark 3.6 and $a_{2} \geq \ldots \geq a_{d}$ imply

$$
\frac{1}{2} \sum_{k=2}^{d} \frac{a_{1}}{a_{k}^{2}} \cdot z_{k}^{2} \leq \frac{a_{1}^{2}+a_{2}^{2}}{2 a_{2}^{2}} \cdot z_{1} \quad \Longrightarrow \quad \frac{1}{a_{2}^{2}} \sum_{k=2}^{d} z_{k}^{2} \leq \frac{a_{1}^{2}+a_{2}^{2}}{a_{1} a_{2}^{2}} \cdot z_{1} \quad \Longrightarrow \quad|\widetilde{z}| \leq c \cdot \sqrt{z_{1}}
$$

with

$$
c:=\sqrt{\frac{a_{1}^{2}+a_{2}^{2}}{a_{1}}}
$$

Hence,

$$
-c^{2} \cdot \sqrt{K \cdot \varepsilon} \leq \widetilde{x}^{\top} \widetilde{y} \leq c^{2} \cdot \sqrt{K \cdot \varepsilon}
$$


and

$$
0-\frac{c^{2}}{2 a_{1}} \cdot \sqrt{K \cdot \varepsilon}-\frac{c^{2}}{4 a_{1}} \cdot \varepsilon \leq R^{*}(x, y) \leq \varepsilon+\frac{c^{2}}{2 a_{1}} \cdot \sqrt{K \cdot \varepsilon}-0
$$

for each $(x, y) \in A_{K, \varepsilon}$. Given $\delta>0$, the constant $K>0$ had been chosen fixed. But, since $(5.18)$ holds true for any $\varepsilon>0$, we can choose $\varepsilon>0$ small enough to obtain

$$
-\delta \leq R^{*}(x, y) \leq \delta
$$

and we get

$$
G^{*}(x)-\delta \leq G(x, y) \leq G^{*}(x)+\delta
$$

for each $(x, y) \in A_{K, \varepsilon}$. Similar to the proof of Theorem 3.5, we define the function

$$
\widehat{G}^{*}:\left\{\begin{array}{l}
M_{p}(\widehat{\eta} \cdot P(H)) \rightarrow M_{p}\left(\mathbb{R}_{+}\right) \\
\mu \mapsto \mu \circ\left(G^{*}\right)^{-1}
\end{array}\right.
$$

Since $G^{*}(x)=G(x, \mathbf{0})$, the proof of Lemma 4.10 shows that the function $\widehat{G}^{*}$ is continuous, too. Hence, $(\overline{5.16})$ and the continuous mapping theorem yield

$$
\widehat{G}^{*}\left(\mathbf{X}_{n}^{\ell}\right) \stackrel{\mathcal{D}}{\longrightarrow} \widehat{G}^{*}(\mathbf{X})
$$

and in the same way as seen in the proof of Theorem 3.5 we deduce that

$$
t_{1}\left(\widehat{G}^{*}\left(\mathbf{X}_{n}^{\ell}\right)\right) \stackrel{\mathcal{D}}{\longrightarrow} t_{1}\left(\widehat{G}^{*}(\mathbf{X})\right)=\min _{i \geq 1}\left\{\mathcal{X}_{i, 1}-\frac{1}{4 a_{1}}\left|\widetilde{\mathcal{X}}_{i}\right|^{2}\right\}
$$

Letting $\delta \rightarrow 0$, this convergence, together with (5.19), implies

$$
t_{1}\left(\widehat{G}\left(\mathbf{X}_{n}^{\ell} \times \mathbf{Y}_{n}^{\ell}\right)\right) \stackrel{\mathcal{D}}{\longrightarrow} \min _{i \geq 1}\left\{\mathcal{X}_{i, 1}-\frac{1}{4 a_{1}}\left|\widetilde{\mathcal{X}}_{i}\right|^{2}\right\}
$$

and the proof is finished.

\subsubsection{Application to Pearson Type II distributions}

Example 5.4. We now consider the so-called $d$-dimensional symmetric multivariate Pearson Type II distributions supported by an ellipsoid with half-axes $a_{1}>a_{2} \geq$ $\ldots \geq a_{d}>0$, where $d \geq 2$. According to equation (2.43) in Fang et al. [8] and Example 2.11 in the same reference, we know that the corresponding densities are given by

$$
f_{\beta}(z)=f_{1}^{\beta}\left(z^{\top} \Sigma^{-1} z\right) \cdot \mathbb{1}\{z \in \operatorname{int}(E)\}
$$


with

$$
f_{1}^{\beta}(t)=\operatorname{det}(\Sigma)^{-\frac{1}{2}} \cdot \frac{\Gamma\left(\frac{d}{2}+\beta+1\right)}{\Gamma(\beta+1) \pi^{\frac{d}{2}}}(1-t)^{\beta},
$$

for $0 \leq t<1$ and $\beta>-1$. Remembering $\Sigma=\operatorname{diag}\left(a_{1}^{2}, \ldots, a_{d}^{2}\right)$, we obtain

$$
f_{\beta}(z)=\frac{\Gamma\left(\frac{d}{2}+\beta+1\right)}{\Gamma(\beta+1) \pi^{\frac{d}{2}} \prod_{i=1}^{d} a_{i}}\left(1-z^{\top} \Sigma^{-1} z\right)^{\beta} \cdot \mathbb{1}\{z \in \operatorname{int}(E)\}
$$

Hence, Condition 5 holds true with $\beta_{\ell}=\beta_{r}=\beta$ and

$$
\alpha:=\alpha_{\ell}=\alpha_{r}=\frac{\Gamma\left(\frac{d}{2}+\beta+1\right)}{\Gamma(\beta+1) \pi^{\frac{d}{2}} \prod_{i=1}^{d} a_{i}},
$$

so that we can apply Theorem 5.3.

Figures 5.4, 5.6, 5.8 and 5.10 illustrate the densities $f_{\beta}$ and the corresponding densities of the intensity measures $\alpha \cdot \Lambda_{\beta}$ in the setting of Example 5.4 for $d=2, a_{1}=$ $1, a_{2}=1 / 2$ and $\beta \in\{-1 / 2,0,1,2\}$. The results of a simulation study in each of these cases are displayed in Figures 5.5, 5.7, 5.9 and 5.11. As in the simulation presented after Corollary 3.7, the limiting distributions have been approximated by simulating the limiting processes $\left\{\mathcal{X}_{i}, i \geq 1\right\}$ and $\left\{\mathcal{Y}_{i}, i \geq 1\right\}$ only on $P(H) \cap\left\{z \in \mathbb{R}^{2}: z_{1} \leq b\right\}$ for some $b>0$, not on the whole limiting set $P(H)$ itself. To obtain a good approximation with moderate computing effort it is necessary to choose $b$ subject to $\beta$. Simulations exhibit that a reasonable choice of $b$ is given by the solution of the equation

$$
\alpha \cdot \Lambda_{\beta}\left(\left\{z_{1} \leq b\right\}\right)=10 .
$$

We thus choose $b$ in such a way that the numbers of points of the approximating processes follow two independent Poisson distributions with parameter 10. In view of Lemma 5.6, we have $\Lambda_{\beta}\left(\left\{z_{1} \leq b\right\}\right)=c_{\beta} \cdot b^{\frac{2 \beta+d+1}{2}}$, with $c_{\beta}$ given in the same lemma. Some calculations show that $b=20$ if $\beta=-1 / 2$, and for $\beta=0,1,2$ the approximative values of $b$ are $6.52,2.55$ and 1.67 , respectively. So, the larger we choose $\beta$, the smaller the set $P(H) \cap\left\{z \in \mathbb{R}^{2}: z_{1} \leq b\right\}$ becomes. As another implication of a larger value of $\beta$ we observe a smaller quantity of points of the process $\mathbf{Z}_{n}$, that realize close to the poles of $E$. This lack of points close to the poles induces a slower rate of convergence in Theorem 5.3 with increasing $\beta$. For $\beta \in\{-1 / 2,0\}$ it was sufficient to choose $n=1000$ to obtain a good match between the empirical distribution functions of $n^{2 /(3+2 \beta)}\left(2 a_{1}-\operatorname{diam}\left(\mathbf{Z}_{n}\right)\right)$ and those of the approximated limiting distributions, see Figures 5.5 and 5.7. For $\beta=1$ we had to choose $n=10000$, and for $\beta=2$ it was already necessary to take $n=100000$ for keeping the differences between the 
empirical distribution functions small, see Figures 5.9 and 5.11 .
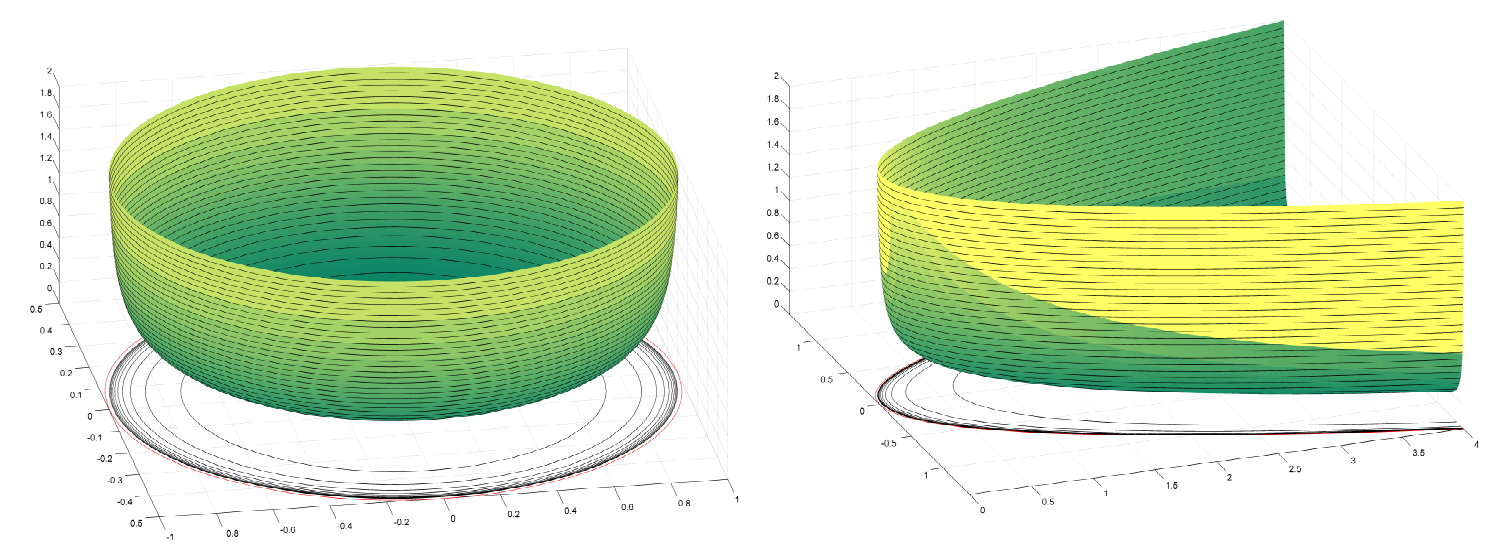

Figure 5.4: The density $f_{\beta}$ (left) and that of the intensity measure $\alpha \cdot \Lambda_{\beta}$ (right) in the setting of Example 5.4 for $d=2$ with $a_{1}=1, a_{2}=1 / 2$ and $\beta=-1 / 2$.

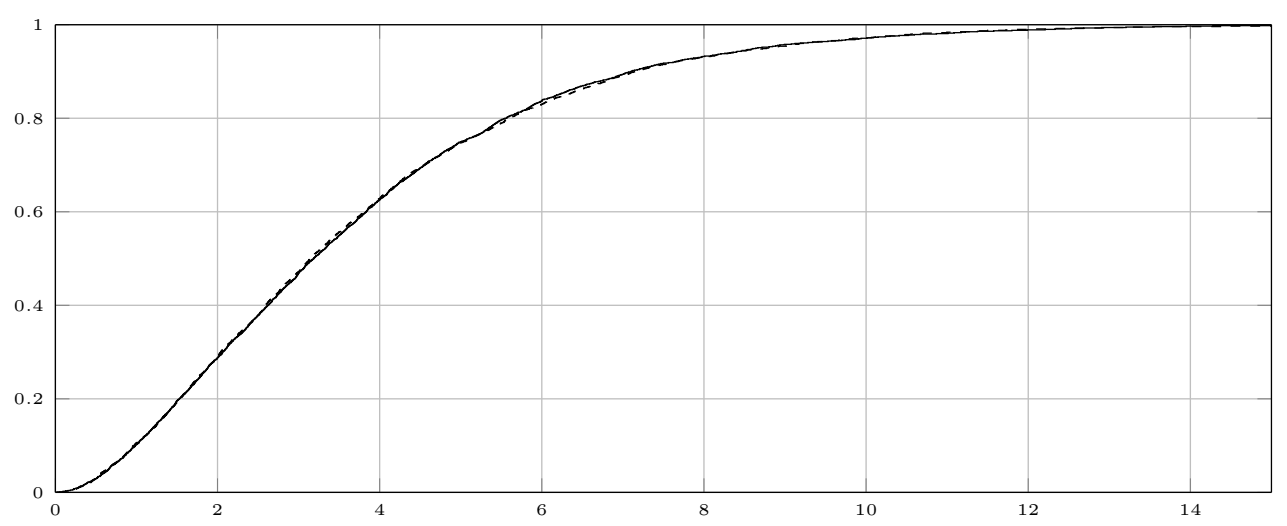

Figure 5.5: Empirical distribution function of $n\left(2-M_{n}\right)$ in the setting of Example 5.4 for $d=2$ with $a_{1}=1, a_{2}=1 / 2, \beta=-1 / 2$ and $n=1000$ (solid, 5000 replications). The limit distribution is approximated as described after Example 5.4 with $b=20$ (dashed, 5000 replications). 

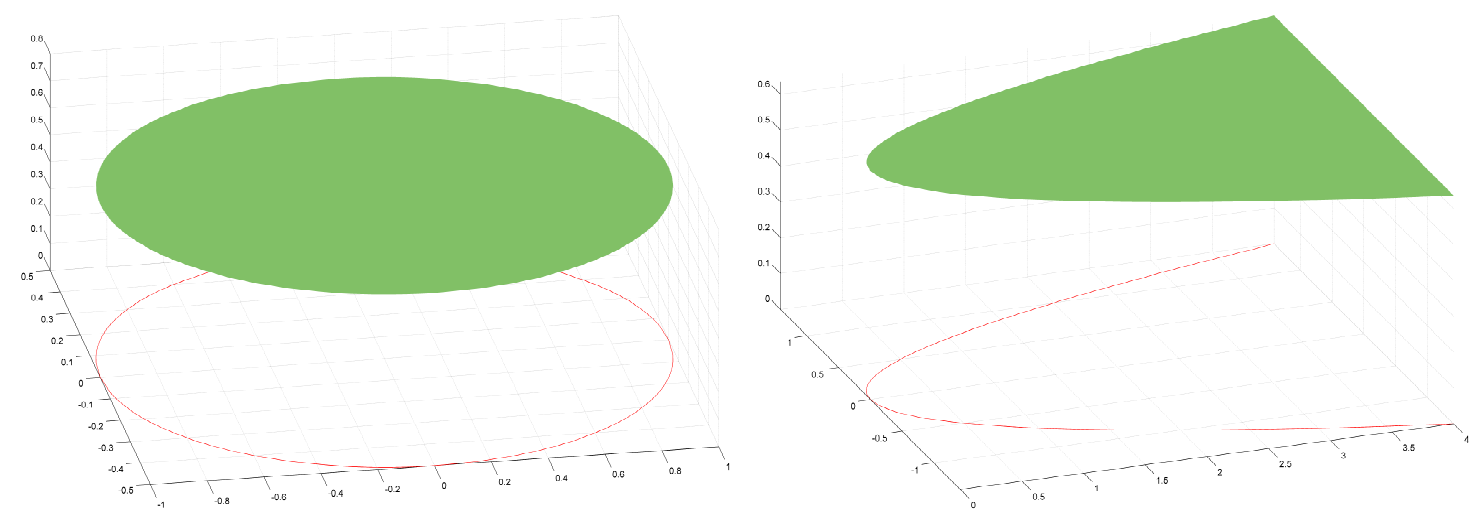

Figure 5.6: The density $f_{\beta}$ (left) and that of the intensity measure $\alpha \cdot \Lambda_{\beta}$ (right) in the setting of Example 5.4 for $d=2$ with $a_{1}=1, a_{2}=1 / 2$ and $\beta=0$.

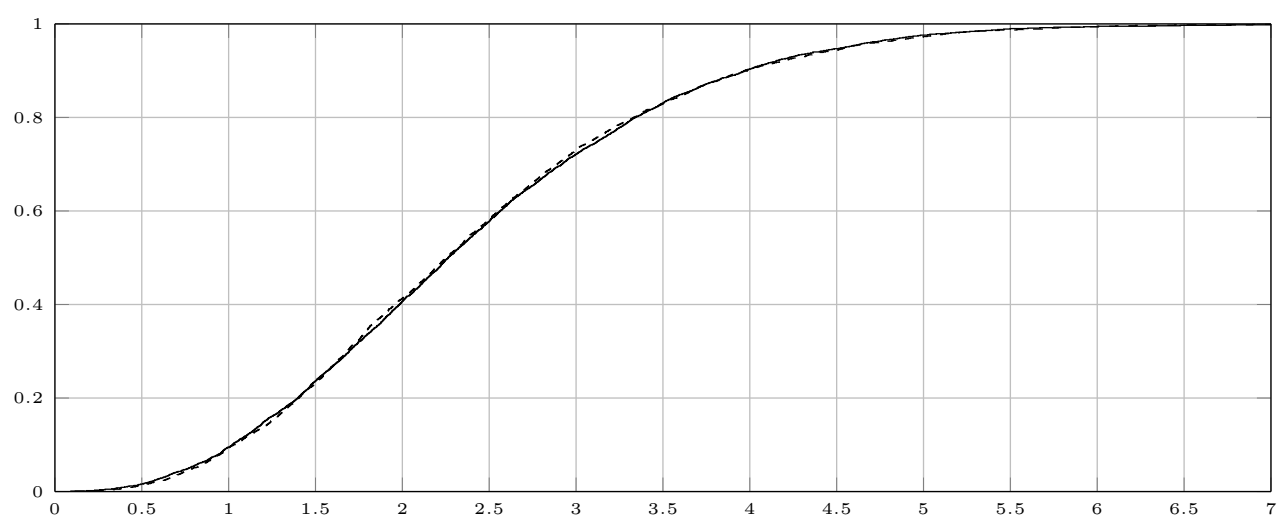

Figure 5.7: Empirical distribution function of $n^{2 / 3}\left(2-M_{n}\right)$ in the setting of Example 5.4 for $d=2$ with $a_{1}=1, a_{2}=1 / 2, \beta=0$ and $n=1000$ (solid, 5000 replications). The limit distribution is approximated as described after Example 5.4 with $b \approx 6.52$ (dashed, 5000 replications).
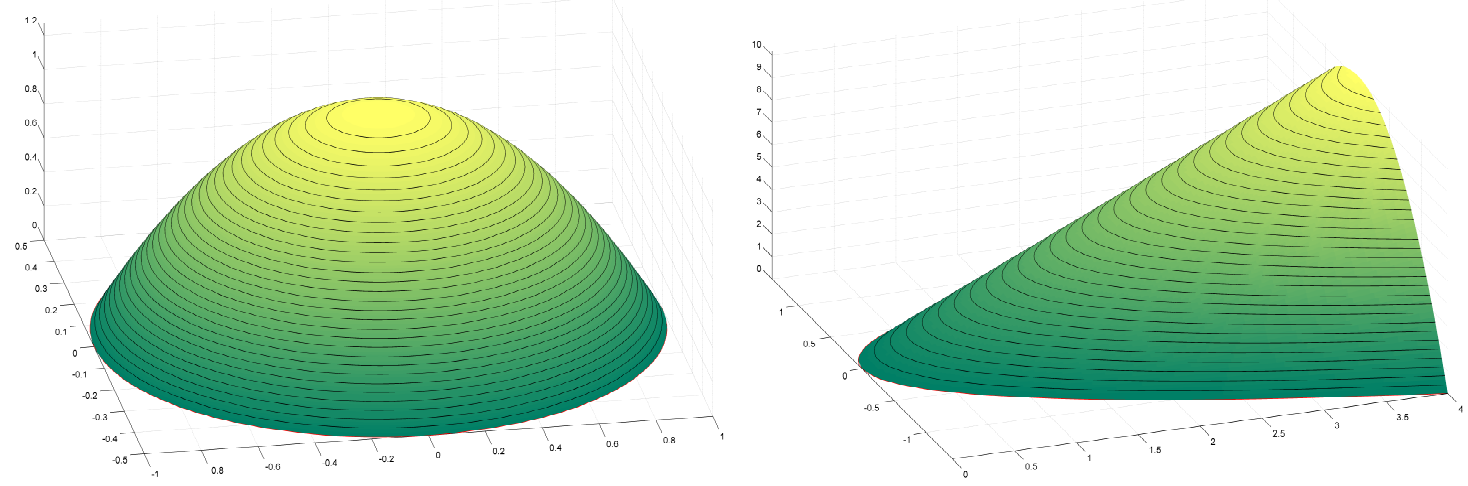

Figure 5.8: The density $f_{\beta}$ (left) and that of the intensity measure $\alpha \cdot \Lambda_{\beta}$ (right) in the setting of Example 5.4 for $d=2$ with $a_{1}=1, a_{2}=1 / 2$ and $\beta=1$. 


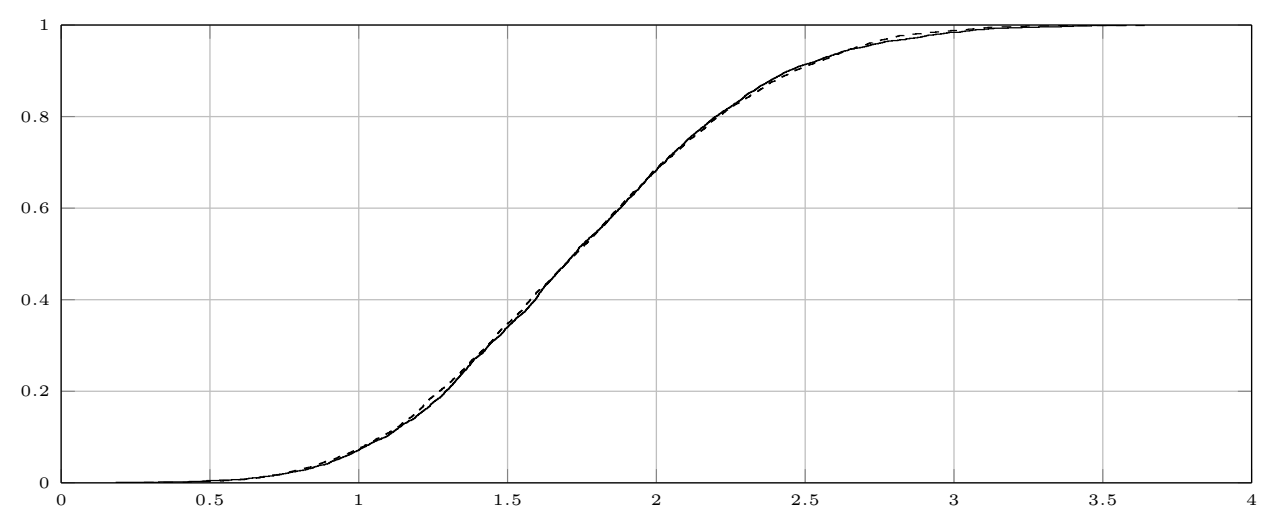

Figure 5.9: Empirical distribution function of $n^{2 / 5}\left(2-M_{n}\right)$ in the setting of Example 5.4 for $d=2$ with $a_{1}=1, a_{2}=1 / 2, \beta=1$ and $n=10000$ (solid, 5000 replications). The limit distribution is approximated as described after Example 5.4 with $b \approx 2.55$ (dashed, 5000 replications).
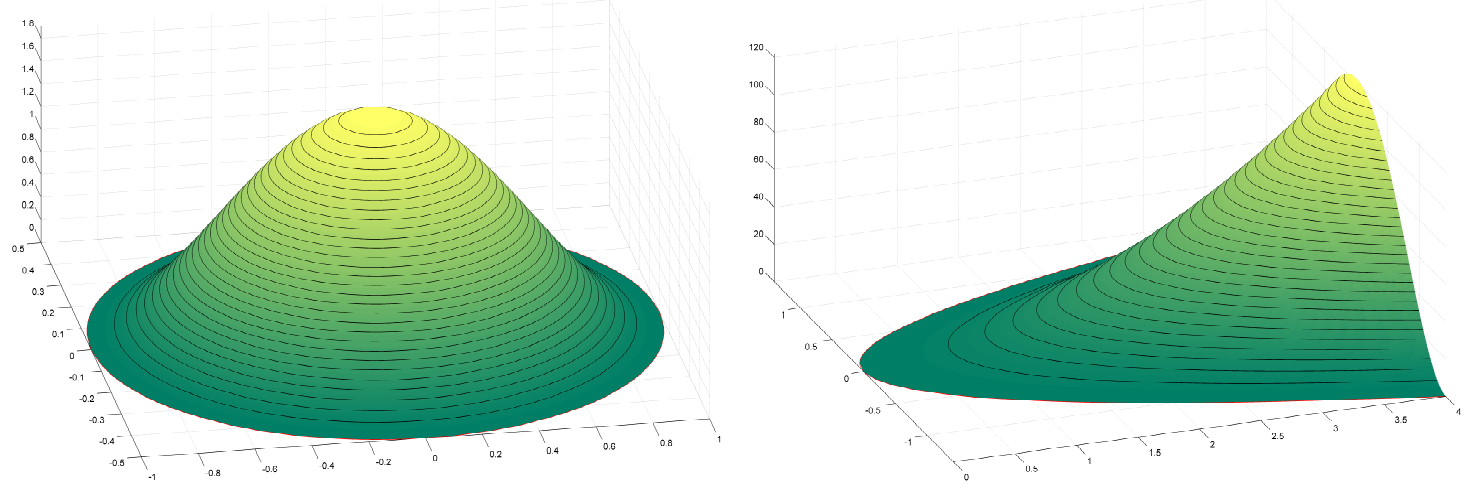

Figure 5.10: The density $f_{\beta}$ (left) and that of the intensity measure $\alpha \cdot \Lambda_{\beta}$ (right) in the setting of Example 5.4 for $d=2$ with $a_{1}=1, a_{2}=1 / 2$ and $\beta=2$.

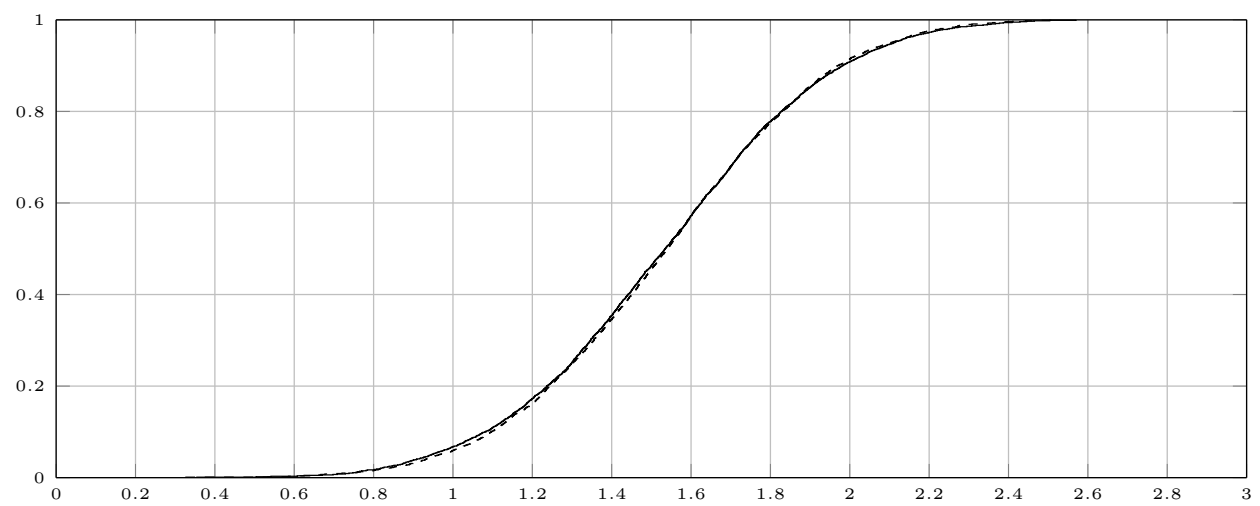

Figure 5.11: Empirical distribution function of $n^{2 / 7}\left(2-M_{n}\right)$ in the setting of Example 5.4 for $d=2$ with $a_{1}=1, a_{2}=1 / 2, \beta=2$ and $n=100000$ (solid, 5000 replications). The limit distribution is approximated as described after Example 5.4 with $b \approx 1.67$ (dashed, 5000 replications). 
Example 5.5. In generalization of Example 5.4 we now consider the 'combination' of two Pearson Type II distributions. Being more precise, we take $\beta_{\ell}>\beta_{r}>-1$ and look at the piecewise defined density

$$
\begin{aligned}
f(z)= & \frac{\Gamma\left(\frac{d}{2}+\beta_{\ell}+1\right)}{\Gamma\left(\beta_{\ell}+1\right) \pi^{\frac{d}{2}} \prod_{i=1}^{d} a_{i}}\left(1-z^{\top} \Sigma^{-1} z\right)^{\beta_{\ell}} \mathbb{1}\left\{z \in \operatorname{int}(E), z_{1}<0\right\} \\
+ & \frac{\Gamma\left(\frac{d}{2}+\beta_{r}+1\right)}{\Gamma\left(\beta_{r}+1\right) \pi^{\frac{d}{2}} \prod_{i=1}^{d} a_{i}}\left(1-z^{\top} \Sigma^{-1} z\right)^{\beta_{r}} \mathbb{1}\left\{z \in \operatorname{int}(E), 0<z_{1}\right\} .
\end{aligned}
$$

In this case we can apply the second assertion of Theorem 5.3 and obtain

$$
n^{\frac{2}{d+1+2 \beta_{\ell}}}\left(2 a_{1}-\operatorname{diam}\left(\mathbf{Z}_{n}\right)\right) \stackrel{\mathcal{D}}{\longrightarrow} \min _{i \geq 1}\left\{\mathcal{X}_{i, 1}-\frac{1}{4 a_{1}}\left|\widetilde{\mathcal{X}}_{i}\right|^{2}\right\}
$$

with $\left\{\mathcal{X}_{i}, i \geq 1\right\} \stackrel{\mathcal{D}}{=} \operatorname{PRM}\left(\alpha_{\ell} \cdot \Lambda_{\beta_{\ell}}\right)$ and

$$
\alpha_{\ell}:=\frac{\Gamma\left(\frac{d}{2}+\beta_{\ell}+1\right)}{\Gamma\left(\beta_{\ell}+1\right) \pi^{\frac{d}{2}} \prod_{i=1}^{d} a_{i}} .
$$

Figure 5.12 shows 2500 random points in the setting of this example for $d=2$ with $a_{1}=1, a_{2}=1 / 2, \beta_{\ell}=1, \beta_{r}=-1 / 2$, and Figure 5.13 illustrates the result of a simulation study with the sample size $n=100000$.

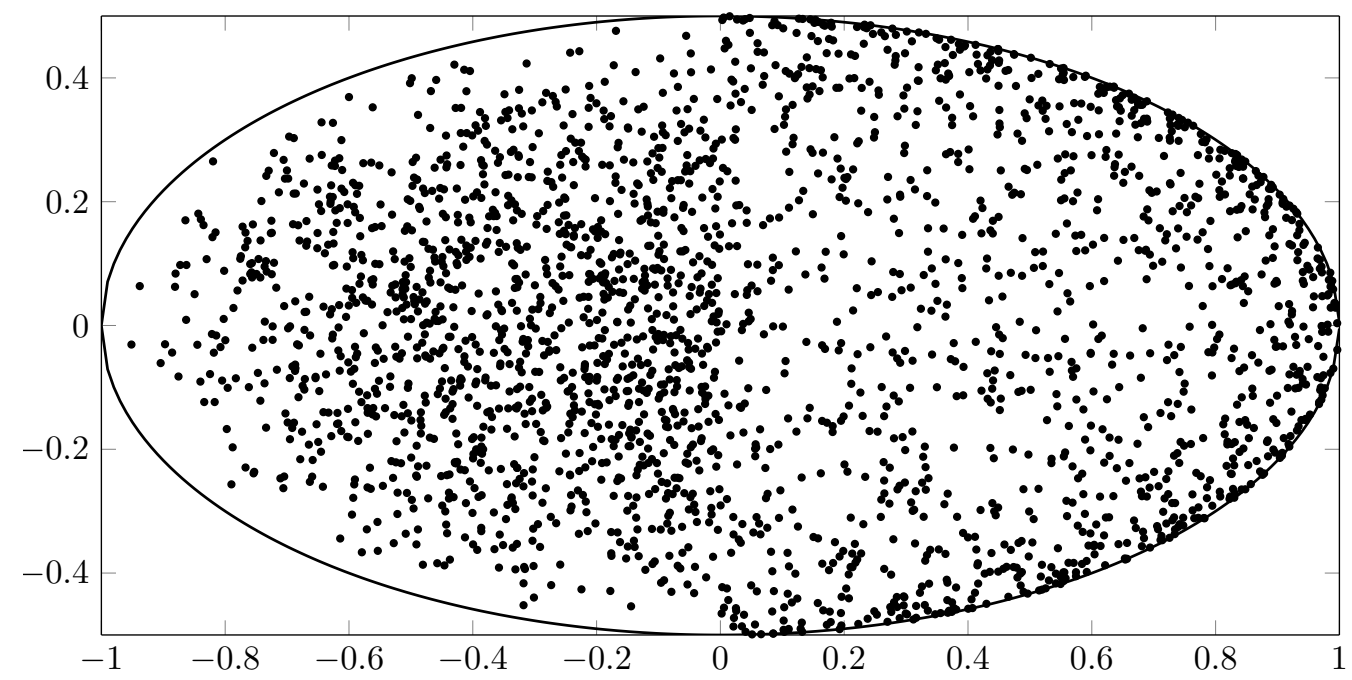

Figure 5.12: Simulation of 2500 random points in the setting of Example 5.5 for $d=2$ with $a_{1}=1, a_{2}=1 / 2, \beta_{\ell}=1$ and $\beta_{r}=-1 / 2$. 


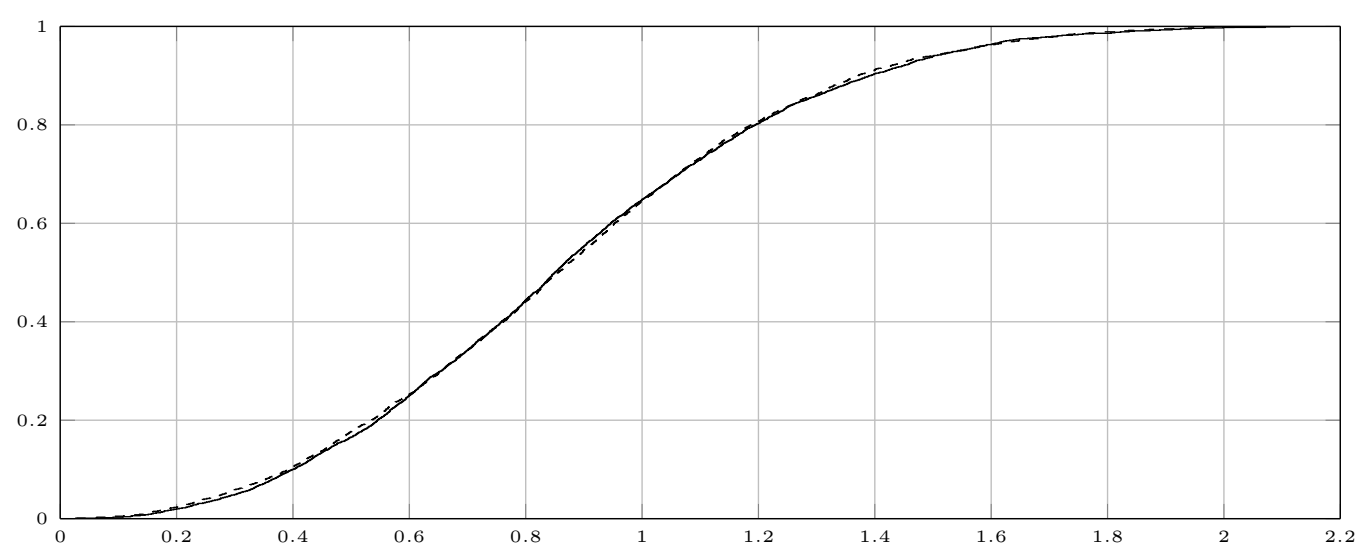

Figure 5.13: Empirical distribution function of $n^{2 / 5}\left(2-M_{n}\right)$ in the setting of Example 5.5 for $d=2$ with $a_{1}=1, a_{2}=1 / 2, \beta_{\ell}=1, \beta_{r}=-1 / 2$ and $n=100000$ (solid, 5000 replications). The limit distribution is approximated in the same way as described after Example 5.4 with $b \approx 2.55$ (dashed, 5000 replications).

\subsubsection{TeChnical Details For SubSection 5.1.1}

The following lemma has been an essential tool for the proof of Lemma 5.1. Since its proof is long and technical, two parts of it can be found as Lemma 5.7 and Lemma 5.8 after the main part of the proof.

Lemma 5.6. Let $t>0, I:=\left\{z_{1} \leq t\right\}$ and $i \in\{0,1\}$. We then have $\Lambda_{\beta, n}^{i}(I)<\infty$ for sufficiently large $n$, and

$$
\left|\Lambda_{\beta, n}^{1}(I)-\Lambda_{\beta, n}^{0}(I)\right| \rightarrow 0 \quad \text { as } n \rightarrow \infty .
$$

Furthermore,

$$
\Lambda_{\beta}(I)=c_{\beta} \cdot t^{\frac{2 \beta+d+1}{2}}
$$

where

$$
c_{\beta}:=\left(\frac{2}{a_{1}}\right)^{\frac{2 \beta+d-1}{2}} \cdot \frac{(d-1) \cdot \omega_{d-1}}{2 \beta+d+1} \cdot B\left(\beta+1, \frac{d-1}{2}\right) \cdot \prod_{k=2}^{d} a_{k} .
$$

Proof. Since the calculations are lengthy, we calculate $\Lambda_{\beta, n}^{i}(I)$ simultaneously for $i \in\{0,1\}$. As the density $\lambda_{\beta, n}^{i}$ of $\Lambda_{\beta, n}^{i}$ is supported by $P_{n}(H) \subset\left\{z_{1} \geq 0\right\}$ for each $n \in \mathbb{N}$, only the set $\left\{0 \leq z_{1} \leq t\right\}$ has to be considered. We choose $n_{0} \in \mathbb{N}$ subject to

$$
t<n_{0}^{2 \nu} a_{1}
$$


This inequality especially implies

$$
\frac{2 z_{1}}{a_{1}}-\left(\frac{z_{1}}{n^{\nu} a_{1}}\right)^{2}>0
$$

for each $z_{1} \in(0, t)$ and each $n \geq n_{0}$, and we will only consider $n \geq n_{0}$ in the following. For fixed $z_{1} \in(0, t)$, and without stressing the dependence on $z_{1}$, we define

$$
b_{k, n}^{i}:=a_{k} \sqrt{\frac{2 z_{1}}{a_{1}}-i \cdot\left(\frac{z_{1}}{n^{\nu} a_{1}}\right)^{2}},
$$

where $k \in\{2, \ldots, d\}$. Remembering the representation of $P_{n}(H)$ given in (5.3), we see that

$$
\begin{aligned}
S_{n}\left(z_{1}\right): & =\left\{\widetilde{z} \in \mathbb{R}^{d-1}:\left(z_{1}, \widetilde{z}\right) \in P_{n}(H)\right\} \\
& =\left\{\widetilde{z} \in \mathbb{R}^{d-1}: \sum_{k=2}^{d}\left(\frac{z_{k}}{a_{k}}\right)^{2}<\frac{2 z_{1}}{a_{1}}-\left(\frac{z_{1}}{n^{\nu} a_{1}}\right)^{2}\right\} \\
& =\left\{\widetilde{z} \in \mathbb{R}^{d-1}: \sum_{k=2}^{d}\left(\frac{z_{k}}{b_{k, n}^{1}}\right)^{2}<1\right\}
\end{aligned}
$$

is a $(d-1)$-dimensional ellipsoid with half-axes $b_{2, n}^{1}, \ldots, b_{d, n}^{1}$. Using the special form of $I$ and Cavalieri's principle, we obtain

$$
\begin{aligned}
\Lambda_{\beta, n}^{i}(I) & =\int_{I}\left(\frac{2 z_{1}}{a_{1}}-i \cdot\left(\frac{z_{1}}{n^{\nu} a_{1}}\right)^{2}-\sum_{k=2}^{d}\left(\frac{z_{k}}{a_{k}}\right)^{2}\right)^{\beta} \mathbb{1}\left\{z \in P_{n}(H)\right\} \mathrm{d} z \\
& =\int_{0}^{t} \int_{S_{n}\left(z_{1}\right)}\left(\frac{2 z_{1}}{a_{1}}-i \cdot\left(\frac{z_{1}}{n^{\nu} a_{1}}\right)^{2}-\sum_{k=2}^{d}\left(\frac{z_{k}}{a_{k}}\right)^{2}\right)^{\beta} \mathrm{d} \widetilde{z} \mathrm{~d} z_{1} \\
& =\int_{0}^{t}\left(\frac{2 z_{1}}{a_{1}}-i \cdot\left(\frac{z_{1}}{n^{\nu} a_{1}}\right)^{2}\right)^{\beta} \int_{S_{n}\left(z_{1}\right)}\left(1-\sum_{k=2}^{d}\left(\frac{z_{k}}{b_{k, n}^{i}}\right)^{2}\right)^{\beta} \mathrm{d} \widetilde{z} \mathrm{~d} z_{1} .
\end{aligned}
$$

Putting $\widehat{T}_{i}(\widetilde{z}):=\left(z_{2} / b_{2, n}^{i}, \ldots, z_{d} / b_{d, n}^{i}\right)$, we get $\widehat{T}_{i}^{-1}(\widetilde{y})=\left(b_{2, n}^{i} \cdot y_{2}, \ldots, b_{d, n}^{i} \cdot y_{d}\right)$ and

$$
\Delta \widehat{T}_{i}^{-1}(\widetilde{y})=\operatorname{det}\left(\operatorname{diag}\left(b_{2, n}^{i}, \ldots, b_{d, n}^{i}\right)\right)=\left(\frac{2 z_{1}}{a_{1}}-i \cdot\left(\frac{z_{1}}{n^{\nu} a_{1}}\right)^{2}\right)^{\frac{d-1}{2}} \prod_{k=2}^{d} a_{k}
$$


Since

$$
\frac{b_{k, n}^{i}}{b_{k, n}^{1}}=\frac{\sqrt{\frac{2 z_{1}}{a_{1}}-i \cdot\left(\frac{z_{1}}{n^{\nu} a_{1}}\right)^{2}}}{\sqrt{\frac{2 z_{1}}{a_{1}}-\left(\frac{z_{1}}{n^{\nu} a_{1}}\right)^{2}}}=\frac{\sqrt{1-i \cdot \frac{z_{1}}{2 n^{2 \nu} a_{1}}}}{\sqrt{1-\frac{z_{1}}{2 n^{2 \nu} a_{1}}}}
$$

it follows that

$$
\begin{aligned}
\widehat{T}_{i}\left(S_{n}\left(z_{1}\right)\right) & =\left\{\widetilde{y} \in \mathbb{R}^{d-1}: \widehat{T}_{i}^{-1}(\widetilde{y}) \in S_{n}\left(z_{1}\right)\right\} \\
& =\left\{\widetilde{y} \in \mathbb{R}^{d-1}: \sum_{k=2}^{d}\left(\frac{b_{k, n}^{i} y_{k}}{b_{k, n}^{1}}\right)^{2}<1\right\} \\
& =\left\{\widetilde{y} \in \mathbb{R}^{d-1}: \sum_{k=2}^{d} y_{k}^{2}<\frac{1-\frac{z_{1}}{2 n^{2 \nu} a_{1}}}{1-i \cdot \frac{z_{1}}{2 n^{2 \nu} a_{1}}}\right\} .
\end{aligned}
$$

Notice that $\widehat{T}_{i}\left(S_{n}\left(z_{1}\right)\right)$ is the open $(d-1)$-dimensional ball with centre $\mathbf{0}$ and radius

$$
r_{n}^{i}\left(z_{1}\right):= \begin{cases}1, & i=1 \\ \sqrt{1-\frac{z_{1}}{2 n^{2 \nu} a_{1}},}, & i=0 .\end{cases}
$$

Since the boundary of this (open) ball has Lebesgue measure 0 , we can consider the closed ball $B_{r_{n}^{i}\left(z_{1}\right)}(\mathbf{0})$ instead of $\widehat{T}_{i}\left(S_{n}\left(z_{1}\right)\right)$ in the integrals to follow. Applying the transformation $\widehat{T}_{i}$ to the inner integral figuring in (5.21) yields

$$
\begin{aligned}
& \Lambda_{\beta, n}^{i}(I) \\
= & \int_{0}^{t}\left(\frac{2 z_{1}}{a_{1}}-i \cdot\left(\frac{z_{1}}{n^{\nu} a_{1}}\right)^{2}\right)^{\beta} \\
& \cdot \int_{B_{r_{n}^{i}\left(z_{1}\right)}(\mathbf{0})}\left(1-\sum_{k=2}^{d} y_{k}^{2}\right)^{\beta}\left(\frac{2 z_{1}}{a_{1}}-i \cdot\left(\frac{z_{1}}{n^{\nu} a_{1}}\right)^{2}\right)^{\frac{d-1}{2}}\left(\prod_{k=2}^{d} a_{k}\right) \mathrm{d} \widetilde{y} \mathrm{~d} z_{1} \\
= & \left(\prod_{k=2}^{d} a_{k}\right) \cdot \int_{0}^{t}\left(\frac{2 z_{1}}{a_{1}}-i \cdot\left(\frac{z_{1}}{n^{\nu} a_{1}}\right)^{2}\right)^{\beta+\frac{d-1}{2}} \cdot\left(\int_{B_{r_{n}^{i}\left(z_{1}\right)}(\mathbf{0})}\left(1-|\widetilde{y}|^{2}\right)^{\beta} \mathrm{d} \widetilde{y}\right) \mathrm{d} z_{1} \\
= & \left(\prod_{k=2}^{d} a_{k}\right) \cdot \int_{0}^{t} g_{n}^{i}\left(z_{1}\right) \cdot I_{n}^{i}\left(z_{1}\right) \mathrm{d} z_{1},
\end{aligned}
$$


where

$$
\begin{aligned}
g_{n}^{i}\left(z_{1}\right) & :=\left(\frac{2 z_{1}}{a_{1}}-i \cdot\left(\frac{z_{1}}{n^{\nu} a_{1}}\right)^{2}\right)^{\rho}, \\
\rho & :=\beta+\frac{d-1}{2}
\end{aligned}
$$

and

$$
I_{n}^{i}\left(z_{1}\right):=\int_{B_{r_{n}^{i}\left(z_{1}\right)}(\mathbf{0})}\left(1-|\widetilde{y}|^{2}\right)^{\beta} \mathrm{d} \widetilde{y}
$$

To prove the asymptotical behavior of $\Lambda_{\beta, n}^{i}(I)$, we want to apply the dominated convergence theorem to the integral figuring in $(\sqrt{5.22})$. For this purpose, we need an integrable upper bound for $g_{n}^{i}\left(z_{1}\right) \cdot I_{n}^{i}\left(z_{1}\right)$ on $(0, t)$. In a first step we use the fact that

$$
0<\frac{2 z_{1}}{a_{1}}-i \cdot\left(\frac{z_{1}}{n_{0}^{\nu} a_{1}}\right)^{2} \leq \frac{2 z_{1}}{a_{1}}-i \cdot\left(\frac{z_{1}}{n^{\nu} a_{1}}\right)^{2} \leq \frac{2 z_{1}}{a_{1}}
$$

for each fixed $z_{1} \in(0, t)$ and $n \geq n_{0}$, see the beginning of this proof for the definition of $n_{0}$. Writing

$$
g_{\infty}\left(z_{1}\right):=\lim _{n \rightarrow \infty} g_{n}^{i}\left(z_{1}\right)=\left(\frac{2 z_{1}}{a_{1}}\right)^{\rho},
$$

we obtain the inequality

$$
g_{n}^{i}\left(z_{1}\right) \leq \begin{cases}g_{\infty}\left(z_{1}\right), & \text { if } \rho \geq 0 \\ g_{n_{0}}^{i}\left(z_{1}\right), & \text { if } \rho<0\end{cases}
$$

which holds for each $z_{1} \in(0, t)$ and each $n \geq n_{0}$. Notice that in both cases we have equality if $i=0$. Lemma 5.8 will show that $\int_{0}^{t} g_{\infty}\left(z_{1}\right) \mathrm{d} z_{1}<\infty$ and $\int_{0}^{t} g_{n}^{i}\left(z_{1}\right) \mathrm{d} z_{1}<\infty$ for each $n \geq n_{0}$. In a second step, notice that $0 \leq r_{n}^{i}\left(z_{1}\right) \leq 1$ for each $z_{1} \in(0, t)$ and $n \geq n_{0}$. Furthermore, for each fixed $z_{1} \in(0, t)$ we have $r_{n}^{i}\left(z_{1}\right) \uparrow 1$ and hence $B_{r_{n}^{i}\left(z_{1}\right)}(\mathbf{0}) \uparrow B_{1}(\mathbf{0})$. This implies

$$
I_{n}^{i}\left(z_{1}\right) \uparrow \int_{B_{1}(\mathbf{0})}\left(1-|\widetilde{y}|^{2}\right)^{\beta} \mathrm{d} \widetilde{y}=: \sigma_{\beta}<\infty,
$$

see Lemma 5.7 for the calculation of $\sigma_{\beta}$. Putting both parts together demonstrates 
that

$$
g_{n}^{i}\left(z_{1}\right) \cdot I_{n}^{i}\left(z_{1}\right) \leq \begin{cases}g_{\infty}\left(z_{1}\right) \cdot \sigma_{\beta}, & \text { if } \rho \geq 0 \\ g_{n_{0}}^{i}\left(z_{1}\right) \cdot \sigma_{\beta}, & \text { if } \rho<0\end{cases}
$$

and that both upper bounds in the line above are integrable on $(0, t)$. We thus can apply the dominated convergence theorem, and invoking again Lemma 5.8 we obtain

$$
\begin{aligned}
\lim _{n \rightarrow \infty} \Lambda_{\beta, n}^{i}(I) & =\left(\prod_{k=2}^{d} a_{k}\right) \cdot \int_{0}^{t} \lim _{n \rightarrow \infty} g_{n}^{i}\left(z_{1}\right) \cdot I_{n}^{i}\left(z_{1}\right) \mathrm{d} z_{1} \\
& =\left(\prod_{k=2}^{d} a_{k}\right) \cdot \int_{0}^{t}\left(\lim _{n \rightarrow \infty} g_{n}^{i}\left(z_{1}\right)\right) \cdot\left(\lim _{n \rightarrow \infty} I_{n}^{i}\left(z_{1}\right)\right) \mathrm{d} z_{1} \\
& =\left(\prod_{k=2}^{d} a_{k}\right) \cdot \int_{0}^{t} g_{\infty}\left(z_{1}\right) \cdot \sigma_{\beta} \mathrm{d} z_{1} \\
& =\left(\prod_{k=2}^{d} a_{k}\right)\left(\frac{2}{a_{1}}\right)^{\rho} \frac{\sigma_{\beta}}{\rho+1} \cdot t^{\rho+1} .
\end{aligned}
$$

Firstly, this result shows that $\Lambda_{\beta, n}^{i}(I)$ is finite for sufficiently large $n$. Secondly, notice that the limiting value above does not depend on $i \in\{0,1\}$ and hence

$$
\left|\Lambda_{\beta, n}^{1}(I)-\Lambda_{\beta, n}^{0}(I)\right| \rightarrow 0
$$

The calculation of $\Lambda_{\beta}(I)$ can be done in a similar way. One has to chose $i=0$ and to replace throughout $P_{n}(H)$ with $P(H)$. This results in $\widehat{T}_{0}\left(S_{n}\left(z_{1}\right)\right)=\operatorname{int}\left(B_{1}(\mathbf{0})\right)$ and $I_{n}^{0}\left(z_{1}\right)=\sigma_{\beta}$, independently of $n$ and $z_{1}$. Since $\rho+1=\beta+\frac{d-1}{2}+1=\frac{2 \beta+d+1}{2}$, Lemma 5.7 finishes the proof.

For better readability, two parts of the proof of Lemma 5.6 have been postponed. The first one is given by the following lemma:

Lemma 5.7. If $\beta>-1$ we have

$$
\sigma_{\beta}:=\int_{B_{1}(\mathbf{0})}\left(1-|\widetilde{y}|^{2}\right)^{\beta} \mathrm{d} \widetilde{y}=\frac{(d-1) \cdot \omega_{d-1}}{2} \cdot B\left(\beta+1, \frac{d-1}{2}\right) .
$$

Proof. Using $(d-1)$-dimensional spherical coordinates yields

$$
\int_{B_{1}(\mathbf{0})}\left(1-|\widetilde{y}|^{2}\right)^{\beta} \mathrm{d} \widetilde{y}=\int_{\mathcal{S}^{d-2}} \int_{0}^{1}\left(1-r^{2}\right)^{\beta} r^{d-2} \mathrm{~d} r \mathcal{H}^{d-2}(\mathrm{~d} u),
$$


and by substituting $r^{2}=s$ we get $\mathrm{d} r=\frac{1}{2} s^{-\frac{1}{2}} \mathrm{~d} s$ and hence

$$
\int_{B_{1}(\mathbf{0})}\left(1-|\widetilde{y}|^{2}\right)^{\beta} \mathrm{d} \widetilde{y}=\int_{\mathcal{S}^{d-2}} 1 \mathcal{H}^{d-2}(\mathrm{~d} u) \int_{0}^{1}(1-s)^{\beta} s^{\frac{d-2}{2}} \frac{1}{2} s^{-\frac{1}{2}} \mathrm{~d} s .
$$

Since the surface area of $\mathcal{S}^{d-2}$ is $(d-1) \cdot \omega_{d-1}$, we obtain

$$
\begin{aligned}
\int_{B_{1}(\mathbf{0})}\left(1-|\widetilde{y}|^{2}\right)^{\beta} \mathrm{d} \widetilde{y} & =(d-1) \cdot \omega_{d-1} \cdot \frac{1}{2} \int_{0}^{1}(1-s)^{\beta} s^{\frac{d-3}{2}} \mathrm{~d} s \\
& =\frac{(d-1) \cdot \omega_{d-1}}{2} \cdot B\left(\beta+1, \frac{d-1}{2}\right) .
\end{aligned}
$$

Before we can show the second part postponed from the proof of Lemma 5.6, we have to introduce Gauss' hypergeometric function and the incomplete Beta function: For $a, b, c \in \mathbb{R}$ and $c \notin\{\ldots,-2,-1,0\}$, Gauss' hypergeometric function is defined by

$$
F(a, b, c \mid x):=\frac{\Gamma(c)}{\Gamma(a) \Gamma(b)} \sum_{n=0}^{\infty} \frac{\Gamma(a+n) \Gamma(b+n)}{\Gamma(c+n)} \cdot \frac{x^{n}}{n !}, \quad-1<x<1 .
$$

The radius of convergence of this series is 1, see Abramowitz and Stegun [1, p. 556]. For fixed $x \in[0,1]$, the incomplete Beta function is given by

$$
B_{x}(a, b):=\int_{0}^{x} t^{a-1}(1-t)^{b-1} \mathrm{~d} t
$$

$a, b>0$, and we have the very useful relation

$$
B_{x}(a, b)=\frac{x^{a}}{a} F(a, 1-b, a+1 \mid x)
$$

see Abramowitz and Stegun [1, p. 263]. Notice that $B_{1}(\cdot, \cdot)$ is the Beta function $B(\cdot, \cdot)$, as seen before.

Now we can present the last missing part for the proof of Lemma 5.6. For this purpose, remember the definition of $n_{0}$ given at the beginning of the proof of Lemma 5.6.

Lemma 5.8. In the setting of Lemma 5.6 we have for each $n \geq n_{0}$

$$
\int_{0}^{t} g_{\infty}\left(z_{1}\right) \mathrm{d} z_{1}=\int_{0}^{t} g_{n}^{0}\left(z_{1}\right) \mathrm{d} z_{1}=\left(\frac{2}{a_{1}}\right)^{\rho} \frac{t^{\rho+1}}{\rho+1}<\infty
$$


and

$$
\int_{0}^{t} g_{n}^{1}\left(z_{1}\right) \mathrm{d} z_{1}=\left(\frac{2}{a_{1}}\right)^{\rho} \frac{t^{\rho+1}}{\rho+1} \cdot F\left(\rho+1,-\rho, \rho+2 \mid \frac{t}{2 n^{2 \nu} a_{1}}\right)<\infty .
$$

Proof. Since $g_{\infty}\left(z_{1}\right)=g_{n}^{0}\left(z_{1}\right)=\left(\frac{2 z_{1}}{a_{1}}\right)^{\rho}$, the first assertion is clear. To show the second assertion, we put $c_{n}:=2 n^{2 \nu} a_{1}$ and obtain

$$
\begin{aligned}
\int_{0}^{t} g_{n}^{1}\left(z_{1}\right) \mathrm{d} z_{1} & =\int_{0}^{t}\left(\frac{2 z_{1}}{a_{1}}-\left(\frac{z_{1}}{n^{\nu} a_{1}}\right)^{2}\right)^{\rho} \mathrm{d} z_{1} \\
& =\left(\frac{2}{a_{1}}\right)^{\rho} \int_{0}^{t} z_{1}^{\rho}\left(1-\frac{z_{1}}{c_{n}}\right)^{\rho} \mathrm{d} z_{1} .
\end{aligned}
$$

Substituting $\frac{z_{1}}{c_{n}}=x$ yields $\mathrm{d} z_{1}=c_{n} \mathrm{~d} x$ and

$$
\begin{aligned}
\int_{0}^{t} g_{n}^{1}\left(z_{1}\right) \mathrm{d} z_{1} & =\left(\frac{2}{a_{1}}\right)^{\rho} \int_{0}^{\frac{t}{c_{n}}}\left(c_{n} x\right)^{\rho}(1-x)^{\rho} c_{n} \mathrm{~d} x \\
& =\left(\frac{2}{a_{1}}\right)^{\rho} c_{n}^{\rho+1} \int_{0}^{\frac{t}{c_{n}}} x^{\rho}(1-x)^{\rho} \mathrm{d} x .
\end{aligned}
$$

In view of $(5.20)$ and $n \geq n_{0}$, we know that $t / c_{n} \in(0,1)$. Hence, we can use (5.23) and (5.24) to deduce

$$
\begin{aligned}
\int_{0}^{t} g_{n}^{1}\left(z_{1}\right) \mathrm{d} z_{1} & =\left(\frac{2}{a_{1}}\right)^{\rho} c_{n}^{\rho+1} B_{\frac{t}{c_{n}}}(\rho+1, \rho+1) \\
& =\left(\frac{2}{a_{1}}\right)^{\rho} c_{n}^{\rho+1} \frac{\left(\frac{t}{c_{n}}\right)^{\rho+1}}{\rho+1} F\left(\rho+1,-\rho, \rho+2 \mid \frac{t}{c_{n}}\right) \\
& =\left(\frac{2}{a_{1}}\right)^{\rho} \frac{t^{\rho+1}}{\rho+1} F\left(\rho+1,-\rho, \rho+2 \mid \frac{t}{c_{n}}\right) \\
& <\infty
\end{aligned}
$$

since the radius of convergence of Gauss' hypergeometric function $F(\rho+1,-\rho, \rho+2 \mid \cdot)$ is 1 .

\subsection{More General Densities Supported By General SETS}

\subsubsection{General CONSIDERATIONS}

In Subsection 5.1.1, we considered ellipsoids $E$ and densities of the form

$$
f(z)=\alpha \cdot\left(1-z^{\top} \Sigma^{-1} z\right)^{\beta} \cdot \mathbb{1}\{z \in \operatorname{int}(E)\},
$$


where $\beta>-1, \alpha>0$ and $\Sigma \in \mathbb{R}^{d \times d}$ depends on the half-axes of $E$. If we want to consider general densities on any set $E_{0}$ covered by Conditions 1 to 3 , we have to be very careful. To simplify matters, we now assume that the underlying set $E_{0}$ is symmetric with respect to the plane $\left\{z_{1}=0\right\}$, has a diameter of length $2 a>0$, and that the principal curvature directions at both poles are given by $\mathbf{e}_{2}, \ldots, \mathbf{e}_{d}$. Based on the given principal curvatures $\kappa_{2}, \ldots, \kappa_{d}$ of $E_{0}$ at the poles, we define $a_{k}:=\sqrt{\frac{a}{\kappa_{k}}}$ for $k \in\{2, \ldots, d\}$. In view of the calculations seen in Remark 3.6, the ellipsoid

$$
E:=\left\{z \in \mathbb{R}^{d}:\left(\frac{z_{1}}{a}\right)^{2}+\sum_{k=2}^{d}\left(\frac{z_{k}}{a_{k}}\right)^{2} \leq 1\right\}
$$

approximates the set $E_{0}$ at the poles, in the sense that the principal curvatures and the corresponding directions at the poles coincide. Putting $\Sigma_{0}:=\operatorname{diag}\left(a^{2}, a_{2}^{2}, \ldots, a_{d}^{2}\right)$, we can write $E=\left\{z \in \mathbb{R}^{d}: z^{\top} \Sigma_{0}^{-1} z \leq 1\right\}$, and we consider

$$
f_{0}(z)=\alpha \cdot\left(1-z^{\top} \Sigma_{0}^{-1} z\right)^{\beta} \cdot \mathbb{1}\left\{z \in \operatorname{int}\left(E_{0}\right)\right\}
$$

where $\beta>-1$ and $\alpha>0$. If $E_{0}$ is an ellipsoid and we choose $\alpha$ appropriately, the function $f_{0}$ is exactly the density of a Pearson Type II distribution. If $E_{0} \subset E$, and if we adjust the constant $\alpha$ appropriately, $f_{0}$ is a probability density, too. In this case, the assertion of Theorem 5.3 still holds true, since the support of the intensity measure $\Lambda_{\beta}$ of the limiting processes does not depend on whether we consider the set $E_{0}$ or the ellipsoid $E$. The reason for this coincidence lies in the very special choice of $E$ : Since the principal curvatures and directions of $E_{0}$ and $E$ at the poles are exactly the same, the corresponding osculating paraboloids (the support of $\Lambda_{\beta}$ ) also coincide. But, if we have $m_{d}\left(E_{0} \backslash E\right)>0$, the function $f_{0}$ takes negative (or even non-real) values and hence is no density. If the set $E_{0} \backslash E$ is contained in $\left\{-a+\delta \leq z_{1} \leq a-\delta\right\}$ for some $\delta>0$, we can redefine $f_{0}$ on the set $\left\{-a+\delta \leq z_{1} \leq a-\delta\right\}$ appropriately to obtain a probability density and then apply the same result as before, since the limit distribution of $M_{n}$ is only determined be the shape of $f_{0}$ close to the poles. But if, without loss of generality, we have $m_{d}\left(E_{0} \backslash E \cap\left\{z_{1} \leq-a+\delta\right\}\right)>0$ for each $\delta>0$, the definition of $f_{0}$ in 5.25$)$ is completely inappropriate to obtain a probability density supported by $E_{0}$. Nevertheless, it is possible to establish results similar to Theorem 5.3 for general densities supported by $E_{0}$. The crucial difference occurs in (the proof of) Lemma 5.1: Writing $g_{0}(z):=f_{0}\left(z_{1}-a_{1}, \widetilde{z}\right)$ for some probability density $f_{0}$ supported by $E_{0}$ and

$$
T_{n}^{0}(z):=\left(n^{2 \nu_{0}} z_{1}, n^{\nu_{0}} \widetilde{z}\right)
$$


with $\nu_{0}>0$ chosen suitably, we need that $g_{0}\left(\left(T_{n}^{0}\right)^{-1}(z)\right)$ converges towards a nondegenerate limit density. See Remark 5.2 for some comments on the correct choice of $\nu_{0}$ in the special case of Pearson Type II distributions. Instead of investigating this problem in complete generality, we consider an easy special case, which is given by densities that depend only on the $z_{1}$-component close to the poles, see the following subsection.

\subsubsection{A SPECIAL CLASS OF DENSITIES ON GENERAL SETS}

Let $E$ be a set with a diameter of length $2 a>0$, fulfilling Conditions 1 to 3 , and suppose $f$ satisfies the subsequent generalized version of Condition 4 :

Condition 6. We assume $f: E \rightarrow \mathbb{R}_{+}, \int_{E} f(z) \mathrm{d} z=1$ and that there are constants $\alpha_{\ell}, \alpha_{r}>0$ and $\beta_{\ell}, \beta_{r}>-\frac{d+1}{2}$ so that for $i \in\{\ell, r\}$, the function

$$
z \mapsto \frac{f(z)}{\alpha_{i}\left(a-\left|z_{1}\right|\right)^{\beta_{i}}}
$$

that maps from $E^{*}:=E \backslash\{(-a, \mathbf{0}),(a, \mathbf{0})\}$ into $\mathbb{R}_{+}$, can be extended continuously at the poles $(-a, \mathbf{0})$ and $(a, \mathbf{0})$ with value 1 . Thereby, $\alpha_{\ell}, \beta_{\ell}$ correspond to the left pole $(-a, \mathbf{0})$ and $\alpha_{r}, \beta_{r}$ to the right pole $(-a, \mathbf{0})$, respectively.

In Subsection 5.2.3 we will show that the choice $\beta_{\ell}, \beta_{r}>-\frac{d+1}{2}$ is appropriate for making $f$ integrable (close to the poles).

As before, we assume that $Z_{1}, Z_{2}, \ldots$ are i.i.d. with common density $f$. Now, we sketch the proof of a result that is very similar to Lemma 5.1 for the left pole of $E$. Since, in contrast to the situation in Subsection 5.1.1, the set $E$ is no longer assumed to be symmetric with respect to the plane $\left\{z_{1}=0\right\}$, we again have to write $H_{\ell}$ instead of $H$ for the Hessian of the boundary function $s^{\ell}$ at the left pole. As before, we have to consider the set $P_{1}\left(H_{\ell}\right)$, see the beginning of Section 4.1 for the (original) construction. Using the same construction for $E^{*}$ instead of $E$ and calling the resulting set $P_{1}^{*}\left(H_{\ell}\right)$, we obtain $P_{1}^{*}\left(H_{\ell}\right)=P_{1}\left(H_{\ell}\right) \backslash\{\mathbf{0}\}$, where $P_{1}\left(H_{\ell}\right)$ is the set defined at the beginning of Section 4.1 for the original set $E$.

For $0<z_{1}<a$, we have $a-\left|z_{1}-a\right|=a+z_{1}-a=z_{1}$, and defining $g(z):=f\left(z_{1}-a, \widetilde{z}\right)$, Condition 6 yields the equality $g(z)=(1+o(1)) \cdot \alpha_{\ell} \cdot z_{1}^{\beta_{\ell}}$, with $o(1)$ uniformly on $P_{1}^{*}\left(H_{\ell}\right) \cap\left\{z_{1} \leq \delta\right\}$ as $\delta \rightarrow 0$. Putting $\nu:=\left(d+1+2 \beta_{\ell}\right)^{-1}$ and $P_{n}^{*}\left(H_{\ell}\right):=T_{n}\left(P_{1}^{*}\left(H_{\ell}\right)\right)$, 
we obtain (cf. the proof of Lemma 5.1)

$$
\begin{aligned}
\mathbb{P}\left(T_{n}(V) \in B\right) & =\frac{1}{n^{(d+1) \nu}} \int_{B} g\left(\frac{z_{1}}{n^{2 \nu}}, \frac{1}{n^{\nu}} \widetilde{z}\right) \mathbb{1}\left\{z \in P_{n}^{*}\left(H_{\ell}\right)\right\} \mathrm{d} z \\
& =\frac{1}{n^{(d+1) \nu}} \int_{B} \alpha_{\ell}\left(\frac{z_{1}}{n^{2 \nu}}\right)^{\beta_{\ell}}(1+o(1)) \mathbb{1}\left\{z \in P_{n}^{*}\left(H_{\ell}\right)\right\} \mathrm{d} z \\
& =\frac{1}{n^{\left(d+1+2 \beta_{\ell}\right) \nu}} \cdot \alpha_{\ell} \int_{B} z_{1}^{\beta_{\ell}}(1+o(1)) \mathbb{1}\left\{z \in P_{n}^{*}\left(H_{\ell}\right)\right\} \mathrm{d} z \\
& =: \frac{1}{n} \cdot \alpha_{\ell} \cdot \kappa_{n}^{\ell}(B) .
\end{aligned}
$$

Using similar arguments as seen in the (long and technical) proof of Lemma 5.1 yields $\kappa_{n}^{\ell}(B) \rightarrow \Lambda_{\beta_{\ell}}^{*}(B)$, where

$$
\Lambda_{\beta_{i}}^{*}(B):=\int_{B} z_{1}^{\beta_{i}} \mathbb{1}\left\{z \in P^{*}\left(H_{i}\right)\right\} \mathrm{d} z
$$

$P^{*}\left(H_{i}\right):=P\left(H_{i}\right) \backslash\{\mathbf{0}\}, i \in\{\ell, r\}$, and $P\left(H_{i}\right)$ is defined as in (3.12). Notice that the crucial point for this convergence to hold true is $\beta_{\ell}>-\frac{d+1}{2}$. Under this condition, we have $\kappa_{n}^{\ell}(B)<\infty$ and $\Lambda_{\beta_{\ell}}^{*}(B)<\infty$, for each bounded Borel set $B \subset \mathbb{R}^{d}$ and sufficiently large $n$. This assertion is an immediate consequence of the integrability of $f$ close to the poles under Condition 6, proven in the following Subsection 5.2.3. Putting $g(z):=f\left(a-z_{1}, \widetilde{z}\right)$, a symmetry argument gives the same result for the right pole, if we throughout replace $\ell$ with $r$. Using exactly the same reasoning as in the proof of Theorem 5.3, we get the following result:

Theorem 5.9. Let $E$ be a set that satisfies Conditions 1 to 3 , and let $f$ be a density supported by $E$, satisfying Condition 6 with $\beta_{\ell}=\beta_{r}=: \beta$. We then have

$$
n^{\frac{2}{d+1+2 \beta}}\left(2 a-\operatorname{diam}\left(\mathbf{Z}_{n}\right)\right) \stackrel{\mathcal{D}}{\longrightarrow} \min _{i, j \geq 1}\left\{\mathcal{X}_{i, 1}+\mathcal{Y}_{j, 1}-\frac{1}{4 a}\left|\widetilde{\mathcal{X}}_{i}-\widetilde{\mathcal{Y}}_{j}\right|^{2}\right\}
$$

where $\left\{\mathcal{X}_{i}, i \geq 1\right\} \stackrel{\mathcal{D}}{=} \operatorname{PRM}\left(\alpha_{\ell} \cdot \Lambda_{\beta_{\ell}}^{*}\right)$ and $\left\{\mathcal{Y}_{j}, j \geq 1\right\} \stackrel{\mathcal{D}}{=} \operatorname{PRM}\left(\alpha_{r} \cdot \Lambda_{\beta_{r}}^{*}\right)$ are independent Poisson processes. If Condition 6 and - without loss of generality - the inequality $\beta_{\ell}>\beta_{r}$ hold true, we obtain

$$
n^{\frac{2}{d+1+2 \beta_{\ell}}}\left(2 a-\operatorname{diam}\left(\mathbf{Z}_{n}\right)\right) \stackrel{\mathcal{D}}{\longrightarrow} \min _{i \geq 1}\left\{\mathcal{X}_{i, 1}-\frac{1}{4 a}\left|\widetilde{\mathcal{X}}_{i}\right|^{2}\right\},
$$

with $\left\{\mathcal{X}_{i}, i \geq 1\right\} \stackrel{\mathcal{D}}{=} \operatorname{PRM}\left(\alpha_{\ell} \cdot \Lambda_{\beta_{\ell}}^{*}\right)$. The same results hold true if we replace $\operatorname{diam}\left(\mathbf{Z}_{n}\right)$ with $M_{n}$. 
The easiest class of distributions covered by Theorem 5.9 is obtained by choosing a set $E$ with a diameter of length $2 a>0$, fulfilling Conditions 1 to 3 , and densities of the form

$$
f_{\beta}^{*}(z):=\alpha \cdot\left(a-\left|z_{1}\right|\right)^{\beta} \cdot \mathbb{1}\left\{z \in E^{*}\right\},
$$

where $\beta>-\frac{d+1}{2}$ and $\alpha>0$, so that $\int_{E} f_{\beta}^{*}(z) \mathrm{d} z=1$, see the following example.

Example 5.10. Let $d=2, r>0$ and

$$
E:=B_{r}((-r, \mathbf{0})) \cup B_{r}((r, \mathbf{0}))
$$

This set is simply the union of two closed and touching two-dimensional balls with radii $r>0$ and centers $(-r, \mathbf{0})$ and $(r, \mathbf{0})$. Hence, it obviously fulfills Conditions 1 to 3 with $a=2 r$. For $\beta>-3 / 2$, we consider the densities

$$
f_{\beta}^{*}(z):=\alpha \cdot\left(2 r-\left|z_{1}\right|\right)^{\beta} \cdot \mathbb{1}\left\{z \in E^{*}\right\}
$$

with

$$
\alpha:=\frac{\Gamma(\beta+3)}{r^{\beta+2} 2^{\beta+3} \sqrt{\pi} \Gamma\left(\beta+\frac{3}{2}\right)} .
$$

In Subsection 5.2.3 we will show that $\int_{E} f_{\beta}^{*}(z) \mathrm{d} z=1$ holds true. The constant curvature of a circle with radius $r>0$ is $1 / r$, see Remark 6.5 for some more details. So, $\kappa_{2}^{\ell}=\kappa_{2}^{r}=1 / r$, and using the representation given in (3.16) yields

$$
P^{*}:=P^{*}\left(H_{\ell}\right)=P^{*}\left(H_{r}\right)=\left\{z \in \mathbb{R}^{2}: \frac{1}{2 r} \cdot z_{2}^{2} \leq z_{1}\right\} \backslash\{\mathbf{0}\} .
$$

Putting

$$
\Lambda_{\beta}^{*}(I):=\int_{I} z_{1}^{\beta} \mathbb{1}\left\{z \in P^{*}\right\} \mathrm{d} z
$$

for $I \in \mathcal{B}^{2}$, we can apply Theorem 5.9 with $a=2 r$ and obtain

$$
n^{\frac{2}{3+2 \beta}}\left(4 r-\operatorname{diam}\left(\mathbf{Z}_{n}\right)\right) \stackrel{\mathcal{D}}{\longrightarrow} \min _{i, j \geq 1}\left\{\mathcal{X}_{i, 1}+\mathcal{Y}_{j, 1}-\frac{1}{8 r}\left(\mathcal{X}_{i, 2}-\mathcal{Y}_{j, 2}\right)^{2}\right\},
$$

with independent Poisson processes $\left\{\mathcal{X}_{i}, i \geq 1\right\} \stackrel{\mathcal{D}}{=} \operatorname{PRM}\left(\alpha \cdot \Lambda_{\beta}^{*}\right)$ and $\left\{\mathcal{Y}_{j}, j \geq 1\right\} \stackrel{\mathcal{D}}{=}$ $\operatorname{PRM}\left(\alpha \cdot \Lambda_{\beta}^{*}\right)$.

The Figures $5.14,5.16,5.18$ and 5.20 illustrate the densities $f_{\beta}^{*}$ and those of the corresponding intensity measures $\alpha \cdot \Lambda_{\beta}^{*}$ for $r=1$ and $\beta \in\{-3 / 4,0,1,2\}$. For the same values of $r$ and $\beta$ we have performed a simulation study with 1000, 1000, 10000 and 100000 random points, respectively. The limit distributions have been approximated 
in the same way as described after Example 5.4. In this case, we obtained the approximating values $69.14,10.36,4.99$ and 3.7 , respectively, for the bound $b$ of the limiting processes. See Figures 55.15, 5.17, 5.19 and 5.21 for the results of this simulation study.
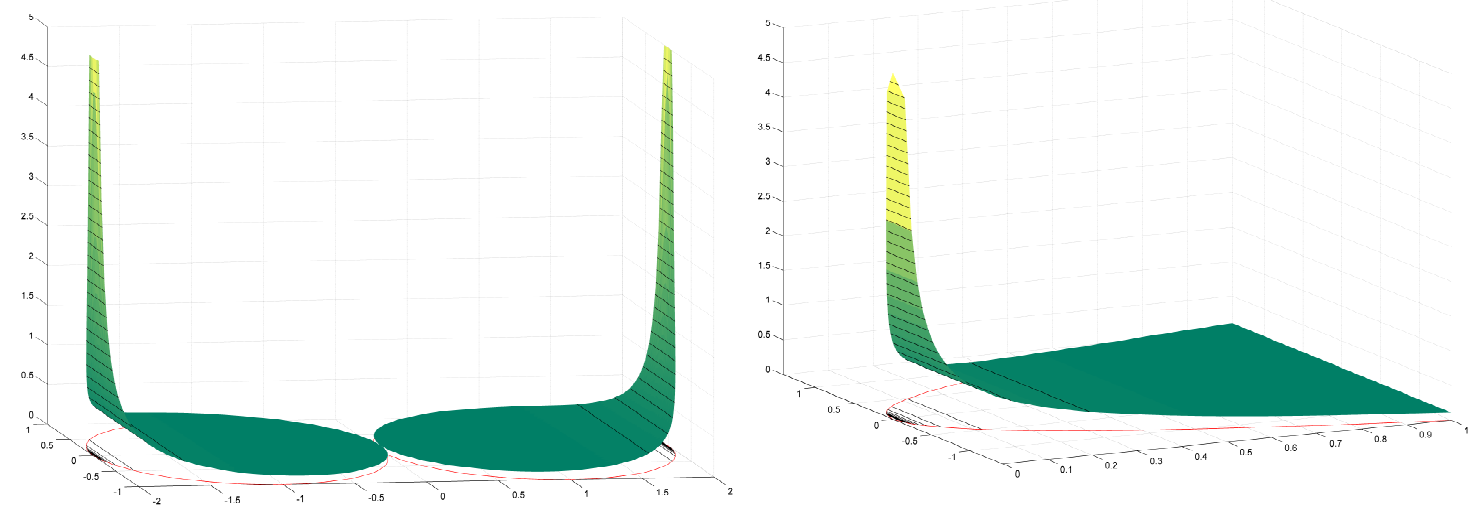

Figure 5.14: The density $f_{\beta}^{*}$ (left) and that of the intensity measure $\alpha \cdot \Lambda_{\beta}^{*}$ (right) in the setting of Example 5.10 with $r=1$ and $\beta=-3 / 4$.

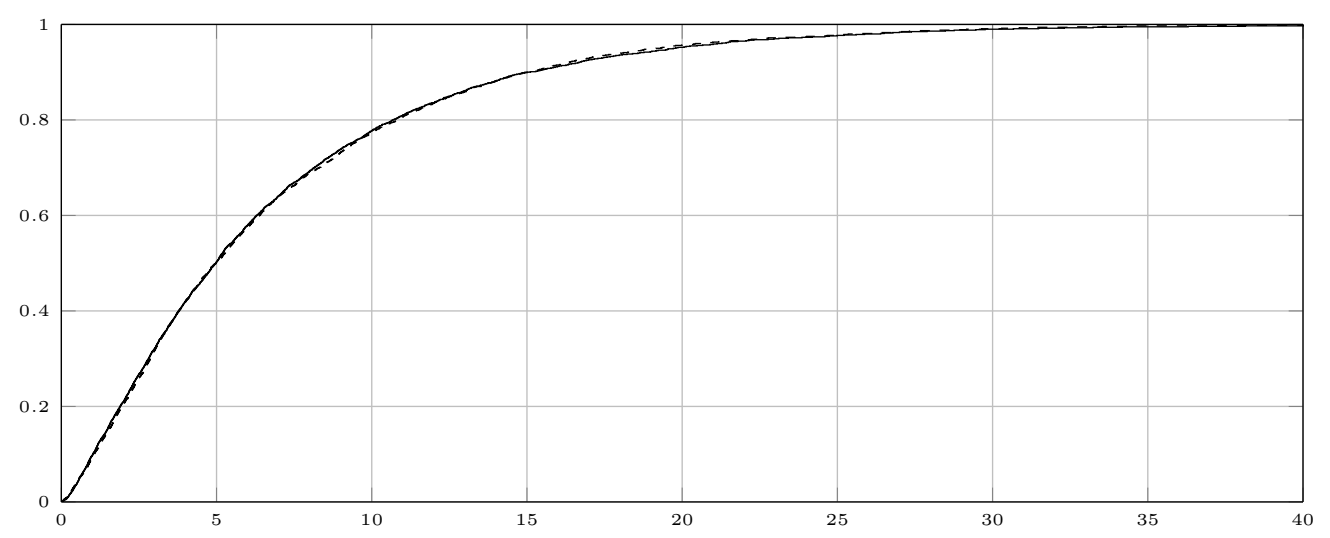

Figure 5.15: Empirical distribution function of $n^{4 / 3}\left(4-M_{n}\right)$ in the setting of Example 5.10 for $r=1, \beta=-3 / 4$ and $n=1000$ (solid, 5000 replications). The limit distribution is approximated in the same way as described after Example 5.4 with $b \approx 69.14$ (dashed, 5000 replications). 

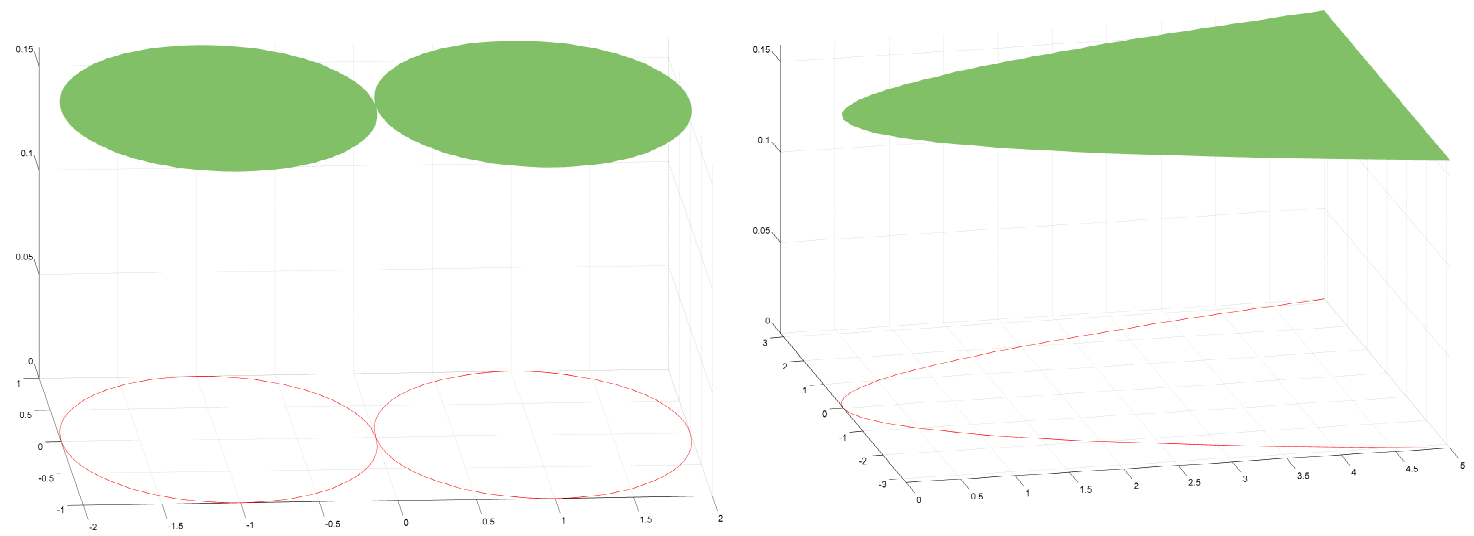

Figure 5.16: The density $f_{\beta}^{*}$ (left) and that of the intensity measure $\alpha \cdot \Lambda_{\beta}^{*}$ (right) in the setting of Example 5.10 with $r=1$ and $\beta=0$.

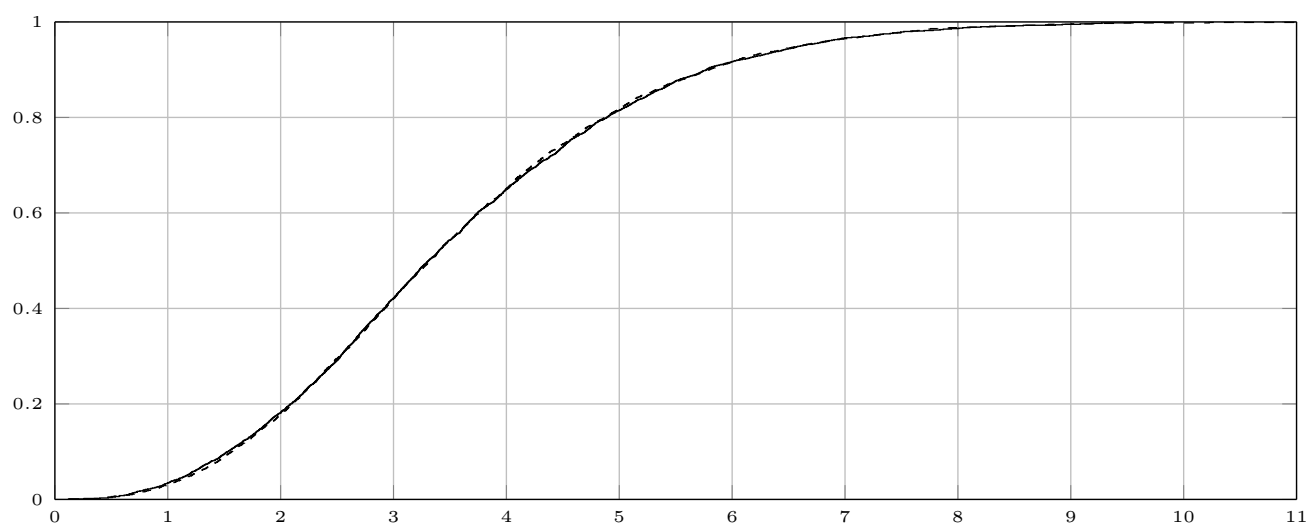

Figure 5.17: Empirical distribution function of $n^{2 / 3}\left(4-M_{n}\right)$ in the setting of Example 5.10 for $r=1, \beta=0$ and $n=1000$ (solid, 5000 replications). The limit distribution is approximated in the same way as described after Example 5.4 with $b \approx 10.36$ (dashed, 5000 replications).
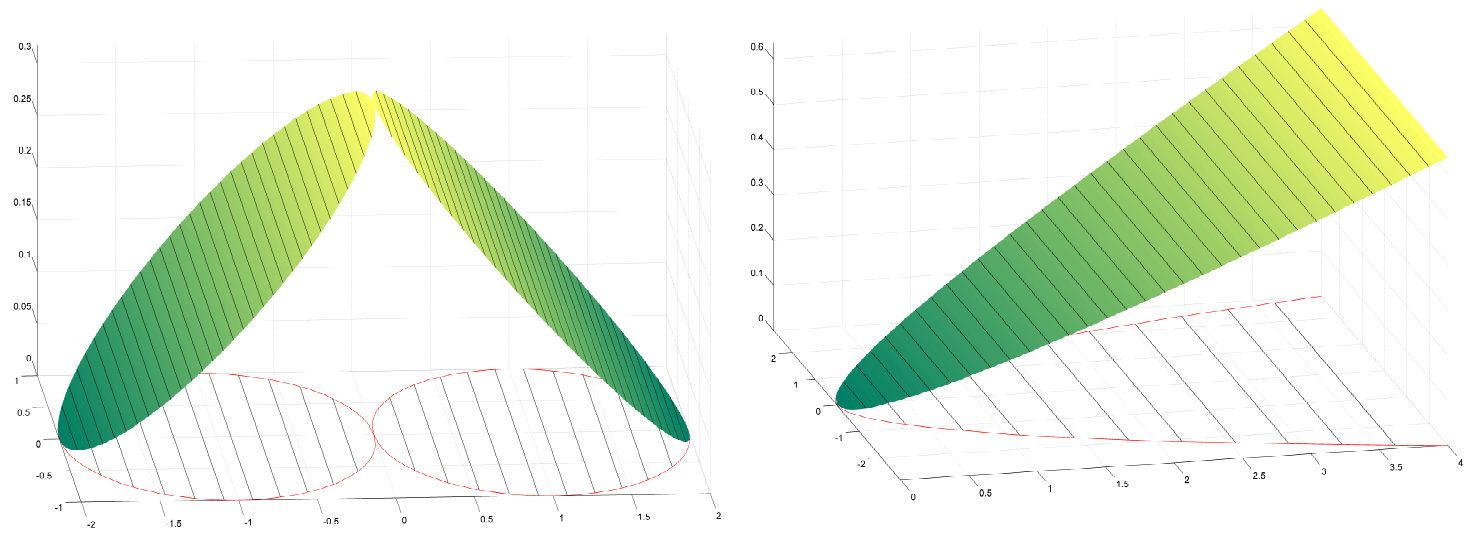

Figure 5.18: The density $f_{\beta}^{*}$ (left) and that of the intensity measure $\alpha \cdot \Lambda_{\beta}^{*}$ (right) in the setting of Example 5.10 with $r=1$ and $\beta=1$. 


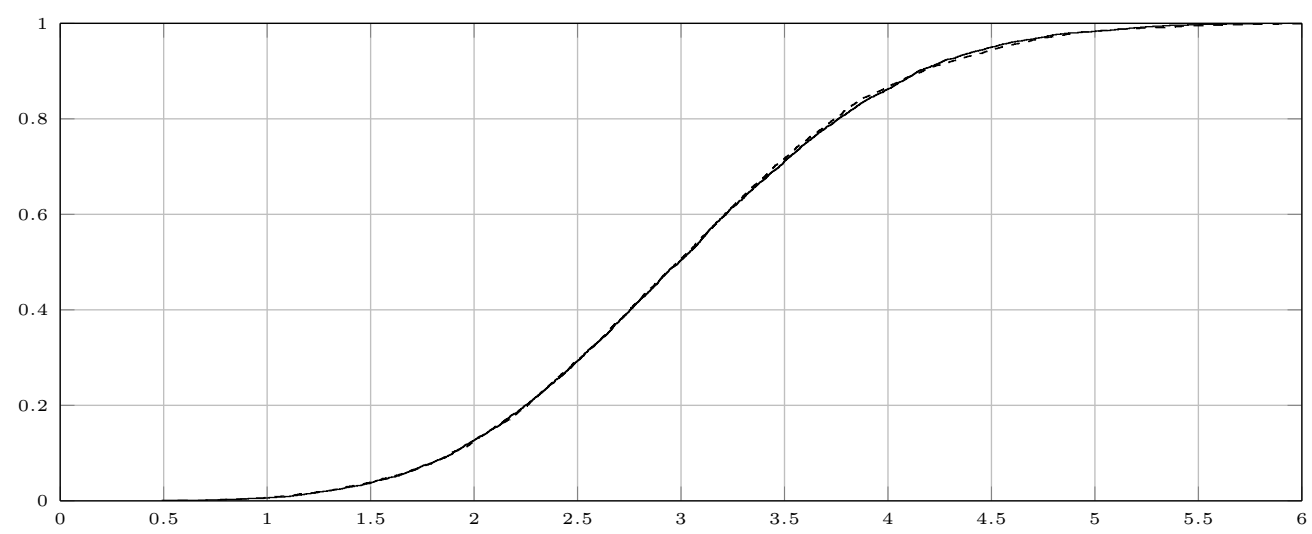

Figure 5.19: Empirical distribution function of $n^{2 / 5}\left(4-M_{n}\right)$ in the setting of Example 5.10 for $r=1, \beta=1$ and $n=10000$ (solid, 5000 replications). The limit distribution is approximated in the same way as described after Example 5.4 with $b \approx 4.99$ (dashed, 5000 replications).
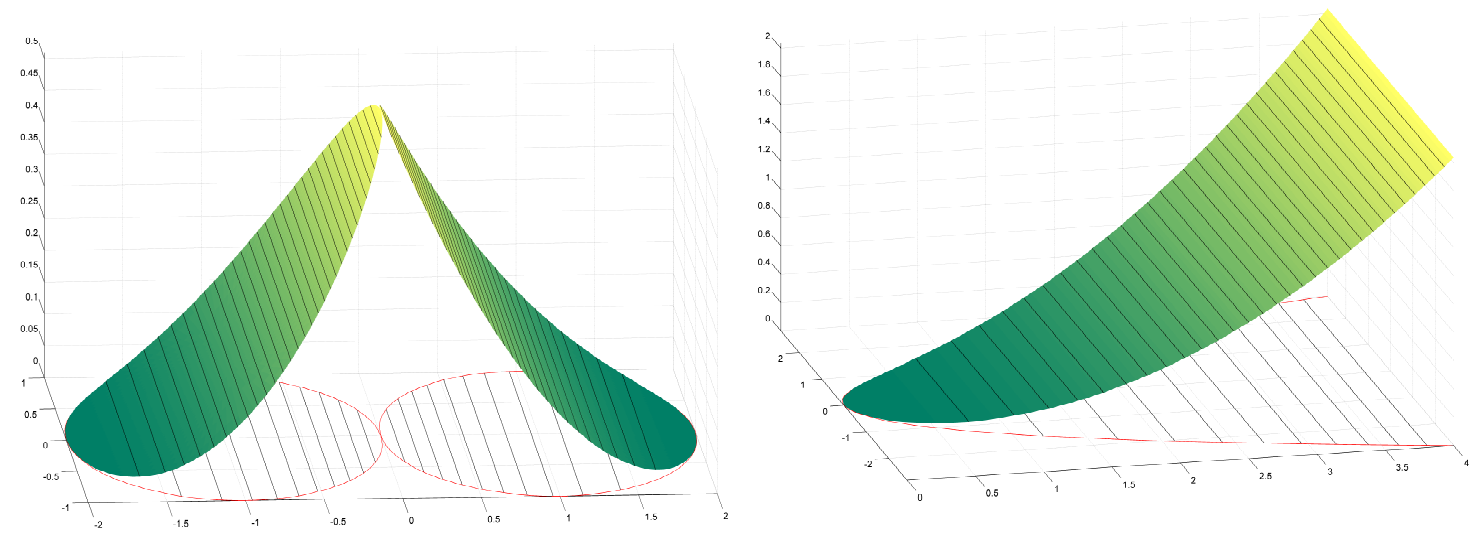

Figure 5.20: The density $f_{\beta}^{*}$ (left) and that of the intensity measure $\alpha \cdot \Lambda_{\beta}^{*}$ (right) in the setting of Example 5.10 with $r=1$ and $\beta=2$.

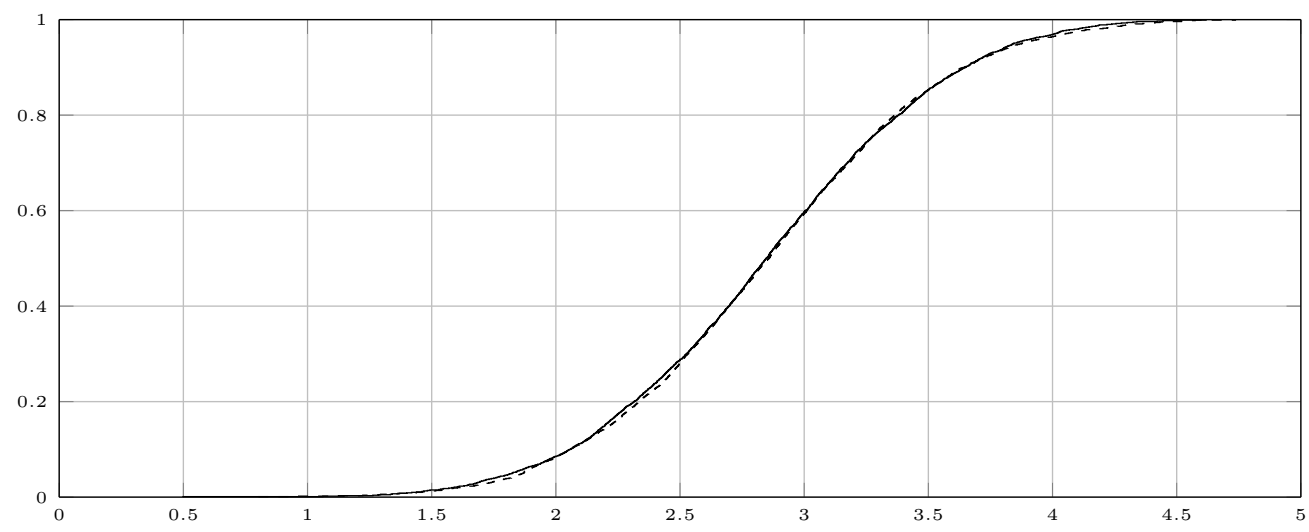

Figure 5.21: Empirical distribution function of $n^{2 / 7}\left(4-M_{n}\right)$ in the setting of Example 5.10 for $r=1, \beta=2$ and $n=100000$ (solid, 5000 replications). The limit distribution is approximated in the same way as described after Example 5.4 with $b \approx 3.7$ (dashed, 5000 replications). 


\subsubsection{Technical Details For SubSection 5.2.2}

In a first step we prove that the choice $\beta_{\ell}, \beta_{r}>-\frac{d+1}{2}$ in Condition 6 ensures the integrability of the function $f$ close to the poles. In a second step we will show that the constant $\alpha$ stated in Example 5.10 is correct.

Proof of the integrability of $f$ under Condition 6 close to the poles. It suffices to investigate the left pole-cap $E_{\ell, \delta}=E \cap\left\{z_{1} \leq-a+\delta\right\}, \delta \in\left(0, \delta_{\ell}\right)$. Since Conditions 1 to 3 hold true for $E$, we can find $a_{2}, \ldots, a_{d}>0$, so that

$$
E_{\ell, \delta} \subset\left\{z \in \mathbb{R}^{d}:\left(\frac{z_{1}}{a}\right)^{2}+\sum_{k=2}^{d}\left(\frac{z_{k}}{a_{k}}\right)^{2} \leq 1\right\}
$$

for sufficiently small $\delta>0$. Fixing $z_{1} \in(-a,-a+\delta)$ and putting $S\left(z_{1}\right):=$ $\left\{\widetilde{z} \in \mathbb{R}^{d-1}:\left(z_{1}, \widetilde{z}\right) \in E_{\ell, \delta}\right\}$, we especially get

$$
\begin{aligned}
S\left(z_{1}\right) & \subset\left\{\widetilde{z} \in \mathbb{R}^{d-1}: \sum_{k=2}^{d}\left(\frac{z_{k}}{a_{k}}\right)^{2} \leq 1-\left(\frac{z_{1}}{a}\right)^{2}\right\} \\
& =\left\{\widetilde{z} \in \mathbb{R}^{d-1}: \sum_{k=2}^{d}\left(\frac{z_{k}}{\frac{a_{k}}{a} \sqrt{a^{2}-z_{1}^{2}}}\right)^{2} \leq 1\right\} .
\end{aligned}
$$

Since the latter set is a $(d-1)$-dimensional ellipsoid with half-axes $\frac{a_{k}}{a} \sqrt{a^{2}-z_{1}^{2}}$, $k \in\{2, \ldots, d\}$, we obtain

$$
m_{d-1}\left(S\left(z_{1}\right)\right) \leq \omega_{d-1} \prod_{k=2}^{d} \frac{a_{k}}{a} \sqrt{a^{2}-z_{1}^{2}}=\omega_{d-1}\left(\prod_{k=2}^{d} \frac{a_{k}}{a}\right) \cdot\left(a^{2}-z_{1}^{2}\right)^{\frac{d-1}{2}}
$$

An application of Cavalieri's principle gives

$$
\begin{aligned}
\int_{E_{\ell, \delta}} \alpha_{\ell}\left(1+z_{1}\right)^{\beta_{\ell}} \mathrm{d} z & =\alpha_{\ell} \int_{-a}^{-a+\delta}\left(a+z_{1}\right)^{\beta_{\ell}} \int_{S\left(z_{1}\right)} 1 \mathrm{~d} y \mathrm{~d} z_{1} \\
& \leq \alpha_{\ell} \cdot \omega_{d-1}\left(\prod_{k=2}^{d} \frac{a_{k}}{a}\right) \cdot \int_{-a}^{-a+\delta}\left(a+z_{1}\right)^{\beta_{\ell}}\left(a^{2}-z_{1}^{2}\right)^{\frac{d-1}{2}} \mathrm{~d} z_{1} .
\end{aligned}
$$

Substituting $a+z_{1}=t$ yields $z_{1}^{2}=(t-a)^{2}=t^{2}-2 a t+a^{2}$, and hence

$$
\int_{E_{\ell, \delta}} \alpha_{\ell}\left(1+z_{1}\right)^{\beta_{\ell}} \mathrm{d} z \leq \alpha_{\ell} \cdot \omega_{d-1}\left(\prod_{k=2}^{d} \frac{a_{k}}{a}\right) \cdot \int_{0}^{\delta} t^{\beta_{\ell}}\left(2 a t-t^{2}\right)^{\frac{d-1}{2}} \mathrm{~d} z_{1} .
$$


Since $t^{\beta_{\ell}}\left(2 a t-t^{2}\right)^{\frac{d-1}{2}}=(2 a)^{\frac{d-1}{2}} t^{\beta_{\ell}+\frac{d-1}{2}}+O\left(t^{\beta_{\ell}+\frac{d-1}{2}+1}\right)$ as $t \downarrow 0$, this upper bound is finite, whenever we have $\beta_{\ell}+\frac{d-1}{2}>-1$, i.e. $\beta_{\ell}>-\frac{d+1}{2}$. So, $f$ is integrable close to the poles.

Verification of the constant stated in Example 5.10. Using the symmetries of $E=$ $B_{r}((-r, \mathbf{0})) \cup B_{r}((r, \mathbf{0})) \subset \mathbb{R}^{2}$, we have

$$
\begin{aligned}
\int_{E} f_{\beta}^{*}(z) \mathrm{d} z & =\alpha \cdot 2 \cdot \int_{0}^{2 r} 2 \int_{0}^{\sqrt{r^{2}-\left(z_{1}-r\right)^{2}}}\left(2 r-z_{1}\right)^{\beta} \mathrm{d} z_{2} \mathrm{~d} z_{1} \\
& =\alpha \cdot 4 \int_{0}^{2 r} \sqrt{r^{2}-\left(z_{1}-r\right)^{2}}\left(2 r-z_{1}\right)^{\beta} \mathrm{d} z_{1} \\
& =\alpha \cdot 4 \int_{0}^{2 r} \sqrt{2 z_{1} r-z_{1}^{2}}\left(2 r-z_{1}\right)^{\beta} \mathrm{d} z_{1} \\
& =\alpha \cdot 4 \int_{0}^{2 r} \sqrt{z_{1}}\left(2 r-z_{1}\right)^{\beta+\frac{1}{2}} \mathrm{~d} z_{1} \\
& =\alpha \cdot 4 \cdot(2 r)^{\beta+\frac{1}{2}} \int_{0}^{2 r} \sqrt{z_{1}}\left(1-\frac{z_{1}}{2 r}\right)^{\beta+\frac{1}{2}} \mathrm{~d} z_{1} .
\end{aligned}
$$

Substituting $z_{1} / 2 r=t$ yields $\mathrm{d} z_{1}=2 r \mathrm{~d} t$ and hence

$$
\begin{aligned}
\int_{E} f_{\beta}^{*}(z) \mathrm{d} z & =\alpha \cdot 4 \cdot(2 r)^{\beta+\frac{1}{2}} \int_{0}^{1} \sqrt{2 r t}(1-t)^{\beta+\frac{1}{2}} 2 r \mathrm{~d} t \\
& =\alpha \cdot 4 \cdot(2 r)^{\beta+2} \int_{0}^{1} \sqrt{t}(1-t)^{\beta+\frac{1}{2}} \mathrm{~d} t \\
& =\alpha \cdot 4 \cdot(2 r)^{\beta+2} B\left(\frac{3}{2}, \beta+\frac{3}{2}\right) \\
& =\alpha \cdot 4 \cdot(2 r)^{\beta+2} \frac{\Gamma\left(\frac{3}{2}\right) \Gamma\left(\beta+\frac{3}{2}\right)}{\Gamma(\beta+3)} \\
& =\alpha \cdot \frac{4 \cdot(2 r)^{\beta+2} \cdot \frac{1}{2} \cdot \sqrt{\pi} \Gamma\left(\beta+\frac{3}{2}\right)}{\Gamma(\beta+3)} \\
& =\alpha \cdot \frac{r^{\beta+2} 2^{\beta+3} \sqrt{\pi} \Gamma\left(\beta+\frac{3}{2}\right)}{\Gamma(\beta+3)} \\
& =1 .
\end{aligned}
$$




\subsection{JOINT CONVERGENCE OF THE $k$ LARGEST DistANCES}

To state a result on the joint asymptotical behavior of the $k$ largest distances of the Poisson process $\mathbf{Z}_{n}=\sum_{i=1}^{N_{n}} \varepsilon_{Z_{i}}$, introduced in Section 2.2, we need some additional definitions. For $n \in \mathbb{N}$, let $D_{n}^{(1)} \geq D_{n}^{(2)} \geq \ldots \geq D_{n}^{(k)}$ be the $k$ largest distances in descending order between $Z_{i}$ and $Z_{j}$ for $1 \leq i<j \leq N_{n}$. So, we especially have $D_{n}^{(1)}=\operatorname{diam}\left(\mathbf{Z}_{n}\right)$. For a point process $\xi$ on $\mathbb{R}_{+}$and $i \in \mathbb{N}$ we define $t_{i}(\xi):=\inf \{t: \xi([0, t]) \geq i\}$. According to Proposition 9.1.XII in Daley and VereJones [6], each $t_{i}(\xi)$ is a well-defined random variable if $\xi$ is a simple point process. Since the point processes $\widehat{G}\left(\mathbf{X}_{n} \times \mathbf{Y}_{n}\right)$ and $\widehat{G}(\mathbf{X} \times \mathbf{Y})$ on $\mathbb{R}_{+}$(introduced in the proof of Theorem 3.5) are simple, we conclude that the random variables $t_{i}\left(\widehat{G}\left(\mathbf{X}_{n} \times \mathbf{Y}_{n}\right)\right)$ and $t_{i}(\widehat{G}(\mathbf{X} \times \mathbf{Y}))$ are well-defined for each fixed $i \in \mathbb{N}$. Now we can state our result on the joint convergence of the $k$ largest distances in the setting of Chapter 3 :

Theorem 5.11. If Conditions 1 to 4 hold true, we have for each $k \in \mathbb{N}$ the joint convergence

$$
n^{\frac{2}{d+1}}\left(2 a-D_{n}^{(1)}, \ldots, 2 a-D_{n}^{(k)}\right) \stackrel{\mathcal{D}}{\longrightarrow}\left(t_{1}(\widehat{G}(\mathbf{X} \times \mathbf{Y})), \ldots, t_{k}(\widehat{G}(\mathbf{X} \times \mathbf{Y}))\right),
$$

where $\mathbf{X} \stackrel{\mathcal{D}}{=} P R M\left(\left.p_{\ell} \cdot m_{d}\right|_{P\left(H_{\ell}\right)}\right)$ and $\mathbf{Y} \stackrel{\mathcal{D}}{=} P R M\left(\left.p_{r} \cdot m_{d}\right|_{P\left(H_{r}\right)}\right)$ are independent Poisson processes.

Proof. Using the same arguments as in the proof of Theorem 3.5, we only have to show that

$$
\begin{aligned}
& \left(t_{1}\left(\widehat{G}\left(\mathbf{X}_{n} \times \mathbf{Y}_{n}\right)\right), \ldots, t_{k}\left(\widehat{G}\left(\mathbf{X}_{n} \times \mathbf{Y}_{n}\right)\right)\right) \\
\stackrel{\mathcal{D}}{\longrightarrow} & \left(t_{1}(\widehat{G}(\mathbf{X} \times \mathbf{Y})), \ldots, t_{k}(\widehat{G}(\mathbf{X} \times \mathbf{Y}))\right) .
\end{aligned}
$$

We briefly write $\xi_{n}:=\widehat{G}\left(\mathbf{X}_{n} \times \mathbf{Y}_{n}\right)$ and $\xi:=\widehat{G}(\mathbf{X} \times \mathbf{Y})$. Then, (4.17) means $\xi_{n} \stackrel{\mathcal{D}}{\longrightarrow} \xi$, and from Theorem 16.16 in Kallenberg [15] (recapitulated as Theorem B.1 in Section B.3) we get

$$
\left(\xi_{n}\left(B_{1}\right), \ldots, \xi_{n}\left(B_{k}\right)\right) \stackrel{\mathcal{D}}{\longrightarrow}\left(\xi\left(B_{1}\right), \ldots, \xi\left(B_{k}\right)\right)
$$

for any choice of bounded intervals $B_{1}, \ldots, B_{k} \subset \mathbb{R}_{+}$with $\xi\left(\partial B_{i}\right)=0$ almost surely, $i \in\{1, \ldots, k\}$. Let $0<s_{1}<\ldots<s_{k}$ be arbitrary. By use of the inclusion-exclusion 
principle we obtain

$$
\begin{aligned}
& \mathbb{P}\left(t_{1}\left(\xi_{n}\right) \leq s_{1}, \ldots, t_{k}\left(\xi_{n}\right) \leq s_{k}\right) \\
= & 1-\mathbb{P}\left(\left\{t_{1}\left(\xi_{n}\right) \leq s_{1}, \ldots, t_{k}\left(\xi_{n}\right) \leq s_{k}\right\}^{c}\right) \\
= & 1-\sum_{r=1}^{k}(-1)^{r-1} \sum_{1 \leq i_{1}<\ldots<i_{r} \leq k} \mathbb{P}\left(\left\{t_{i_{1}}\left(\xi_{n}\right) \leq s_{i_{1}}\right\}^{c} \cap \ldots \cap\left\{t_{i_{r}}\left(\xi_{n}\right) \leq s_{i_{r}}\right\}^{c}\right) \\
\rightarrow & 1-\sum_{r=1}^{k}(-1)^{r-1} \sum_{1 \leq i_{1}<\ldots<i_{r} \leq k} \mathbb{P}\left(\left\{t_{i_{1}}(\xi) \leq s_{i_{1}}\right\}^{c} \cap \ldots \cap\left\{t_{i_{r}}(\xi) \leq s_{i_{r}}\right\}^{c}\right) \\
= & 1-\mathbb{P}\left(\left\{t_{1}(\xi) \leq s_{1}, \ldots, t_{k}(\xi) \leq s_{k}\right\}^{c}\right) \\
= & \mathbb{P}\left(t_{1}(\xi) \leq s_{1}, \ldots, t_{k}(\xi) \leq s_{k}\right),
\end{aligned}
$$

and (5.26) is shown. Notice thereby: Since $\xi_{n}$ are point processes, we know that for every choice of $i_{j} \in \mathbb{N}$ the point $i_{j}-\frac{1}{2}$ is a point of continuity of the distribution function of $\xi_{n}\left(\left[0, s_{i_{j}}\right]\right)$. Furthermore, we have

$$
\xi_{n}\left(\left[0, s_{i_{j}}\right]\right)<i_{j} \quad \Longleftrightarrow \quad \xi_{n}\left(\left[0, s_{i_{j}}\right]\right) \leq i_{j}-\frac{1}{2} .
$$

The same holds true if we replace $\xi_{n}$ with $\xi$. As a consequence of (5.27) we get for all $1 \leq i_{1}<\ldots<i_{r} \leq k$

$$
\begin{aligned}
& \mathbb{P}\left(\left\{t_{i_{1}}\left(\xi_{n}\right) \leq s_{i_{1}}\right\}^{c} \cap \ldots \cap\left\{t_{i_{r}}\left(\xi_{n}\right) \leq s_{i_{r}}\right\}^{c}\right) \\
= & \mathbb{P}\left(t_{i_{1}}\left(\xi_{n}\right)>s_{i_{1}}, \ldots, t_{i_{r}}\left(\xi_{n}\right)>s_{i_{r}}\right) \\
= & \mathbb{P}\left(\xi_{n}\left(\left[0, s_{i_{1}}\right]\right)<i_{1}, \xi_{n}\left(\left[0, s_{i_{2}}\right]\right)<i_{2}, \ldots, \xi_{n}\left(\left[0, s_{i_{r}}\right]\right)<i_{r}\right) \\
= & \mathbb{P}\left(\xi_{n}\left(\left[0, s_{i_{1}}\right]\right) \leq i_{1}-\frac{1}{2}, \xi_{n}\left(\left[0, s_{i_{2}}\right]\right) \leq i_{2}-\frac{1}{2}, \ldots, \xi_{n}\left(\left[0, s_{i_{r}}\right]\right) \leq i_{r}-\frac{1}{2}\right) \\
\rightarrow & \mathbb{P}\left(\xi\left(\left[0, s_{i_{1}}\right]\right) \leq i_{1}-\frac{1}{2}, \xi\left(\left[0, s_{i_{2}}\right]\right) \leq i_{2}-\frac{1}{2}, \ldots, \xi\left(\left[0, s_{i_{r}}\right]\right) \leq i_{r}-\frac{1}{2}\right) \\
= & \mathbb{P}\left(\xi\left(\left[0, s_{i_{1}}\right]\right)<i_{1}, \xi\left(\left[0, s_{i_{2}}\right]\right)<i_{2}, \ldots, \xi\left(\left[0, s_{i_{r}}\right]\right)<i_{r}\right) \\
= & \mathbb{P}\left(t_{i_{1}}(\xi)>s_{i_{1}}, \ldots, t_{i_{r}}(\xi)>s_{i_{r}}\right) \\
= & \mathbb{P}\left(\left\{t_{i_{1}}(\xi) \leq s_{i_{1}}\right\}^{c} \cap \ldots \cap\left\{t_{i_{r}}(\xi) \leq s_{i_{r}}\right\}^{c}\right) .
\end{aligned}
$$

Using exactly the same arguments, we can immediately generalize the results of Section 5.1 and Section 5.2, too. Since the necessary adjustments are obvious, we will only state a generalized result for distributions covered by the setting given in 


\section{Section 5.1.}

Theorem 5.12. Let the density $f$ be supported by the ellipsoid $E$ defined in (5.1) with half-axes $a_{1}>a_{2} \geq \ldots \geq a_{d}>0$, and put $a:=a_{1}$. If $f$ satisfies Condition 5 with $\beta_{\ell}=\beta_{r}=: \beta$ then, for each fixed $k \geq 1$, we have

$n^{\frac{2}{d+1+2 \beta}}\left(2 a-D_{n}^{(1)}, \ldots, 2 a-D_{n}^{(k)}\right) \stackrel{\mathcal{D}}{\longrightarrow}\left(t_{1}(\widehat{G}(\mathbf{X} \times \mathbf{Y})), \ldots, t_{k}(\widehat{G}(\mathbf{X} \times \mathbf{Y}))\right)$ where $\mathbf{X} \stackrel{\mathcal{D}}{=} \operatorname{PRM}\left(\alpha_{\ell} \cdot \Lambda_{\beta}\right)$ and $\mathbf{Y} \stackrel{\mathcal{D}}{=} \operatorname{PRM}\left(\alpha_{r} \cdot \Lambda_{\beta}\right)$ are independent Poisson processes.

Notice that the definition $a:=a_{1}$ in the theorem above is necessary, since the function $\widehat{G}$ has been defined in Section 4.3 in terms of $a$, not of $a_{1}$.

We now present the results of a simulation study in the setting of Theorem 5.12 for $d=2, a_{1}=1, a_{2}=1 / 2, \beta \in\{-1 / 2,0,1\}$ and $k=10$. Since there is no way to visualize the joint convergence of the ten largest distances, we have calculated for $i \in\{1, \ldots, 10\}$ the (univariate) empirical distribution function $\widehat{F}_{n}^{(i)}$ of $n^{2 /(3+2 \beta)}\left(2-D_{n}^{(i)}\right)$, and these ten functions have been plotted side by side. Because of $2 \geq D_{n}^{(1)} \geq \ldots \geq D_{n}^{(10)}$, we have $0 \leq 2-D_{n}^{(1)} \leq \ldots \leq 2-D_{n}^{(10)}$ and hence $\widehat{F}_{n}^{(1)} \geq \ldots \geq \widehat{F}_{n}^{(10)}$ for each $n \in \mathbb{N}$. As in the simulation study after Example 5.4, we have chosen $n=1000$ for $\beta \in\{-1 / 2,0\}$ and $n=10000$ for $\beta=1$. The (one-dimensional) limit distributions $t_{1}(\widehat{G}(\mathbf{X} \times \mathbf{Y})), \ldots, t_{10}(\widehat{G}(\mathbf{X} \times \mathbf{Y}))$ have been approximated by simulating $t_{1}\left(\widehat{G}\left(\mathbf{X}_{b} \times \mathbf{Y}_{b}\right)\right), \ldots, t_{10}\left(\widehat{G}\left(\mathbf{X}_{b} \times \mathbf{Y}_{b}\right)\right)$ where $\xi_{b}$ denotes the restriction of the point process $\xi$ to the set $\left\{z \in \mathbb{R}^{2}: z_{1} \leq b\right\}, b>0$. As in the simulation study after Example 5.4, $b$ had to be chosen subject to $\beta$. Like before, the values $20,6.52$ and 2.55 for $\beta=-1 / 2,0,1$, respectively, were sufficient for a good approximation in this context. The Figures 5.5, 5.7 and 5.9 have already illustrated a good match between $\widehat{F}_{n}^{(1)}$ and the empirical distribution function of $t_{1}\left(\widehat{G}\left(\mathbf{X}_{b} \times \mathbf{Y}_{b}\right)\right)$ in this setting. Likewise, Figures 5.22 to 5.24 reveal that the chosen numbers $n$ of random points and values for $b$ are sufficient to obtain a very good approximation for all ten components.

This componentwise point of view does not provide any insight into the probabilistic connection between the largest distances. In order to obtain an impression of the joint behavior of the largest and the second largest distance, we want to illustrate the (approximated) joint densities of $n^{\frac{2}{3+2 \beta}}\left(2-D_{n}^{(1)}, 2-D_{n}^{(2)}\right)$ in the setting of Theorem 5.12 for $d=2, a_{1}=1, a_{2}=1 / 2$ and $\beta \in\{-1 / 2,0,1\}$. As in the simulation study before, we have chosen $n=1000$ if $\beta \in\{-1 / 2,0\}$, and for $\beta=1$ the 


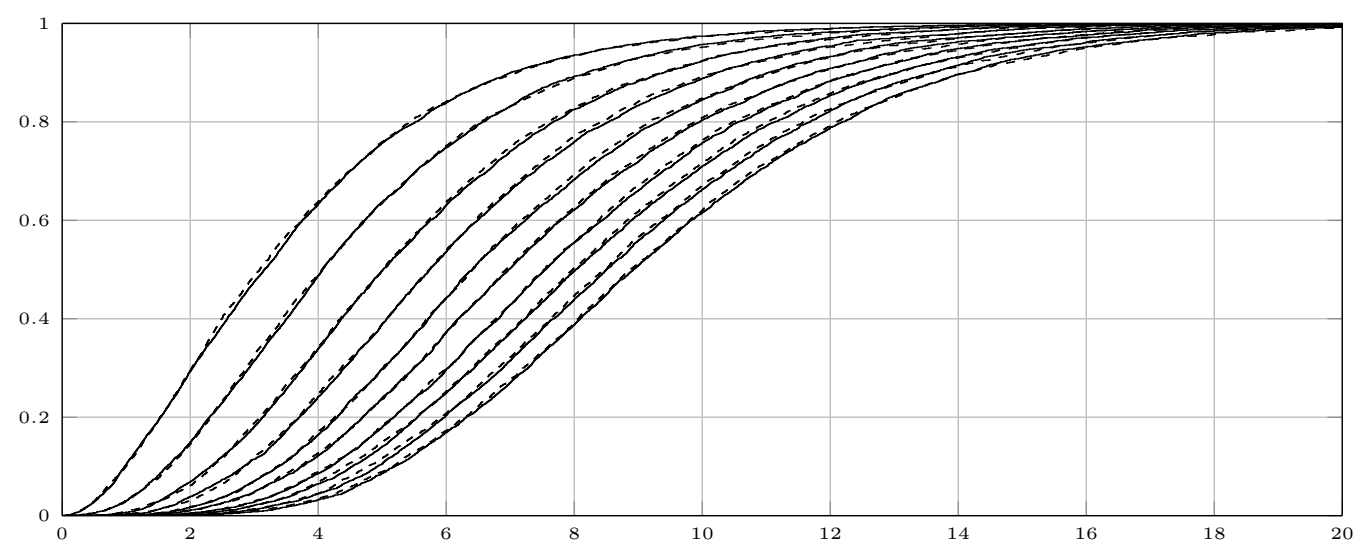

Figure 5.22: Empirical distribution functions of $n\left(2-D_{n}^{(1)}\right), \ldots, n\left(2-D_{n}^{(10)}\right)$ in the setting of Theorem 5.12 for $d=2$ with $a_{1}=1, a_{2}=1 / 2, \beta_{\ell}=\beta_{r}=$ $-1 / 2$ and $n=1000$ (solid, from left to right, 5000 replications). The corresponding limit distributions are approximated, as described after Theorem 5.12, with $b=20$ (dashed, 5000 replications).

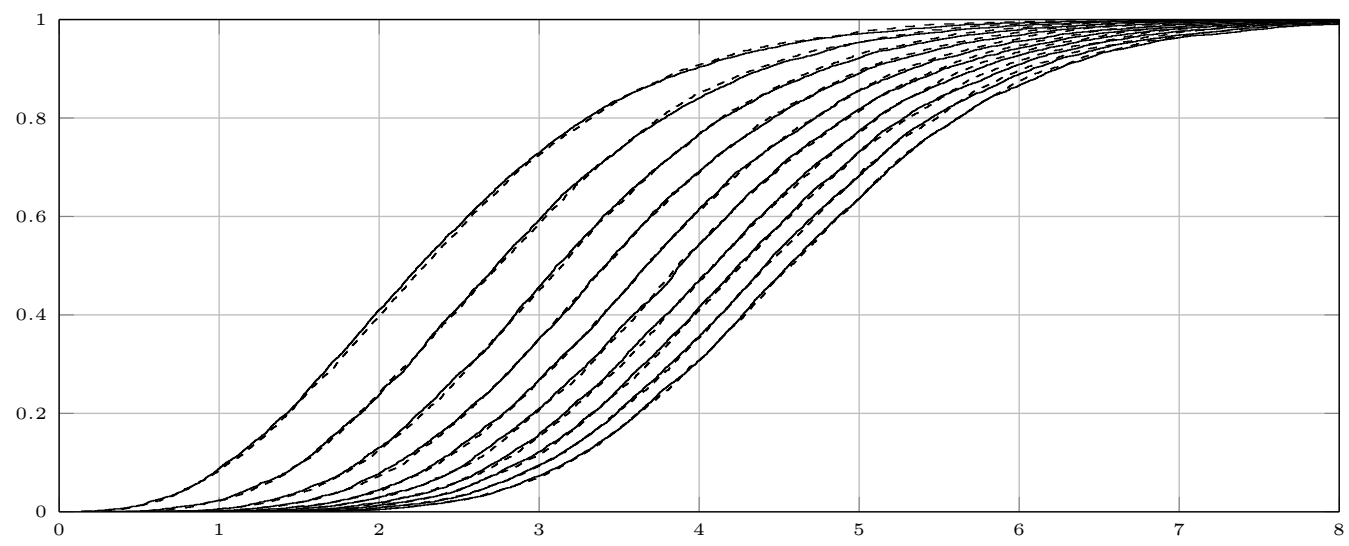

Figure 5.23: Empirical distribution functions of $n^{2 / 3}\left(2-D_{n}^{(1)}\right), \ldots, n^{2 / 3}\left(2-D_{n}^{(10)}\right)$ in the setting of Theorem 5.12 for $d=2$ with $a_{1}=1, a_{2}=1 / 2, \beta_{\ell}=$ $\beta_{r}=0$ and $n=1000$ (solid, from left to right, 5000 replications). The corresponding limit distributions are approximated, as described after Theorem 5.12, with $b \approx 6.52$ (dashed, 5000 replications).

underlying number of random points is $n=10000$. These numbers of random points have shown a good approximation of the limiting processes in the simulation study before. Since $0 \leq 2-D_{n}^{(1)} \leq 2-D_{n}^{(2)}$ for every $n \in \mathbb{N}$, the joint density of $n^{\frac{2}{3+2 \beta}}\left(2-D_{n}^{(1)}, 2-D_{n}^{(2)}\right)$ has support $\left\{z \in \mathbb{R}^{2}: 0 \leq z_{1} \leq z_{2}\right\}$. For $\beta \in\{-1 / 2,0,1\}$, this density has been approximated by independently simulating 1000000 realisations of $n^{\frac{2}{3+2 \beta}}\left(2-D_{n}^{(1)}, 2-D_{n}^{(2)}\right)$, and then applying a kernel density estimator. The results are illustrated in the Figures 5.25 to 5.27 . Observe the very different scalings between these three figures. 


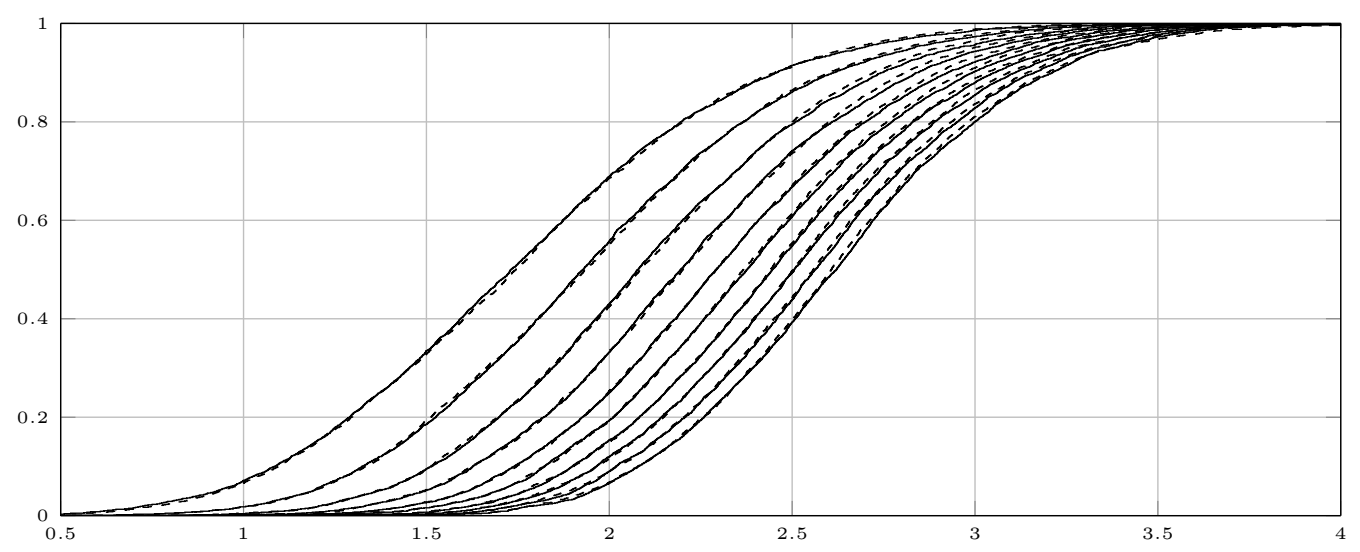

Figure 5.24: Empirical distribution functions of $n^{2 / 5}\left(2-D_{n}^{(1)}\right), \ldots, n^{2 / 5}\left(2-D_{n}^{(10)}\right)$ in the setting of Theorem 5.12 for $d=2$ with $a_{1}=1, a_{2}=1 / 2, \beta_{\ell}=\beta_{r}=1$ and $n=10000$ (solid, from left to right, 5000 replications). The corresponding limit distributions are approximated, as described after Theorem 5.12, with $b \approx 2.55$ (dashed, 5000 replications).

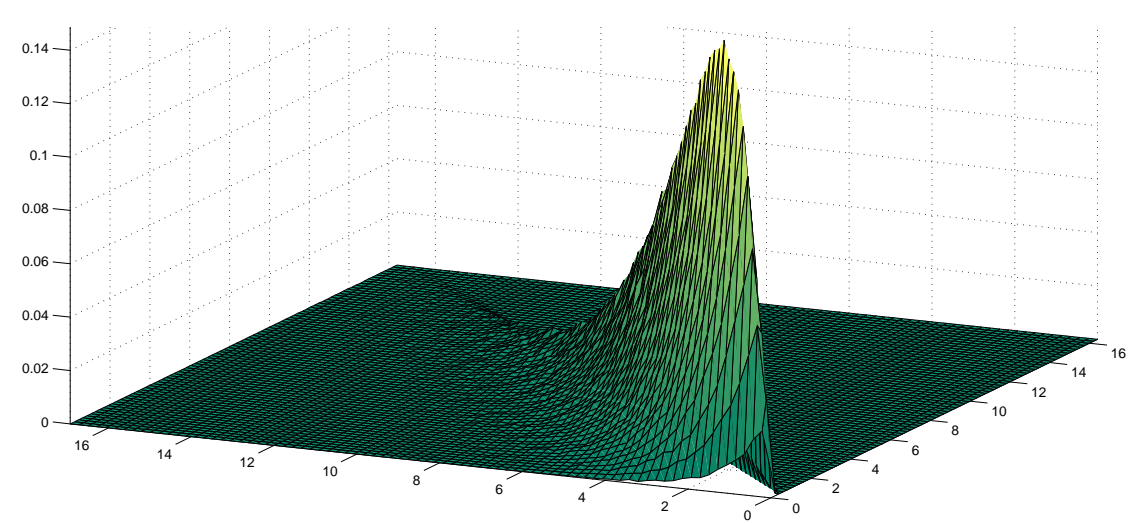

Figure 5.25: The (approximated) joint density of $n\left(2-D_{n}^{(1)}, 2-D_{n}^{(2)}\right)$ in the setting of Theorem 5.12 for $d=2$ with $a_{1}=1, a_{2}=1 / 2, \beta_{\ell}=\beta_{r}=-1 / 2$ and $n=1000$ (1000000 replications).

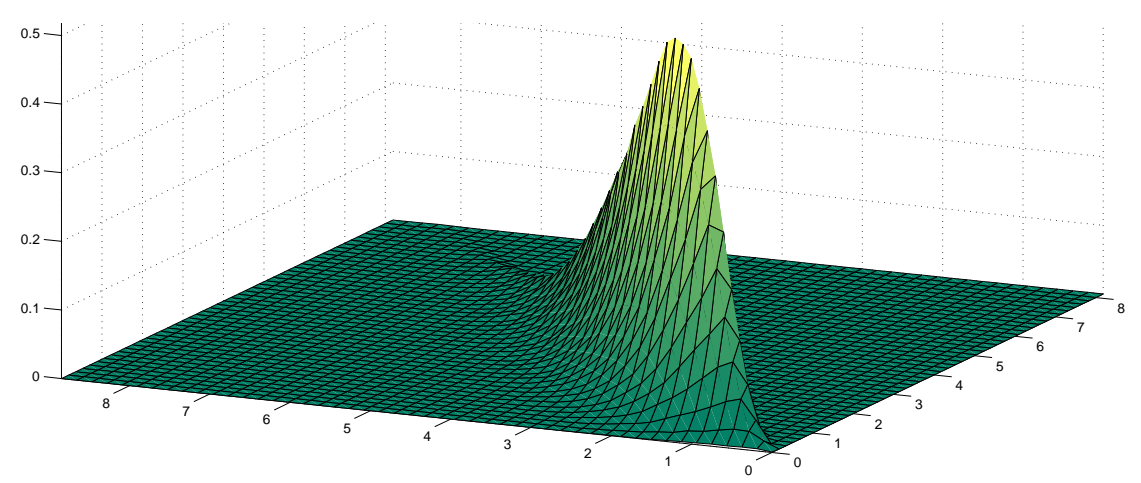

Figure 5.26: The (approximated) joint density of $n^{2 / 3}\left(2-D_{n}^{(1)}, 2-D_{n}^{(2)}\right)$ in the setting of Theorem 5.12 for $d=2$ with $a_{1}=1, a_{2}=1 / 2, \beta_{\ell}=\beta_{r}=0$ and $n=1000$ (1000000 replications). 


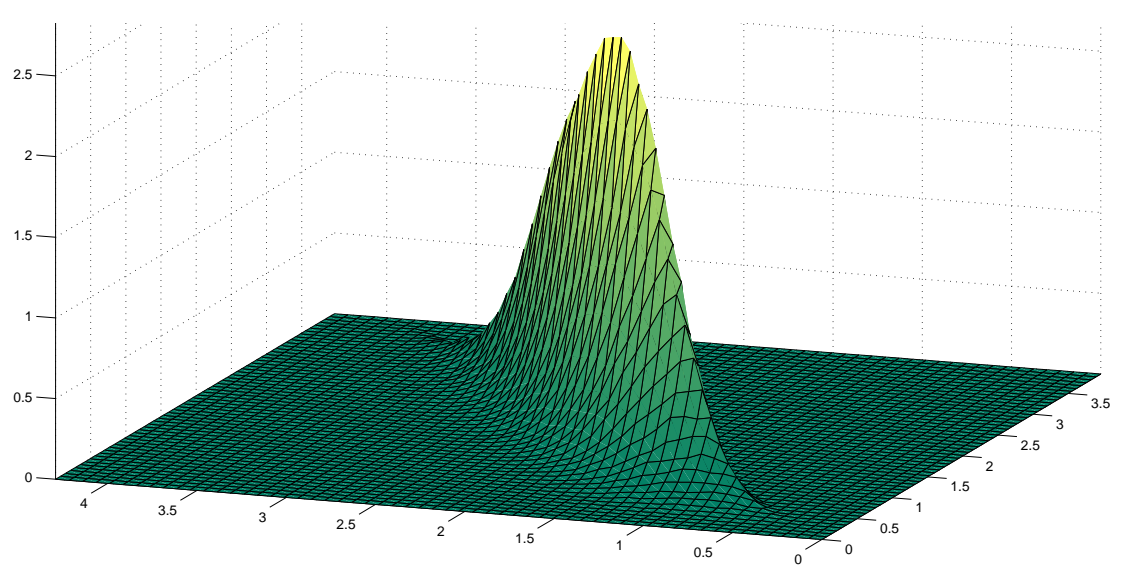

Figure 5.27: The (approximated) joint density of $n^{2 / 5}\left(2-D_{n}^{(1)}, 2-D_{n}^{(2)}\right)$ in the setting of Theorem 5.12 for $d=2$ with $a_{1}=1, a_{2}=1 / 2, \beta_{\ell}=\beta_{r}=1$ and $n=10000$ (1000000 replications).

\subsection{A Different Shape of $E$ Close to The POLES}

In this section, we replace Condition 2 with the following one:

Condition 7. There are constants $\delta_{\ell}, \delta_{r} \in(0, a]$, open neighborhoods $O_{\ell}, O_{r} \subset \mathbb{R}^{d-1}$ of $\mathbf{0} \in \mathbb{R}^{d-1}$ and twice continuously differentiable functions $s^{\ell, 1}, s^{\ell, 2}: O_{\ell} \rightarrow \mathbb{R}_{+}$, $s^{r, 1}, s^{r, 2}: O_{r} \rightarrow \mathbb{R}_{+}$with $s^{i, 1}(\mathbf{0})=s^{i, 2}(\mathbf{0})=0$ and $s^{i, 1}(\widetilde{z})<s^{i, 2}(\widetilde{z})$ for $\widetilde{z} \in O_{i} \backslash\{\mathbf{0}\}$, $i \in\{\ell, r\}$, so that

$$
\begin{aligned}
E_{\ell} & :=E \cap\left\{z_{1}<-a+\delta_{\ell}\right\} \\
& =\left\{\left(z_{1}, \widetilde{z}\right) \in \mathbb{R}^{d}:-a+s^{\ell, 1}(\widetilde{z}) \leq z_{1} \leq-a+s^{\ell, 2}(\widetilde{z}), z_{1}<-a+\delta_{\ell}, \widetilde{z} \in O_{\ell}\right\}
\end{aligned}
$$

and

$$
\begin{aligned}
E_{r} & :=E \cap\left\{a-\delta_{r}<z_{1}\right\} \\
& =\left\{\left(z_{1}, \widetilde{z}\right) \in \mathbb{R}^{d}: a-s^{r, 2}(\widetilde{z}) \leq z_{1} \leq a-s^{r, 1}(\widetilde{z}), a-\delta_{r}<z_{1}, \widetilde{z} \in O_{r}\right\} .
\end{aligned}
$$

Figure 5.28 illustrates the new shape of $E$ close to the poles.

In this setting we can apply the same reasoning as given after Condition 2 to the functions $s^{i, 2}$, describing the 'inner boundary of $E_{i}$ ' for $i \in\{\ell, r\}$. To this end, we have to introduce a more lengthy notation as in Chapter 3: For $i \in\{\ell, r\}$ and $j \in\{1,2\}$ we write $H_{i, j}$ for the Hessian of $s^{i, j}$ at the corresponding pole. Its eigenvalues are called $\kappa_{2}^{i, j}, \ldots, \kappa_{d}^{i, j}$ with $0<\kappa_{2}^{i, j} \leq \ldots \leq \kappa_{d}^{i, j}$ and we let $\left\{\mathbf{u}_{2}^{i, j}, \ldots, \mathbf{u}_{d}^{i, j}\right\}$ be a basis of $\mathbb{R}^{d-1}$, consisting of corresponding eigenvectors. Based on these vectors we put $U_{i, j}:=\left(\mathbf{u}_{2}^{i, j}|\ldots| \mathbf{u}_{d}^{i, j}\right)$. These definitions yield $H_{i, j} \mathbf{u}_{k}^{i, j}=\kappa_{k}^{i, j} \mathbf{u}_{k}^{i, j}$ for $k \in\{2, \ldots, d\}$, 


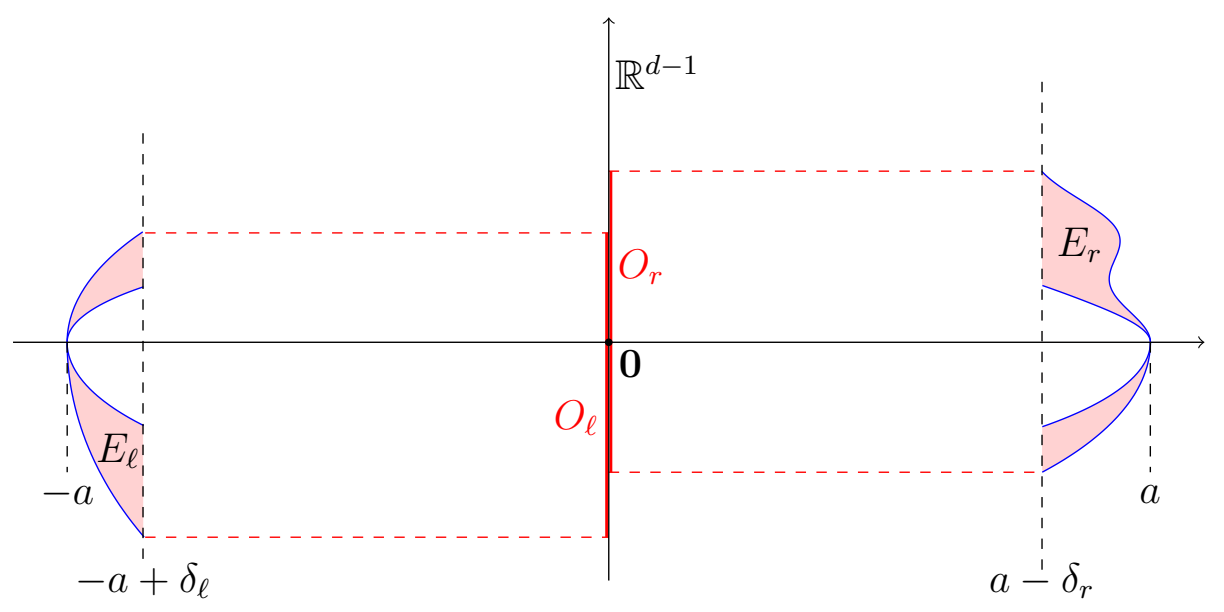

Figure 5.28: The setting under Condition 1 and Condition 7.

$U_{i, j} U_{i, j}^{\top}=\mathrm{I}_{d-1}$, and $U_{i, j}^{\top} H_{i, j} U_{i, j}=\operatorname{diag}\left(\kappa_{2}^{i, j}, \ldots, \kappa_{d}^{i, j}\right)=: D_{i, j}$. With this notation, Condition 3 reads as follows:

Condition 8. For some constant $\eta \in(0,1)$, the $2(d-1) \times 2(d-1)$-dimensional matrix

$$
A(\eta):=\left(\begin{array}{cc}
2 a \eta D_{\ell, 1}-\mathrm{I}_{d-1} & U_{\ell, 1}^{\top} U_{r, 1} \\
U_{r, 1}^{\top} U_{\ell, 1} & 2 a \eta D_{r, 1}-\mathrm{I}_{d-1}
\end{array}\right)
$$

is positive semi-definite.

Observe that the only difference between Condition 8 and Condition 3 lies in the more lengthy notation. At this point we have to define the new limiting sets for the point processes. For $i \in\{\ell, r\}$ we put

$$
P\left(H_{i, 1}, H_{i, 2}\right):=\left\{\left(z_{1}, \widetilde{z}\right) \in \mathbb{R}^{d}: \frac{1}{2} \widetilde{z}^{\top} H_{i, 1} \widetilde{z} \leq z_{1} \leq \frac{1}{2} \widetilde{z}^{\top} H_{i, 2} \widetilde{z}\right\} .
$$

We have to ensure that these limiting sets are not lower-dimensional, i.e. the case

$$
m_{d}\left(P\left(H_{i, 1}, H_{i, 2}\right)\right)=0
$$

has to be excluded. Otherwise, the limiting point processes would degenerate into point processes with 0 points almost surely.

Condition 9. There are indices $i, j \in\{2, \ldots, d\}$ so that

$$
\kappa_{i}^{\ell, 1}<\kappa_{i}^{\ell, 2} \quad \text { and } \quad \kappa_{j}^{r, 1}<\kappa_{j}^{r, 2} .
$$

We again consider distributions $\mathbb{P}_{Z}$ with a Lebesgue density $f$ supported by $E$ that is continuous and bounded away from 0 at the poles. Condition 4 can be left completely 
unchanged compared to Chapter 3. Now we can state the limiting result under this setting:

Theorem 5.13. If Conditions 1, 7, 8, 9 and 4 hold, then

$$
n^{\frac{2}{d+1}}\left(2 a-\operatorname{diam}\left(\mathbf{Z}_{n}\right)\right) \stackrel{\mathcal{D}}{\longrightarrow} \min _{i, j \geq 1}\left\{\mathcal{X}_{i, 1}+\mathcal{Y}_{j, 1}-\frac{1}{4 a}\left|\widetilde{\mathcal{X}}_{i}-\widetilde{\mathcal{Y}}_{j}\right|^{2}\right\}
$$

where $\left\{\mathcal{X}_{i}, i \geq 1\right\} \stackrel{\mathcal{D}}{=} \operatorname{PRM}\left(\left.p_{\ell} \cdot m_{d}\right|_{P\left(H_{\ell, 1}, H_{\ell, 2}\right)}\right) \quad$ and $\left\{\mathcal{Y}_{j}, j \geq 1\right\} \stackrel{\mathcal{D}}{=} \operatorname{PRM}\left(p_{r}\right.$ $\left.\left.m_{d}\right|_{P\left(H_{r, 1}, H_{r, 2}\right)}\right)$ are independent Poisson processes. The same holds true if we replace $\operatorname{diam}\left(\mathbf{Z}_{n}\right)$ with $M_{n}$.

Proof. The one and only change compared to the proof of Theorem 3.5 occurs in the proof of Lemma 4.6. We shift the set $E_{\ell}$ to the right by $a \cdot \mathbf{e}_{1}$ along the $z_{1}$-axis and call this set $P_{1}\left(H_{\ell, 1}, H_{\ell, 2}\right)$. The set $E_{r}$ gets translated by $-a \cdot \mathbf{e}_{1}$ to the left along the $z_{1}$-axis and is then reflected at the plane $\left\{z_{1}=0\right\}$. We call the resulting set $P_{1}\left(H_{r, 1}, H_{r, 2}\right)$ and obtain the representation

$$
\begin{aligned}
& P_{1}\left(H_{i, 1}, H_{i, 2}\right)= \\
& \quad\left\{\left(z_{1}, \widetilde{z}\right) \in \mathbb{R}^{d}: \frac{1}{2} \widetilde{z}^{\top} H_{i, 1} \widetilde{z}+o\left(|\widetilde{z}|^{2}\right) \leq z_{1} \leq \frac{1}{2} \widetilde{z}^{\top} H_{i, 2} \widetilde{z}+o\left(|\widetilde{z}|^{2}\right), z_{1}<\delta_{i}, \widetilde{z} \in O_{i}\right\}
\end{aligned}
$$

for $i \in\{\ell, r\}$. The constant $\delta^{*}$ is chosen in such a way, that the assertion of a correspondingly adjusted version of Lemma 4.3 holds true for each $\delta \leq \delta^{*}$. With $P_{n}\left(H_{i, 1}, H_{i, 2}\right):=T_{n}\left(P_{1}\left(H_{i, 1}, H_{i, 2}\right) \cap\left\{z_{1} \leq \delta^{*}\right\}\right)$ for $i \in\{\ell, r\}$ and $n \in \mathbb{N}$ we get

$$
\begin{aligned}
& P_{n}\left(H_{i, 1}, H_{i, 2}\right) \\
= & \left\{z \in \mathbb{R}^{d}: T_{n}^{-1}(z) \in P_{1}\left(H_{i, 1}, H_{i, 2}\right) \cap\left\{z_{1} \leq \delta^{*}\right\}\right\} \\
= & \left\{z \in \mathbb{R}^{d}: \frac{1}{2}\left(\frac{1}{n^{\nu}} \widetilde{z}\right)^{\top} H_{i, 1}\left(\frac{1}{n^{\nu}} \widetilde{z}\right)+o\left(\left|\frac{1}{n^{\nu}} \widetilde{z}\right|^{2}\right) \leq \frac{z_{1}}{n^{2 \nu}}\right. \\
\quad \leq & \left.\frac{1}{2}\left(\frac{1}{n^{\nu}} \widetilde{z}\right)^{\top} H_{i, 2}\left(\frac{1}{n^{\nu}} \widetilde{z}\right)+o\left(\left|\frac{1}{n^{\nu}} \widetilde{z}\right|^{2}\right), \frac{z_{1}}{n^{2 \nu}}<\delta^{*}, \frac{1}{n^{\nu}} \widetilde{z} \in O_{i}\right\} \\
= & \left\{z \in \mathbb{R}^{d}: \frac{1}{2} \widetilde{z}^{\top} H_{i, 1} \widetilde{z}+o\left(|\widetilde{z}|^{2}\right) \leq z_{1} \leq \frac{1}{2} \widetilde{z}^{\top} H_{i, 2} \widetilde{z}+o\left(|\widetilde{z}|^{2}\right), z_{1}<n^{2 \nu} \delta^{*}, \widetilde{z} \in n^{\nu} O_{i}\right\}
\end{aligned}
$$

and hence $\mathbb{1}\left\{z \in P_{n}\left(H_{i, 1}, H_{i, 2}\right)\right\} \rightarrow \mathbb{1}\left\{z \in P\left(H_{i, 1}, H_{i, 2}\right)\right\}$ for almost all $z \in \mathbb{R}^{d}$.

Example 5.14. An easy example for a set covered by Conditions 1,7 and 8 is given 
if we put $d=2$ and define the non-convex but closed (difference) set

$$
E:=\left\{z \in \mathbb{R}^{2}:\left(\frac{z_{1}}{a_{1}}\right)^{2}+\left(\frac{z_{2}}{a_{2,1}}\right)^{2} \leq 1\right\} \backslash\left\{z \in \mathbb{R}^{2}:\left(\frac{z_{1}}{a_{1}}\right)^{2}+\left(\frac{z_{2}}{a_{2,2}}\right)^{2}<1\right\}
$$

where $a_{1}>a_{2,1}>a_{2,2}>0$. As in Remark 3.6 we obtain $\kappa_{2}^{\ell, 1}=\kappa_{2}^{r, 1}=a_{1} / a_{2,1}^{2}$, $\kappa_{2}^{\ell, 2}=\kappa_{2}^{r, 2}=a_{1} / a_{2,2}^{2}$ and hence

$$
H_{\ell, j}=H_{r, j}=\left(\frac{a_{1}}{a_{2, j}^{2}}\right)
$$

$j \in\{1,2\}$. So, the limiting sets are given by

$$
P\left(H_{\ell, 1}, H_{\ell, 2}\right)=P\left(H_{r, 1}, H_{r, 2}\right)=\left\{z \in \mathbb{R}^{2}: \frac{z_{2}^{2}}{a_{2,1}^{2}} \leq \frac{2 z_{1}}{a_{1}} \leq \frac{z_{2}^{2}}{a_{2,2}^{2}}\right\} .
$$

Figure 5.29 shows the sets $E$ (left) and $P\left(H_{\ell, 1}, H_{\ell, 2}\right)=P\left(H_{r, 1}, H_{r, 2}\right)$ (right) for $a_{1}=1, a_{2,1}=1 / 2$ and $a_{2,2}=1 / 4$. Notice the different scalings between the left-hand and the right-hand image.
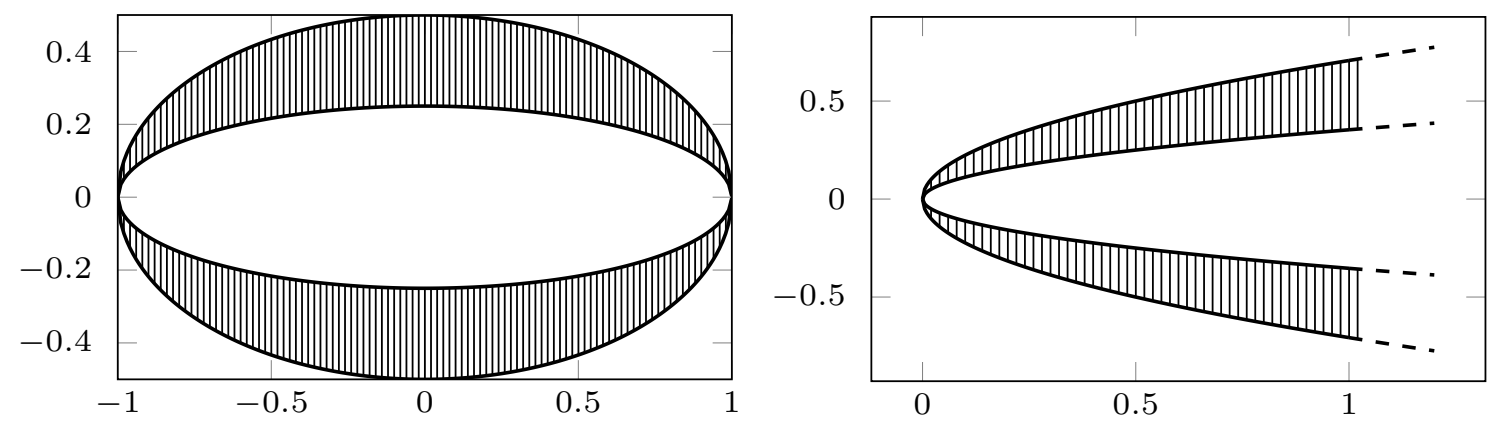

Figure 5.29: The sets $E$ (left) and $P\left(H_{\ell, 1}, H_{\ell, 2}\right)=P\left(H_{r, 1}, H_{r, 2}\right)$ (right) for $a_{1}=$ $1, a_{2,1}=1 / 2$ and $a_{2,2}=1 / 4$.

\section{$5.5 p$-SUPERELLIPSOIDS AND $p$-NORMS}

\subsubsection{Conditions AND MAIN RESUlts}

For $1 \leq p<\infty$ and $a_{1}>a_{2} \geq a_{3} \geq \ldots \geq a_{d}>0$ we define the so-called $p$-superellipsoid

$$
E^{p}:=\left\{z \in \mathbb{R}^{d}: \sum_{k=1}^{d}\left(\frac{\left|z_{k}\right|}{a_{k}}\right)^{p} \leq 1\right\}
$$


and the corresponding $p$-norm

$$
|z|_{p}:=\left(\sum_{k=1}^{d}\left|z_{k}\right|^{p}\right)^{\frac{1}{p}}, \quad z \in \mathbb{R}^{d} .
$$

Moreover, based on this norm, let

$$
\operatorname{diam}_{p}(A):=\sup _{x, y \in A}|x-y|_{p}
$$

be the so-called $p$-diameter of a set $A \subset \mathbb{R}^{d}$. The definitions of $E^{p}$ and $|\cdot|_{p}$ yield $\left|\left(-a_{1}, \mathbf{0}\right)-\left(a_{1}, \mathbf{0}\right)\right|_{p}=2 a_{1}$, and in view of $a_{1}>a_{2} \geq a_{3} \geq \ldots \geq a_{d}>0$ we have $|z|_{p} \leq a_{1}$ for each $z \in E^{p}$, with equality only for $z \in\left\{\left(-a_{1}, \mathbf{0}\right),\left(a_{1}, \mathbf{0}\right)\right\}$. Together with $|x-y|_{p} \leq|x|_{p}+|y|_{p}$ for all $x, y \in \mathbb{R}^{d}$ we can infer that the set $E^{p}$ has a unique diameter of length $2 a_{1}$ with respect to the $p$-norm between the points $\left(-a_{1}, \mathbf{0}\right)$ and $\left(a_{1}, \mathbf{0}\right)$.

We assume that the random variables $Z_{1}, Z_{2}, \ldots$ are i.i.d. with a common density $f$, supported by the superellipsoid $E^{p}$. As in Chapter 3, we consider densities that are continuous and bounded away from 0 at the poles. In this section we will investigate the largest distance between these random points with respect to the corresponding $p$-norm, not with respect to the Euclidean norm, i.e. we consider

$$
M_{n}^{p}:=\max _{1 \leq i, j \leq n}\left|Z_{i}-Z_{j}\right|_{p}
$$

We recall the definition

$$
\mathbf{Z}_{n}:=\sum_{i=1}^{N_{n}} \varepsilon_{Z_{i}}
$$

where $N_{n}$ is independent of $Z_{1}, Z_{2}, \ldots$ and has a Poisson distribution with parameter $n$. $\mathbf{Z}_{n}$ is a Poisson process in $\mathbb{R}^{d}$ with intensity measure $n \mathbb{P}_{Z}$, and we get

$$
\operatorname{diam}_{p}\left(\mathbf{Z}_{n}\right)=\max _{1 \leq i, j \leq N_{n}}\left|Z_{i}-Z_{j}\right|_{p}
$$

With the new limiting set

$$
P^{p}:=\left\{z \in \mathbb{R}^{d}: \sum_{k=2}^{d}\left(\frac{\left|z_{k}\right|}{a_{k}}\right)^{p} \leq \frac{p z_{1}}{a_{1}}\right\},
$$

we can state our result for this setting: 
Theorem 5.15. Under the standing assumptions of this section and if Condition 4 holds true for $E$ replaced with $E^{p}$ and $a=a_{1}$, then

$$
n^{\frac{p}{d+p-1}}\left(2 a_{1}-\operatorname{diam}_{p}\left(\mathbf{Z}_{n}\right)\right) \stackrel{\mathcal{D}}{\longrightarrow} \min _{i, j \geq 1}\left\{\mathcal{X}_{i, 1}+\mathcal{Y}_{j, 1}-\frac{1}{p\left(2 a_{1}\right)^{p-1}}\left|\widetilde{\mathcal{X}}_{i}-\widetilde{\mathcal{Y}}_{j}\right|_{p}^{p}\right\}
$$

where $\left\{\mathcal{X}_{i}, i \geq 1\right\} \stackrel{\mathcal{D}}{=} \operatorname{PRM}\left(\left.p_{\ell} \cdot m_{d}\right|_{P^{p}}\right)$ and $\left\{\mathcal{Y}_{j}, j \geq 1\right\} \stackrel{\mathcal{D}}{=} \operatorname{PRM}\left(\left.p_{r} \cdot m_{d}\right|_{P^{p}}\right)$ are independent Poisson processes. The same holds true if we replace $\operatorname{diam}_{p}\left(\mathbf{Z}_{n}\right)$ with $M_{n}^{p}$.

The proof of this theorem can be found after the following corollary and the corresponding plots.

Corollary 5.16. Given the uniform distribution on $E^{p}$, Condition 4 holds true for $E$ replaced with $E^{p}, a=a_{1}$ and

$$
p_{\ell}=p_{r}=\frac{1}{m_{d}\left(E^{p}\right)}=\left(\frac{\left(2 \Gamma\left(1+\frac{1}{p}\right)\right)^{d} \prod_{i=1}^{d} a_{i}}{\Gamma\left(1+\frac{d}{p}\right)}\right)^{-1}>0
$$

see Wang [25]. We can thus apply Theorem 5.15. For $d=2, a_{1}=1, a_{2}=1 / 2$ and $p \in\{1,3 / 2,4\}$, the sets $E^{p}$ and $P^{p}$ and the results of a simulation study are illustrated in the Figures 5.30 to 5.35. Notice that Corollary 3.7 is a special case of this corollary, namely for $p=2$.
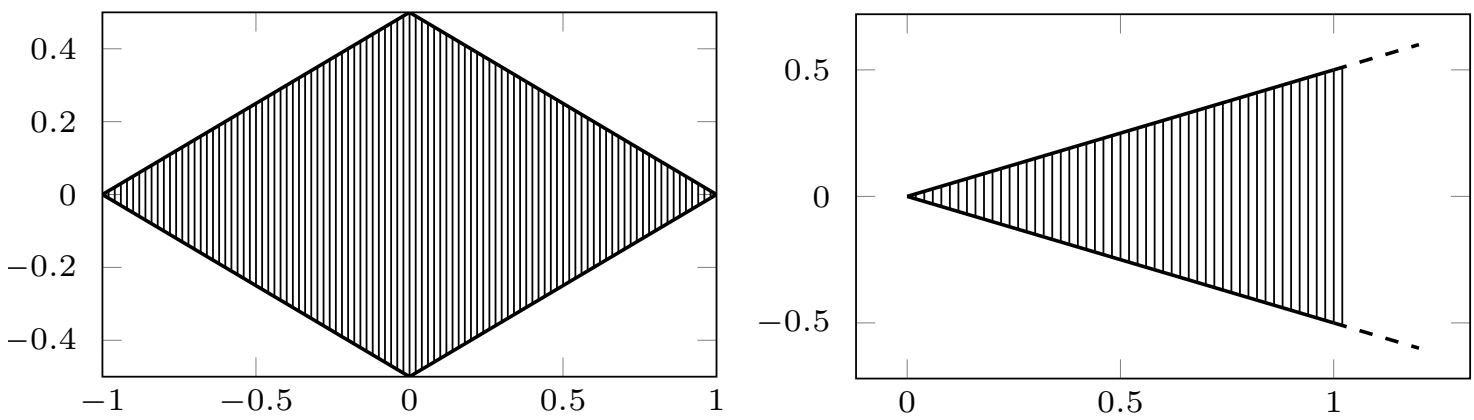

Figure 5.30: The sets $E^{p}$ (left) and $P^{p}$ (right) for $d=2$ with $p=1, a_{1}=1, a_{2}=1 / 2$.

Since the proof of Theorem 5.15 can be done by using the same techniques as seen in the proof of Theorem 3.5, we will only show the crucial differences. In a first subsection, we will focus on the behavior of the general $p$-norm of the difference of two points, lying close to the two poles. In a second subsection, we investigate the shape of the superellipsoid $E^{p}$ close to the poles. The third and last subsection will point out the few differences in the main part of the proof compared to that of Theorem 3.5 in Section 4.3. 


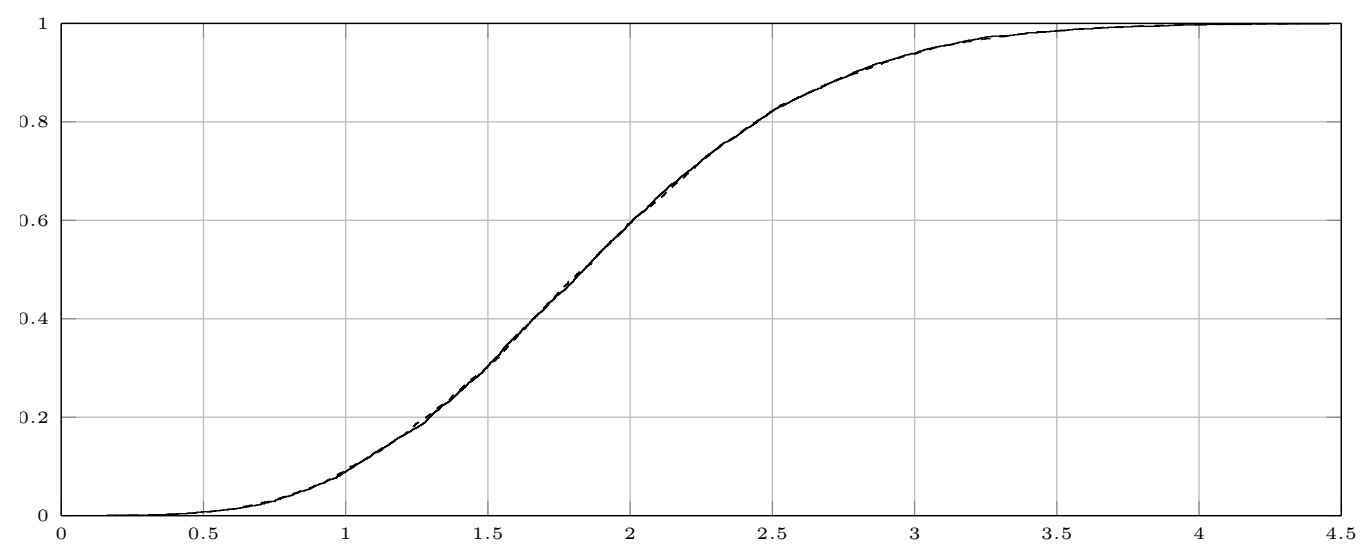

Figure 5.31: Empirical distribution function of $n^{1 / 2}\left(2-M_{n}^{p}\right)$ in the setting of Corollary 5.16 for $d=2$ with $p=1, a_{1}=1, a_{2}=1 / 2, n=1000$ (solid, 5000 replications). The limit distribution is approximated as described after Corollary 3.7 with $b=10$ (dashed, 5000 replications).
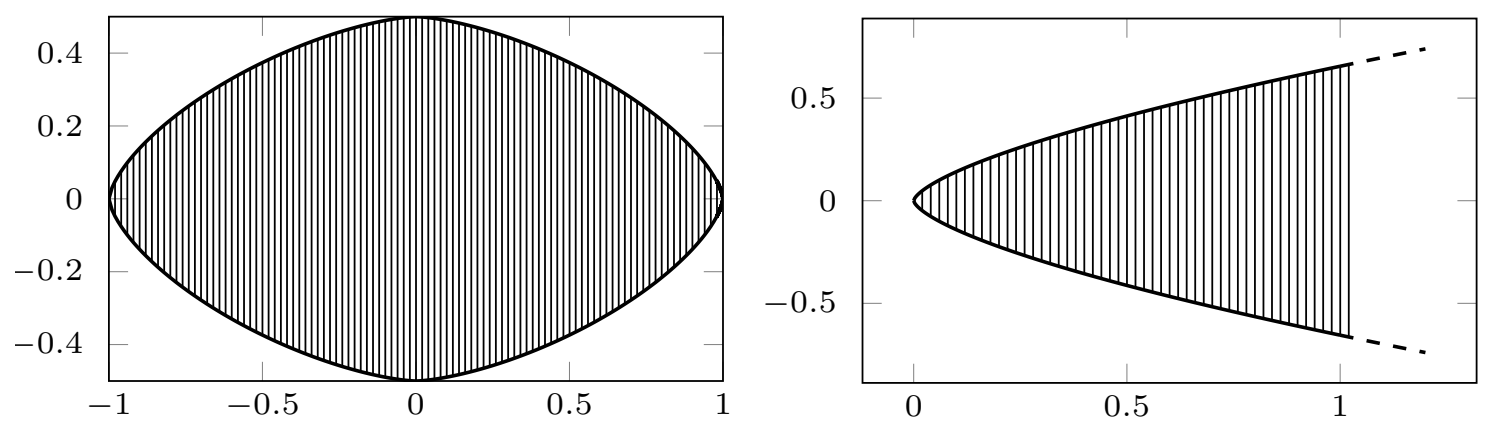

Figure 5.32: The sets $E^{p}$ (left) and $P^{p}$ (right) for $d=2$ with $p=3 / 2, a_{1}=1, a_{2}=1 / 2$.

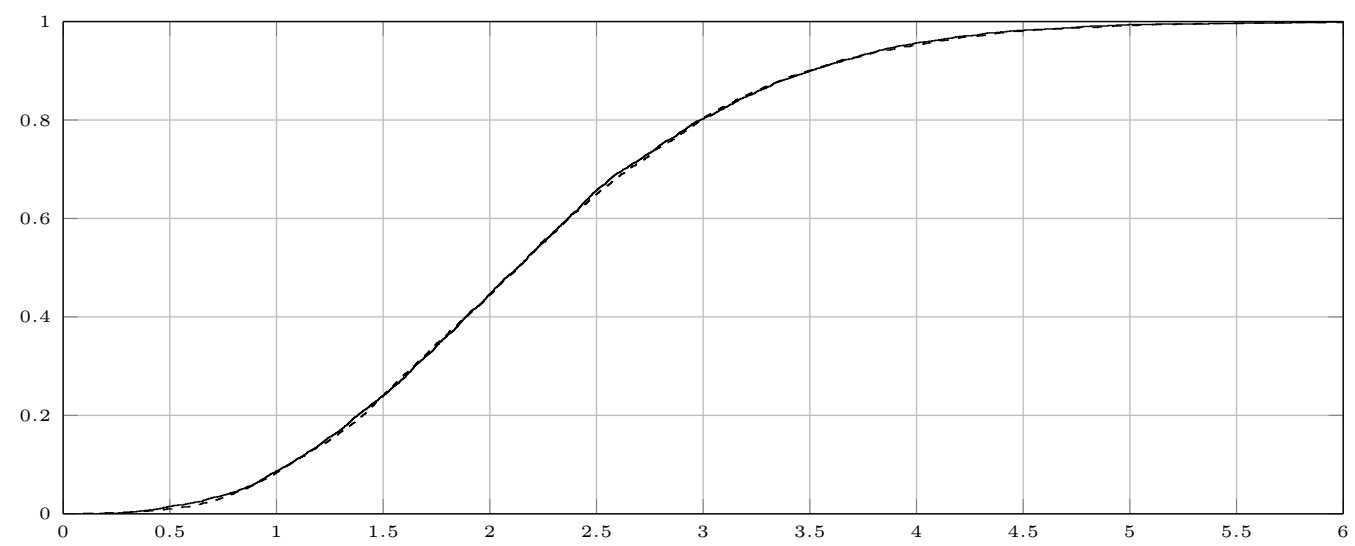

Figure 5.33: Empirical distribution function of $n^{3 / 5}\left(2-M_{n}^{p}\right)$ in the setting of Corollary 5.16 for $d=2$ with $p=3 / 2, a_{1}=1, a_{2}=1 / 2, n=1000$ (solid, 5000 replications). The limit distribution is approximated as described after Corollary 3.7 with $b=10$ (dashed, 5000 replications). 

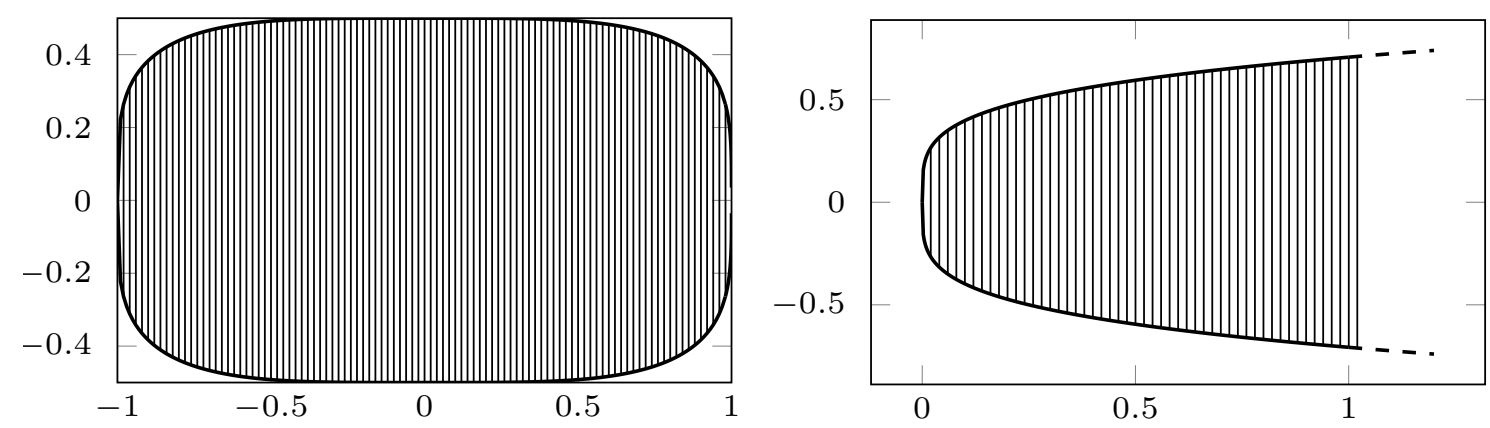

Figure 5.34: The sets $E^{p}$ (left) and $P^{p}$ (right) for $d=2$ with $p=4, a_{1}=1, a_{2}=1 / 2$.

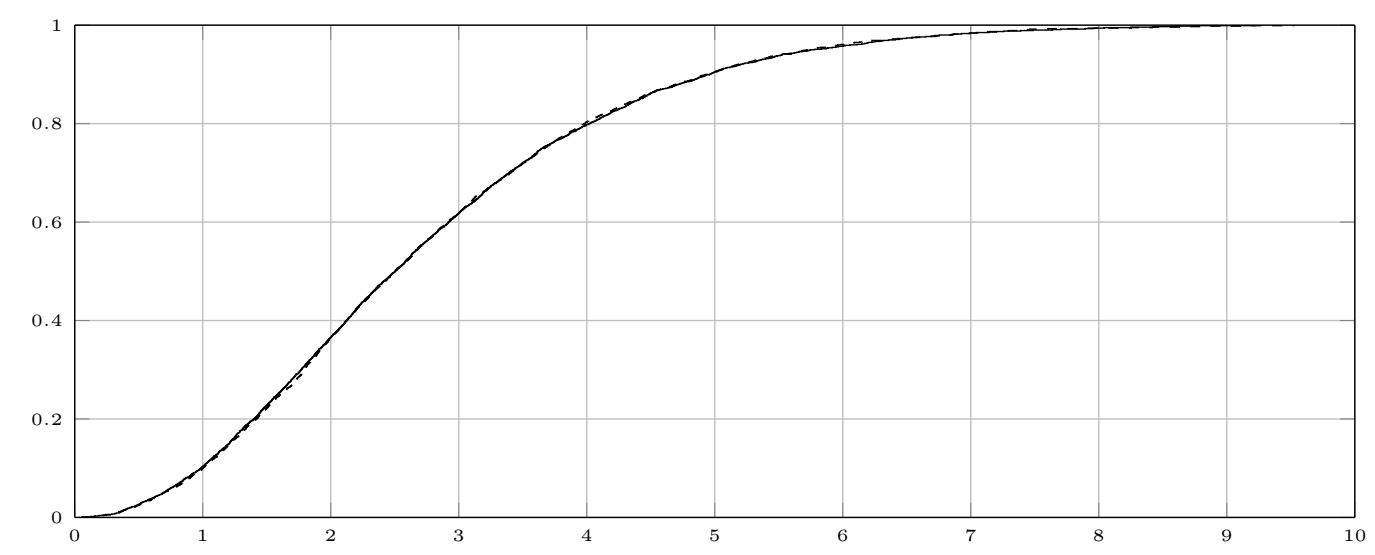

Figure 5.35: Empirical distribution function of $n^{4 / 5}\left(2-M_{n}^{p}\right)$ in the setting of Corollary 5.16 for $d=2$ with $p=4, a_{1}=1, a_{2}=1 / 2, n=1000$ (solid, 5000 replications). The limit distribution is approximated as described after Corollary 3.7 with $b=10$ (dashed, 5000 replications).

\subsubsection{The Behavior of $|x-y|_{p}$ Close to the Poles}

For the proof of Theorem 3.5 we used a $2 d$-dimensional Taylor series expansion of $|x-y|$ for $x$ close to $(-a, \mathbf{0})$ and $y$ close to $(a, \mathbf{0})$, see $(4.8)$. But, for general $p \in[1, \infty)$, the function $(x, y) \mapsto|x-y|_{p}$ is not differentiable at the point $(x, y)=$ $\left(-a_{1}, \mathbf{0}, a_{1}, \mathbf{0}\right) \in \mathbb{R}^{2 d}$ due to the absolute values (that were no problem in the case $p=2)$. Being more precise, this function does not have partial derivatives at the point $\left(-a_{1}, \mathbf{0}, a_{1}, \mathbf{0}\right)$ with respect to the components $x_{2}, \ldots, x_{d}, y_{2}, \ldots, y_{d}$. For $x_{1}$ and $y_{1}$, the partial derivatives exist, since we have $\left|x_{1}-y_{1}\right|^{p}=\left(y_{1}-x_{1}\right)^{p}$ for $\left(x_{1}, y_{1}\right)$ close to $\left(-a_{1}, a_{1}\right)$. Without stressing the dependence on the underlying dimension, we will also use the notation $|\cdot|_{p}$ for the $p$-norm on $\mathbb{R}^{d-1}$. Defining

$$
s:=|\widetilde{x}-\widetilde{y}|_{p}^{p}
$$

we obtain

$$
|x-y|_{p}=\left(\left(y_{1}-x_{1}\right)^{p}+s\right)^{\frac{1}{p}}
$$


and instead of $(x, y) \rightarrow\left(-a_{1}, \mathbf{0}, a_{1}, \mathbf{0}\right)$ we consider $\left(x_{1}, y_{1}, s\right) \rightarrow\left(-a_{1}, a_{1}, 0\right)$. Observe that for points lying in $E^{p}$ the convergence $\left(x_{1}, y_{1}\right) \rightarrow\left(-a_{1}, a_{1}\right)$ implies $s \rightarrow 0$. A three-dimensional Taylor series expansion at the point $\mathbf{a}:=\left(-a_{1}, a_{1}, 0\right)$ gives

$$
|x-y|_{p}=\left(\left(y_{1}-x_{1}\right)^{p}+s\right)^{\frac{1}{p}}=-x_{1}+y_{1}+\frac{s}{p\left(2 a_{1}\right)^{p-1}}+R\left(x_{1}, y_{1}, s\right),
$$

where $R\left(x_{1}, y_{1}, t\right)=o\left(\left|\left(x_{1}, y_{1}, t\right)-\mathbf{a}\right|\right)$ as $r \rightarrow 0$, uniformly on the (three-dimensional) ball $B_{r}(\mathbf{a})$ of radius $r$ and center $\mathbf{a}$. This uniform convergence especially holds on

$$
B_{r}^{*}(\mathbf{a}):=\left\{\left(x_{1}, y_{1}, t\right) \in B_{r}(\mathbf{a}):-a_{1}<x_{1}, y_{1}<a_{1}\right\}
$$

as $r \rightarrow 0$. Since we will only consider points lying in $E^{p}$, it will be sufficient to merely use this subset of $B_{r}(\mathbf{a})$. Putting

$$
\bar{G}\left(x_{1}, y_{1}, t\right):=\left(a_{1}+x_{1}\right)+\left(a_{1}-y_{1}\right)-\frac{t}{p\left(2 a_{1}\right)^{p-1}},
$$

we obtain

$$
2 a_{1}-|x-y|_{p}=\bar{G}\left(x_{1}, y_{1}, s\right)-R\left(x_{1}, y_{1}, s\right) .
$$

In Lemma 5.20 we will demonstrate that $R\left(x_{1}, y_{1}, s\right)$ is asymptotically negligible in comparison to $\bar{G}\left(x_{1}, y_{1}, s\right)$ as $(x, y) \rightarrow\left(-a_{1}, \mathbf{0}, a_{1}, \mathbf{0}\right) \in \mathbb{R}^{2 d}$ inside of $E^{p} \times E^{p}$. Before we can show this asymptotical behavior, we need two additional lemmata.

Lemma 5.17. For any $z \in E^{p}$ we have

$$
|\widetilde{z}|_{p} \leq \frac{a_{2}}{a_{1}}\left(a_{1}^{p}-\left|z_{1}\right|^{p}\right)^{\frac{1}{p}}
$$

Proof. Using $z \in E^{p}$ and $a_{2} \geq a_{3} \geq \ldots \geq a_{d}$ yields

$$
\begin{array}{rlrl} 
& & \left(\frac{\left|z_{1}\right|}{a_{1}}\right)^{p}+\sum_{k=2}^{d}\left(\frac{\left|z_{k}\right|}{a_{k}}\right)^{p} & \leq 1 \\
\Longrightarrow & \left(\frac{\left|z_{1}\right|}{a_{1}}\right)^{p}+\frac{1}{a_{2}^{p}} \sum_{k=2}^{d}\left|z_{k}\right|^{p} & \leq 1 \\
\Longrightarrow & |\widetilde{z}|_{p}^{p} \leq a_{2}^{p}\left(1-\left(\frac{\left|z_{1}\right|}{a_{1}}\right)^{p}\right) \\
\Longrightarrow & |\widetilde{z}|_{p} \leq a_{2}\left(1-\left(\frac{\left|z_{1}\right|}{a_{1}}\right)^{p}\right)^{\frac{1}{p}} \\
\Longrightarrow & |\widetilde{z}|_{p} \leq \frac{a_{2}}{a_{1}}\left(a_{1}^{p}-\left|z_{1}\right|^{p}\right)^{\frac{1}{p}} .
\end{array}
$$


We define $E_{\ell}^{p}:=E^{p} \cap\left\{z_{1}<0\right\}$ and $E_{r}^{p}:=E^{p} \cap\left\{z_{1}>0\right\}$. For $(x, y) \in E_{\ell}^{p} \times E_{r}^{p}$ with both $\left|-a_{1}-x_{1}\right|$ and $\left|a_{1}-y_{1}\right|$ 'small', it is quite obvious that the value $s$ has to be 'small', too. For being more precise, we fix $\delta \in\left(0, a_{1}\right)$ and define

$$
E_{\delta}^{p}:=\left\{(x, y) \in E_{\ell}^{p} \times E_{r}^{p}:-a_{1} \leq x_{1} \leq-a_{1}+\delta, a_{1}-\delta \leq y_{1} \leq a_{1}\right\}
$$

We then can show the following lemma:

Lemma 5.18. If $\delta \in\left(0, a_{1}\right)$ and $(x, y) \in E_{\delta}^{p}$ with $\left|x_{1}\right| \leq\left|y_{1}\right|<a_{1}$, we have

$$
s \leq\left(a_{1}+x_{1}\right) \cdot\left(\frac{a_{2}}{a_{1}}\right)^{p} \cdot p \cdot\left(2 a_{1}\right)^{p-1} \cdot\left(1+\frac{a_{1}-y_{1}}{a_{1}+x_{1}}\right),
$$

and hence especially $s \leq\left(a_{1}+x_{1}\right) \cdot \frac{p\left(2 a_{2}\right)^{p}}{a_{1}}$ and $s \leq \delta \cdot \frac{p\left(2 a_{2}\right)^{p}}{a_{1}}$.

Proof. The triangle inequality and Lemma 5.17 give

$$
\begin{aligned}
s & =|\widetilde{x}-\widetilde{y}|_{p}^{p} \\
& \leq\left(|\widetilde{x}|_{p}+|\widetilde{y}|_{p}\right)^{p} \\
& \leq\left(\frac{a_{2}}{a_{1}}\left(a_{1}^{p}-\left|x_{1}\right|^{p}\right)^{\frac{1}{p}}+\frac{a_{2}}{a_{1}}\left(a_{1}^{p}-\left|y_{1}\right|^{p}\right)^{\frac{1}{p}}\right)^{p} \\
& =\left(\frac{a_{2}}{a_{1}}\right)^{p} \cdot\left(a_{1}+x_{1}\right) \cdot\left(\left(\frac{a_{1}^{p}-\left|x_{1}\right|^{p}}{a_{1}+x_{1}}\right)^{\frac{1}{p}}+\left(\frac{a_{1}^{p}-\left|y_{1}\right|^{p}}{a_{1}+x_{1}}\right)^{\frac{1}{p}}\right)^{p} \\
& =\left(\frac{a_{2}}{a_{1}}\right)^{p} \cdot\left(a_{1}+x_{1}\right) \cdot\left(\left(\frac{a_{1}^{p}-\left|x_{1}\right|^{p}}{a_{1}+x_{1}}\right)^{\frac{1}{p}}+\left(\frac{a_{1}-y_{1}}{a_{1}+x_{1}}\right)^{\frac{1}{p}}\left(\frac{a_{1}^{p}-\left|y_{1}\right|^{p}}{a_{1}-y_{1}}\right)^{\frac{1}{p}}\right)^{p} .
\end{aligned}
$$

Since $y_{1}>0$, we have

$$
\frac{a_{1}^{p}-\left|y_{1}\right|^{p}}{a_{1}-y_{1}}=\frac{a_{1}^{p}-y_{1}^{p}}{a_{1}-y_{1}}
$$

which is the slope of the line joining the points $\left(y_{1}, y_{1}^{p}\right)$ and $\left(a_{1}, a_{1}^{p}\right)$. Using the mean value theorem justifies the existence of some $t_{0} \in\left(y_{1}, a_{1}\right)$ with

$$
\frac{a_{1}^{p}-y_{1}^{p}}{a_{1}-y_{1}}=p \cdot t_{0}^{p-1} \leq p \cdot a_{1}^{p-1}
$$

For the inequality, notice that $p \geq 1$, i.e. $t \mapsto t^{p-1}$ is monotonically increasing on $\left(y_{1}, a_{1}\right)$. A symmetry argument shows that $\frac{a_{1}^{p}-\left|x_{1}\right|^{p}}{a_{1}+x_{1}}$ is bounded from above on $\left(-a_{1}, 0\right]$ by the same constant. The monotonicity of the function $t \mapsto t^{\frac{1}{p}}$ gives

$$
\sup _{x_{1} \in\left(-a_{1}, 0\right]}\left(\frac{a_{1}^{p}-\left|x_{1}\right|^{p}}{a_{1}+x_{1}}\right)^{\frac{1}{p}}=\sup _{y_{1} \in\left[0, a_{1}\right)}\left(\frac{a_{1}^{p}-\left|y_{1}\right|^{p}}{a_{1}-y_{1}}\right)^{\frac{1}{p}}=\left(p \cdot a_{1}^{p-1}\right)^{\frac{1}{p}}=p^{\frac{1}{p}} \cdot a_{1}^{\frac{p-1}{p}} .
$$


These considerations imply

$$
\begin{aligned}
s & \leq\left(\frac{a_{2}}{a_{1}}\right)^{p} \cdot\left(a_{1}+x_{1}\right) \cdot\left(p^{\frac{1}{p}} \cdot a_{1}^{\frac{p-1}{p}}+\left(\frac{a_{1}-y_{1}}{a_{1}+x_{1}}\right)^{\frac{1}{p}} p^{\frac{1}{p}} \cdot a_{1}^{\frac{p-1}{p}}\right)^{p} \\
& =\left(a_{1}+x_{1}\right) \cdot\left(\frac{a_{2}}{a_{1}}\right)^{p} \cdot p \cdot a_{1}^{p-1} \cdot\left(1+\left(\frac{a_{1}-y_{1}}{a_{1}+x_{1}}\right)^{\frac{1}{p}}\right)^{p} .
\end{aligned}
$$

The convexity of the function $t \mapsto t^{p}$ leads to

$$
\left(1+t^{\frac{1}{p}}\right)^{p}=2^{p} \cdot\left(\frac{1}{2} \cdot 1+\frac{1}{2} \cdot t^{\frac{1}{p}}\right)^{p} \leq 2^{p} \cdot\left(\frac{1}{2} \cdot 1+\frac{1}{2} \cdot t\right)=2^{p-1} \cdot(1+t)
$$

and hence

$$
s \leq\left(a_{1}+x_{1}\right) \cdot\left(\frac{a_{2}}{a_{1}}\right)^{p} \cdot p \cdot a_{1}^{p-1} \cdot 2^{p-1} \cdot\left(1+\frac{a_{1}-y_{1}}{a_{1}+x_{1}}\right) .
$$

Under the assumptions $(x, y) \in E_{\delta}^{p}$ and $\left|x_{1}\right| \leq\left|y_{1}\right|<a_{1}$ we have $0<a_{1}-y_{1} \leq a_{1}+x_{1}$ and $0<\frac{a_{1}-y_{1}}{a_{1}+x_{1}} \leq 1$. So, we obtain

$$
s \leq\left(a_{1}+x_{1}\right) \cdot\left(\frac{a_{2}}{a_{1}}\right)^{p} \cdot p \cdot a_{1}^{p-1} \cdot 2^{p}=\left(a_{1}+x_{1}\right) \cdot \frac{p\left(2 a_{2}\right)^{p}}{a_{1}},
$$

and from $-a_{1} \leq x_{1} \leq-a_{1}+\delta$ we finally get

$$
s \leq \delta \cdot \frac{p\left(2 a_{2}\right)^{p}}{a_{1}}
$$

The following corollary is an important implication of this lemma:

Corollary 5.19. For each $r>0$, we can find $\delta>0$ sufficiently small, so that, for all $(x, y) \in E_{\delta}^{p}$, we have $\left(x_{1}, y_{1}, s\right) \in B_{r}^{*}(\mathbf{a})$.

This corollary justifies the consideration of $\left(x_{1}, y_{1}, t\right) \rightarrow \mathbf{a}$ instead of $(x, y) \rightarrow$ $\left(-a_{1}, \mathbf{0}, a_{1}, \mathbf{0}\right)$. Now we can state an adapted version of Lemma 4.5:

Lemma 5.20. We have $R\left(x_{1}, y_{1}, s\right)=o\left(\bar{G}\left(x_{1}, y_{1}, s\right)\right)$ as $\delta \rightarrow 0$, uniformly on $E_{\delta}^{p}$.

Proof. Notice that

$$
\frac{R\left(x_{1}, y_{1}, t\right)}{\bar{G}\left(x_{1}, y_{1}, t\right)}=\frac{R\left(x_{1}, y_{1}, t\right)}{\left|\left(x_{1}, y_{1}, t\right)-\mathbf{a}\right|} \cdot \frac{\left|\left(x_{1}, y_{1}, t\right)-\mathbf{a}\right|}{\bar{G}\left(x_{1}, y_{1}, t\right)}=o(1) \frac{\left|\left(x_{1}, y_{1}, t\right)-\mathbf{a}\right|}{\bar{G}\left(x_{1}, y_{1}, t\right)}
$$


as $r \rightarrow 0$, where $o(1)$ is uniformly on $B_{r}^{*}(\mathbf{a})$. From Corollary 5.19 we conclude

$$
\frac{R\left(x_{1}, y_{1}, s\right)}{\bar{G}\left(x_{1}, y_{1}, s\right)}=o(1) \frac{\left|\left(x_{1}, y_{1}, s\right)-\mathbf{a}\right|}{\bar{G}\left(x_{1}, y_{1}, s\right)}
$$

as $\delta \rightarrow 0$, where $o(1)$ is uniformly on $E_{\delta}^{p}$. It remains to show that $\mid\left(x_{1}, y_{1}, s\right)-$ a $\mid / \bar{G}\left(x_{1}, y_{1}, s\right)$ is bounded on $E_{\delta}^{p}$ for small $\delta>0$. Assume without loss of generality that $\left|x_{1}\right| \leq\left|y_{1}\right|<a_{1}$, and in a first step consider the numerator of the right-most fraction of $(5.32)$. With Lemma 5.18 we obtain for $(x, y) \in E_{\delta}^{p}$

$$
\begin{aligned}
\left|\left(x_{1}, y_{1}, s\right)-\mathbf{a}\right| & =\sqrt{\left(a_{1}+x_{1}\right)^{2}+\left(a_{1}-y_{1}\right)^{2}+s^{2}} \\
& =\left(a_{1}+x_{1}\right) \sqrt{1+\left(\frac{a_{1}-y_{1}}{a_{1}+x_{1}}\right)^{2}+\left(\frac{s}{a_{1}+x_{1}}\right)^{2}} \\
& \leq\left(a_{1}+x_{1}\right) \sqrt{2+\left(\frac{p\left(2 a_{2}\right)^{p}}{a_{1}}\right)^{2}} .
\end{aligned}
$$

In a second step we look at the denominator and use $(5.31)$ to deduce that

$$
\begin{aligned}
& \bar{G}\left(x_{1}, y_{1}, s\right) \\
= & \left(a_{1}+x_{1}\right)+\left(a_{1}-y_{1}\right)-\frac{s}{p\left(2 a_{1}\right)^{p-1}} \\
\geq & \left(a_{1}+x_{1}\right)+\left(a_{1}-y_{1}\right)-\frac{1}{p\left(2 a_{1}\right)^{p-1}}\left(a_{1}+x_{1}\right)\left(\frac{a_{2}}{a_{1}}\right)^{p} \cdot p \cdot\left(2 a_{1}\right)^{p-1} \cdot\left(1+\frac{a_{1}-y_{1}}{a_{1}+x_{1}}\right) \\
= & \left(a_{1}+x_{1}\right) \cdot\left(1+\frac{a_{1}-y_{1}}{a_{1}+x_{1}}-\left(\frac{a_{2}}{a_{1}}\right)^{p} \cdot\left(1+\frac{a_{1}-y_{1}}{a_{1}+x_{1}}\right)\right) \\
= & \left(a_{1}+x_{1}\right) \cdot\left(1-\left(\frac{a_{2}}{a_{1}}\right)^{p}\right) \cdot\left(1+\frac{a_{1}-y_{1}}{a_{1}+x_{1}}\right) .
\end{aligned}
$$

From $\left|x_{1}\right| \leq\left|y_{1}\right|<a_{1}$ we have $0<a_{1}-y_{1} \leq a_{1}+x_{1}$ and hence $\frac{a_{1}-y_{1}}{a_{1}+x_{1}}>0$. Putting both parts together yields

$$
\frac{\left|\left(x_{1}, y_{1}, s\right)-\mathbf{a}\right|}{\bar{G}\left(x_{1}, y_{1}, s\right)} \leq \frac{\left(a_{1}+x_{1}\right) \cdot \sqrt{2+\left(\frac{p\left(2 a_{2}\right)^{p}}{a_{1}}\right)^{2}}}{\left(a_{1}+x_{1}\right) \cdot\left(1-\left(\frac{a_{2}}{a_{1}}\right)^{p}\right)}=\frac{\sqrt{2+\left(\frac{p\left(2 a_{2}\right)^{p}}{a_{1}}\right)^{2}}}{1-\left(\frac{a_{2}}{a_{1}}\right)^{p}}
$$

and the proof is finished. 


\subsubsection{The Shape of $E^{p}$ Close to The Poles}

As before we shift the set $E_{\ell}^{p}$ to the right by $a_{1} \cdot \mathbf{e}_{1}$ along the $z_{1}$-axis and call this set $P_{1}^{p}$. We get

$$
\begin{aligned}
P_{1}^{p} & =\left\{z \in \mathbb{R}^{d}: 0 \leq z_{1} \leq a_{1},\left(\frac{\left|z_{1}-a_{1}\right|}{a_{1}}\right)^{p}+\sum_{k=2}^{d}\left(\frac{\left|z_{k}\right|}{a_{k}}\right)^{p} \leq 1\right\} \\
& =\left\{z \in \mathbb{R}^{d}: 0 \leq z_{1} \leq a_{1},\left(1-\frac{z_{1}}{a_{1}}\right)^{p}+\sum_{k=2}^{d}\left(\frac{\left|z_{k}\right|}{a_{k}}\right)^{p} \leq 1\right\} .
\end{aligned}
$$

Arguing in nearly the same way as in Remark 4.9 and observing the Taylor series expansion of $|x-y|_{p}$ in $(5.29)$, we are led to the definitions

$$
\nu:=\frac{1}{d+p-1}
$$

and

$$
T_{n}(z):=\left(n^{p \nu} z_{1}, n^{\nu} \widetilde{z}\right), \quad z \in \mathbb{R}^{d}, n \in \mathbb{N} .
$$

Now we can state and prove an adapted version of Lemma 4.6:

Lemma 5.21. Suppose the random vector $V=\left(V_{1}, \ldots, V_{d}\right)$ has a density $g$ on $P_{1}^{p}$ with $g(z)=p(1+o(1))$ as $\delta \rightarrow 0$, uniformly on $P_{1}^{p} \cap\left\{z_{1} \leq \delta\right\}$ for some $p>0$. Then, for each bounded Borel set $B \subset \mathbb{R}^{d}$, we have $\mathbb{P}\left(T_{n}(V) \in B\right)=\kappa_{n}(B) / n$ with $\left.\kappa_{n}(B) \rightarrow p \cdot m_{d}\right|_{P^{p}}(B)$.

Proof. The proof closely parallels that of Lemma 4.6. Notice that the redefinition of $\nu$ makes sure that

$$
\Delta T_{n}(x)=\operatorname{det}\left(\operatorname{diag}\left(n^{p \nu}, n^{\nu}, \ldots, n^{\nu}\right)\right)=n^{(d+p-1) \nu}=n
$$

The only difference lies in the convergence of the indicator functions $\mathbb{1}\left\{z \in P_{n}^{p}\right\}$, where $P_{n}^{p}:=T_{n}\left(P_{1}^{p}\right)$. With $(1-t)^{p}=1-p t+O\left(t^{2}\right)$ for $t$ close to 0 we get

$$
\begin{aligned}
P_{n}^{p} & =\left\{z \in \mathbb{R}^{d}: 0 \leq \frac{z_{1}}{n^{p \nu}} \leq a_{1},\left(1-\frac{z_{1}}{a_{1} n^{p \nu}}\right)^{p}+\sum_{k=2}^{d}\left(\frac{\left|z_{k}\right|}{a_{k} n^{\nu}}\right)^{p} \leq 1\right\} \\
& =\left\{z \in \mathbb{R}^{d}: 0 \leq z_{1} \leq n^{p \nu} a_{1}, 1-p \frac{z_{1}}{a_{1} n^{p \nu}}+O\left(\frac{1}{n^{2 p \nu}}\right)+\sum_{k=2}^{d}\left(\frac{\left|z_{k}\right|}{a_{k} n^{\nu}}\right)^{p} \leq 1\right\} \\
& =\left\{z \in \mathbb{R}^{d}: 0 \leq z_{1} \leq n^{p \nu} a_{1}, O\left(\frac{1}{n^{p \nu}}\right)+\sum_{k=2}^{d}\left(\frac{\left|z_{k}\right|}{a_{k}}\right)^{p} \leq \frac{p z_{1}}{a_{1}}\right\}
\end{aligned}
$$


and hence $\mathbb{1}\left\{z \in P_{n}^{p}\right\} \rightarrow \mathbb{1}\left\{z \in P^{p}\right\}$ for almost all $z \in \mathbb{R}^{d}$, observe (5.28) for the definition of $P^{p}$.

As in Remark 4.7, we need to take a look at the state space of the point processes:

Remark 5.22. For each $p \in[1, \infty)$ we have $(1-t)^{p}-(1-p t) \geq 0$ for $t$ close enough to 0. In other words: The part $O\left(n^{-p \nu}\right)$ figuring in $(5.33)$ is always positive for large enough $n$. This fact yields $P_{n}^{p} \subset P^{p}$, at least for sufficiently large $n$. So, we can simply use $P^{p}$ as state space for the point processes in this setting.

With Lemma 5.21 we can copy Lemma 4.8 almost completely, we only have to replace $P\left(H_{i}\right)$ with $P^{p}$.

\subsubsection{MAIN PART OF THE PROOF OF THEOREM 5.15}

As stated before, the main part of the proof is very similar to that of Theorem 3.5 in Section 4.3. Hence, we will only elaborate on the (small) differences.

Proof. According to Lemma 5.20, for each $\varepsilon>0$ there is some $\delta>0$ so that

$$
\bar{G}\left(x_{1}, y_{1}, s\right)(1-\varepsilon) \leq 2 a-|x-y|_{p} \leq \bar{G}\left(x_{1}, y_{1}, s\right)(1+\varepsilon)
$$

for each $(x, y) \in E_{\delta}^{p}$. Now we define the random variables $X_{1}, X_{2}, \ldots, Y_{1}, Y_{1}, \ldots$ and $I_{n}$ in the same way as in the proof of Theorem 3.5 and put

$$
S_{i j}:=\left|\widetilde{X}_{i}-\widetilde{Y}_{j}\right|_{p}^{p}
$$

As seen in 4.13$)$, it suffices to examine $\min _{(i, j) \in I_{n}}\left\{n^{p \nu} \bar{G}\left(X_{i}, Y_{j}, S_{i j}\right)\right\}$. In view of (5.30) we get

$$
\begin{aligned}
n^{p \nu} \bar{G}\left(X_{i}, Y_{j}, S_{i j}\right) & =n^{p \nu}\left(a_{1}+X_{i, 1}\right)+n^{p \nu}\left(a_{1}-Y_{j, 1}\right)-\frac{1}{p\left(2 a_{1}\right)^{p-1}}\left|n^{\nu} \widetilde{X}_{i}-n^{\nu} \widetilde{Y}_{j}\right|_{p}^{p} \\
& =G\left(n^{p \nu}\left(a_{1}+X_{i, 1}\right), n^{\nu} \widetilde{X}_{i}, n^{p \nu}\left(a_{1}-Y_{j, 1}\right), n^{\nu} \widetilde{Y}_{j}\right)
\end{aligned}
$$

where

$$
G:\left\{\begin{array}{l}
P^{p} \times P^{p} \rightarrow \mathbb{R}_{+}, \\
(x, y) \mapsto x_{1}+y_{1}-\frac{1}{p\left(2 a_{1}\right)^{p-1}}|\widetilde{x}-\widetilde{y}|_{p}^{p} .
\end{array}\right.
$$

Based on this function $G$ we define the mapping $\widehat{G}$ as seen in (4.16). As in the proof of Theorem 3.5, we need that this function $\widehat{G}$ is continuous. In view of the proof of Lemma 4.10, we only have to show that $G(x, y) \geq c \cdot\left(x_{1}+y_{1}\right)$ for some $c>0$ and 
each $(x, y) \in P^{p} \times P^{p}$. The triangle inequality and the convexity of $t \mapsto t^{p}$ yield

$$
|\widetilde{x}-\widetilde{y}|_{p}^{p} \leq\left(|\widetilde{x}|_{p}+|\widetilde{y}|_{p}\right)^{p}=2^{p}\left(\frac{1}{2} \cdot|\widetilde{x}|_{p}+\frac{1}{2} \cdot|\widetilde{y}|_{p}\right)^{p} \leq 2^{p-1}\left(|\widetilde{x}|_{p}^{p}+|\widetilde{y}|_{p}^{p}\right),
$$

and since $z \in P^{p}$ implies $|\widetilde{z}|_{p}^{p} \leq \frac{p a_{2}^{p}}{a_{1}} \cdot z_{1}$, we get

$$
\begin{aligned}
G(x, y) & =x_{1}+y_{1}-\frac{1}{p\left(2 a_{1}\right)^{p-1}}|\widetilde{x}-\widetilde{y}|_{p}^{p} \\
& \geq x_{1}+y_{1}-\frac{1}{p\left(2 a_{1}\right)^{p-1}} \cdot 2^{p-1}\left(|\widetilde{x}|_{p}^{p}+|\widetilde{y}|_{p}^{p}\right) \\
& \geq x_{1}+y_{1}-\frac{1}{p a_{1}^{p-1}} \cdot\left(\frac{p a_{2}^{p}}{a_{1}} \cdot x_{1}+\frac{p a_{2}^{p}}{a_{1}} \cdot y_{1}\right) \\
& =x_{1}+y_{1}-\left(\frac{a_{2}}{a_{1}}\right)^{p} \cdot\left(x_{1}+y_{1}\right) \\
& =\left(1-\left(\frac{a_{2}}{a_{1}}\right)^{p}\right) \cdot\left(x_{1}+y_{1}\right) .
\end{aligned}
$$

From $a_{1}>a_{2}$ and the arguments given in the proof of Lemma 4.10, the continuity of $\widehat{G}$ follows. The remaining parts are clear.

\subsection{No SMOOTHNESS AT THE POLES}

In this section we illustrate that the smoothness of $E$ at the poles (Condition 2$)$ is not necessary in order to obtain limiting results similar to those stated throughout this work. As an easy example, we take $d \geq 3$ and consider the set

$$
\widehat{E}:=\bigcap_{i=2}^{d} E_{i}
$$

with

$$
E_{i}:=\left\{z \in \mathbb{R}^{d}:\left(\frac{z_{1}}{a_{1}}\right)^{2}+\left(\frac{z_{i}}{a_{i}}\right)^{2} \leq 1\right\}
$$

and $a_{1}>a_{2} \geq a_{3} \geq \ldots \geq a_{d}>0$, so that $a_{1}>\sqrt{a_{2}^{2}+\ldots+a_{d}^{2}}$. For an illustration of this set in three dimensions, see Figure 5.36 on page 113 . First of all, we have to demonstrate that $\widehat{E}$ has a unique diameter, so that Condition 1 is fulfilled. This property is an implication of the following lemma: 
Lemma 5.23. The set $\widehat{E}$ is a subset of a d-dimensional ellipsoid with half-axes $a_{1}, \sqrt{a_{2}^{2}+\ldots+a_{d}^{2}}, \ldots, \sqrt{a_{2}^{2}+\ldots+a_{d}^{2}}$.

Proof. For each $z \in \widehat{E}$ we have

$$
\begin{aligned}
& \left(\frac{z_{1}}{a_{1}}\right)^{2}+\left(\frac{z_{2}}{\sqrt{a_{2}^{2}+\ldots+a_{d}^{2}}}\right)^{2}+\ldots+\left(\frac{z_{d}}{\sqrt{a_{2}^{2}+\ldots+a_{d}^{2}}}\right)^{2} \\
= & \left(\frac{z_{1}}{a_{1}}\right)^{2}+\frac{z_{2}^{2}+\ldots+z_{d}^{2}}{a_{2}^{2}+\ldots+a_{d}^{2}} \\
= & \left(\frac{z_{1}}{a_{1}}\right)^{2} \sum_{i=2}^{d} \frac{a_{i}^{2}}{a_{2}^{2}+\ldots+a_{d}^{2}}+\sum_{i=2}^{d} \frac{a_{i}^{2}}{a_{2}^{2}+\ldots+a_{d}^{2}}\left(\frac{z_{i}}{a_{i}}\right)^{2} \\
= & \sum_{i=2}^{d} \frac{a_{i}^{2}}{a_{2}^{2}+\ldots+a_{d}^{2}}\left[\left(\frac{z_{1}}{a_{1}}\right)^{2}+\left(\frac{z_{i}}{a_{i}}\right)^{2}\right] \\
\leq & \sum_{i=2}^{d} \frac{a_{i}^{2}}{a_{2}^{2}+\ldots+a_{d}^{2}}=1 .
\end{aligned}
$$

This result and the choice $a_{1}>\sqrt{a_{2}^{2}+\ldots+a_{d}^{2}}$ make clear that the set $\widehat{E}$ has a unique diameter between the poles $\left(-a_{1}, \mathbf{0}\right)$ and $\left(a_{1}, \mathbf{0}\right)$. But the boundary of $\widehat{E}$ is not smooth at these points, i.e. Condition 2 is not fulfilled. Nevertheless, we can show a limiting result for densities supported by $\widehat{E}$, that are continuous and bounded away from 0 at the poles (as seen before in Condition 4). For this purpose, we define the new limiting set

$$
\widehat{P}:=\left\{z \in \mathbb{R}^{d}:\left(\frac{z_{2}}{a_{2}}\right)^{2} \leq \frac{2 z_{1}}{a_{1}}, \ldots,\left(\frac{z_{d}}{a_{d}}\right)^{2} \leq \frac{2 z_{1}}{a_{1}}\right\} .
$$

Figure 5.36 displays the sets $\widehat{E}$ (left) and $\widehat{P}$ (right) for the case $d=3, a_{1}=1$, $a_{2}=a_{3}=1 / 2$.

Theorem 5.24. Let $f$ fulfill Condition 4 for $a=a_{1}$ and with $E$ replaced with $\widehat{E}$. We then have

$$
n^{\frac{2}{d+1}}\left(2 a_{1}-\operatorname{diam}\left(\mathbf{Z}_{n}\right)\right) \stackrel{\mathcal{D}}{\longrightarrow} \min _{i, j \geq 1}\left\{\mathcal{X}_{i, 1}+\mathcal{Y}_{j, 1}-\frac{1}{4 a_{1}}\left|\widetilde{\mathcal{X}}_{i}-\widetilde{\mathcal{Y}}_{j}\right|^{2}\right\}
$$

where $\left\{\mathcal{X}_{i}, i \geq 1\right\} \stackrel{\mathcal{D}}{=} \operatorname{PRM}\left(\left.p_{\ell} \cdot m_{d}\right|_{\widehat{P}}\right)$ and $\left\{\mathcal{Y}_{j}, j \geq 1\right\} \stackrel{\mathcal{D}}{=} \operatorname{PRM}\left(\left.p_{r} \cdot m_{d}\right|_{\widehat{P}}\right)$ are independent Poisson processes. The same holds true if we replace $\operatorname{diam}\left(\mathbf{Z}_{n}\right)$ with $M_{n}$. 

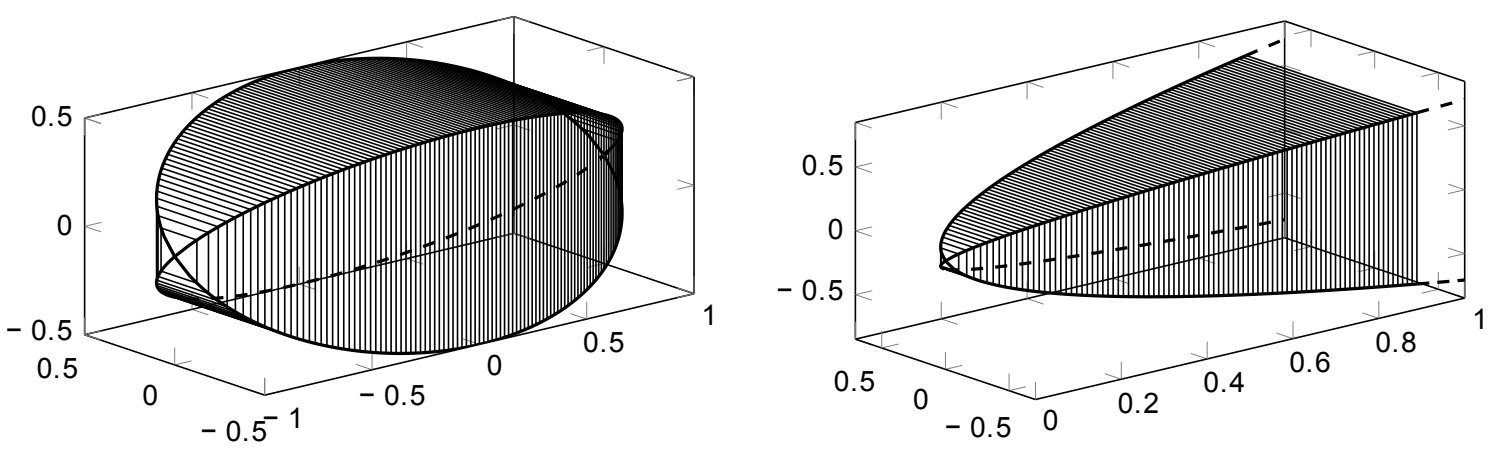

Figure 5.36: The boundaries of the sets $\widehat{E}$ (left) and $\widehat{P}$ (right) for the case $d=3$, $a_{1}=1, a_{2}=a_{3}=1 / 2$.

Proof. The only relevant change in comparison to the proof of Theorem 3.5 occurs in the proof of Lemma 4.6. Shifting the set $\widehat{E} \cap\left\{z_{1}<0\right\}$ to the right by $a_{1} \cdot \mathbf{e}_{1}$ along the $z_{1}$-axis and calling this set $\widehat{P}_{1}$ yields

$$
\widehat{P}_{1}=\bigcap_{i=2}^{d}\left\{z \in \mathbb{R}^{d}:\left(\frac{z_{1}-a_{1}}{a_{1}}\right)^{2}+\left(\frac{z_{i}}{a_{i}}\right)^{2} \leq 1,0 \leq z_{1}<a_{1}\right\} .
$$

Then, the corresponding part in the proof of Lemma 4.6 changes to

$$
\begin{aligned}
T_{n}\left(\widehat{P}_{1}\right) & =\left\{z \in \mathbb{R}^{d}: T_{n}^{-1}(z) \in \widehat{P}_{1}\right\} \\
& =\bigcap_{i=2}^{d}\left\{z \in \mathbb{R}^{d}:\left(\frac{\frac{z_{1}}{n^{2 \nu}}-a_{1}}{a_{1}}\right)^{2}+\left(\frac{z_{i}}{n^{\nu} a_{i}}\right)^{2} \leq 1,0 \leq \frac{z_{1}}{n^{2 \nu}}<a_{1}\right\} \\
& =\bigcap_{i=2}^{d}\left\{z \in \mathbb{R}^{d}: \frac{z_{1}^{2}}{n^{4 \nu} a_{1}^{2}}-\frac{2 z_{1}}{n^{2 \nu} a_{1}}+1+\left(\frac{z_{i}}{n^{\nu} a_{i}}\right)^{2} \leq 1,0 \leq \frac{z_{1}}{n^{2 \nu}}<a_{1}\right\} \\
& =\bigcap_{i=2}^{d}\left\{z \in \mathbb{R}^{d}: \frac{z_{1}^{2}}{n^{2 \nu} a_{1}^{2}}+\left(\frac{z_{i}}{a_{i}}\right)^{2} \leq \frac{2 z_{1}}{a_{1}}, 0 \leq z_{1}<a_{1} n^{2 \nu}\right\},
\end{aligned}
$$

and we obtain $\mathbb{1}\left\{z \in T_{n}\left(\widehat{P}_{1}\right)\right\} \rightarrow \mathbb{1}\{z \in \widehat{P}\}$ for almost all $z \in \mathbb{R}^{d}$.

We want to illustrate this theorem for the case that the underlying points are uniformly distributed in $\widehat{E}$ :

Example 5.25. If we assume that $Z$ is the uniform distribution in $\widehat{E}$, we can apply Theorem 5.24 with

$$
p_{\ell}=p_{r}=\frac{1}{m_{d}(\widehat{E})}=\left(2^{d-1} \cdot \frac{\Gamma\left(\frac{d+1}{2}\right) \sqrt{\pi}}{\Gamma\left(\frac{d}{2}+1\right)} \cdot \prod_{i=1}^{d} a_{i}\right)^{-1}>0 .
$$

For $d=3, a_{1}=1$ and $a_{2}=a_{3}=1 / 2$, the result of a simulation study is illustrated in 
Figure 5.37. Notice that the corresponding sets $\widehat{E}$ and $\widehat{P}$ were already illustrated in Figure 5.36.

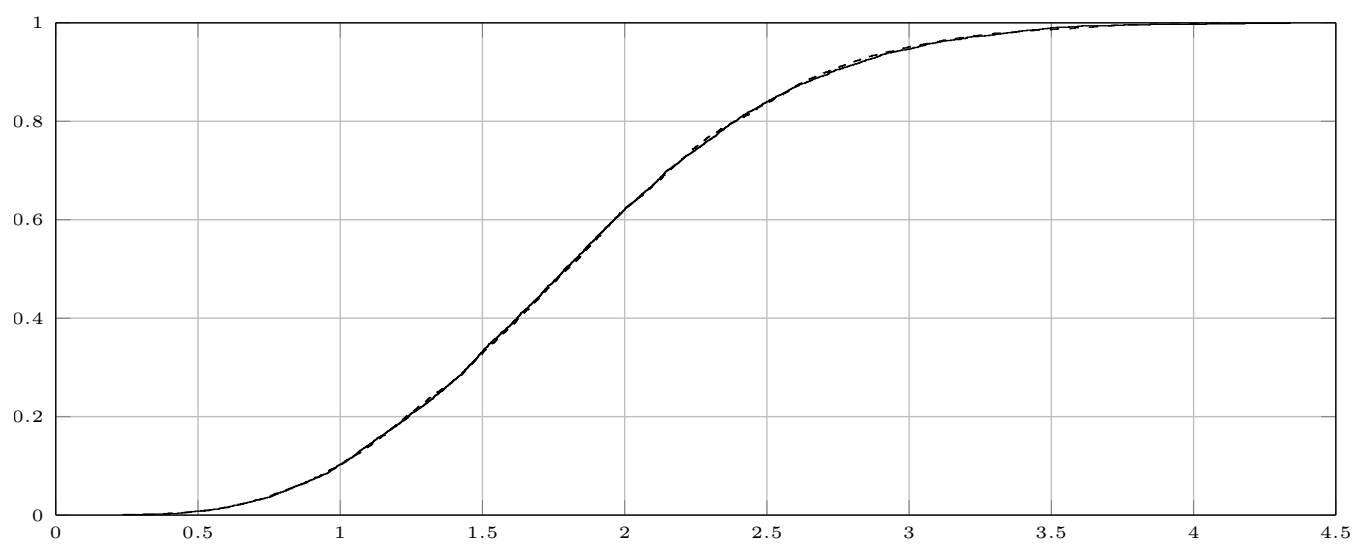

Figure 5.37: Empirical distribution function of $n^{1 / 2}\left(2-M_{n}\right)$ in the setting of Example 5.25 for $d=3$ with $a_{1}=1, a_{2}=a_{3}=1 / 2, n=1000$ (solid, 5000 replications). The limit distribution is approximated in the same way as described after Example 5.4 with $b \approx 3.65$ (dashed, 5000 replications).

For reasons of completeness, we show that the last equality figuring in (5.34) holds true. Writing

$$
\begin{aligned}
S\left(z_{1}\right): & =\left\{\widetilde{z} \in \mathbb{R}^{d-1}:\left(z_{1}, \widetilde{z}\right) \in \widehat{E}\right\} \\
& =\left\{\widetilde{z} \in \mathbb{R}^{d-1}:\left|z_{2}\right| \leq a_{2} \sqrt{1-\left(\frac{z_{1}}{a_{1}}\right)^{2}}, \ldots,\left|z_{d}\right| \leq a_{d} \sqrt{1-\left(\frac{z_{1}}{a_{1}}\right)^{2}}\right\}
\end{aligned}
$$

for $z_{1} \in\left[-a_{1}, a_{1}\right]$, we get

$$
m_{d-1}\left(S\left(z_{1}\right)\right)=\prod_{i=2}^{d} 2 a_{i} \sqrt{1-\left(\frac{z_{1}}{a_{1}}\right)^{2}}=2^{d-1}\left(1-\left(\frac{z_{1}}{a_{1}}\right)^{2}\right)^{\frac{d-1}{2}} \prod_{i=2}^{d} a_{i}
$$

and by applying Cavalieri's principle we obtain

$$
m_{d}(\widehat{E})=2 \int_{0}^{a_{1}} m_{d-1}\left(S\left(z_{1}\right)\right) \mathrm{d} z_{1}=2^{d}\left(\prod_{i=2}^{d} a_{i}\right) \int_{0}^{a_{1}}\left(1-\left(\frac{z_{1}}{a_{1}}\right)^{2}\right)^{\frac{d-1}{2}} \mathrm{~d} z_{1} .
$$


Substituting $\left(z_{1} / a_{1}\right)^{2}=s$ yields $z_{1}=a_{1} \sqrt{s}, \mathrm{~d} z_{1}=\frac{a_{1}}{2} s^{-\frac{1}{2}} \mathrm{~d} s$ and hence

$$
\begin{aligned}
m_{d}(\widehat{E}) & =2^{d}\left(\prod_{i=2}^{d} a_{i}\right) \cdot \frac{a_{1}}{2} \int_{0}^{1}(1-s)^{\frac{d-1}{2}} s^{-\frac{1}{2}} \mathrm{~d} s \\
& =2^{d-1}\left(\prod_{i=1}^{d} a_{i}\right) \cdot B\left(\frac{d+1}{2}, \frac{1}{2}\right) \\
& =2^{d-1} \cdot \frac{\Gamma\left(\frac{d+1}{2}\right) \sqrt{\pi}}{\Gamma\left(\frac{d}{2}+1\right)} \cdot \prod_{i=1}^{d} a_{i} .
\end{aligned}
$$





\section{CHAPTER 6}

\section{Generalizations 2 - Sets with no unique \\ DIAMETER}

In this section we consider sets with no unique diameter, i.e. we no longer assume that Condition 1 holds true. Basically, there are two different ways to modify this condition. The first is given by sets, having $k$ pairs of poles, where $1<k<\infty$, see Condition 10 below for a formal definition. Such sets will be studied in Subsection 6.1.1. An alternative modification of Condition 1 is - heuristically spoken in three dimensions given by sets with an equator, for example a three-dimensional ellipsoid with half-axes 1,1 and $1 / 2$. For Pearson Type II distributed points in $d$-dimensional ellipsoids with at least two but less than $d$ major half-axes, we still do not know whether a limit distribution for $M_{n}$ exists, or not. However, at least for each of these Pearson Type II distributions, Section 6.2 exhibits bounds for the limit distribution of $M_{n}$, provided that such a limit law exists.

\subsection{SEVERAL MAJOR AXES}

\subsubsection{General SETting}

In this section we consider closed sets with more than one, but finitely many pairs of poles. To this end, we formulate a more general version of Condition 1 : 
Condition 10. Let $E \subset \mathbb{R}^{d}$ be closed, $a>0, k \geq 2$ and $x^{(1)}, \ldots, x^{(k)}, y^{(1)}, \ldots, y^{(k)} \in$ $E$ so that

$$
\operatorname{diam}(E)=\left|x^{(1)}-y^{(1)}\right|=\ldots=\left|x^{(k)}-y^{(k)}\right|=2 a
$$

and

$$
\left(x^{(i)}, y^{(i)}\right) \neq\left(x^{(j)}, y^{(j)}\right) \neq\left(y^{(i)}, x^{(i)}\right)
$$

for $i \neq j$. Furthermore, we assume

$$
|x-y|<2 a \quad \text { for each } \quad(x, y) \in\left(E \backslash\left\{x^{(1)}, \ldots, x^{(k)}, y^{(1)}, \ldots, y^{(k)}\right\}\right) \times E .
$$

As in Condition 1, it is very important to assume $E$ to be closed, see the comments after Condition 1. Observe that (6.1) makes sure that no pair of poles (points with distance $2 a$ ) is considered twice. We want to emphasize the assumption $k<\infty$ in Condition 10. Sets with an equator - like an ellipsoid in $\mathbb{R}^{3}$ with half-axes $a_{1}=a_{2}>a_{3}-$ are explicitly excluded by this condition, see Section 6.2 for some considerations in this setting.

For $m \in\{1, \ldots, k\}$, let $\phi^{(m)}$ be a rigid motion of $\mathbb{R}^{d}$ with $\phi^{(m)}\left(x^{(m)}\right)=(-a, \mathbf{0})$ and $\phi^{(m)}\left(y^{(m)}\right)=(a, \mathbf{0})$. If $f$ is a density with support $E$, we write $f^{(m)}:=f \circ\left(\phi^{(m)}\right)^{-1}$ for the transformed density supported by $\phi^{(m)}(E)$. Our basic assumption in this section will be that, for each $m \in\{1, \ldots, k\}$, the set $\phi^{(m)}(E)$ and the density $f^{(m)}$ fulfill all the requirements of Theorem 3.5, formally:

Condition 11. For each $m \in\{1, \ldots, k\}$, we assume that $\phi^{(m)}(E)$ satisfies Conditions 2 and 3 , and that the density $f^{(m)}$ fulfills Condition 4 with respect to some constants $p_{\ell}^{(m)}, p_{r}^{(m)}>0$.

Figure 6.1 illustrates the setting under Conditions 10 and 11 in two dimensions for the case $k=2$. Appel et al. [3] investigated a similar setting in two dimensions for sets with boundary functions that - in contrast to Condition 11-decay faster to zero at the poles than a square-root. In that setting, it was necessary to demand that any two different major axes have no vertex in common. Under Condition 11, this requirement is given by definition: None of the points $x^{(1)}, \ldots, x^{(k)}, y^{(1)}, \ldots, y^{(k)}$ can be part of more than one pair of points with distance $2 a$, or, in other words, the set $E$ has exactly $2 k$ poles, see the following lemma.

Lemma 6.1. Under Conditions 10 and 11 we have

$$
\left|\left\{x^{(1)}, \ldots, x^{(k)}, y^{(1)}, \ldots, y^{(k)}\right\}\right|=2 k .
$$




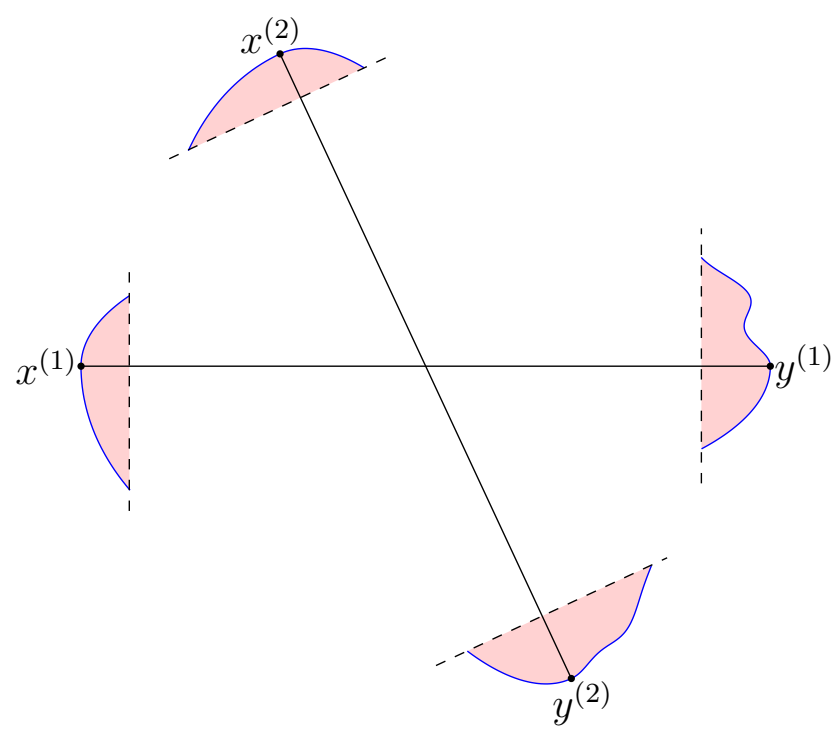

Figure 6.1: The setting under Condition 10 and Condition 11 in two dimensions for $k=2$.

Proof. Without loss of generality, we consider the case $x^{(1)}=(-a, \mathbf{0})$ and $y^{(1)}=(a, \mathbf{0})$, otherwise we move $E$ in a suitable way. Let $t \in\left\{x^{(2)}, \ldots, x^{(k)}, y^{(2)}, \ldots, y^{(k)}\right\}$ and assume that

$$
\left|x^{(1)}-t\right|=2 a,
$$

i.e. $t \in \partial B_{2 a}((-a, \mathbf{0}))$. In view of $(6.1)$, we obtain $t \neq y^{(1)}=(a, \mathbf{0})$ and together with $t \in \partial B_{2 a}((-a, \mathbf{0}))$, it follows that $\widetilde{t} \neq \mathbf{0}$, where $\widetilde{t}$ denotes the last $d-1$ components of $t$, as before. Hence, $-\mathbf{e}_{1}$ is no normal vector on the surface of the ball $B_{2 a}(t)$ at the point $x^{(1)}=(-a, \mathbf{0})$. Figure 6.2 illustrates this setting in the $\left(z_{1}, z_{2}\right)$-plane for the special case $t \in M_{r}$ and $t_{2} \neq 0$.

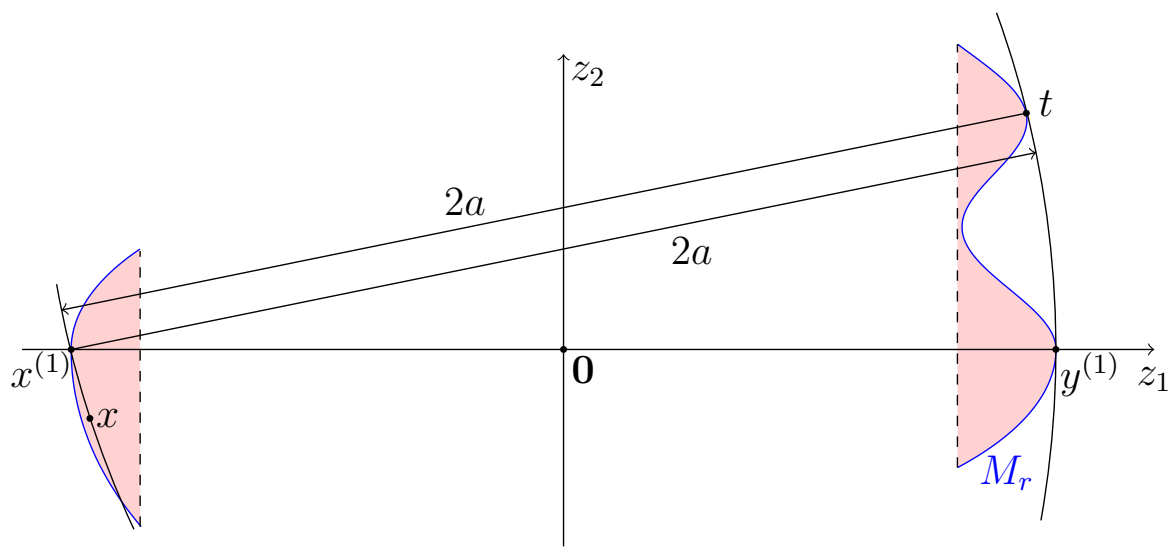

Figure 6.2: Illustration for the proof of Lemma 6.1. 
From Lemma 3.2 we know that the linear tangent space to $E$ at the left pole $x^{(1)}$ is the plane $\left\{z_{1}=0\right\}$. Putting these two parts together we can infer that there is a point $x$ in $\partial B_{2 a}(t)$ with $x \in \operatorname{int}(E)$. But this fact contradicts the standing assumption that $\operatorname{diam}(E)=2 a$.

Because of Lemma 6.1, there exists an $\varepsilon>0$ so that the balls

$$
B_{\varepsilon}\left(x^{(1)}\right), \ldots, B_{\varepsilon}\left(x^{(k)}\right), B_{\varepsilon}\left(y^{(1)}\right), \ldots, B_{\varepsilon}\left(y^{(k)}\right)
$$

are pairwise disjoint. For $m \in\{1, \ldots, k\}$ we define the set

$$
E^{(m)}:=E \cap\left(B_{\varepsilon}\left(x^{(m)}\right) \cup B_{\varepsilon}\left(y^{(m)}\right)\right)
$$

After moving $E^{(m)}$ via $\phi^{(m)}$ into the suitable position, Theorem 3.5 is applicable for each $m \in\{1, \ldots, k\}$. We consider again the Poisson process $\mathbf{Z}_{n}=\sum_{i=1}^{N_{n}} \varepsilon_{Z_{i}}$, defined in Section 2.2. Since the sets $E^{(1)}, \ldots, E^{(k)}$ are pairwise disjoint, the restrictions $\mathbf{Z}_{n}\left(\cdot \cap E^{(1)}\right), \ldots, \mathbf{Z}_{n}\left(\cdot \cap E^{(k)}\right)$ are independent Poisson processes. Consequently, for $m \in\{1, \ldots, k\}$, the maximum distances of points lying in $E^{(m)}$ are independent random variables. With

$$
I_{n}^{(m)}:=\left\{(i, j): 1 \leq i, j \leq N_{n},\left(Z_{i}, Z_{j}\right) \in E^{(m)} \times E^{(m)}\right\}
$$

for $m \in\{1, \ldots, k\}$, we obtain $I_{n}^{(m)} \neq \emptyset$ for sufficiently large $n$ for each $m \in\{1, \ldots, k\}$ almost surely and hence

$$
\begin{aligned}
2 a-\max _{1 \leq i, j \leq N_{n}}\left|Z_{i}-Z_{j}\right| & =2 a-\max _{1 \leq m \leq k}\left\{\max _{(i, j) \in I_{n}^{(m)}}\left|Z_{i}-Z_{j}\right|\right\} \\
& =2 a+\min _{1 \leq m \leq k}\left\{-\max _{(i, j) \in I_{n}^{(m)}}\left|Z_{i}-Z_{j}\right|\right\} \\
& =\min _{1 \leq m \leq k}\left\{2 a-\max _{(i, j) \in I_{n}^{(m)}}\left|Z_{i}-Z_{j}\right|\right\} .
\end{aligned}
$$

As mentioned before, we can apply Theorem 3.5 to each of the random variables $\max _{(i, j) \in I_{n}^{(m)}}\left|Z_{i}-Z_{j}\right|$, and since these $k$ random variables are independent for each $n \in \mathbb{N}$, the $k$ limiting random variables inherit this property. Hence, we obtain as limiting distribution of the maximum distance of points within $E$ a minimum of $k$ independent random variables, each of which can be described as seen in Theorem 3.5. After stating one last definition we can formulate a generalized version of our main 
result Theorem 3.5. Instead of $H_{\ell}$ and $H_{r}$ we write $H_{\ell}^{(m)}$ and $H_{r}^{(m)}$ for the Hessian matrices of the corresponding boundary functions of $E^{(m)}$ at the poles, $m \in\{1, \ldots, k\}$.

Theorem 6.2. Under Condition 10 and Condition 11 we have

$$
n^{\frac{2}{d+1}}\left(2 a-\operatorname{diam}\left(\mathbf{Z}_{n}\right)\right) \stackrel{\mathcal{D}}{\longrightarrow} \min _{1 \leq m \leq k} Z^{(m)}
$$

with independent random variables $Z^{(1)}, \ldots, Z^{(k)}$, fulfilling

$$
Z^{(m)} \stackrel{\mathcal{D}}{=} \min _{i, j \geq 1}\left\{\mathcal{X}_{i, 1}^{(m)}+\mathcal{Y}_{j, 1}^{(m)}-\frac{1}{4 a}\left|\widetilde{\mathcal{X}}_{i}^{(m)}-\widetilde{\mathcal{Y}}_{j}^{(m)}\right|^{2}\right\}
$$

where all the Poisson processes $\left\{\mathcal{X}_{i}^{(m)}, i \geq 1\right\} \stackrel{\mathcal{D}}{=} \operatorname{PRM}\left(\left.p_{\ell}^{(m)} \cdot m_{d}\right|_{P\left(H_{\ell}^{(m)}\right)}\right)$ and $\left\{\mathcal{Y}_{j}^{(m)}, j \geq 1\right\} \stackrel{\mathcal{D}}{=} \operatorname{PRM}\left(\left.p_{r}^{(m)} \cdot m_{d}\right|_{P\left(H_{r}^{(m)}\right)}\right), m \in\{1, \ldots, k\}$, are independent. The same result holds true if we replace $\operatorname{diam}\left(\mathbf{Z}_{n}\right)$ with $M_{n}$.

Example 6.3. For $d=2$ and $a_{1}>a_{2}$, consider the uniform distribution in a union of two ellipses

$$
E:=\left\{z \in \mathbb{R}^{2}:\left(\frac{z_{1}}{a_{1}}\right)^{2}+\left(\frac{z_{2}}{a_{2}}\right)^{2} \leq 1\right\} \cup\left\{z \in \mathbb{R}^{2}:\left(\frac{z_{1}}{a_{2}}\right)^{2}+\left(\frac{z_{2}}{a_{1}}\right)^{2} \leq 1\right\} .
$$

Then, we can apply Theorem 6.2 with $k=2$ and $a=a_{1}$. In this case, because of symmetry, the random variables $Z^{(1)}$ and $Z^{(2)}$ are not only independent, but also identically distributed. The calculation of the pertaining parameters is straightforward, cf. Remark 3.6. Several ways of generalizing this result are obvious: We can define such a union of more than two ellipsoids in higher dimensions with different minor half axes. Notice that it is not at all necessary that the major axes are orthogonal with respect to each other. Figure 6.3 shows one of these generalizations for $d=3$ with half-axes $1, \frac{1}{4}$ and $\frac{1}{4}$. 


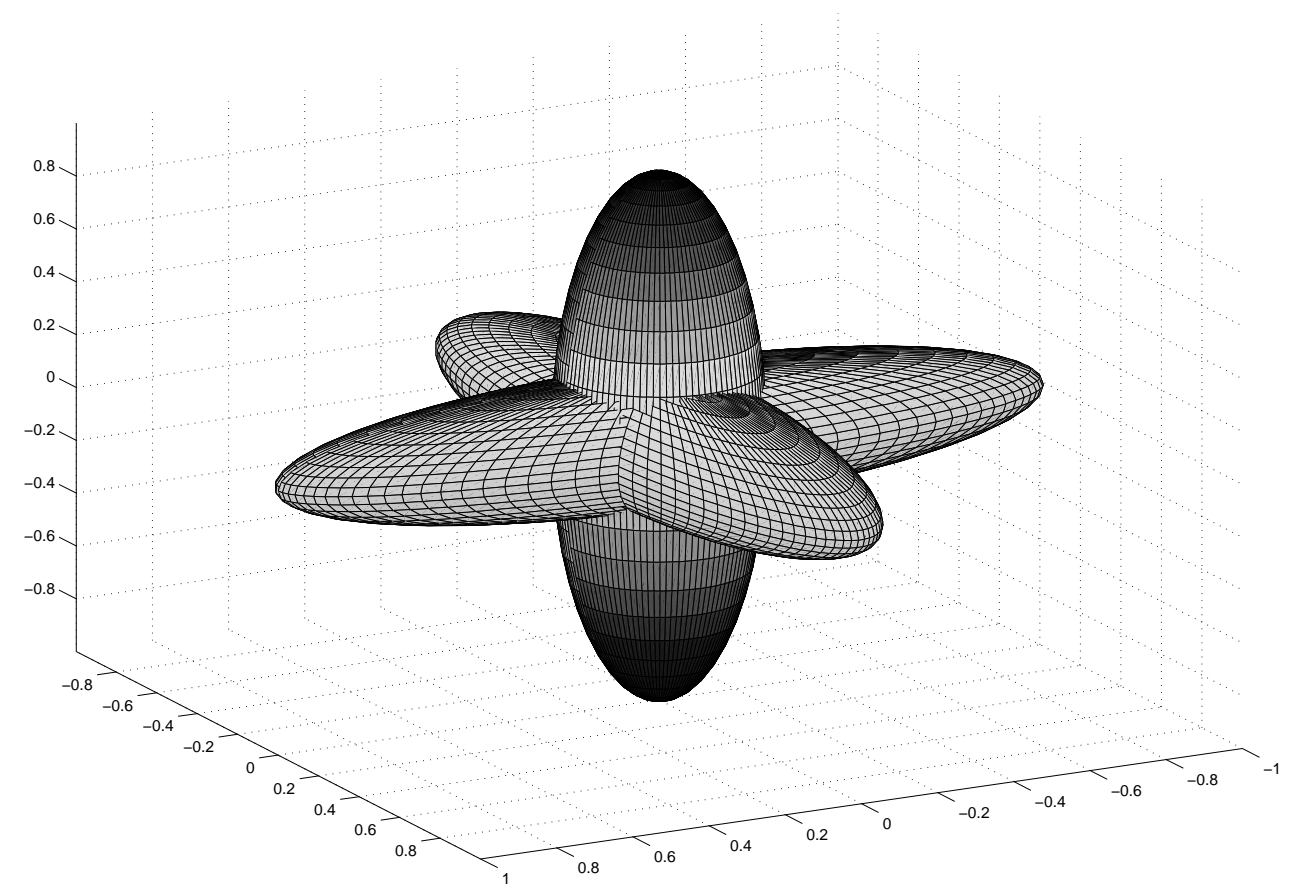

Figure 6.3: The union set of three ellipsoids in three dimensions with half-axes 1,1/4 and $1 / 4$.

\subsubsection{Application to $p$-BALls FOR $p>2$}

Consider for $p>2$ the ball of radius $r>0$ with respect to the $p$-norm, formally

$$
E_{r}^{p}:=\left\{z \in \mathbb{R}^{d}:|z|_{p} \leq r\right\}
$$

For $z \in \mathbb{R}^{d}$, Hölder's inequality gives

$$
\sum_{j=1}^{d} z_{j}^{2}=\sum_{j=1}^{d} z_{j}^{2} \cdot 1 \leq\left(\sum_{j=1}^{d}\left(z_{j}^{2}\right)^{\frac{p}{2}}\right)^{\frac{2}{p}} \cdot d^{1-\frac{2}{p}}=\left(\sum_{j=1}^{d}\left|z_{j}\right|^{p}\right)^{\frac{2}{p}} \cdot d^{1-\frac{2}{p}}=|z|_{p}^{2} \cdot d^{1-\frac{2}{p}}
$$

with equality only for

$$
\begin{aligned}
\left(z_{j}^{2}\right)^{\frac{p}{2}}=\frac{\sum_{k=1}^{d}\left(z_{k}^{2}\right)^{\frac{p}{2}}}{\sum_{k=1}^{d} 1} \cdot 1, \quad j \in\{1, \ldots, d\} & \Longleftrightarrow\left|z_{j}\right|^{p}=\frac{1}{d} \sum_{k=1}^{d}\left|z_{k}\right|^{p}, \quad j \in\{1, \ldots, d\} \\
& \Longleftrightarrow\left|z_{1}\right|=\ldots=\left|z_{d}\right| .
\end{aligned}
$$


Hence, for $z \in E_{r}^{p}$ we obtain $|z| \leq r \cdot d^{\frac{1}{2}-\frac{1}{p}}$, with equality only in the case

$$
\left|z_{1}\right|=\ldots=\left|z_{d}\right|=\left(\frac{r^{p}}{d}\right)^{\frac{1}{p}}=: \tau .
$$

The triangle inequality shows that the set $E_{r}^{p}$ has $2^{d-1}$ pairs of poles (i.e. $2^{d}$ poles), and each pole is given by $( \pm \tau, \ldots, \pm \tau)$. If $x^{(m)}$ is a pole, the corresponding opposite pole is $-x^{(m)}$, and we can conclude that

$$
\operatorname{diam}\left(E_{r}^{p}\right)=2 r d^{\frac{1}{2}-\frac{1}{p}}
$$

Hence, we put

$$
a:=\frac{\operatorname{diam}\left(E_{r}^{p}\right)}{2}=r d^{\frac{1}{2}-\frac{1}{p}} .
$$

The curvature of $\partial E_{r}^{p}$ at each of the $2^{d}$ poles is very easy to describe: At each pole, all $d-1$ principal curvatures coincide. Being more precise, we have the following result:

Lemma 6.4. At each of the $2^{d}$ poles, the boundary of $E_{r}^{p}$ has the principal curvature

$$
\kappa:=\frac{(p-1) d^{\frac{1}{p}-\frac{1}{2}}}{r}
$$

with multiplicity $d-1$.

The proof of this result will be given at the end of this subsection.

Remark 6.5. In the case $p=2$, which is deliberately excluded in this context, the calculations in the proof of Lemma 6.4 would be exactly the same, and so (6.3) would simplify to $\kappa=1 / r$ in each dimension. It is a well-known fact that $1 / r$ is the constant curvature of the boundary of an Euclidean ball with radius $r$. In Example 3.4 and Example 5.10 we have already used this result.

Using $p>2,(6.2)$ and $(6.3)$ we obtain

$$
\frac{1}{\kappa}+\frac{1}{\kappa}=\frac{2 r}{(p-1) d^{\frac{1}{p}-\frac{1}{2}}}=\frac{2 r d^{\frac{1}{2}-\frac{1}{p}}}{p-1}=\frac{2 a}{p-1}<2 a .
$$

Hence, condition (3.10), which in turn is sufficient for Condition 3, holds true. We thus can apply Theorem 6.2 with $k=2^{d-1}$ for suitable distributions supported by $E_{r}^{p}$, namely those with a density that is continuous and bounded away from 0 at each 
of the $2^{d}$ poles. The representation of the limiting sets stated in $(3.16)$ yields

$$
P\left(H_{\ell}^{(m)}\right)=P\left(H_{r}^{(m)}\right)=\left\{\left(z_{1}, \widetilde{z}\right) \in \mathbb{R}^{d}: \frac{\kappa}{2} \sum_{j=2}^{d} z_{j}^{2} \leq z_{1}\right\}
$$

for $m \in\left\{1, \ldots, 2^{d-1}\right\}$.

Example 6.6. The easiest example in this context is given by the uniform distribution in $E_{r}^{p}$. Using the aforesaid considerations and a formula for the volume of unit $p$-balls stated in Wang [25, we see that Theorem 6.2 is applicable putting $a=r d^{\frac{1}{2}-\frac{1}{p}}$, $k=2^{d-1}$ and

$$
p_{\ell}^{(1)}=p_{r}^{(1)}=\ldots=p_{\ell}^{\left(2^{d-1}\right)}=p_{r}^{\left(2^{d-1}\right)}=\frac{1}{m_{d}\left(E_{r}^{p}\right)}=\left(\frac{\left(2 r \Gamma\left(1+\frac{1}{p}\right)\right)^{d}}{\Gamma\left(1+\frac{d}{p}\right)}\right)^{-1}>0 .
$$

Figures 6.4 and 6.5 illustrate the results of a simulation study in this case for $d=2$ with $r=1, p \in\{3,10\}$ and $n=1000$.

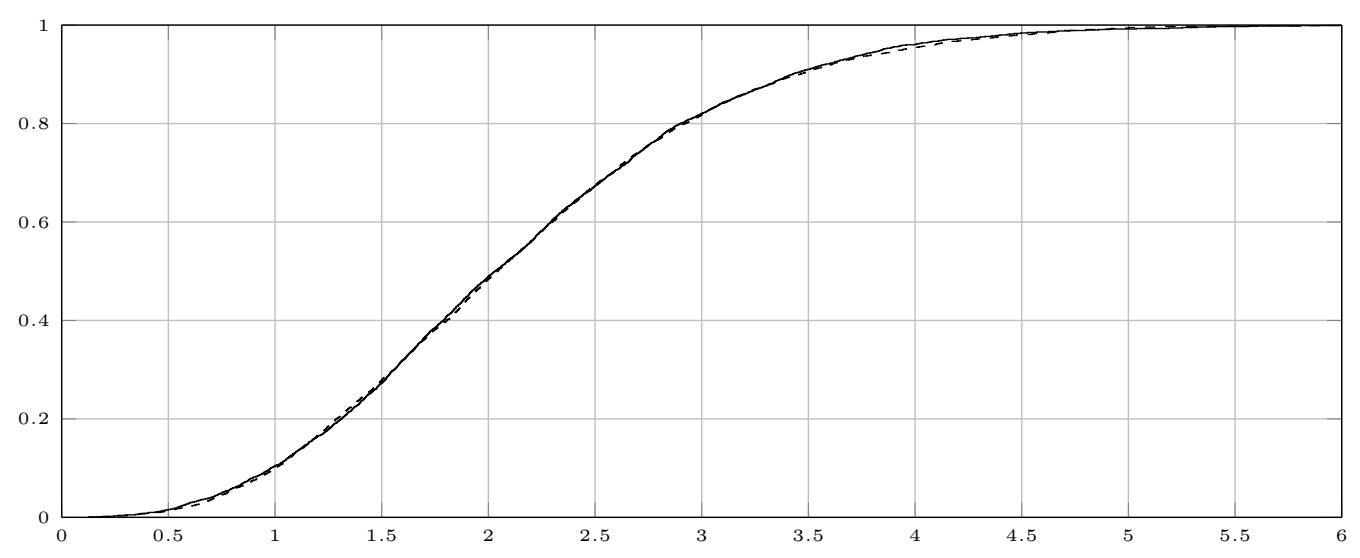

Figure 6.4: Empirical distribution function of $n^{2 / 3}\left(2 a-M_{n}\right)$ in the setting of Example 6.6 for $d=2$ with $r=1, p=3, n=1000$ (solid, 5000 replications). The limit distribution is approximated in the same way as described after Corollary 3.7 with $b=10$ (dashed, 5000 replications).

At least in principle, similar results can be obtained for general $p$-superellipsoids, given by

$$
\left\{z \in \mathbb{R}^{d}: \sum_{j=1}^{d}\left(\frac{\left|z_{j}\right|}{a_{j}}\right)^{p} \leq 1\right\}
$$

where $p>2$ and $a_{1} \geq a_{2} \geq \ldots \geq a_{d}$. But, without the assumption $a_{1}=\ldots=a_{d}$ of symmetry, the calculations in the proof of Lemma 6.4 can become very intricate. Moreover, without such an assumption, even the localisation of the poles can become 


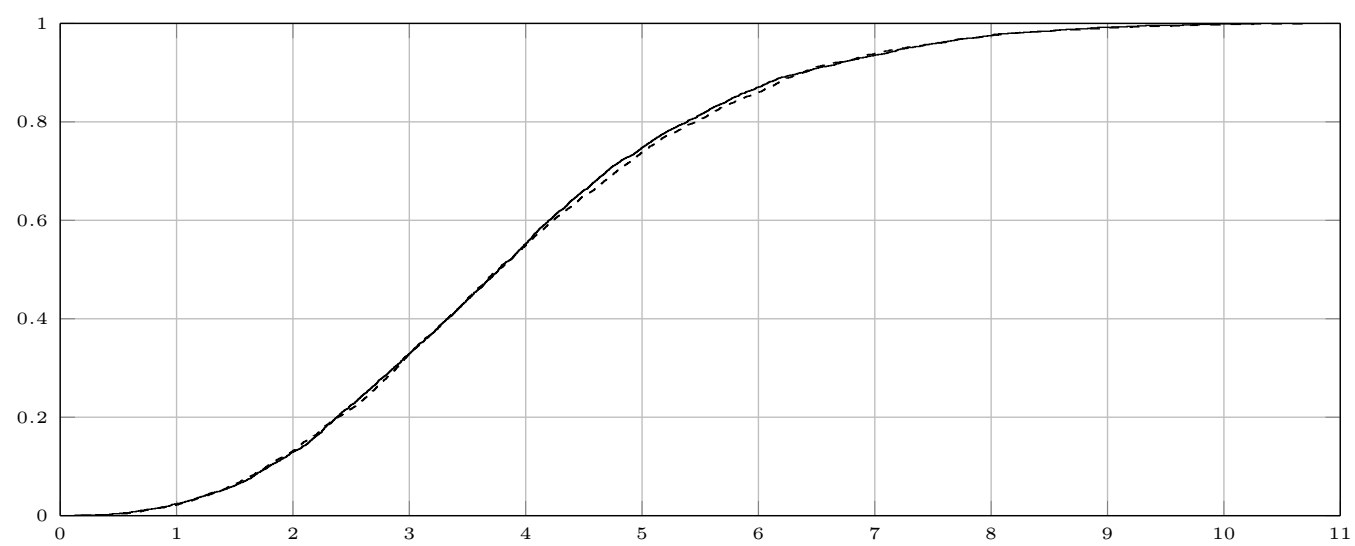

Figure 6.5: Empirical distribution function of $n^{2 / 3}\left(2 a-M_{n}\right)$ in the setting of Example 6.6 for $d=2$ with $r=1, p=10, n=1000$ (solid, 5000 replications). The limit distribution is approximated in the same way as described after Corollary 3.7 with $b=10$ (dashed, 5000 replications).

very complicated in higher dimensions. We omit details for this general setting and conclude this section with the missing proof of Lemma 6.4.

Proof of Lemma 6.4. From a purely formal perspective, we would have to rotate $E_{r}^{p}$ in such a way that one pair of poles is getting mapped to $(-a, \mathbf{0})$ and $(a, \mathbf{0})$, in order to be able to apply Theorem 3.5. But, since the principal curvatures are invariant under rigid motions, we can calculate them directly on the original set $E_{r}^{p}$. For reasons of symmetry, it suffices to investigate only the first 'hyper- $d$-tant' $\{z \geq 0\}:=\left\{z \in \mathbb{R}^{d}: z_{1} \geq 0, \ldots, z_{d} \geq 0\right\}$. For this set we have

$$
\partial E_{r}^{p} \cap\{z \geq 0\}=\left\{z \geq 0: z_{1}^{p}+\ldots+z_{d}^{p}=r^{p}\right\}
$$

and hence

$$
z_{1}=\left(r^{p}-z_{2}^{p}-\ldots-z_{d}^{p}\right)^{\frac{1}{p}}=: s(\widetilde{z}) .
$$

Defining $\mathbf{s}:\left\{\widetilde{z} \in \mathbb{R}_{+}^{d-1}: z_{2}^{p}+\ldots+z_{d}^{p}<r^{p}\right\} \rightarrow \mathbb{R}_{+}^{d}$ by $\mathbf{s}(\widetilde{z}):=(s(\widetilde{z}), \widetilde{z})$, we can use the results of Subsection A.2.2 to calculate the principal curvatures and directions of $\mathbf{s}$ at the point $\mathbf{s}\left(\widetilde{\tau}^{*}\right)$, where $\widetilde{\tau}^{*}=(\tau, \ldots, \tau) \in \mathbb{R}^{d-1}$. For $i, j \in\{2, \ldots, d\}$ we get

$$
s_{i}(\widetilde{z})=\frac{1}{p}\left(r^{p}-z_{2}^{p}-\ldots-z_{d}^{p}\right)^{\frac{1}{p}-1} \cdot\left(-p z_{i}^{p-1}\right)=-\left(r^{p}-z_{2}^{p}-\ldots-z_{d}^{p}\right)^{\frac{1}{p}-1} \cdot z_{i}^{p-1}
$$


and

$$
s_{i j}(\widetilde{z})= \begin{cases}-\left(\left(r^{p}-\sum_{k=2}^{d} z_{k}^{p}\right)^{\frac{1}{p}-1} z_{i}^{p-2}+\left(r^{p}-\sum_{k=2}^{d} z_{k}^{p}\right)^{\frac{1}{p}-2} z_{i}^{2 p-2}\right)(p-1) & , i=j \\ -\left(r^{p}-\sum_{k=2}^{d} z_{k}^{p}\right)^{\frac{1}{p}-2} z_{i}^{p-1} z_{j}^{p-1}(p-1) & , i \neq j\end{cases}
$$

With the matrices $\mathcal{G}\left(\widetilde{\tau}^{*}\right)$ and $\mathcal{B}\left(\widetilde{\tau}^{*}\right)$ as stated in Subsection A.2.2, we have to calculate the eigenvalues of the matrix

$$
\mathcal{L}\left(\widetilde{\tau}^{*}\right)=\mathcal{G}\left(\widetilde{\tau}^{*}\right)^{-1} \mathcal{B}\left(\widetilde{\tau}^{*}\right)
$$

Because of

$$
\begin{aligned}
s_{i}\left(\widetilde{\tau}^{*}\right) & =-\left(r^{p}-(d-1) \frac{r^{p}}{d}\right)^{\frac{1}{p}-1}\left(\frac{r^{p}}{d}\right)^{\frac{p-1}{p}} \\
& =-r^{1-p}\left(\frac{1}{d}\right)^{\frac{1-p}{p}}\left(\frac{r^{p}}{d}\right)^{\frac{p-1}{p}} \\
& =-r^{1-p+p-1}\left(\frac{1}{d}\right)^{\frac{1-p+p-1}{p}} \\
& =-1
\end{aligned}
$$

it follows from (A.1) that

$$
\mathcal{G}\left(\widetilde{\tau}^{*}\right)=\left(\begin{array}{ccccc}
2 & 1 & 1 & \ldots & 1 \\
1 & 2 & 1 & \ldots & 1 \\
\vdots & \ddots & \ddots & \ddots & \vdots \\
1 & \ldots & 1 & 2 & 1 \\
1 & \ldots & 1 & 1 & 2
\end{array}\right)
$$


For $i \neq j$ we have

$$
\begin{aligned}
s_{i j}\left(\widetilde{\tau}^{*}\right) & =-\left(r^{p}-(d-1) \frac{r^{p}}{d}\right)^{\frac{1}{p}-2}\left(\frac{r^{p}}{d}\right)^{\frac{p-1}{p}}\left(\frac{r^{p}}{d}\right)^{\frac{p-1}{p}}(p-1) \\
& =-r^{1-2 p}\left(\frac{1}{d}\right)^{\frac{1}{p}-2} r^{2 p-2}\left(\frac{1}{d}\right)^{2-\frac{2}{p}}(p-1) \\
& =-r^{-1}\left(\frac{1}{d}\right)^{-\frac{1}{p}}(p-1) \\
& =-\frac{(p-1) d^{\frac{1}{p}}}{r}
\end{aligned}
$$

and in the same way we obtain

$$
\begin{aligned}
& s_{i i}\left(\widetilde{\tau}^{*}\right) \\
= & -\left(\left(r^{p}-(d-1) \frac{r^{p}}{d}\right)^{\frac{1}{p}-1}\left(\frac{r^{p}}{d}\right)^{\frac{p-2}{p}}+\left(r^{p}-(d-1) \frac{r^{p}}{d}\right)^{\frac{1}{p}-2}\left(\frac{r^{p}}{d}\right)^{\frac{2 p-2}{p}}\right)(p-1) \\
= & -\left(r^{1-p}\left(\frac{1}{d}\right)^{\frac{1}{p}-1} r^{p-2}\left(\frac{1}{d}\right)^{1-\frac{2}{p}}+r^{1-2 p}\left(\frac{1}{d}\right)^{\frac{1}{p}-2} r^{2 p-2}\left(\frac{1}{d}\right)^{2-\frac{2}{p}}\right)(p-1) \\
= & -\left(r^{-1}\left(\frac{1}{d}\right)^{-\frac{1}{p}}+r^{-1}\left(\frac{1}{d}\right)^{-\frac{1}{p}}\right)(p-1) \\
= & -\frac{2(p-1) d^{\frac{1}{p}}}{r} .
\end{aligned}
$$

Now,

$$
\frac{1}{\sqrt{1+\sum_{j=2}^{d} s_{j}\left(\widetilde{\tau}^{*}\right)^{2}}}=\frac{1}{\sqrt{1+\sum_{j=2}^{d}(-1)^{2}}}=\frac{1}{\sqrt{1+d-1}}=\frac{1}{\sqrt{d}},
$$

and (A.2) yields

$$
\mathcal{B}\left(\widetilde{\tau}^{*}\right)= \pm \frac{1}{\sqrt{d}}\left(-\frac{(p-1) d^{\frac{1}{p}}}{r}\right)\left(\begin{array}{ccccc}
2 & 1 & 1 & \ldots & 1 \\
1 & 2 & 1 & \ldots & 1 \\
\vdots & \ddots & \ddots & \ddots & \vdots \\
1 & \ldots & 1 & 2 & 1 \\
1 & \ldots & 1 & 1 & 2
\end{array}\right)=\mp \frac{(p-1) d^{\frac{1}{p}-\frac{1}{2}}}{r} \mathcal{G}\left(\widetilde{\tau}^{*}\right) .
$$

We thus have

$$
\mathcal{L}\left(\widetilde{\tau}^{*}\right)=\mathcal{G}\left(\widetilde{\tau}^{*}\right)^{-1} \mathcal{B}\left(\widetilde{\tau}^{*}\right)=\mp \frac{(p-1) d^{\frac{1}{p}-\frac{1}{2}}}{r} \mathcal{G}\left(\widetilde{\tau}^{*}\right)^{-1} \mathcal{G}\left(\widetilde{\tau}^{*}\right)=\mp \frac{(p-1) d^{\frac{1}{p}-\frac{1}{2}}}{r} \mathrm{I}_{d-1} .
$$


We choose the inner unit normal vector in order to render the eigenvalues of $\mathcal{L}\left(\widetilde{\tau}^{*}\right)$ positive. Then, $\mathcal{L}\left(\widetilde{\tau}^{*}\right)$ possesses the eigenvalue

$$
\kappa=\frac{(p-1) d^{\frac{1}{p}-\frac{1}{2}}}{r}
$$

with multiplicity $d-1$. Since the eigenvalue $\kappa$ has multiplicity $d-1$, each direction in the tangent space of $\mathbf{s}$ at the pole $\mathbf{s}\left(\widetilde{\tau}^{*}\right)$ is a principal direction with respect to $\kappa$. Notice that all these calculations continue to hold if we put $p=2$ to show Remark 6.5.

\subsection{Ellipsoids WITH NO UNIQUE MAJOR HALF-AXIS}

\subsubsection{MAIN RESULtS}

In this section, we fix $d \geq 3$ and $e \in\{2, \ldots, d-1\}$ and consider the $d$-dimensional ellipsoid $E$ with half-axes $a_{1}=\ldots=a_{e}=1$ and $1>a_{e+1} \geq \ldots \geq a_{d}$, formally:

$$
E=\left\{z \in \mathbb{R}^{d}: z_{1}^{2}+\ldots+z_{e}^{2}+\left(\frac{z_{e+1}}{a_{e+1}}\right)^{2}+\ldots+\left(\frac{z_{d}}{a_{d}}\right)^{2} \leq 1\right\} .
$$

There is no loss of generality in assuming that the $e$ major half-axes have length 1 . Otherwise, one would only have to scale $E$ and $M_{n}$ in a suitable way. We assume that the points $Z_{1}, Z_{2}, \ldots$ are independent and identically distributed according to a Pearson Type II distribution with parameter $\beta>-1$ on $\operatorname{int}(E)$. This means that the density of $Z_{1}$ is given by

$$
f(z)=c_{1} \cdot\left(1-z^{\top} \Sigma^{-1} z\right)^{\beta} \cdot \mathbb{1}\{z \in \operatorname{int}(E)\}
$$

where $\Sigma:=\operatorname{diag}\left(1, \ldots, 1, a_{e+1}^{2}, \ldots, a_{d}^{2}\right) \in \mathbb{R}^{d \times d}$ and

$$
c_{1}:=\frac{\Gamma\left(\frac{d}{2}+\beta+1\right)}{\Gamma(\beta+1) \pi^{\frac{d}{2}} \prod_{i=e+1}^{d} a_{i}},
$$

see Example 5.4 and recall $a_{1}=\ldots=a_{e}=1$. Notice that we could use $E$ itself instead of $\operatorname{int}(E)$ as support of $f$ for $\beta \geq 0$. But, since $\partial E$ has no influence at all on the limiting behavior of $M_{n}$ in our setting, the consideration of $\operatorname{int}(E)$ instead of $E$ means no loss of generality. In this setting, we cannot state an exact limit theorem for

$$
M_{n}=\max _{1 \leq i, j \leq n}\left|Z_{i}-Z_{j}\right|
$$


However, by considering the projections $\bar{Z}_{1}, \bar{Z}_{2}, \ldots$ of $Z_{1}, Z_{2}, \ldots$ onto the first $e$ components and investigating

$$
\bar{M}_{n}:=\max _{1 \leq i, j \leq n}\left|\bar{Z}_{i}-\bar{Z}_{j}\right|
$$

we can establish bounds for the unknown limit distribution, if it exists. To this end, we consider $\mathbb{R}^{d}$ as $\mathbb{R}^{e} \times \mathbb{R}^{d-e}$ and write $\bar{z}:=\left(z_{1}, \ldots, z_{e}\right)$ for $z=\left(z_{1}, \ldots, z_{d}\right) \in \mathbb{R}^{d}$. In the same way, we put $\bar{Z}_{n}:=\left(Z_{n, 1}, \ldots, Z_{n, e}\right)$ for $Z_{n}=\left(Z_{n, 1}, \ldots, Z_{n, d}\right)$ and $n \in \mathbb{N}$. Obviously, the random variables $\bar{Z}_{1}, \bar{Z}_{2}, \ldots$ are independent and identically distributed. Taking some orthogonal matrix $Q_{e} \in \mathbb{R}^{e x e}$ and putting $Q:=\operatorname{diag}\left(Q_{e}, \mathrm{I}_{d-e}\right)$, the special form of $\Sigma$ yields

$$
f(Q z)=c_{1} \cdot\left(1-z^{\top} Q^{\top} \Sigma^{-1} Q z\right)^{\beta}=c_{1} \cdot\left(1-z^{\top} \Sigma^{-1} z\right)^{\beta}=f(z)
$$

for each $z \in \operatorname{int}(E)$, and we can conclude that the distribution of $\bar{Z}_{1}, \bar{Z}_{2}, \ldots$ is spherically symmetric on the unit ball $\mathbb{B}^{e}$. In addition to that, the proof of Lemma 6.8 will reveal that this distribution solely depends on $d, e$ and $\beta$, not on $a_{e+1}, \ldots, a_{d}$.

The great advantage of assuming $a_{1}=\ldots=a_{e}=1$ is that we can directly apply a result of Lao [16] for the maximum distance of the random points $\bar{Z}_{1}, \bar{Z}_{2}, \ldots$ lying in $\mathbb{B}^{e}$. Being more precise, we will use the following result:

Lemma 6.7 (Corollary 3.7 in Lao [16]). If the i.i.d. points $X_{1}, X_{2}, \ldots$ have a spherically symmetric distribution in $\mathbb{B}^{e}, e \geq 2$, and

$$
\mathbb{P}\left(1-\left|X_{1}\right| \leq s\right) \sim a s^{\alpha}
$$

as $s \downarrow 0$, we have

$$
\lim _{n \rightarrow \infty} \mathbb{P}\left(\left(\frac{\sigma}{2}\right)^{\frac{2}{e-1+4 \alpha}} \cdot n^{\frac{4}{e-1+4 \alpha}} \cdot\left(2-\max _{1 \leq i, j \leq n}\left|X_{i}-X_{j}\right|\right) \leq t\right)=1-\exp \left(-t^{\frac{e-1}{2}+2 \alpha}\right)
$$

$t>0$, where

$$
\sigma:=\frac{2^{e-2} \Gamma\left(\frac{e}{2}\right) a^{2} \Gamma(\alpha+1)^{2}}{\sqrt{\pi} \Gamma\left(\frac{e+1}{2}+2 \alpha\right)} .
$$

The next lemma shows that this result is applicable for the random variables $\bar{Z}_{1}, \bar{Z}_{2}, \ldots$ : 
Lemma 6.8. With

$$
a:=\frac{\Gamma\left(\frac{d}{2}+\beta+1\right)}{\Gamma\left(\frac{d-e}{2}+\beta+2\right)} \cdot \pi^{-\frac{e}{2}} \cdot e \cdot \omega_{e} \cdot 2^{\frac{d-e}{2}+\beta}
$$

and

$$
\alpha:=\frac{d-e}{2}+\beta+1
$$

we have

$$
\mathbb{P}\left(1-\left|\bar{Z}_{1}\right| \leq s\right) \sim a s^{\alpha}
$$

as $s \downarrow 0$

The proof of this lemma can be found in Subsection 6.2.2. Using the definition of $\sigma$ in Lemma 6.7 with $a$ and $\alpha$ given by Lemma 6.8, we put

$$
b_{n}:=\left(\frac{\sigma}{2}\right)^{\frac{2}{2 d-e+4 \beta+3}} \cdot n^{\frac{4}{2 d-e+4 \beta+3}}, \quad n \geq 1 .
$$

Furthermore, we let

$$
G(t):=1-\exp \left(-t^{\frac{2 d-e+4 \beta+3}{2}}\right)
$$

for $t \geq 0$. Regarding these definitions, notice that

$$
\begin{aligned}
\frac{2}{e-1+4 \alpha} & =\frac{2}{e-1+4\left(\frac{d-e}{2}+\beta+1\right)} \\
& =\frac{2}{e-1+2 d-2 e+4 \beta+4} \\
& =\frac{2}{2 d-e+4 \beta+3}
\end{aligned}
$$

and hence

$$
\frac{e-1}{2}+2 \alpha=\frac{e-1+4 \alpha}{2}=\frac{2 d-e+4 \beta+3}{2} .
$$

With Lemma 6.7 and Lemma 6.8 we get

$$
\mathbb{P}\left(b_{n}\left(2-\bar{M}_{n}\right) \leq t\right) \rightarrow G(t) .
$$

But, since our focus lies on the asymptotic behavior of of $\max _{1 \leq i, j \leq n}\left|Z_{i}-Z_{j}\right|$, not on that of $\max _{1 \leq i, j \leq n}\left|\bar{Z}_{i}-\bar{Z}_{j}\right|$, we have to find some useful relation between 
these two random variables. The key to success will be the following lemma, which provides bounds for $|x-y|, x, y \in E$, that depend merely on $\bar{x}, \bar{y}$ and the half-axis $a_{e+1}$.

Lemma 6.9. Putting

$$
g(\bar{x}, \bar{y}):=\sqrt{(|\bar{x}|+|\bar{y}|)^{2}+2 a_{e+1}^{2}\left(2-|\bar{x}|^{2}-|\bar{y}|^{2}\right)},
$$

we have

$$
|\bar{x}-\bar{y}| \leq|x-y| \leq g(\bar{x}, \bar{y})
$$

for all $x, y \in E$.

The proof of this lemma will be given in Subsection 6.2.2. Using the convergence given in (6.6) and Lemma 6.9, we can now state the main result of this section:

Theorem 6.10. Under the standing assumptions of this section we have

$$
\begin{aligned}
G(t) & \leq \liminf _{n \rightarrow \infty} \mathbb{P}\left(b_{n}\left(2-M_{n}\right) \leq t\right) \\
& \leq \limsup _{n \rightarrow \infty} \mathbb{P}\left(b_{n}\left(2-M_{n}\right) \leq t\right) \\
& \leq G\left(\frac{t}{1-a_{e+1}^{2}}\right), \quad t \geq 0,
\end{aligned}
$$

where $b_{n}$ and $G$ are given in (6.4) and (6.5), respectively.

Before we give the proof of Theorem 6.10, we want to state an important corollary and illustrate the result by means of a simulation study.

Corollary 6.11. From Theorem 6.10 we immediately know that the sequence

$$
\left(n^{\frac{4}{2 d-e+4 \beta+3}}\left(2-M_{n}\right)\right)_{n \in \mathbb{N}}
$$

is tight. So, if there are a positive sequence $\left(a_{n}\right)_{n \in \mathbb{N}}$ and a non-degenerate distribution function $F$ with $\mathbb{P}\left(a_{n}\left(2-M_{n}\right) \leq t\right) \rightarrow F(t), t \geq 0$, we can conclude that $a_{n} \sim$ $c \cdot n^{\frac{4}{2 d-e+4 \beta+3}}$ for some fixed $c \in \mathbb{R}$.

For our simulation study we only consider the uniform distribution in $E$, i.e. we put $\beta=0$. In a first step we take $d=3$ with $e=2$ for the four cases $a_{3} \in\{0.25,0.5,0.75,0.9\}$ and in a second step $d=6$ with $e=2, a_{3}=0.9$ and the two cases $a_{4}=\ldots=a_{6} \in\{0.1,0.9\}$. In each of the following figures, the empirical distribution function of $b_{n}\left(2-M_{n}\right)$ is plotted solid and that of $b_{n}\left(2-\bar{M}_{n}\right)$ dashed. In 
each case, the lower dotted curve is the graph of the function $G(t)$, the upper dotted curve that of $G\left(t /\left(1-a_{3}^{2}\right)\right)$. Since $M_{n} \geq \bar{M}_{n}$, the solid curve always lies above the dashed curve and from Lemma 6.7 and Lemma 6.8 we know that the dashed curve converges to the lower dotted curve as $n$ tends to infinity. For our simulation study we have chosen $n=100000$. In each of the following figures the convergence of the dashed curve to the limit law $G(t)$ is seen to be slow. For 'small' $a_{3}$, the difference between $M_{n}$ and $\bar{M}_{n}$ is 'small', too, and thus the dashed and the solid empirical distribution functions are lying close to each other, see for example Figure 6.6. In this case, the dotted bounding functions are lying close to each other, too. So, in this case Theorem 6.10 provides a small range for the possible limiting distribution of $b_{n}\left(2-M_{n}\right)$, but the inequalities given in (6.7) only hold for 'large' $n$. The larger we choose $a_{3}<1$, the bigger the difference between $M_{n}$ and $\bar{M}_{n}$ gets. Due to this fact, in this case the inequalities given in (6.7) can hold for smaller $n$, but the difference between the two dotted bounding functions can become very big, see Figures 6.9 to 6.11. Another interesting effect in higher dimensions can be observed by comparing Figures 6.10 and 6.11. The higher the number of half-axes of size $a_{e+1}$ gets, the bigger the difference between $M_{n}$ and $\bar{M}_{n}$ becomes. Due to this fact, the solid curve in Figure 6.11 $\left(d=6, a_{3}=\ldots=a_{6}=0.9\right)$ lies much more in the middle of the two dotted bounding functions than in Figure $6.10\left(d=6, a_{3}=0.9, a_{4}=a_{5}=a_{6}=0.1\right)$.

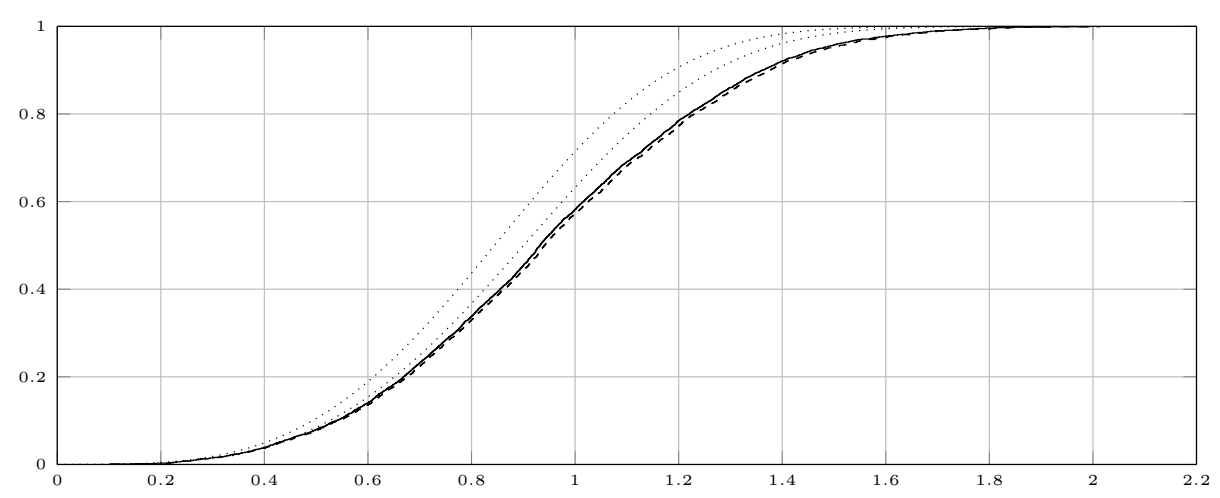

Figure 6.6: Empirical distribution functions of $b_{n}\left(2-M_{n}\right)$ (solid) and $b_{n}\left(2-\bar{M}_{n}\right)$ (dashed) for $d=3$ with $e=2, a_{3}=0.25$ and $n=100000$ (5000 replications). The dotted curves are the bounding functions $G(t)$ (lower) and $G\left(t /\left(1-0.25^{2}\right)\right)$ (upper). 


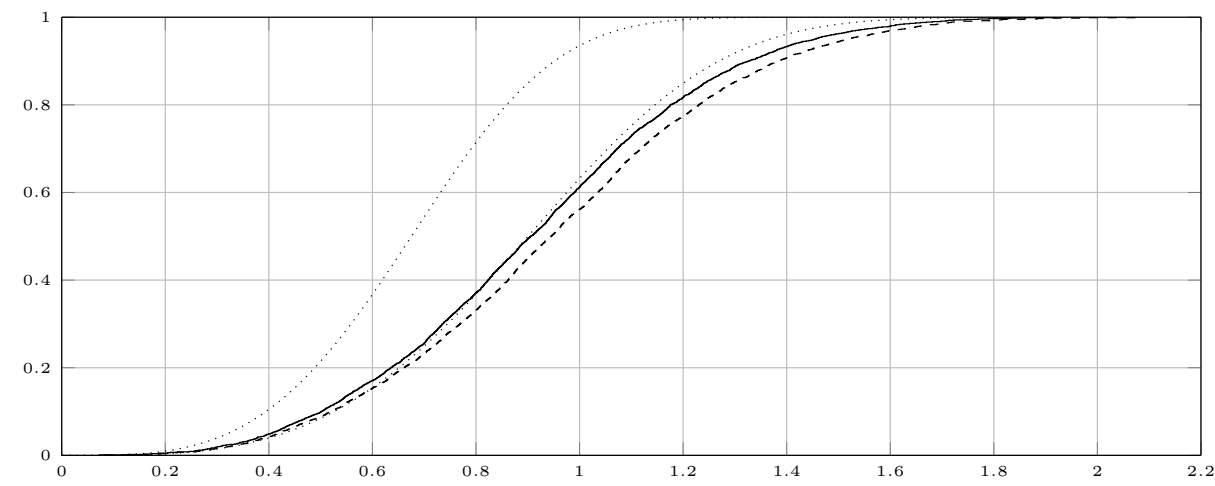

Figure 6.7: Empirical distribution functions of $b_{n}\left(2-M_{n}\right)$ (solid) and $b_{n}\left(2-\bar{M}_{n}\right)$ (dashed) for $d=3$ with $e=2, a_{3}=0.5$ and $n=100000$ (5000 replications). The dotted curves are the bounding functions $G(t)$ (lower) and $G(t /(1-$ $\left.0.5^{2}\right)$ ) (upper).

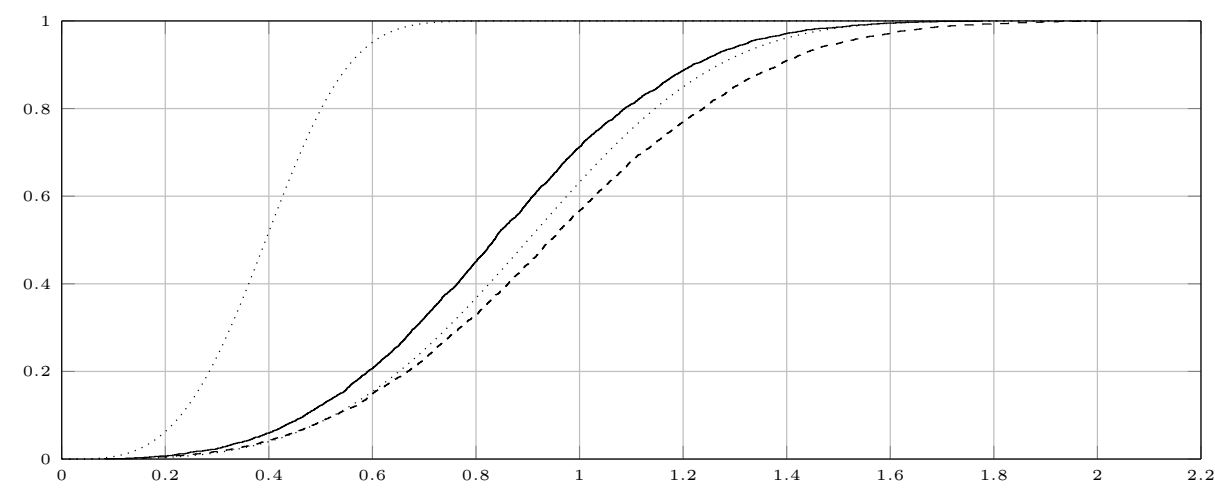

Figure 6.8: Empirical distribution functions of $b_{n}\left(2-M_{n}\right)$ (solid) and $b_{n}\left(2-\bar{M}_{n}\right)$ (dashed) for $d=3$ with $e=2, a_{3}=0.75$ and $n=100000$ (5000 replications). The dotted curves are the bounding functions $G(t)$ (lower) and $G\left(t /\left(1-0.75^{2}\right)\right)$ (upper).

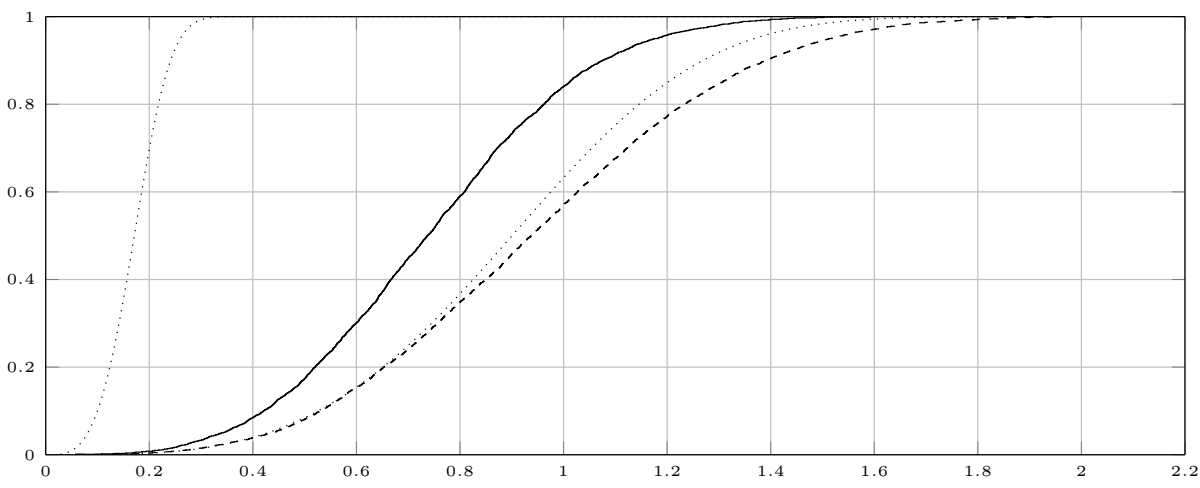

Figure 6.9: Empirical distribution functions of $b_{n}\left(2-M_{n}\right)$ (solid) and $b_{n}\left(2-\bar{M}_{n}\right)$ (dashed) for $d=3$ with $e=2, a_{3}=0.9$ and $n=100000$ (5000 replications). The dotted curves are the bounding functions $G(t)$ (lower) and $G(t /(1-$ $\left.0.9^{2}\right)$ ) (upper). 


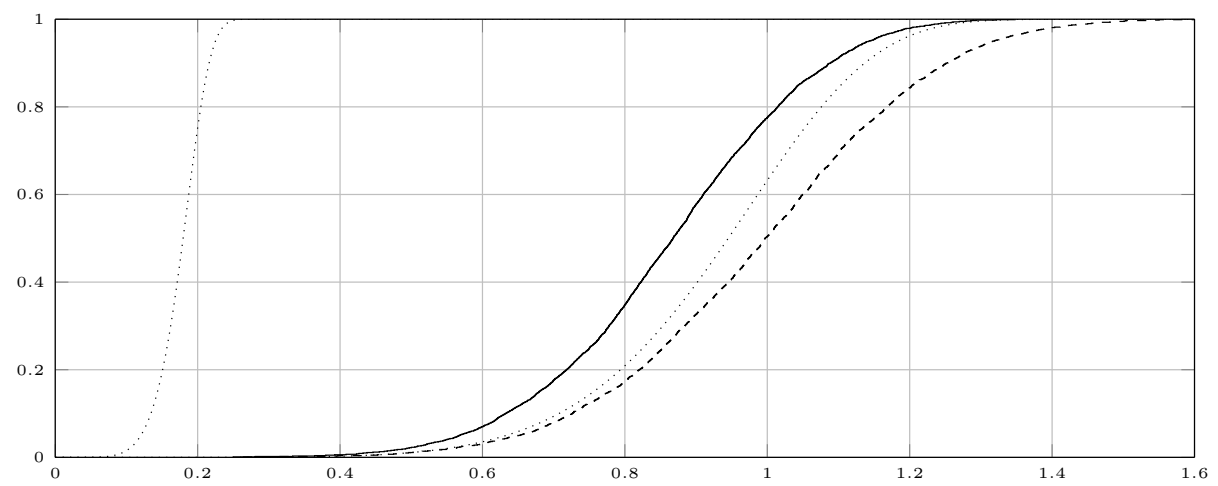

Figure 6.10: Empirical distribution functions of $b_{n}\left(2-M_{n}\right)$ (solid) and $b_{n}\left(2-\bar{M}_{n}\right)$ (dashed) for $d=6$ with $e=2, a_{3}=0.9, a_{4}=a_{5}=a_{6}=0.1$ and $n=100000$ (5000 replications). The dotted curves are the bounding functions $G(t)$ (lower) and $G\left(t /\left(1-0.9^{2}\right)\right)$ (upper).

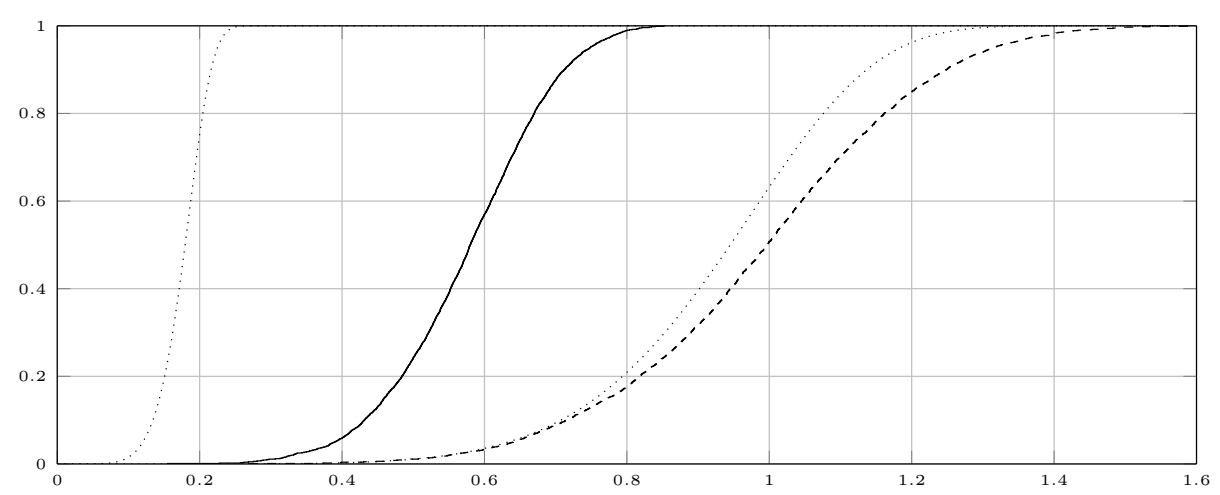

Figure 6.11: Empirical distribution functions of $b_{n}\left(2-M_{n}\right)$ (solid) and $b_{n}\left(2-\bar{M}_{n}\right)$ (dashed) for $d=6$ with $e=2, a_{3}=\ldots=a_{6}=0.9$ and $n=100000(5000$ replications). The dotted curves are the bounding functions $G(t)$ (lower) and $G\left(t /\left(1-0.9^{2}\right)\right)$ (upper).

Proof of Theorem 6.10. From Lemma 6.9 we have

$$
\left|\bar{Z}_{i}-\bar{Z}_{j}\right| \leq\left|Z_{i}-Z_{j}\right| \leq g\left(\bar{Z}_{i}, \bar{Z}_{j}\right)
$$

for all $i, j \in \mathbb{N}$. These inequalities imply

$$
\max _{1 \leq i, j \leq n}\left|\bar{Z}_{i}-\bar{Z}_{j}\right| \leq \max _{1 \leq i, j \leq n}\left|Z_{i}-Z_{j}\right| \leq \max _{1 \leq i, j \leq n} g\left(\bar{Z}_{i}, \bar{Z}_{j}\right)
$$

and thus

$$
\min _{1 \leq i, j \leq n}\left\{2-g\left(\bar{Z}_{i}, \bar{Z}_{j}\right)\right\} \leq \min _{1 \leq i, j \leq n}\left\{2-\left|Z_{i}-Z_{j}\right|\right\} \leq \min _{1 \leq i, j \leq n}\left\{2-\left|\bar{Z}_{i}-\bar{Z}_{j}\right|\right\} .
$$


Using (6.6) and the upper inequality figuring in $(6.8)$ yields

$$
\begin{aligned}
\mathbb{P}\left(b_{n}\left(2-\max _{1 \leq i, j \leq n}\left|Z_{i}-Z_{j}\right|\right) \leq t\right) & =\mathbb{P}\left(2-\max _{1 \leq i, j \leq n}\left|Z_{i}-Z_{j}\right| \leq \frac{t}{b_{n}}\right) \\
& =\mathbb{P}\left(\min _{1 \leq i, j \leq n}\left\{2-\left|Z_{i}-Z_{j}\right|\right\} \leq \frac{t}{b_{n}}\right) \\
& \geq \mathbb{P}\left(\min _{1 \leq i, j \leq n}\left\{2-\left|\bar{Z}_{i}-\bar{Z}_{j}\right|\right\} \leq \frac{t}{b_{n}}\right) \\
& =\mathbb{P}\left(b_{n}\left(2-\max _{1 \leq i, j \leq n}\left|\bar{Z}_{i}-\bar{Z}_{j}\right|\right) \leq t\right) \\
& \rightarrow G(t) .
\end{aligned}
$$

Hence, the lower bound stated in (6.7) has already been obtained. To establish the upper bound in (6.7), we consider $\mathbb{R}^{e} \times \mathbb{R}^{e}$. For $(\bar{x}, \bar{y}) \in \mathbb{B}^{e} \times \mathbb{B}^{e}$ close to $\mathbf{a}:=(-1, \mathbf{0}, 1, \mathbf{0}) \in \mathbb{R}^{2 e}$ we have, putting

$$
c:=1-a_{e+1}^{2},
$$

the multivariate Taylor series expansions

$$
\begin{aligned}
& 2-g(\bar{x}, \bar{y})=c \cdot\left(2+x_{1}-y_{1}\right)+o(|(\bar{x}, \bar{y})-\mathbf{a}|), \\
& 2-|\bar{x}-\bar{y}|=\left(2+x_{1}-y_{1}\right)+o(|(\bar{x}, \bar{y})-\mathbf{a}|),
\end{aligned}
$$

and hence

$$
\frac{2-g(\bar{x}, \bar{y})}{2-|\bar{x}-\bar{y}|}=c+o(|(\bar{x}, \bar{y})-\mathbf{a}|)
$$

By symmetry, we can conclude that

$$
\frac{2-g(\bar{x}, \bar{y})}{2-|\bar{x}-\bar{y}|} \rightarrow c
$$

for $(\bar{x}, \bar{y}) \in \mathbb{B}^{e} \times \mathbb{B}^{e}$ with $(\bar{x}, \bar{y}) \rightarrow\left(\mathbf{a}^{*},-\mathbf{a}^{*}\right)$ and $\mathbf{a}^{*} \in \partial \mathbb{B}^{e}$. Furthermore, the symmetry guarantees that, for each $\delta \in(0, c)$, we can find a positive $\varepsilon$ so that

$$
c-\delta \leq \frac{2-g(\bar{x}, \bar{y})}{2-|\bar{x}-\bar{y}|}
$$

for all $(\bar{x}, \bar{y}) \in \mathbb{B}^{e} \times \mathbb{B}^{e}$ with $|\bar{x}-\bar{y}| \geq 2-\varepsilon$. For $n \in \mathbb{N}$, we write $\bar{Z}_{n}^{1}$ and $\bar{Z}_{n}^{2}$ for 
those elements of $\left\{\bar{Z}_{1}, \ldots, \bar{Z}_{n}\right\}$ with

$$
\max _{1 \leq i, j \leq n}\left|\bar{Z}_{i}-\bar{Z}_{j}\right|=\left|\bar{Z}_{n}^{1}-\bar{Z}_{n}^{2}\right|
$$

Based on these two random variables, we define for $\varepsilon$ given above the set

$$
A_{n, \varepsilon}:=\left\{\left|\bar{Z}_{n}^{1}-\bar{Z}_{n}^{2}\right|>2-\varepsilon\right\}
$$

Obviously, $\mathbb{P}\left(A_{n, \varepsilon}^{c}\right) \rightarrow 0$, and the event $A_{n, \varepsilon}$ entails

$$
c-\delta \leq \frac{2-g\left(\bar{Z}_{n}^{1}, \bar{Z}_{n}^{2}\right)}{2-\left|\bar{Z}_{n}^{1}-\bar{Z}_{n}^{2}\right|}
$$

Together with the lower inequality given in 6.8 we obtain

$$
\begin{aligned}
& \mathbb{P}\left(b_{n}\left(2-\max _{1 \leq i, j \leq n}\left|Z_{i}-Z_{j}\right|\right) \leq t\right) \\
\leq & \mathbb{P}\left(b_{n} \min _{1 \leq i, j \leq n}\left\{2-\left|Z_{i}-Z_{j}\right|\right\} \leq t, A_{n, \varepsilon}\right)+\mathbb{P}\left(A_{n, \varepsilon}^{c}\right) \\
\leq & \mathbb{P}\left(b_{n} \min _{1 \leq i, j \leq n}\left\{2-g\left(\bar{Z}_{i}, \bar{Z}_{j}\right)\right\} \leq t, A_{n, \varepsilon}\right)+\mathbb{P}\left(A_{n, \varepsilon}^{c}\right) \\
= & \mathbb{P}\left(b_{n} \min _{1 \leq i, j \leq n}\left\{\left(2-\left|\bar{Z}_{i}-\bar{Z}_{j}\right|\right) \cdot \frac{2-g\left(\bar{Z}_{i}, \bar{Z}_{j}\right)}{2-\left|\bar{Z}_{i}-\bar{Z}_{j}\right|}\right\} \leq t, A_{n, \varepsilon}\right)+\mathbb{P}\left(A_{n, \varepsilon}^{c}\right) \\
\leq & \mathbb{P}\left(b_{n} \min _{1 \leq i, j \leq n}\left\{\left(2-\left|\bar{Z}_{i}-\bar{Z}_{j}\right|\right) \cdot(c-\delta)\right\} \leq t, A_{n, \varepsilon}\right)+\mathbb{P}\left(A_{n, \varepsilon}^{c}\right) \\
\leq & \mathbb{P}\left(b_{n}\left(2-\max _{1 \leq i, j \leq n}\left|\bar{Z}_{i}-\bar{Z}_{j}\right|\right) \leq \frac{t}{c-\delta}\right)+\mathbb{P}\left(A_{n, \varepsilon}^{c}\right) \\
\rightarrow & G\left(\frac{t}{c-\delta}\right) .
\end{aligned}
$$

Since $\delta$ can be chosen arbitrarily close to 0 , the continuity of $G$ implies

$$
\limsup _{n \rightarrow \infty} \mathbb{P}\left(b_{n}\left(2-\max _{1 \leq i, j \leq n}\left|Z_{i}-Z_{j}\right|\right) \leq t\right) \leq G\left(\frac{t}{c}\right),
$$

and the proof is finished.

\subsubsection{Proofs of Lemma 6.8 And Lemma 6.9}

Proof of Lemma 6.8. Putting

$$
A_{s}:=\operatorname{int}(E) \cap\left\{z \in \mathbb{R}^{d}: 1-s \leq|\bar{z}|\right\}
$$


for $s \in(0,1]$, we have

$$
F(s):=\mathbb{P}\left(1-\left|\bar{Z}_{1}\right| \leq s\right)=\mathbb{P}\left(1-s \leq\left|\bar{Z}_{1}\right|\right)=\int_{A_{s}} f(z) \mathrm{d} z .
$$

In order to compute $F(s)$, we define for fixed $\bar{z} \in \mathbb{B}^{e}$ the set

$$
S(\bar{z}):=\left\{y \in \mathbb{R}^{d-e}:(\bar{z}, y) \in \operatorname{int}(E)\right\}
$$

and the $(d-e) \times(d-e)$-dimensional matrices $\Sigma_{1}:=\operatorname{diag}\left(a_{e+1}^{2}, \ldots, a_{d}^{2}\right)$ and $\Sigma_{2}(\bar{z}):=$ $\left(1-|\bar{z}|^{2}\right) \cdot \Sigma_{1}$. For $z=(\bar{z}, y) \in \operatorname{int}(E)$ the representation $\Sigma=\operatorname{diag}\left(\mathrm{I}_{e}, \Sigma_{1}\right)$ yields

$$
f(z)=c_{1} \cdot\left(1-z^{\top} \Sigma^{-1} z\right)^{\beta}=c_{1} \cdot\left(1-|\bar{z}|^{2}-y^{\top} \Sigma_{1}^{-1} y\right)^{\beta}
$$

and an application of Cavalieri's principle shows that

$$
\begin{aligned}
F(s) & =\int_{\mathbb{B}^{e} \backslash(1-s) \mathbb{B}^{e}}\left(\int_{S(\bar{z})} f(\bar{z}, y) \mathrm{d} y\right) \mathrm{d} \bar{z} \\
& =\int_{\mathbb{B}^{e} \backslash(1-s) \mathbb{B}^{e}}\left(\int_{S(\bar{z})} c_{1} \cdot\left(1-|\bar{z}|^{2}-y^{\top} \Sigma_{1}^{-1} y\right)^{\beta} \mathrm{d} y\right) \mathrm{d} \bar{z} \\
& =c_{1} \cdot \int_{\mathbb{B}^{e} \backslash(1-s) \mathbb{B}^{e}}\left(1-|\bar{z}|^{2}\right)^{\beta}\left(\int_{S(\bar{z})}\left(1-y^{\top} \Sigma_{2}(\bar{z})^{-1} y\right)^{\beta} \mathrm{d} y\right) \mathrm{d} \bar{z} .
\end{aligned}
$$

Rewriting

$$
\begin{aligned}
S(\bar{z}) & =\left\{y \in \mathbb{R}^{d-e}: z_{1}^{2}+\ldots+z_{e}^{2}+\left(\frac{y_{1}}{a_{e+1}}\right)^{2}+\ldots+\left(\frac{y_{d-e}}{a_{d}}\right)^{2}<1\right\} \\
& =\left\{y \in \mathbb{R}^{d-e}:\left(\frac{y_{1}}{a_{e+1}}\right)^{2}+\ldots+\left(\frac{y_{d-e}}{a_{d}}\right)^{2}<1-|\bar{z}|^{2}\right\} \\
& =\left\{y \in \mathbb{R}^{d-e}:\left(\frac{y_{1}}{a_{e+1} \sqrt{1-|\bar{z}|^{2}}}\right)^{2}+\ldots+\left(\frac{y_{d-e}}{a_{d} \sqrt{1-|\bar{z}|^{2}}}\right)^{2}<1\right\}
\end{aligned}
$$

reveals that $S(\bar{z})$ is a $(d-e)$-dimensional ellipsoid, and using the matrix $\Sigma_{2}(\bar{z})$, we can write

$$
S(\bar{z})=\left\{y \in \mathbb{R}^{d-e}: y^{\top} \Sigma_{2}(\bar{z})^{-1} y<1\right\}
$$


This description of the set $S(\bar{z})$ makes it clear that the function

$$
\left(1-y^{\top} \Sigma_{2}(\bar{z})^{-1} y\right)^{\beta} \cdot \mathbb{1}\{y \in S(\bar{z})\}
$$

occurring in (6.9), is, up to a scaling factor, the density of an appropriately defined Pearson Type II distribution in $d-e$ dimensions. The missing scaling factor to obtain a probability density is given by

$$
c_{2}(\bar{z})=\frac{\Gamma\left(\frac{d-e}{2}+\beta+1\right)}{\Gamma(\beta+1) \pi^{\frac{d-e}{2}} \prod_{i=e+1}^{d}\left(a_{i} \sqrt{1-|\bar{z}|^{2}}\right)},
$$

see Example 5.4. Writing

$$
c_{3}:=\frac{\Gamma\left(\frac{d-e}{2}+\beta+1\right)}{\Gamma(\beta+1) \pi^{\frac{d-e}{2}} \prod_{i=e+1}^{d} a_{i}},
$$

we have

$$
c_{2}(\bar{z})=c_{3} \cdot\left(1-|\bar{z}|^{2}\right)^{-\frac{d-e}{2}}
$$

and $(6.9)$ can be written as

$$
\begin{aligned}
F(s) & =c_{1} \cdot \int_{\mathbb{B}^{e} \backslash(1-s) \mathbb{B}^{e}}\left(1-|\bar{z}|^{2}\right)^{\beta}\left(\int_{S(\bar{z})}\left(1-y^{\top} \Sigma_{2}(\bar{z})^{-1} y\right)^{\beta} \mathrm{d} y\right) \mathrm{d} \bar{z} \\
& =c_{1} \cdot \int_{\mathbb{B}^{e} \backslash(1-s) \mathbb{B}^{e}}\left(1-|\bar{z}|^{2}\right)^{\beta} c_{2}(\bar{z})^{-1} \mathrm{~d} \bar{z} \\
& =c_{1} \cdot \int_{\mathbb{B}^{e} \backslash(1-s) \mathbb{B}^{e}}\left(1-|\bar{z}|^{2}\right)^{\beta} \frac{1}{c_{3}}\left(1-|\bar{z}|^{2}\right)^{\frac{d-e}{2}} \mathrm{~d} \bar{z} \\
& =\frac{c_{1}}{c_{3}} \cdot \int_{\mathbb{B}^{e} \backslash(1-s) \mathbb{B}^{e}}\left(1-|\bar{z}|^{2}\right)^{\beta+\frac{d-e}{2}} \mathrm{~d} \bar{z} .
\end{aligned}
$$

Using e-dimensional spherical coordinates yields

$$
\begin{aligned}
\int_{\mathbb{B}^{e} \backslash(1-s) \mathbb{B}^{e}}\left(1-|\bar{z}|^{2}\right)^{\frac{d-e}{2}+\beta} \mathrm{d} \bar{z} & =\int_{\mathcal{S}^{e-1}} \int_{1-s}^{1}\left(1-r^{2}\right)^{\frac{d-e}{2}+\beta} r^{e-1} \mathrm{~d} r \mathcal{H}^{e-1}(\mathrm{~d} u) \\
& =\int_{\mathcal{S}^{e-1}} 1 \mathcal{H}^{e-1}(\mathrm{~d} u) \cdot \int_{1-s}^{1}\left(1-r^{2}\right)^{\frac{d-e}{2}+\beta} r^{e-1} \mathrm{~d} r
\end{aligned}
$$


and since the surface area of $\mathcal{S}^{e-1}$ is $e \cdot \omega_{e}$, we obtain

$$
\int_{\mathbb{B}^{e} \backslash(1-s) \mathbb{B}^{e}}\left(1-|\bar{z}|^{2}\right)^{\frac{d-e}{2}+\beta} \mathrm{d} \bar{z}=e \cdot \omega_{e} \cdot \int_{1-s}^{1}\left(1-r^{2}\right)^{\frac{d-e}{2}+\beta} r^{e-1} \mathrm{~d} r
$$

Putting (6.10) and (6.11) together and observing

$$
\frac{c_{1}}{c_{3}}=\frac{\Gamma\left(\frac{d}{2}+\beta+1\right)}{\Gamma(\beta+1) \pi^{\frac{d}{2}} \prod_{i=e+1}^{d} a_{i}} \cdot \frac{\Gamma(\beta+1) \pi^{\frac{d-e}{2}} \prod_{i=e+1}^{d} a_{i}}{\Gamma\left(\frac{d-e}{2}+\beta+1\right)}=\frac{\Gamma\left(\frac{d}{2}+\beta+1\right)}{\Gamma\left(\frac{d-e}{2}+\beta+1\right)} \cdot \pi^{-\frac{e}{2}}
$$

yields

$$
F(s)=\frac{\Gamma\left(\frac{d}{2}+\beta+1\right)}{\Gamma\left(\frac{d-e}{2}+\beta+1\right)} \cdot \pi^{-\frac{e}{2}} \cdot e \cdot \omega_{e} \int_{1-s}^{1}\left(1-r^{2}\right)^{\frac{d-e}{2}+\beta} r^{e-1} \mathrm{~d} r .
$$

This representation reveals that the distribution of $\left|\bar{Z}_{1}\right|$ does not depend on the lengths $a_{e+1}, \ldots, a_{d}$ of the half-axes of $E$. As stated at the beginning of this section, the same result holds true for the distribution of $\bar{Z}_{1}$ itself, since $\bar{Z}_{1}$ has a spherically symmetric distribution in $\mathbb{B}^{e}$. For calculating the integral above, we substitute $r=1-t$ and use

$$
\left(2 t-t^{2}\right)^{\frac{d-e}{2}+\beta}(1-t)^{e-1}=(2 t)^{\frac{d-e}{2}+\beta}+O\left(t^{\frac{d-e}{2}+\beta+1}\right)
$$

as $t \downarrow 0$ to get

$$
\begin{aligned}
\int_{1-s}^{1}\left(1-r^{2}\right)^{\frac{d-e}{2}+\beta} r^{e-1} \mathrm{~d} r & =\int_{s}^{0}\left(2 t-t^{2}\right)^{\frac{d-e}{2}+\beta}(1-t)^{e-1}(-1) \mathrm{d} t \\
& =\int_{0}^{s}\left(2 t-t^{2}\right)^{\frac{d-e}{2}+\beta}(1-t)^{e-1} \mathrm{~d} t \\
& =\int_{0}^{s}\left((2 t)^{\frac{d-e}{2}+\beta}+O\left(t^{\frac{d-e}{2}+\beta+1}\right)\right) \mathrm{d} t \\
& =\frac{2^{\frac{d-e}{2}}+\beta}{\frac{d-e}{2}+\beta+1} \cdot s^{\frac{d-e}{2}+\beta+1}+O\left(s^{\frac{d-e}{2}+\beta+2}\right) .
\end{aligned}
$$


With the definitions of $a$ and $\alpha$ on page 129 , we can conclude from $(6.12)$ that

$$
\begin{aligned}
F(s) & =\frac{\Gamma\left(\frac{d}{2}+\beta+1\right)}{\Gamma\left(\frac{d-e}{2}+\beta+1\right)} \cdot \pi^{-\frac{e}{2}} \cdot e \cdot \omega_{e} \cdot \frac{2^{\frac{d-e}{2}+\beta}}{\frac{d-e}{2}+\beta+1} \cdot s^{\frac{d-e}{2}+\beta+1}+O\left(s^{\frac{d-e}{2}+\beta+2}\right) \\
& =\frac{\Gamma\left(\frac{d}{2}+\beta+1\right)}{\Gamma\left(\frac{d-e}{2}+\beta+2\right)} \cdot \pi^{-\frac{e}{2}} \cdot e \cdot \omega_{e} \cdot 2^{\frac{d-e}{2}+\beta} \cdot s^{\frac{d-e}{2}+\beta+1}+O\left(s^{\frac{d-e}{2}+\beta+2}\right) \\
& =a s^{\alpha}+O\left(s^{\alpha+1}\right) \\
& \sim a s^{\alpha}
\end{aligned}
$$

as $s \downarrow 0$.

Proof of Lemma 6.9. Since, for each $z \in E$, we have the inequality

$$
\left|\left(z_{e+1}, \ldots, z_{d}\right)\right| \leq a_{e+1} \sqrt{1-z_{1}^{2}-\ldots-z_{e}^{2}}=a_{e+1} \sqrt{1-|\bar{z}|^{2}}
$$

we get for $x, y \in E$

$$
\begin{aligned}
\left(x_{e+1}-y_{e+1}\right)^{2}+\ldots+\left(x_{d}-y_{d}\right)^{2} & =\left|\left(x_{e+1}-y_{e+1}, \ldots, x_{d}-y_{d}\right)\right|^{2} \\
& \leq\left(\left|\left(x_{e+1}, \ldots, x_{d}\right)\right|+\left|\left(y_{e+1}, \ldots, y_{d}\right)\right|\right)^{2} \\
& \leq\left(a_{e+1} \sqrt{1-|\bar{x}|^{2}}+a_{e+1} \sqrt{1-|\bar{y}|^{2}}\right)^{2} \\
& =a_{e+1}^{2}\left(\sqrt{1-|\bar{x}|^{2}}+\sqrt{1-|\bar{y}|^{2}}\right)^{2} \\
& \leq 2 a_{e+1}^{2}\left(1-|\bar{x}|^{2}+1-|\bar{y}|^{2}\right) \\
& =2 a_{e+1}^{2}\left(2-|\bar{x}|^{2}-|\bar{y}|^{2}\right) .
\end{aligned}
$$

Observe that the last inequality holds due to the convexity of $z \mapsto z^{2}$ : For $a, b \in \mathbb{R}$ we have

$$
(a+b)^{2}=4\left(\frac{1}{2} a+\frac{1}{2} b\right)^{2} \leq 4\left(\frac{1}{2} a^{2}+\frac{1}{2} b^{2}\right)=2\left(a^{2}+b^{2}\right) .
$$

Finally, the inequalities above show

$$
\begin{aligned}
|\bar{x}-\bar{y}|^{2} & \leq|x-y|^{2} \\
& =\left(x_{1}-y_{1}\right)^{2}+\ldots+\left(x_{e}-y_{e}\right)^{2}+\left(x_{e+1}-y_{e+1}\right)^{2}+\ldots+\left(x_{d}-y_{d}\right)^{2} \\
& =|\bar{x}-\bar{y}|^{2}+\left(x_{e+1}-y_{e+1}\right)^{2}+\ldots+\left(x_{d}-y_{d}\right)^{2} \\
& \leq(|\bar{x}|+|\bar{y}|)^{2}+2 a_{e+1}^{2}\left(2-|\bar{x}|^{2}-|\bar{y}|^{2}\right) .
\end{aligned}
$$


Appendices 



\section{PRINCIPAL CURVATURES AND DIRECTIONS}

This appendix lists some basics about the curvature of hypersurfaces. For this purpose, we will mainly use the notation of Csikós [5].

\section{A.1 General theory}

\section{A.1.1 The CURVATURe of Planar Curves}

According to Gray [9, p. 4], a curve in $\mathbb{R}^{2}$ is a function $\alpha$ that maps some open interval $(a, b)$ into $\mathbb{R}^{2}$, having partial derivatives of all orders. It is often assumed that a curve possesses partial derivatives of all orders, but we only need first- and second-order partial derivatives. We call a curve regular if the speed vector $\alpha^{\prime}(t)$ is nonzero for each $t \in(a, b)$. Writing $J: \mathbb{R}^{2} \rightarrow \mathbb{R}^{2}, J\left(z_{1}, z_{2}\right):=\left(-z_{2}, z_{1}\right)$ for the rotation by $\pi / 2$ in a counterclockwise direction, the curvature of a regular curve $\alpha:(a, b) \rightarrow \mathbb{R}^{2}$ at $t$ is defined as

$$
\kappa(t):=\frac{\alpha^{\prime \prime}(t) \cdot J\left(\alpha^{\prime}(t)\right)}{\left|\alpha^{\prime}(t)\right|^{3}}
$$

and the representation $\alpha(t)=(x(t), y(t))$ yields

$$
\kappa(t)=\frac{x^{\prime}(t) y^{\prime \prime}(t)-x^{\prime \prime}(t) y^{\prime}(t)}{\left(x^{\prime}(t)^{2}+y^{\prime}(t)^{2}\right)^{\frac{3}{2}}} .
$$


An easy interpretation of the curvature $\kappa$ is given as follows: If $\alpha^{\prime \prime}(t) \neq 0$, the radius of the osculating circle to $\alpha$ at the point $\alpha(t)$ is $|\kappa(t)|^{-1}$, see Figure A.1 for an illustration. So, a circle with radius $r>0$ has constant curvature $1 / r$ resp. $-1 / r$,

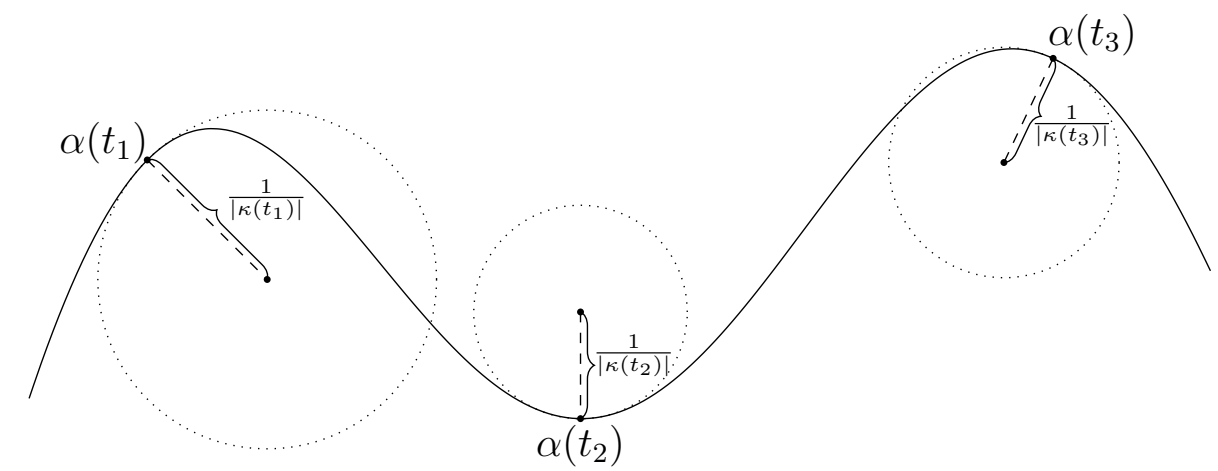

Figure A.1: A planar curve $\alpha$ and the osculating circles at three different points.

depending on the direction of the chosen parametrization. For some more details on the curvature of planar curves, see for example Gray [9, p. 1-16].

\section{A.1.2 The CurVature of HyPersurfaces}

For example, some of the basic definitions and facts about the curvature of hypersurfaces as needed in this thesis are given in Schneider [22, p. 112-115], Aminov [2, p. 31-34] or Lee [18, p. 139-141]. But, since it turned out to fit best for our purposes, we mainly follow the notation of Csikós [5, p. 141-150]. In that work, only functions that are differentiable infinitely often are called smooth. But since we only need first- and second-order derivatives, we will call a function smooth, whenever it is twice continuously differentiable. Here and in A.2.1, we will slightly differ from our notation for partial derivatives, given at the beginning of Section 2.1. If $s$ is a function, that maps some open subset of $\mathbb{R}^{d-1}$ into $\mathbb{R}$, we will write $s_{i}$ and $s_{i j}$ for the first- and the second-order partial derivatives with respect to the $i$-th and $j$-th variable, $i, j \in\{1, \ldots, d-1\}$. In the same way we write $\mathbf{s}_{i}$ and $\mathbf{s}_{i j}$ for the $d$-dimensional vectors of all first- and second-order partial derivatives with respect to the $i$-th and the $j$-th variable, if $\mathbf{s}$ is a function, that maps from some open subset of $\mathbb{R}^{d-1}$ into $\mathbb{R}^{d}, i, j \in\{1, \ldots, d-1\}$.

Definition A.1. A smooth parameterized hypersurface in $\mathbb{R}^{d}$ is a smooth mapping $\mathbf{r}: O \rightarrow \mathbb{R}^{d}$, where $O \subset \mathbb{R}^{d-1}$ is open. We call $\mathbf{r}$ regular if the vectors $\mathbf{r}_{1}(u), \ldots, \mathbf{r}_{d-1}(u)$ are linearly independent for each $u \in O$, and we write $M$ for the image $\mathbf{r}(O)$ of the hypersurface. 
Definition A.2. For $u \in O$, the (linear) tangent space of a smooth parameterized and regular hypersurface $\mathbf{r}: O \rightarrow \mathbb{R}^{d}$ at the point $p=\mathbf{r}(u) \in M$ is the linear space $T_{p} M$, spanned by the vectors $\mathbf{r}_{1}(u), \ldots, \mathbf{r}_{d-1}(u)$. The unit normal vector of the hypersurface at the point $\mathbf{r}(u)$ is defined as that unit normal vector $\mathbf{N}(u)$ of $T_{p} M$ for which $\left\{\mathbf{r}_{1}(u), \ldots, \mathbf{r}_{d-1}(u), \mathbf{N}(u)\right\}$ is a positively oriented basis of $\mathbb{R}^{d}$.

Observe that this choice of $\mathbf{N}(u)$ is arbitrary. Choosing the other unit normal vector would only result in different signs of the principal curvatures, to be defined later. One way to study the curvature of a smooth parameterized and regular hypersurface $\mathbf{r}: O \rightarrow \mathbb{R}^{d}$ at the point $p=\mathbf{r}\left(u_{0}\right), u_{0} \in O$, is to investigate the curvatures of curves lying on $M$, passing through $p$. For this purpose, let $v \neq 0$ be an arbitrary tangent vector of $\mathbf{r}$ at $p=\mathbf{r}\left(u_{0}\right)$. The curve of intersection of $M$ and the plane $\mathbf{r}\left(u_{0}\right)+\operatorname{span}\left\{v, \mathbf{N}\left(u_{0}\right)\right\}$ is called the normal section of the hypersurface in the direction of $v$. We choose such a parametrization of this curve of intersection, so that its speed vector at $p$ is given by the chosen tangent vector $v$. Such a choice is always possible in a sufficiently small neighborhood of $p$. By orienting the cutting normal plane by the ordered basis $\left\{v, \mathbf{N}\left(u_{0}\right)\right\}$, we may consider the signed curvature of the normal section, which will be called the normal curvature of the hypersurface in the direction of $v$ and will be denoted by $k(v)$. Formally, this curvature can be computed as stated in A.1.1. An easier way is as follows: Given some tangent vector $v=v_{1} \mathbf{r}_{1}\left(u_{0}\right)+\ldots+v_{d-1} \mathbf{r}_{d-1}\left(u_{0}\right)$, the normal curvature in this direction can be computed as

$$
k(v)=\frac{1}{|v|^{2}} \sum_{i, j=1}^{d-1}\left\langle\mathbf{N}\left(u_{0}\right), \mathbf{r}_{i j}\left(u_{0}\right)\right\rangle v_{i} v_{j},
$$

see equation (3.2) in Csikós [5]. Obviously, $k(\lambda v)=k(v)$ holds true for all $\lambda \neq 0$, so that the normal curvature depends only on the direction of $v$. It would be of course completely impracticable to describe the curvature of $M$ at a given point $p=\mathbf{r}\left(u_{0}\right)$ via all possible normal curvatures at this point. Fortunately, there is a much easier way: The complete information of curvature of $M$ at a given point $p=\mathbf{r}\left(u_{0}\right)$ is given by $d-1$ numbers and $d-1$ vectors: the principal curvatures and principal curvature directions. But before we can define the principal curvatures as the eigenvalues of the Weingarten map, we need to define the derivative of a function $X: O \rightarrow \mathbb{R}^{d}$ with respect to some tangent vector of the hypersurface $\mathbf{r}$.

Definition A.3. Let $\mathbf{r}: O \rightarrow \mathbb{R}^{d}$ be a smooth parameterized and regular hypersurface, $X: O \rightarrow \mathbb{R}^{d}, u_{0} \in O$ and $v$ a tangent vector of $\mathbf{r}$ at the point $\mathbf{r}\left(u_{0}\right)$. The derivative $\partial_{v} X$ of $X$ with respect to the tangent vector $v$ is defined as

$$
\partial_{v} X:=(X \circ \mathbf{u})^{\prime}(0)
$$


where $\mathbf{u}:(-1,1) \rightarrow O$ is a curve within the parameter domain $O$, fulfilling $\mathbf{u}(0)=u_{0}$ and $(\mathbf{r} \circ \mathbf{u})^{\prime}(0)=v$.

Now we are able to introduce the Weingarten map:

Definition A.4. Let $\mathbf{r}: O \rightarrow \mathbb{R}^{d}$ be a smooth parameterized and regular hypersurface and $M=\mathbf{r}(O)$. For $u_{0} \in O$ and $p=\mathbf{r}\left(u_{0}\right)$, the linear map

$$
L_{p}:\left\{\begin{array}{l}
T_{p} M \rightarrow T_{p} M, \\
v \mapsto-\partial_{v} \mathbf{N}
\end{array}\right.
$$

is called the Weingarten map or shape operator of $M$ at $p$.

Definition A.5. Let $\mathbf{r}: O \rightarrow \mathbb{R}^{d}$ be a smooth parameterized and regular hypersurface, $M=\mathbf{r}(O), u_{0} \in O, T_{p} M$ the tangent space of $M$ at $p=\mathbf{r}\left(u_{0}\right)$ and $L_{p}: T_{p} M \rightarrow T_{p} M$ the Weingarten map. The restriction of the scalar product to the tangent space $T_{p} M$ leads to a bilinear function

$$
I_{p}(v, w):=\langle v, w\rangle, \quad v, w \in T_{p} M
$$

the so-called first fundamental form of the hypersurface. The second fundamental form of the hypersurface is the bilinear function $I I_{p}$ on $T_{p} M$, given by

$$
I I_{p}(v, w):=\left\langle L_{p} v, w\right\rangle, \quad v, w \in T_{p} M
$$

By use of the first and the second fundamental form, we can compute the normal curvature of the hypersurface at the point $p \in M$ in direction $v$ via

$$
k(v)=\frac{I I_{p}(v, v)}{I_{p}(v, v)}
$$

see Csikós [5, p. 147]. This representation of the normal curvature reveals that, for each $p \in M$, there are directions $v_{1}, v_{d-1} \in T_{p} M$ so that $k\left(v_{1}\right) \leq k(v) \leq k\left(v_{d-1}\right)$ for all $v \in T_{p} M$. These bounds follow from the fact that the first and second fundamental forms are continuous, and because of $k(\lambda v)=k(v)$ for each $\lambda \neq 0$, it is enough to consider the compact set $\left\{v \in T_{p} M:|v|=1\right\}$, on which $k$ attains its maximum and minimum. For $d=3$, Euler's formula shows that the minimum $k\left(v_{1}\right)$ and the maximum $k\left(v_{2}\right)$ of all normal curvatures at a point $p$ combined with the corresponding vectors $v_{1}$ and $v_{2}$ contain the complete information of curvature of the hypersurface $\mathbf{r}$ at $p$. A generalized version (for any dimension $d$ ) of this formula can be found below. It is also well-known that the directions $v_{1}$ and $v_{d-1}$ are necessarily orthogonal to 
each other if $k\left(v_{1}\right) \neq k\left(v_{d-1}\right)$. To describe the complete information of curvature of a hypersurface $\mathbf{r}$ in higher dimensions at a point $p$, we need additional characteristic values and directions of the hypersurface at the given point $p$ : the $d-1$ eigenvalues and eigenvectors of the Weingarten map. Theorem 3.1.11 in Csikós [5] shows that

$$
\left\langle L_{p} v, w\right\rangle=\left\langle v, L_{p} w\right\rangle
$$

holds true for all $v, w \in T_{p} M$. In other words, the Weingarten map $L_{p}$ is self-adjoint with respect to the first fundamental form. By applying the principal axis theorem (Theorem 1.2.65 in Csikós [5]), we can conclude that there is an orthonormal basis of the tangent space $T_{p} M$, consisting of eigenvectors of the Weingarten map $L_{p}$.

Definition A.6. Let $\mathbf{r}: O \rightarrow \mathbb{R}^{d}$ be a smooth parameterized and regular hypersurface, $M=\mathbf{r}(O), u_{0} \in O$ and $p=\mathbf{r}\left(u_{0}\right)$. Then the eigenvalues $\kappa_{1} \leq \ldots \leq \kappa_{d-1}$ of the Weingarten map $L_{p}: T_{p} M \rightarrow T_{p} M$ are called the principal curvatures of $M$ at $p$. The corresponding eigenvectors of length 1 are called the principal curvature directions.

The reasoning above demonstrates that we can always find $d-1$ principal curvature directions $v_{1}, \ldots, v_{d-1}$, corresponding to $\kappa_{1}, \ldots, \kappa_{d-1}$, in such a way that they form an orthonormal basis of $T_{p} M$. Notice that $\left\langle v_{i}, v_{j}\right\rangle=0$ is given naturally if $\kappa_{i} \neq \kappa_{j}$. The following result justifies the assertion stated before, that the principal curvatures together with the corresponding directions contain the complete information about the curvatures of the hypersurface at a given point:

Theorem A.7 (Euler's formula). Let $\left\{v_{1}, \ldots, v_{d-1}\right\}$ be an orthonormal basis of $T_{p} M$, consisting of principal curvature directions with respect to the principal curvatures $\kappa_{1}, \ldots, \kappa_{d-1}$. Then, the normal curvature $k(v)$ in the direction of $v \in T_{p} M,|v|=1$, is given by

$$
k(v)=\sum_{i=1}^{d-1} \kappa_{i}\left\langle v, v_{i}\right\rangle^{2}=\sum_{i=1}^{d-1} \kappa_{i} \cos ^{2}\left(\theta_{i}\right),
$$

where $\theta_{i}=\arccos \left(\left\langle v, v_{i}\right\rangle\right), i \in\{1, \ldots, d-1\}$, is the angle between $v$ and $v_{i}$.

Proof. See Csikós [5, Theorem 3.1.16].

\section{A.2 Calculation of principal Curvatures}

\section{A.2.1 Calculation For General hypersurfaces}

For calculating the principal curvatures and directions of a hypersurface $\mathbf{r}$ at a given point $p=\mathbf{r}\left(u_{0}\right)$, it is very useful to consider a matrix representation of the Weingarten 
map $L_{p}$. The most natural way is to use this representation with respect to the basis $\left\{\mathbf{r}_{1}\left(u_{0}\right), \ldots, \mathbf{r}_{d-1}\left(u_{0}\right)\right\}$ of the tangent space at $p=\mathbf{r}\left(u_{0}\right)$. Like Csikós [5, p. 149], we write $\mathcal{G}\left(u_{0}\right)=\left(g_{i j}\left(u_{0}\right)\right)_{1 \leq i, j \leq d-1}$ resp. $\mathcal{B}\left(u_{0}\right)=\left(b_{i j}\left(u_{0}\right)\right)_{1 \leq i, j \leq d-1}$ for the matrix representations of the first resp. second fundamental form with respect to this basis and $\mathcal{L}\left(u_{0}\right)=\left(\ell_{i j}\left(u_{0}\right)\right)_{1 \leq i, j \leq d-1}$ for that of the Weingarten map $L_{p}$. The components of $\mathcal{G}\left(u_{0}\right)$ and $\mathcal{B}\left(u_{0}\right)$ can be calculated according to

$$
\begin{aligned}
& g_{i j}\left(u_{0}\right)=\left\langle\mathbf{r}_{i}\left(u_{0}\right), \mathbf{r}_{j}\left(u_{0}\right)\right\rangle, \\
& b_{i j}\left(u_{0}\right)=\left\langle L_{p} \mathbf{r}_{i}\left(u_{0}\right), \mathbf{r}_{j}\left(u_{0}\right)\right\rangle=\left\langle\mathbf{N}\left(u_{0}\right), \mathbf{r}_{i j}\left(u_{0}\right)\right\rangle,
\end{aligned}
$$

$1 \leq i, j \leq d-1$, see Lemma 3.1.12 in Csikós [5] for the last equality. It can be shown that the matrix representation $\mathcal{L}\left(u_{0}\right)$ of the Weingarten map with respect to the basis $\left\{\mathbf{r}_{1}\left(u_{0}\right), \ldots, \mathbf{r}_{d-1}\left(u_{0}\right)\right\}$ is given by $\mathcal{L}\left(u_{0}\right)=\mathcal{G}\left(u_{0}\right)^{-1} \mathcal{B}\left(u_{0}\right)$, see Csikós [5, p. 150]. Thus, the principal curvatures $\kappa_{1} \leq \ldots \leq \kappa_{d-1}$ of a hypersurface $\mathbf{r}$ at $\mathbf{r}\left(u_{0}\right)$ are exactly the ordered eigenvalues of the matrix $\mathcal{G}\left(u_{0}\right)^{-1} \mathcal{B}\left(u_{0}\right)$. The corresponding eigenvectors are the representations of the principal curvature directions with respect to the basis $\left\{\mathbf{r}_{1}\left(u_{0}\right), \ldots, \mathbf{r}_{d-1}\left(u_{0}\right)\right\}$, i.e. if $z=\left(z_{1}, \ldots, z_{d-1}\right)$ is an eigenvector of $\mathcal{L}\left(u_{0}\right)$, the corresponding principal curvature direction is

$$
v=\sum_{j=1}^{d-1} z_{j} \cdot \mathbf{r}_{j}\left(u_{0}\right) .
$$

\section{A.2.2 Calculation in our Setting}

In this subsection we apply the results of A.2.1 to the setting given in Section 3.1, where the image $M$ of the hypersurface is the graph of a function $s$, that maps from an open subset of $\mathbb{R}^{d-1}$ into $\mathbb{R}$. For this purpose, let $O \subset \mathbb{R}^{d-1}$ be an open subset and $s: O \rightarrow \mathbb{R}$ a twice continuously differentiable function. Like in Section 3.1, points lying in $O$ will be written as $\widetilde{z}=\left(z_{2}, \ldots, z_{d}\right)$, and we define the hypersurface

$$
\mathbf{s}:\left\{\begin{array}{l}
O \rightarrow \mathbb{R}^{d}, \\
\widetilde{z} \mapsto(s(\widetilde{z}), \widetilde{z}) .
\end{array}\right.
$$

As stated in Remark 3.1, we have expressed the first component of $\mathbf{s}$ in terms of the last $d-1$ components to emphasize the very special role of this component in our main theorem. Because of this convention, and in contrast to A.1.2 and A.2.1, we will now use again the notation of partial derivatives as introduced at the very beginning of this thesis: The first- and the second-order partial derivatives of $s$ with respect to $z_{i}$ and $z_{j}$ will be denoted by $s_{i}$ and $s_{i j}$, respectively, $i, j \in\{2, \ldots, d\}$. We 
get

$$
\mathbf{s}_{2}(\widetilde{z})=\left(s_{2}(\widetilde{z}), 1,0, \ldots, 0\right) \quad, \quad \ldots \quad, \quad \mathbf{s}_{d}(\widetilde{z})=\left(s_{d}(\widetilde{z}), 0, \ldots, 0,1\right)
$$

and

$$
\mathbf{s}_{i j}(\widetilde{z})=\left(s_{i j}(\widetilde{z}), 0, \ldots, 0\right)
$$

for $i, j \in\{2, \ldots, d\}$. Briefly written we have

$$
\mathbf{s}_{i}(\widetilde{z})=s_{i}(\widetilde{z}) \cdot \mathbf{e}_{1}+\mathbf{e}_{i} \quad \text { and } \quad \mathbf{s}_{i j}(\widetilde{z})=s_{i j}(\widetilde{z}) \cdot \mathbf{e}_{1}
$$

for $i, j \in\{2, \ldots, d\}$.

Lemma A.8. For each $\widetilde{z} \in O$, the two unit normal vectors of the hypersurface $\mathbf{s}$ at the point $\mathbf{s}(\widetilde{z})$ are given by

$$
\mathbf{N}(\widetilde{z})= \pm \frac{1}{\sqrt{1+\sum_{j=2}^{d} s_{j}(\widetilde{z})^{2}}} \cdot\left(\mathbf{e}_{1}-\sum_{j=2}^{d} s_{j}(\widetilde{z}) \cdot \mathbf{e}_{j}\right)
$$

Proof. Let $\widetilde{z} \in O$ be arbitrary. We have to show that $\mathbf{N}(\widetilde{z})$ is orthogonal to the tangent space of $\mathbf{s}$ at the point $\mathbf{s}(\widetilde{z})$. A basis of this tangent space is given by $\left\{\mathbf{s}_{2}(\widetilde{z}), \ldots, \mathbf{s}_{d}(\widetilde{z})\right\}$, and for $i \in\{2, \ldots, d\}$ we obtain

$$
\begin{aligned}
& \left\langle\mathbf{e}_{1}-\sum_{j=2}^{d} s_{j}(\widetilde{z}) \cdot \mathbf{e}_{j}, \mathbf{s}_{i}(\widetilde{z})\right\rangle \\
= & \left\langle\mathbf{e}_{1}-\sum_{j=2}^{d} s_{j}(\widetilde{z}) \cdot \mathbf{e}_{j}, s_{i}(\widetilde{z}) \cdot \mathbf{e}_{1}+\mathbf{e}_{i}\right\rangle \\
= & s_{i}(\widetilde{z})\left\langle\mathbf{e}_{1}, \mathbf{e}_{1}\right\rangle+\left\langle\mathbf{e}_{1}, \mathbf{e}_{i}\right\rangle-\sum_{j=2}^{d} s_{j}(\widetilde{z}) s_{i}(\widetilde{z})\left\langle\mathbf{e}_{j}, \mathbf{e}_{1}\right\rangle-\sum_{j=2}^{d} s_{j}(\widetilde{z})\left\langle\mathbf{e}_{j}, \mathbf{e}_{i}\right\rangle \\
= & s_{i}(\widetilde{z})+0-\sum_{j=2}^{d} s_{j}(\widetilde{z}) s_{i}(\widetilde{z}) \cdot 0-\sum_{j=2}^{d} s_{j}(\widetilde{z}) \cdot \delta_{i j} \\
= & s_{i}(\widetilde{z})-s_{i}(\widetilde{z}) \\
= & 0 .
\end{aligned}
$$

Scaling finishes the proof. 
According to A.2.1, we define the two $(d-1) \times(d-1)$-dimensional matrices $\mathcal{G}(\widetilde{z})=\left(g_{i j}(\widetilde{z})\right)_{i, j=2, \ldots, d}$ and $\mathcal{B}(\widetilde{z})=\left(b_{i j}(\widetilde{z})\right)_{i, j=2, \ldots, d}$ by

$$
g_{i j}(\widetilde{z}):=\left\langle\mathbf{s}_{i}(\widetilde{z}), \mathbf{s}_{j}(\widetilde{z})\right\rangle= \begin{cases}1+s_{i}(\widetilde{z})^{2} & , i=j, \\ s_{i}(\widetilde{z}) s_{j}(\widetilde{z}) & , i \neq j,\end{cases}
$$

and

$$
\begin{aligned}
b_{i j}(\widetilde{z}): & =\left\langle\mathbf{N}(\widetilde{z}), \mathbf{s}_{i j}(\widetilde{z})\right\rangle \\
& =\left\langle \pm \frac{1}{\sqrt{1+\sum_{j=2}^{d} s_{j}(\widetilde{z})^{2}}} \cdot\left(\mathbf{e}_{\mathbf{1}}-\sum_{j=2}^{d} s_{j}(\widetilde{z}) \cdot \mathbf{e}_{j}\right), s_{i j}(\widetilde{z}) \cdot \mathbf{e}_{1}\right\rangle \\
& = \pm \frac{1}{\sqrt{1+\sum_{j=2}^{d} s_{j}(\widetilde{z})^{2}}} \cdot s_{i j}(\widetilde{z}) .
\end{aligned}
$$

So, up to the scaling factor, the matrix $\mathcal{B}(\widetilde{z})$ is equivalent to the Hessian of $s$ at the point $\widetilde{z}$. According to A.2.1, the principal curvatures of the hypersurface $\mathbf{s}$ at the point $\mathbf{s}(\widetilde{z})$ are given by the eigenvalues of the matrix $\mathcal{L}(\widetilde{z}):=\mathcal{G}(\widetilde{z})^{-1} \mathcal{B}(\widetilde{z})$. The eigenvectors of $\mathcal{L}(\widetilde{z})$ are the coordinate vectors of the corresponding principal curvature directions in the basis $\left\{\mathbf{s}_{2}(\widetilde{z}), \ldots, \mathbf{s}_{d}(\widetilde{z})\right\}$ of the tangent space of $\mathbf{s}$ at $\mathbf{s}(\widetilde{z})$. Hence, if $u=\left(u_{2}, \ldots, u_{d}\right) \in \mathbb{R}^{d-1}$ is an eigenvector of $\mathcal{L}(\widetilde{z})$, the corresponding principal curvature direction is

$$
\begin{aligned}
v & :=\sum_{j=2}^{d} u_{j} \cdot \mathbf{s}_{j}(\widetilde{z}) \\
& =\sum_{j=2}^{d} u_{j}\left(s_{j}(\widetilde{z}) \cdot \mathbf{e}_{1}+\mathbf{e}_{j}\right) \\
& =\left(\begin{array}{c}
u^{\top} \cdot \nabla s(\widetilde{z}) \\
u
\end{array}\right) .
\end{aligned}
$$

A very important special case is given by $\nabla s(\widetilde{z})=\mathbf{0}$. If $s_{2}(\widetilde{z})=\ldots=s_{d}(\widetilde{z})=0$ holds true, we have $\mathcal{G}(\widetilde{z})=\mathrm{I}_{d-1}, \sum_{j=2}^{d} s_{j}(\widetilde{z})^{2}=0$, and hence the matrix $\mathcal{L}(\widetilde{z})=\mathcal{B}(\widetilde{z})$ coincides - up to the sign - with the Hessian of $s$ at the point $\widetilde{z}$. Then, up to the sign, the principal curvatures are simply the eigenvalues of the Hessian of $s$ at the point $\widetilde{z}$. Furthermore, if $u \in \mathbb{R}^{d-1}$ is an eigenvector of $\mathcal{L}(\widetilde{z})=\mathcal{B}(\widetilde{z})$, the corresponding principal direction is

$$
v=\left(\begin{array}{l}
0 \\
u
\end{array}\right) \in \mathbb{R}^{d}
$$




\section{APPENDIX B}

\section{WEAK CONVERGENCE OF POINT PROCESSES}

\section{B.1 WEAK CONVERGENCE ON METRIC SPACES}

A basic tool used in this work is the weak convergence of point processes. This type of convergence has to be understood in the sense of weak convergence of random elements on metric spaces as studied in Billingsley [4, p. 7]: Given a complete and separable metric space $S$ with metric $\rho$ and Borel $\sigma$-field $\mathcal{S}$, a random element $X$ of $S$ is a measurable map from some probability space $(\Omega, \mathcal{A}, \mathbb{P})$ into $(S, \mathcal{S})$. A sequence $\left(X_{n}\right)_{n \geq 0}$ of random elements with corresponding distributions $\mathbb{P}_{n}:=\mathbb{P} \circ X_{n}^{-1}, n \geq 0$, converges weakly to $X_{0}$ if

$$
\int_{S} f(z) \mathbb{P}_{n}(\mathrm{~d} z) \rightarrow \int_{S} f(z) \mathbb{P}_{0}(\mathrm{~d} z)
$$

for each bounded and continuous function $f: S \rightarrow \mathbb{R}$, and we write $X_{n} \stackrel{\mathcal{D}}{\longrightarrow} X$ if (B.1) holds. Given the weak convergence on some metric space $S$, many powerful results can be used, for example the continuous mapping theorem.

Like in Section 2.2, let $D$ be a subset of a compactified Euclidean space of finite dimension and $\mathcal{D}$ the Borel $\sigma$-field of subsets of $D$. Furthermore, we again write $M_{p}(D)$ for the space of all counting measures $\chi$ of the form $\chi=\sum_{i=1}^{\infty} \varepsilon_{z_{i}}$, where $\left\{z_{i}, i \geq 1\right\}$ is a countable collection of not necessarily distinct points of $D$, that satisfies $\chi(K)<\infty$ for each compact set $K \in \mathcal{D}$. It would be desirable to be able to apply the theory of weak convergence on metric spaces to the space $S=M_{p}(D)$. 
For this purpose, it is necessary to show the existence of a metric $\rho$ on $M_{p}(D)$ which makes $\left(M_{p}(D), \rho\right)$ a complete and separable metric space. The next section will illustrate the most important steps to prove the existence of such a metric $\rho$.

\section{B.2 The Space $M_{p}(D)$ Is metrizable}

Like in Section 2.2, we call a measure $\mu$ on $D$ a Radon-measure if it takes finite values on each compact subset of $D$. In a first step, Resnick [21, p. 145] demonstrates that $M_{p}(D)$ is a closed subspace of

$$
M_{+}(D):=\{\mu: \mu \text { is a Radon-measure on } D\}
$$

with respect to a suitable topology, the so-called vague topology, see below for a formal definition. In a second step, he shows the existence of a metric $\rho$ which induces this topology and renders $\left(M_{+}(D), \rho\right)$ a complete and separable metric space, see Proposition 3.17 in Resnick [21]. Since each subspace of a separable metric space is separable (Willard [26, Problem 16G 1.]) and each closed subset of a complete metric space is complete (Willard [26, Theorem 24.10]), we can then conclude that $\left(M_{p}(D), \rho\right)$ itself is a complete and separable metric space. So, we can focus completely on the space $M_{+}(D)$ in the following.

Let $\mathcal{M}_{+}(D)$ be the smallest $\sigma$-field of subsets of $M_{+}(D)$, rendering the evaluation maps $\chi \mapsto \chi(A)$ from $M_{+}(D) \rightarrow[0, \infty]$ measurable for each $A \in \mathcal{D}$. A random measure is a measurable map from some probability space $(\Omega, \mathcal{A}, \mathbb{P})$ into $\left(M_{+}(D), \mathcal{M}_{+}(D)\right)$, and if its distribution is concentrated on $M_{p}(D)$, it is a point process. For showing that $M_{+}(D)$ is metrizable into a complete and separable metric space, it has to be endowed with the so-called vague topology on $M_{+}(D)$, generated by the mappings $\chi \rightarrow \int g \mathrm{~d} \chi, g \in C_{K}^{+}(D)$, where $C_{K}^{+}(D)$ is the set of all continuous functions from $D$ into $[0, \infty)$ with compact support, see Kallenberg [15, p. 316]. A basis of the vague topology is given by finite intersections of sets of the form

$$
\left\{\chi \in M_{+}(D): s<\int g \mathrm{~d} \chi<t\right\}
$$

for some $g \in C_{K}^{+}(D)$ and $s<t$, see Resnick [21, p. 140]. Now that we can speak of open sets in $M_{+}(D)$, it is natural to ask how the Borel $\sigma$-field $\mathcal{B}\left(M_{+}(D)\right)$, the $\sigma$-field generated by the open sets, is related to the $\sigma$-field $\mathcal{M}_{+}(D)$ we have seen before. We simply have

$$
\mathcal{B}\left(M_{+}(D)\right)=\mathcal{M}_{+}(D),
$$


see Jagers [12, p. 187]. According to Proposition 3.17 of Resnick [21], the space $M_{+}(D)$, equipped with the vague topology, is metrizable as a complete and separable metric space. For reasons of completeness, we sketch the construction of the corresponding metric $\rho$ : The basic idea is to find a countable subset $H$ of $C_{K}^{+}(D)$ so that we have

$$
\int g \mathrm{~d} \chi_{n} \rightarrow \int g \mathrm{~d} \chi_{0}, \quad \forall g \in C_{K}^{+}(D) \quad \Longleftrightarrow \quad \int h \mathrm{~d} \chi_{n} \rightarrow \int h \mathrm{~d} \chi_{0}, \quad \forall h \in H
$$

for any sequence $\left(\chi_{n}\right)_{n \geq 0}$ in $M_{+}(D)$. It is said that $\chi_{n}$ converges vaguely to $\chi_{0}$ if, and only if, the left-hand side of the equation above holds true. An explicit construction of such a countable set $H$ can be found in the proof of Proposition 3.17 of Resnick 21] or in Kallenberg [14, p. 170]. Writing $H=\left\{h_{1}, h_{2}, \ldots\right\}$, a suitable metric on $M_{+}(D)$ is given by

$$
\rho\left(\chi_{1}, \chi_{2}\right):=\sum_{i=1}^{\infty} \frac{1}{2^{i}}\left(1-\exp \left(-\left|\int h_{i} \mathrm{~d} \chi_{1}-\int h_{i} \mathrm{~d} \chi_{2}\right|\right)\right),
$$

$\chi_{1}, \chi_{2} \in M_{+}(D)$, see Resnick [21, p. 148].

The reasoning at the beginning of this section shows that $\left(M_{p}(D), \rho\right)$ is a complete and separable metric space, too, so that we can apply the theory of weak convergence on metric spaces to point processes.

\section{B.3 Results ON WEAK CONVERGENCE OF POINT PROCESSES}

To obtain a better understanding of the weak convergence of point processes, we state the following result, which is a special case of Theorem 16.16 in Kallenberg [15]. For this purpose, remember that we have called a point process $\xi$ simple if its distribution is concentrated on the simple point measures on $D$, i.e. if

$$
\mathbb{P}(\xi(\{z\}) \in\{0,1\} \text { for all } z \in D)=1 \text {. }
$$


Theorem B.1 (Special case of Theorem 16.16 in Kallenberg [15]). Let $\xi, \xi_{1}, \xi_{2}, \ldots$ be point processes on $D$. Then the following conditions are equivalent:

i) $\xi_{n} \stackrel{\mathcal{D}}{\longrightarrow} \xi$;

ii) $\int_{D} g \mathrm{~d} \xi_{n} \stackrel{\mathcal{D}}{\longrightarrow} \int_{D} g \mathrm{~d} \xi$ for each $g \in C_{K}^{+}(D)$;

iii) $\left(\xi_{n}\left(A_{1}\right), \ldots, \xi_{n}\left(A_{k}\right)\right) \stackrel{\mathcal{D}}{\longrightarrow}\left(\xi\left(A_{1}\right), \ldots, \xi\left(A_{k}\right)\right)$ for any choice of $k \in \mathbb{N}$ and relatively compact sets $A_{1}, \ldots, A_{k} \in \mathcal{D}$ with $\xi\left(\partial A_{i}\right)=0$ a.s. for $1 \leq i \leq k$.

If $\xi$ is a simple point process, it is also equivalent that

iv) $\xi_{n}(A) \stackrel{\mathcal{D}}{\longrightarrow} \xi(A)$ for each relatively compact set $A \in \mathcal{D}$ with $\xi(\partial A)=0$ a.s..

Since Proposition 3.22 stated in Resnick [21] has been a very essential part in the proof of Theorem 3.5, we recall it at this place:

Theorem B.2 (Proposition 3.22 of Resnick [21]). Suppose $\xi$ is a simple point process on $D$ and $\mathcal{I} \subset \mathcal{D}$ is a basis of relatively compact open sets, which is closed under finite unions and intersections and satisfies $\mathbb{P}(\xi(\partial I)=0)=1$ for each $I \in \mathcal{I}$. If $\xi_{n}, n \geq 1$, are point processes on $D$ and for all $I \in \mathcal{I}$

$$
\mathbb{P}\left(\xi_{n}(I)=0\right) \rightarrow \mathbb{P}(\xi(I)=0)
$$

and

$$
\mathbb{E}\left[\xi_{n}(I)\right] \rightarrow \mathbb{E}[\xi(I)]<\infty
$$

then

$$
\xi_{n} \stackrel{\mathcal{D}}{\longrightarrow} \xi
$$

in $M_{p}(D)$.

The last result we want to recapitulate is a so-called de-Poissonization result stated in Mayer and Molchanov [20]. This result is the key argument why it was sufficient to investigate $\operatorname{diam}\left(\mathbf{Z}_{n}\right)$ instead of $M_{n}$. For some more details, see Chapter 3 of Mayer and Molchanov [20].

Theorem B.3 (Special case of Theorem 3.2 of Mayer and Molchanov [20]). Let $\Psi: M_{p}\left(\mathbb{R}^{d}\right) \rightarrow \mathbb{R}$ be a non-increasing functional. Furthermore, let $\Pi_{n \kappa}$ be a Poisson process with intensity measure $n \kappa$, where $\kappa$ is a probability measure on $\mathcal{B}^{d}$ and $a_{n}=c n^{\alpha}, n \in \mathbb{N}$ with $c, \alpha>0$. If the random variable $a_{n} \Psi\left(\Pi_{n \kappa}\right)$ converges in distribution to a random variable with cumulative distribution function $F$, then the distribution of $a_{n} \Psi\left(\Xi_{n}\right)$ also converges weakly to $F$, where $\Xi_{n}$ is a binomial process of $n$ i.i.d. points with common distribution $\kappa$. 
In the proof of Theorem 3.5 we applied this result to the functional $\Psi(\chi)=$ $2 a-\operatorname{diam}(\chi)$, the processes $\Pi_{n \kappa}=\mathbf{Z}_{n}$, i.e. $\kappa=\mathbb{P}_{Z}$, see Section $2.2, \Xi_{n}=\sum_{j=1}^{n} \varepsilon_{Z_{i}}$ and $a_{n}=n^{2 /(d+1)}$. To this end, observe that

$$
\Psi\left(\Xi_{n}\right)=2 a-\operatorname{diam}\left(\sum_{j=1}^{n} \varepsilon_{Z_{i}}\right)=2 a-\max _{1 \leq i, j \leq n}\left|Z_{i}-Z_{j}\right|=2 a-M_{n} .
$$





\section{BIBLIOGRAPHY}

[1] Abramowitz, M. and I. A. Stegun (1964). Handbook of Mathematical Functions: with Formulas, Graphs, and Mathematical Tables, Volume 55 of Applied Mathematics Series. National Bureau of Standards.

[2] Aminov, Y. (2001). The Geometry of Submanifolds. Amsterdam: Gordon and Breach Science Publishers.

[3] Appel, M. J., C. A. Najim, and R. P. Russo (2002). Limit laws for the diameter of a random point set. Advances in Applied Probability 34(1), 1-10.

[4] Billingsley, P. (1999). Convergence of Probability Measures (second ed.). Wiley Series in Probability and Statistics. New York: Wiley.

[5] Csikós, B. (2014). Differential Geometry. Series of Lecture Notes and Workbooks for Teaching Undergraduate Mathematics. Budapest: Typotex Publishing House.

[6] Daley, D. J. and D. Vere-Jones (2008). An Introduction to the Theory of Point Processes, Volume 2: General Theory and Structure (second ed.). New York: Springer.

[7] Demichel, Y., A.-K. Fermin, and P. Soulier (2015). The diameter of an elliptical cloud. Electronic Journal of Probability 20(27), 1-32.

[8] Fang, K.-T., S. Kotz, and K.-W. Ng (1990). Symmetric Multivariate and Related Distributions, Volume 36 of Monographs on Statistics and Applied Probability. Springer.

[9] Gray, A. (1993). Modern Differential Geometry of Curves and Surfaces. Studies in Advanced Mathematics. London: CRC Press. 
[10] Henze, N. and T. Klein (1996). The limit distribution of the largest interpoint distance from a symmetric Kotz sample. Journal of Multivariate Analysis 57(2), 228-239.

[11] Henze, N. and W. Lao (2010). The limit distribution of the largest interpoint distance for power-tailed spherically decomposable distributions and their affine images. Preprint.

[12] Jagers, P. (1974). Aspects of random measures and point processes. In P. Ney and S. Port (Eds.), Advances in Probability and Related Topics, Volume 3. New York: Marcel Dekker.

[13] Jammalamadaka, S. R. and S. Janson (2015). Asymptotic distribution of the maximum interpoint distance in a sample of random vectors with a spherically symmetric distribution. The Annals of Applied Probability 25(6), 3571-3591.

[14] Kallenberg, O. (1983). Random Measures (third ed.). Berlin: Akademie.

[15] Kallenberg, O. (2002). Foundations of Modern Probability (second ed.). New York: Springer.

[16] Lao, W. (2010). Some Weak Limit Laws for the Diameter of Random Point Sets in Bounded Regions. Karlsruhe: KIT Scientific Publishing.

[17] Last, G. and M. Penrose. Lectures on the Poisson Process. To be published as IMS Textbook. Cambridge University Press. Preliminary draft from 23 February 2017, available at http://www.math.kit.edu/stoch/ last/seite/lectures_on_the_poisson_process/de.

[18] Lee, J. M. (1997). Riemannian Manifolds: An Introduction to Curvature, Volume 176 of Graduate Texts in Mathematics. New York: Springer.

[19] Matthews, P. C. and A. L. Rukhin (1993). Asymptotic distribution of the normal sample range. The Annals of Applied Probability 3(2), 454-466.

[20] Mayer, M. and I. Molchanov (2007). Limit theorems for the diameter of a random sample in the unit ball. Extremes 10(3), 129-150.

[21] Resnick, S. I. (2008). Extreme Values, Regular Variation, and Point Processes. Springer Series in Operations Research and Financial Engineering. New York: Springer. 
[22] Schneider, R. (2014). Convex Bodies: The Brunn-Minkowski Theory (second ed.), Volume 151 of Encyclopedia of Mathematics and its Applications. Cambridge University Press.

[23] Schrempp, M. (2015). The limit distribution of the largest interpoint distance for distributions supported by an ellipse and generalizations. arXiv preprint arXiv:1505.01597.

[24] Schrempp, M. (2016). The limit distribution of the largest interpoint distance for distributions supported by a $d$-dimensional ellipsoid and generalizations. Advances in Applied Probability 48(4), 1256-1270.

[25] Wang, X. (2005). Volumes of generalized unit balls. Mathematics Magazine 78(5), 390-395.

[26] Willard, S. (1970). General Topology. Addison-Wesley Series in Mathematics. London: Addison-Wesley Publishing Company.

[27] Williams, D. (1991). Probability with Martingales. Cambridge Mathematical Textbooks. Cambridge University Press. 